Ph. D. Dissertation

\title{
SYSTEMATIC DESIGN OF ANTENNAS USING THE THEORY OF CHARACTERISTIC MODES
}

Author: Marta Cabedo Fabrés

Advisor: Alejandro Valero Nogueira

February 2007 

To my parents.

To José Manuel. 



\section{Acknowledgements}

I would like to express my sincere gratitude and appreciation to my advisor Alejandro Valero for his expert guidance, patience, and keen interest throughout the course of this work. He has taught me to do my research in an organized and meticulous way.

I am also grateful to Miguel Ferrando not only for his help and valuable suggestions for this thesis, but also for his advice and support in my professional career.

I am especially indebted to my faithful colleague Eva Antonino, for her friendship, support and encouragement. Without her help this work would have been a lot harder.

I would like to thank José Ignacio Herranz for sharing with me his optimism and intelligence. He is always willing to help anyone.

Acknowledgements are also due to Mariano Baquero and Vicent Miquel Rodrigo, and to the fellow companions at GRE laboratory, Bernat, Esperanza, Daniel, David, Carlos, Fulvio, and Felipe, for their support and friendship.

My parents and my family stood behind me for which I am thankful for them forever.

Finally, to my beloved husband José Manuel go my most loving thanks for his patience, understanding and unlimited encouragement. 


\section{Abstract}

The main goal of this thesis is to show how the Theory of Characteristic Modes can be systematically applied to design wire and planar antennas. Through numerous examples, it will be demonstrated that in contrast with other classical design methods, the Theory of Characteristic Modes brings insight into the physical phenomena taking place on an antenna.

Examples will be presented in order to demonstrate that an in depth knowledge of the radiating mechanisms of very basic antennas helps to design novel antennas on a clear and rational basis. It will be also explained how the information given by characteristic modes can be used for the selection of the most suitable shape for the radiating element, as well as for the choice of an optimum feeding arrangement to maximize the impedance bandwidth.

The Theory of Characteristic Modes was first formulated by Garbacz in 1968, and later refined by Harrington and Mautz in 1971. Traditionally, characteristic modes have been applied to antenna shape synthesis, and control of obstacle scattering by reactive loading. However, at present, the Theory of Characteristic Modes has practically fallen into disuse, in spite of the fact that it leads to modal solutions, which are particularly useful in problems involving analysis, synthesis and optimization of antennas and scatterers.

Characteristic modes are real current modes that correspond with the eigenvectors of a particular weighted eigenvalue equation that involves the generalized impedance matrix of the body. Thus, characteristic modes can be computed numerically for conducting bodies of arbitrary shape, and since they form a set of orthogonal functions, they can be used to expand the total current on the surface of the body. However, what makes characteristic modes really attractive for antenna design is the physical insight they bring into the radiating phenomena taking place in the antenna.

Associated to each characteristic mode there is an eigenvalue whose magnitude provides information about the resonant frequency and radiating characteristics of modes. Additionally, since characteristic modes are computed in the absence of any kind of excitation, they only depend on the shape and size of the conducting object. Hence, antenna design using characteristic modes can be performed in a controlled way following two steps:

- Firstly, the shape and size of the radiating element are optimized on the base of the information provided by eigenvalues.

- Next, studying the current distribution of modes an optimum feeding arrangement is chosen so that the desired mode or modes may be excited, in order to obtain a specific radiating behaviour. 


\section{Resumen}

El principal objetivo de esta tesis es demostrar que la Teoría de los Modos Característicos puede ser empleada de forma sistemática para diseñar antenas de hilo y antena planas. La gran ventaja de los modos característicos, frente a otros métodos de diseño, es la clara visión física que proporcionan de los fenómenos que contribuyen a la radiación de la antena.

A través de numerosos ejemplos se demostrará como los modos característicos permiten comprender mejor el funcionamiento de una antena, de forma que el diseño de la misma se puede realizar de forma justificada y coherente. También se mostrará como la información proporcionada por los modos característicos puede ser aprovechada para seleccionar la forma más apropiada para el elemento radiante, al igual que para elegir una configuración de alimentación óptima que maximice el ancho de banda de impedancia.

La Teoría de los Modos Característicos fue inicialmente formulada por Garbacz en 1968, y posteriormente refinada por Harrington y Mautz en 1971. Tradicionalmente, los modos característicos han sido empleados para sintetizar formas de antena, y para controlar la difracción de objetos mediante carga reactiva. Sin embargo, en la actualidad, la Teoría de los Modos Característicos ha caído prácticamente en el olvido, a pesar de que permite obtener una solución modal para la corriente, que es de gran utilidad a la hora de analizar problemas de análisis, síntesis y optimización de antenas y difractores.

La Teoría de los Modos Característicos parte de la definición de un problema de autovalores que involucra la matriz de impedancia generalizada de la estructura, y que tras ser resuelto proporciona un conjunto de modos de corriente reales, que son los denominados modos característicos. Estos modos se corresponden con las resonancias naturales de la estructura y pueden ser obtenidos numéricamente para cuerpos conductores de forma arbitraria.

Por otra parte, los modos característicos forman un conjunto de funciones cerrado y ortogonal, por lo que pueden ser empleados para expandir la corriente superficial que fluye por el cuerpo conductor. Sin embargo, lo que hace a los modos característicos especialmente atractivos para el diseño de antenas es la visión física que aportan de los fenómenos de radiación que determinan el comportamiento de la antena.

Asociado a cada modo característicos existe un autovalor, cuya magnitud proporciona información sobre la frecuencia de resonancia y las propiedades de radiación de los modos. Además, puesto que los modos característicos se calculan en ausencia de cualquier excitación, únicamente dependen de la forma y del tamaño del cuerpo conductor. Por tanto, el diseño de la antena se puede llevar a cabo de forma controlada en dos pasos: 
- En primer lugar, se optimiza la forma y el tamaño del elemento radiante en base a la información proporcionada por los autovalores.

- A continuación, a partir del estudio de la distribución de corriente de los modos se determina una configuración de alimentación óptima que permita excitar el modo o modos deseados, con el fin de obtener un comportamiento radiante determinado. 


\section{Resum}

El principal objectiu d'aquesta tesi és demostrar que la Teoria dels Modes Característics pot ser emprada de forma sistemàtica per a disenyar antenes de fil i antenes planes. El gran avantatge dels modes característics, enfront d'altres mètodes de disseny, és la clara visió física que proporcionen dels fenòmens que contribuïxen a la radiació de l'antena.

Mitjançant nombrosos exemples es demostrarà com els modes característics permeten comprendre millor el funcionament d'una antena, de manera que el disseny de la mateixa es pot realitzar de forma justificada i coherent. També es mostrarà com la informació proporcionada pels modes característics pot ser aprofitada per a seleccionar la forma més apropiada per a l'element radiant, igual que per a triar una configuració d'alimentació òptima que maximitze l'ample de banda d'impedància.

La Teoria dels Modes Característics va ser inicialment formulada per Garbacz en 1968, i posteriorment refinada per Harrington i Mautz en 1971. Tradicionalment, els modes característics han sigut emprats per a sintetitzar formes d'antena, i per a controlar la difracció d'objectes per mitjà de càrrega reactiva. No obstant, en l'actualitat, la Teoria dels Modes Característics ha caigut pràcticament en l'oblit, a pesar que permet obtindre una solució modal per al corrent, que és de gran utilitat a l'hora d'analitzar problemes d'anàlisi, síntesi i optimització d'antenes i difractors.

La Teoria dels Modes Característics partix de la definició d'un problema d'autovalors que involucra la matriu d'impedància generalitzada de l'estructura, i que després de ser resolt proporciona un conjunt de modes de corrent real, que són els denominats modes característics. Estos modes es corresponen amb les ressonàncies naturals de l'estructura i poden ser obtinguts numèricament per a cossos conductors de forma arbitrària.

D'altra banda, els modes característics formen un conjunt de funcions tancat i ortogonal, per la qual cosa poden ser emprats per a expandir el corrent superficial que fluïx pel cos conductor. No obstant, allò que fa als modes característics especialment atractius per al disseny d'antenes és la visió física que aporten dels fenòmens de radiació que determinen el comportament de l'antena.

Associat a cada mode característics hi ha un autovalor, la magnitud del qual proporciona informació sobre la freqüència de ressonància $i$ les propietats de radiació dels modes. A més, ja que els modes característics es calculen en absència de qualsevol excitació, únicament depenen de la forma i de la grandària del cos conductor. Per tant, el disseny de l'antena es pot dur a terme de forma controlada en dos passos: 
- $\quad$ En primer lloc, s'optimitza la forma i la grandària de l'element radiant basant-se en la informació proporcionada pels autovalors.

- A continuació, a partir de l'estudi de la distribució de corrent dels modes es determina una configuració d'alimentació òptima que permeta excitar el mode o modes desitjats, a fi d'obtindre un comportament radiant determinat. 


\section{CONTENTS}

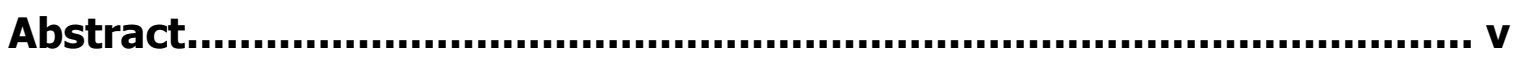

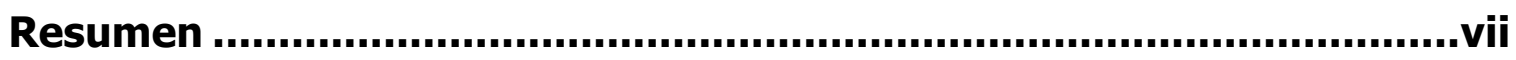

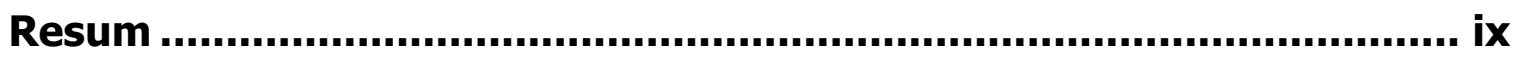

Chapter 1. Introduction and Overview ........................................... 3

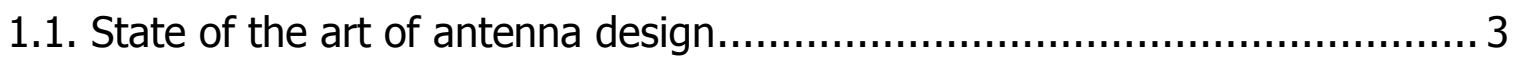

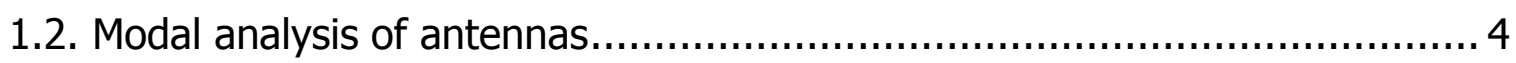

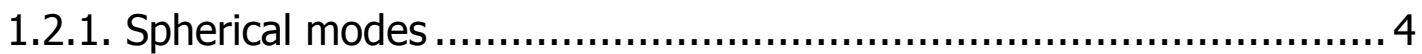

1.2.2. Modal expansion methods ..................................................... 5

1.2.3. Eigenfunctions of conducting bodies........................................... 5

1.3. Application of the Theory of Characteristic Modes to antenna design .......... 6

1.4. General objective of the thesis.......................................................... 8

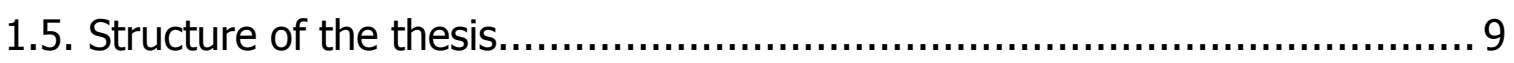

Chapter 2. The Theory of Characteristic Modes Revisited.................. 11

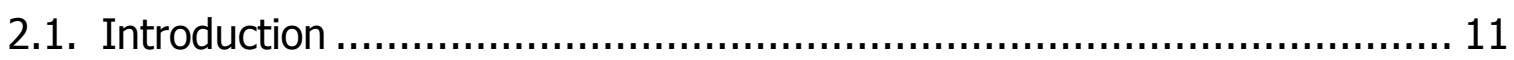

2.2. Mathematical formulation of Characteristic Modes ............................... 12

2.2.1. Characteristic currents ........................................................ 13

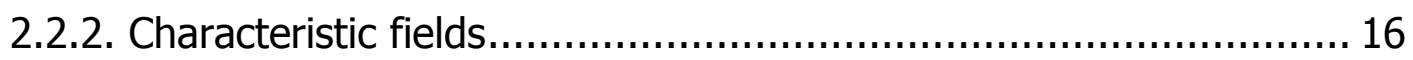

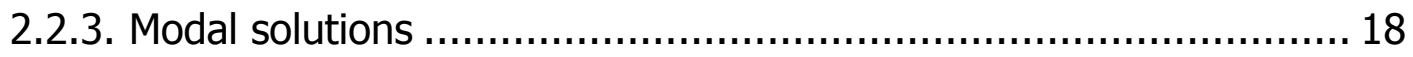

2.3. Physical interpretation of Characteristic Modes ................................... 19

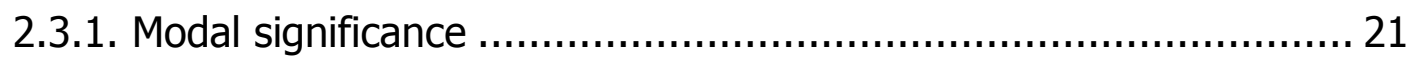

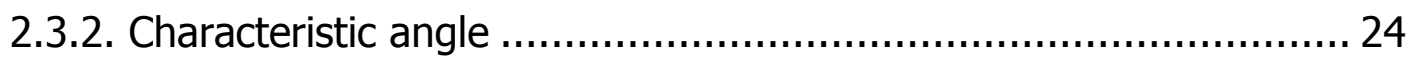

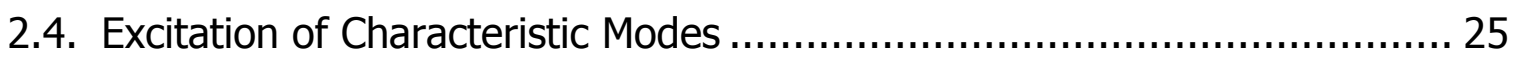

2.5. Mode resonance by reactive loading ............................................... 27

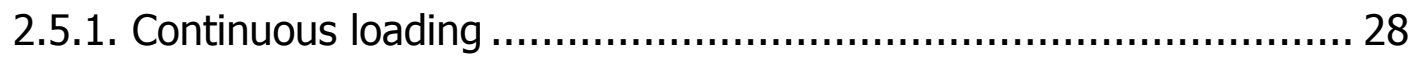

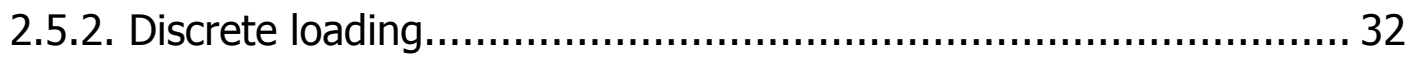

2.6. Equivalent circuit of an antenna using Characteristic Modes ................... 34 
Chapter 3. Modal Analysis of Wire Loop Antennas............................. 37

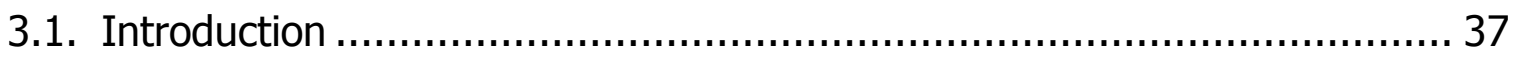

3.2. Special non-resonant modes and degenerated modes........................... 37

3.3. Use of reactive loading for obtaining circular polarization ....................... 44

3.4. Effect of the symmetry of loops on the degeneracy of modes .................. 47

3.4.1. Modal analysis of the square loop antenna.................................. 47

3.4.2. Modal analysis of a regular triangular loop antenna...................... 55

3.5. Antenna modes and transmission line modes ......................................6 60

3.6. Modal characterization of complex wire structures............................... 64

Chapter 4. On the Convergence of Characteristic Modes..................... 69

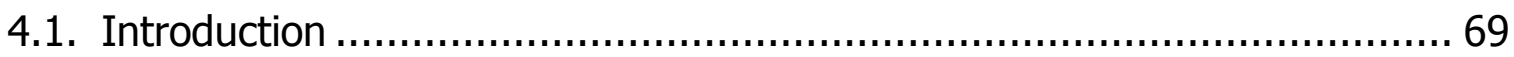

4.2. The use of characteristic modes as entire-domain basis functions for large

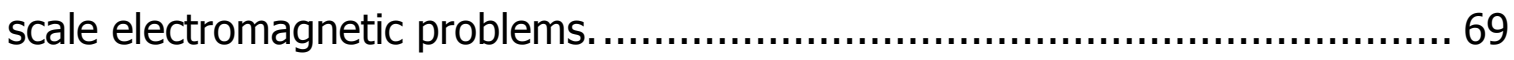

4.3. Slow convergence of the series of characteristic modes for wire antennas. 71

4.4. The source mode for wire antennas. ............................................... 74

4.5. Frequency independent basis functions............................................. 80

4.6. Convergence of the series of characteristic modes for patch antennas...... 86

4.7. Convergence of the series of characteristic modes for coaxial fed patches. 95

Chapter 5. Modal Analysis of Planar Antennas.................................. 97

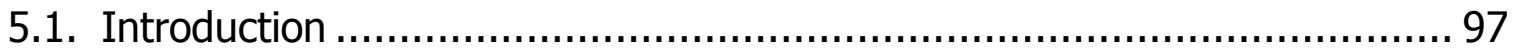

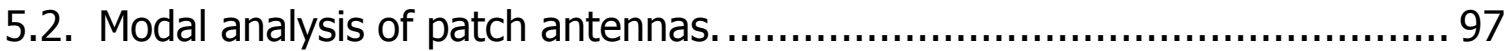

5.2.1. Computation of characteristic modes for arbitrary shaped plates. .. 98

5.2.2. Determination of the optimum height of a microstrip patch above a ground plane to obtained maximum radiating bandwidth. 105

5.2.3. Generation of circular polarization in arbitrarily shaped patch antennas

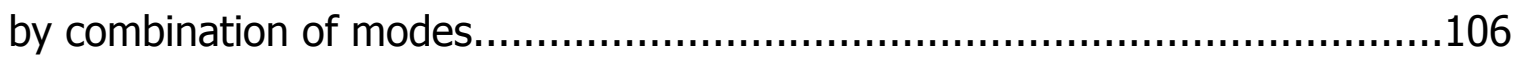

5.2.4. Reduction of the cross polarization level for reflectarray antennas. 107

5.2.5. Coupling between planar structures....................................... 113

5.2.5.1. Coupling phenomena in coplanar plates ........................... 113

5.2.5.2. Coupling phenomena in parallel plates..............................118 
5.3. Modal analysis of planar monopoles

.121

5.3.1. Influence of the shape of planar monopoles in the impedance bandwidth performance. .122

5.3.2. Modal analysis of a non-centred fed monopole.

5.3.3. Effect of the addition of a shorting pin on the monopole base. 139

5.3.4. Use of a double feeding configuration to improve the input bandwidth of planar monopoles.

5.3.5. Application of planar monopole antennas to MIMO systems. .152

Chapter 6. Modal Analysis of Radiating Ground Planes 155

6.1. Introduction 155

6.2. Design of a folded radiating ground plane antenna for mobile terminals 156

6.2.1. Modal analysis of the basic folded radiating ground plane. 157

6.2.2. Determination of the optimum feeding arrangement................ 159

6.2.3. Effect of the insertion of a slot. 163

6.2.4. Use of a bow-tie shaped feeding monopole. 168

6.2.5. Conclusions 169

6.3. Design of notched radiating ground planes. 170

6.3.1. Radiating ground plane with a notch 170

6.3.2. Radiating ground plane with two notches 174

Chapter 7. Conclusions and Future Work 179

7.1. Conclusions 179

7.1.1. Suitability of characteristic modes for antenna design. 179

7.1.2. Suitability of characteristic modes for antenna design. 181

7.2. Future Work 182

References. 183

Appendix 1. Computation of eigenvectors and eigenvalues. 191

Appendix 2. The Mixed Potential Integral Equation. 195

Related Publications. 205 




\section{CHAPTER 1. Introduction and Overview}

\subsection{State of the art of antenna design}

In past years the rise of wireless communications has gathered significant interest in antenna design. In particular, due to market demand, design of small antennas for new mobile terminals [1]-[4] is currently receiving a lot of attention. Nevertheless, designing a handset antenna is not an easy task, as this type of antenna is subjected to very stringent specifications [5]. Small size, light weight, compact structure, low profile, robustness and flexibility are the prime considerations conventionally taken into account in small antenna design [6]. In addition, as new mobile handsets are required to operate at multiple standards, their antennas are expected to grab as much spectrum as possible, so they may provide multi-band or broadband operation [7].

Unfortunately, as the antenna geometry complicates, more often than not, there is no close formulation to analyze it, and the use of numerical methods [8]-[10] becomes imperative. In consequence, design of modern antennas relies on the use of self-developed numerical codes or commercial electromagnetic simulators, such as IE3D [11], FEKO [12], Empire [13], or HFSS [14], among others, to evaluate antenna performance before a physical prototype is fabricated. Under this circumstance, time for antenna design can be dramatically reduced thanks to computers. Anyway, even with the support of computers, the success of the final design depends upon the intuition and previous experience of the designer, and in most cases, the final optimization is in fact made by "cut and try" methods. As a result, these days antenna design is very much governed by the designer expertise and know-how.

On the other hand, an alternative and certainly in vogue approach to design handset antennas consists of using automated optimization techniques based on pseudo-random search algorithms [15]. Typical examples of these techniques are genetic algorithms [16]-[17], artificial neural networks [18]-[19], particle swarm optimization [20] or bees algorithms [21]. Their main advantage is that once the optimization algorithm is programmed, little interaction with the designer is required, as the computer is supposed to arrive at the expected specifications autonomously.

As a matter of fact, although all the above mentioned design strategies are really suitable when time-to-market is critical, their major problem is that they are rather lacking in physical insight, so real knowledge of the operating principles of the antenna is mislaid. Actually, publications giving useful instructions for better antenna design are scarce. Nevertheless, there exist other not so common design strategies, especially those based on modal analysis, which can alleviate this problem. 


\subsection{Modal analysis of antennas}

As explained before, at present antenna design is essentially focused on commercial applications, especially those related with cellular telephony and wireless systems. As a result, the main objective of antenna designers is obtaining antennas with the desired radiation characteristics in the shortest period of time, using the most effective design procedures. Because of this trend, commercial electromagnetic simulators and automated design techniques are gaining ground among antenna designers. Consequently, publications proposing new antennas are mainly devoted to describe the antenna geometry and its radiating behaviour, while the design procedure does not receive the consideration it deserves, and few attention is paid to the physics of the problem.

This thesis is aimed at giving a physical explanation to the radiation behaviour of different types of antennas. To some extent, modal analysis can cope with this objective, as it brings information about the modes that contribute to the radiation of the antenna for each particular excitation.

Modal analysis has long been used in electromagnetism for the analysis of close structures like waveguides and cavities, in which it is relatively easy to arrive at close solutions, even for structures of non-separable geometry, by applying boundary conditions [22]-[23]. However, the calculation of modes in open radiating structures, such as antennas or scatterers, is more involved, and it is usually quite time consuming. Probably, this is one of the reasons why modal analysis is not widely used for antenna design nowadays. In fact, most research dealing with the computation of modes in open structures was published more than forty years ago.

\subsubsection{Spherical modes}

A revision of the available literature regarding modal analysis shows that the first modal approach to antennas was taken by Chu in 1948 [24], and subsequently by Harrington in 1960 [25], when trying to determine the fundamental limitations in antennas. They postulated that, since any radiating field can be written as a sum of spherical vector waves, the antenna can be enclosed in a sphere. Then, the radiated power of the antenna is calculated from the propagating modes within the sphere, while all modes contribute to reactive power.

When the sphere enclosing the antenna is very small, there are no propagating modes, as all modes are evanescent (below cut-off). Unlike in a close waveguide, evanescent modes present a real part, so they provide a little real power. If there is only one propagating mode, the radiated power will arise primarily from that mode. However, if the sphere containing the antenna is large enough, several propagating modes will be supported, and the approach proposed by Chu [24] will be of little value as the modal coefficients will be difficult to calculate. 


\subsubsection{Modal expansion methods}

In the seventies, other more general modal expansion methods were developed [26]-[27]. These methods are based on expanding the fields radiated by an antenna in terms of planar, cylindrical or spherical wave functions. Next, the coefficients in the expansion are determined by measuring the near fields of the antenna.

When applying modal expansion methods, first the entire domain of interest should be divided into a number of subregions. Then, field expressions in all these subregions are expanded into the summation of their modal functions weighted by unknown coefficients. By enforcing the continuity conditions of tangential field components across the regional interfaces, one can then determine these expansion coefficients. Based on the obtained field expressions, the current distribution along the surface of the antenna can be readily calculated. Finally, the input impedance and radiation pattern of the antenna can be computed from the known current distribution.

\subsubsection{Eigenfunctions of conducting bodies}

In addition to the modal methods previously described, the eigenfunctions of classical analysis also constitute a modal approach to antenna problems. These eigenfunctions provide valuable solutions and physical insight for problems whose boundaries coincide with the constant coordinate surfaces of a particular coordinate system.

One type of generalization of the classical eigenfunctions, termed characteristic modes, was formulated by Garbacz in 1968 [28]. Characteristic modes defined by Garbacz correspond with the eigenvectors of a weighted eigenvalue equation. These modes have the useful property of being orthogonal over both the source region and the sphere at infinity [28]-[29].

In 1971, Harrington and Mautz obtained the same modes defined by Garbacz by diagonalizing the operator relating the current to the tangential electric field on the body [30]-[31]. The advantage of the formulation described in [30], known as the Theory of Characteristic Modes, is that it leads to a simpler derivation of the theory than the one proposed by Garbacz in [28], while it is valid for conducting bodies of arbitrary shape. Afterwards, Harrington et al. extended the formulation of the Theory of Characteristic Modes to encompass dielectric, magnetic, and both dielectric and magnetic bodies [32].

Some years later, in 1978, another modal formalism was introduced by Inagaki for problems of radiation and scattering from arbitrary discrete and continuous structures [33]. These modes, that are usually called Inagaki modes, correspond with the eigenfunctions of composite hermitian operators [34]. 
Inagaki modes can be considered more general than characteristic modes since they can be forced to be orthogonal over any sector of space, as well as orthogonal over the source region itself. Because of this characteristic, Inagaki modes have been applied to pattern synthesis and array optimization [35].

Most recently, in 1990 a modified version of Inagaki modes was proposed [36]. This new modes referred to as Generalized Characteristic Modes, are a generalization of Inagaki modes, providing advantages such as versatility and computational efficiency.

Among all the modal approaches alluded to above, the Theory of Characteristic Modes formulated in [30] is the one chosen to be analyzed in the present work. The choice has been based on the mathematical properties of characteristic modes, and on the physical insight they bring to the radiating phenomena taking place on the antenna.

As will be explained in next section, the use of characteristic modes can aid the construction of design procedures on a clear and rational basis.

\subsection{Application of the Theory of Characteristic Modes to antenna design}

Characteristic modes are real current modes that can be computed numerically for conducting bodies of arbitrary shape. Since characteristic modes form a set of orthogonal functions, they can be used to expand the total current on the surface of the body [30].

Traditionally, characteristic modes have been applied to antenna shape synthesis [37], and control of obstacle scattering by reactive loading [38]. However, at present, the Theory of Characteristic Modes has practically fallen into disuse, in spite of the fact that it leads to modal solutions, which are particularly useful in problems involving analysis, synthesis and optimization of antennas and scatterers [39]-[41].

Probably, the Theory of Characteristic Modes was almost abandoned in the eighties, because of the amount of computation it required for the extraction of modes. Characteristic modes correspond with the eigenvectors of a particular weighted eigenvalue equation that involves the generalized impedance matrix of the body. Hence, if characteristic modes are to be obtained, the impedance matrix of the body should be calculated at every desired frequency by direct application of Method of Moments [42].

Although Method of Moments calculations were a serious problem twenty years ago, at this time, they can be easily managed thanks to the huge computation capabilities of modern computers, and to the efficient matrix solvers developed for programming environments like Matlab [43]. 
Evidently, the current distribution on the surface of a conducting body can be computed in a more efficient and accurate way by direct inversion of the generalized impedance matrix. Nevertheless, for this work the use of characteristic modes as basis functions in which to expand the total current is preferred, as it lends more insight to the physical phenomena taking place on the antenna.

The main purpose of this thesis is to demonstrate that characteristic modes can be effectively used to carry out a controlled design of different types of antennas, because of the physical insight they provide.

Since characteristic modes are computed in the absence of any kind of excitation, they only depend on the shape and size of the conducting object. Thus, antenna design using characteristic modes can be performed in a controlled way following next procedure

- Firstly, characteristic currents and associated characteristic fields of the radiating element are calculated.

- Next, the resonance frequency of these modes, as well as their radiating behaviour, is determined from the information provided by the corresponding eigenvalues.

- Then, the shape and size of radiating element is modified until the desired resonant frequency or radiating characteristic is accomplished.

- Finally, studying the current distribution of modes an optimum feeding arrangement is chosen so that the desired mode or modes may be excited, in order to obtain a specific radiating behaviour.

For modelling electrically small conducting bodies few modes are required. Thus, small and intermediate size antennas can be fully characterized in a wide operating band just considering five or six characteristic modes.

In next chapters, characteristic modes are going to be used to perform a systematic analysis and design of different types of antennas which range from the wire antenna class, to planar antenna geometries, such as planar monopoles or patches.

Characteristic modes of $2 \mathrm{D}$ and $3 \mathrm{D}$ metallic structures are going to be computed using a code based on Mixed Potential Integral Equation (MPIE) [42] and Rao-Wilton-Glisson (RWG) functions [44]. This code, which has been programmed with Matlab, has been expressly developed to compute characteristic modes efficiently at a wide frequency band. The choice of Matlab as programming environment has been based on its simplicity, and on the robust matrix solvers it provides. Moreover, Matlab graphical interface facilitates the visualization of the current distribution of the modes for 2D and 3D structures [45]. Finally, execution times have been reduced compiling some parts of the code with Matlab $\mathrm{C}++$ compiler, and employing the interpolation procedure described in [46]. 


\subsection{General objective of the thesis}

As commented above, the main objective of this thesis is to recover the Theory of Characteristic Modes for antenna design applications.

In the development of this thesis, it will be shown that the Theory of Characteristic Modes is really helpful for antenna design as it provides a clear vision of the operating principles of the antenna.

Obviously, from an in-depth knowledge of the radiation mechanisms of the antenna, designs can be performed in a more coherent way. In fact, using the information provided by characteristic modes it is possible to establish a systematic design procedure, valid for different types of antennas.

Some of the issues that are going to be addressed in this work in order to show the suitability of characteristic modes for antenna design are the following:

- Analysis of the effect of loading wire antennas.

- Determination of a modal quality factor, and an equivalent circuit for wire antennas using the information provided by characteristic modes.

- Study of the degeneracy of modes for polygonal loop antennas.

- Acceleration of the convergence of the series of modes for the wire antenna class and for planar antennas.

- Determination of the optimum height of a patch above an infinite ground plane in order to obtain maximum bandwidth.

- Generation of circular polarization in patch antennas by combination of modes.

- Reduction of the cross polarization level for reflectarray antennas.

- Modelling of the coupling between open resonators.

- Analysis of the influence of the shape of planar monopoles in the impedance bandwidth performance.

- Use of a double feeding configuration in order to improve the input bandwidth of planar monopoles.

- Application of planar monopole antenna for MIMO systems.

- Determination of chassis-antenna modes in cellular handsets.

- Analysis of the radiation of a folded radiating ground plane.

- Notch antenna design on finite ground planes. 


\subsection{Structure of the thesis}

The first chapters of this thesis are devoted to the modal analysis of different wire antennas. Since the visualization of the current distribution of modes is easier in wire structures than in planar ones, wire antennas constitute the better option to initiate the reader into the Theory of Characteristic Modes.

In Chapter 2, the mathematical formulation of the Theory of Characteristic Modes will be reviewed, using as an example a straight wire dipole. The objective of this chapter is not really to study the wire dipole itself, which is a very well-known antenna, but to show all the possibilities yielded by characteristic modes for antenna design. Basically, the choice of the wire dipole as example has been based on its simplicity, which aids to understand the fundamentals of the modal theory.

Chapter 3 will be dedicated to the study of the characteristic modes of wire loops of different geometry. It will be demonstrated that in wire loops there are special non-resonant modes that present inductive behaviour at all frequencies. These special modes are characterized by currents forming close loops. A modal expansion for the input admittance of the antenna will be employed to determine which modes are excited for each particular feeding configuration. Additionally, it will be explained that drawing an analogy with the folded dipole, modes in wire loops can be classified in antenna or transmission-line modes, according to its currents flowing in phase or in opposite phase.

Chapter 4 will be focused on the analysis of the main drawback of characteristic modes, its slow converge when they are used to expand the total current on an antenna. Several examples will be presented in order to demonstrate that when an antenna is fed with a delta gap source, characteristic modes lead to ill convergent solutions for the imaginary part of the input current. This convergence problem will be overcome for both wire and planar antennas by adding to the series of characteristic modes an extra mode, with evanescent behaviour, called "source mode". Moreover, a procedure based on a Singular Value Decomposition (SVD) will be proposed in order to obtain a reduce set of Frequency Independent Characteristic Modes (FICM), appropriate to expand the total current very accurately, in a wide range of frequencies.

In Chapter 5, characteristic modes will be obtained for different planar antennas, such as patches and planar monopoles. Examples will be provided to show how the information given by characteristic modes can be used for the selection of the most suitable shape for the radiating element, as well as for the choice of an optimum feeding arrangement to maximize the impedance bandwidth. 
The aim of Chapter 6 will be to demonstrate that characteristic modes are very helpful for the design of handset antennas based on the resonance of the Printed Circuit Board (PCB) of the mobile terminal. The first part of the chapter will be devoted to the study of radiating structures that consist in a folded radiating ground plane. In the second part of the chapter, the behaviour of notched radiating ground planes will be investigated from a modal point of view.

Finally, Chapter 7 will summarize the most important conclusions of this thesis, and will include proposals for future work. 


\subsection{Introduction}

The Theory of Characteristic Modes was first developed by Garbacz in 1968 [28], and later refined by Harrington and Mautz in 1971 [30]. Garbacz obtained the modes of conducting bodies of arbitrary shape by diagonalizing the scattering matrix [29]. Alternatively, Harrington and Mautz arrived at the same modes by diagonalizing the generalized impedance matrix of the body [30]-[31]. This second approach is the one taken for this thesis, as it provides a more direct derivation of the theory and leads to explicit formulas for determining modal currents and fields.

Modal currents or characteristic currents are defined as the eigenfunctions of a particular weighted eigenvalue equation that involves the impedance matrix of the body. For conducting bodies they have the following properties:

- They are real or equiphasal, over the surface on which they exist.

- They form an orthogonal set over this surface.

- They diagonalize the generalized impedance matrix for this surface.

On the other hand, characteristic fields are defined to be the electromagnetic fields produced by the characteristic currents. They present the following important properties:

- The characteristic electric fields have equiphasal tangential components over the surface of the body.

- They form an orthogonal set over the radiation sphere.

- They diagonalize the scattering matrix for the body.

Modal solutions that can be obtained applying this theory have the next properties:

- Matrix inversion is not required for computation of the currents since generalized impedance matrix is diagonalized.

- Pattern synthesis can be accomplished without matrix inversion, since the scattering matrix is diagonalized.

- The current on the body can be controlled using the method of modal resonance.

Although the Theory of Characteristic Modes is extensively described in [30] and [31], this chapter includes, for the sake of completeness, a revision of its mathematical formulation. For illustration purposes, numerical examples for a very well-known antenna, such as the wire dipole, are presented. 


\subsection{Mathematical formulation of Characteristic Modes}

As explained in [30], the formulation of the Theory of Characteristic Modes for conducting bodies begins with the definition of an operator equation that relates the current $J$ on the surface $S$ of a conducting body with the tangential incident electric field $E^{i}$

$$
\left[L(J)-E^{i}\right]_{\tan }=0
$$

where the subscript "tan" denotes the tangential components on the surface $S$.

Figure 2.1 shows the typical scenario in the computation of characteristic modes, together with the system coordinates.

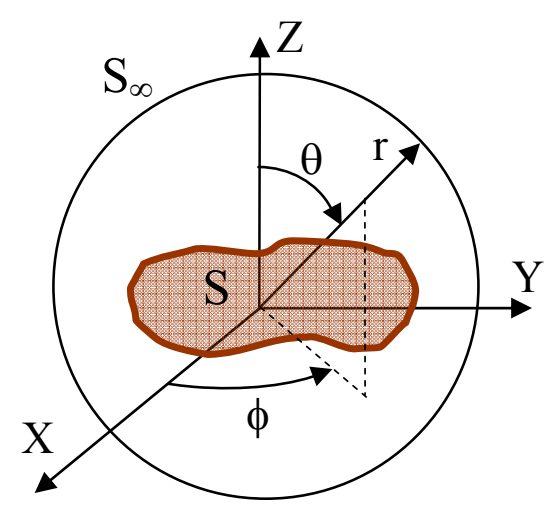

Figure 2.1 Scenario for the computation of characteristic modes, and system coordinates.

The operator $L$ in (2.1) is linear and it is defined by

$$
\begin{gathered}
L(J)=j \omega A(J)+\nabla \Phi(J) \\
A(J)=\mu \oiint_{S} J\left(r^{\prime}\right) \psi\left(r, r^{\prime}\right) d s^{\prime} \\
\Phi(J)=\frac{-1}{j \omega \varepsilon} \oiint_{S} \nabla^{\prime} \cdot J\left(r^{\prime}\right) \psi\left(r, r^{\prime}\right) d s^{\prime} \\
\psi\left(r, r^{\prime}\right)=\frac{\exp \left(-j k\left|r-r^{\prime}\right|\right)}{4 \pi\left|r-r^{\prime}\right|}
\end{gathered}
$$

where $r$ is a field point, $r^{\prime}$ is a source point, and $\varepsilon, \mu$ y $k$ are the permittivity, permeability and wavenumber, respectively, of free space. 
Physically, the term - $L(J)$ can be considered as the electric intensity at any point in space due to the current $J$ on the surface $S$. This means that the operator $L$ in (2.1) has the dimension of impedance

$$
Z(J)=[L(J)]_{\tan }
$$

As drawn from [30], the impedance operator $Z$ is complex, and it can be written as

$$
Z(J)=R(J)+j X(J)
$$

\subsubsection{Characteristic currents}

Going after the approach developed in [30], characteristic current modes can be obtained as the eigenfunctions of the following particular weighted eigenvalue equation

$$
X\left(\vec{J}_{n}\right)=\lambda_{n} R\left(\vec{J}_{n}\right)
$$

where $\lambda_{n}$ are the eigenvalues, $\vec{J}_{n}$ are the eigenfuncions or eigencurrents, and $R$ and $X$ are the real and imaginary parts a of the impedance operator $Z$.

It is known from reciprocity theorem that if $Z$ is a linear symmetric operator, then, its hermitian parts ( $R$ and $X$ ) will be real and symmetric operators. From this, it follows that all eigenvalues $\lambda_{n}$ in (2.8) are real, and all the eigencurrents $\vec{J}_{n}$ can be chosen real or equiphasal over the surface in which they are defined [30].

Moreover, the choice of $R$ as a weigh operator in (2.8) is responsible for the orthogonality properties of characteristic modes described in [30], which can be summarized as

$$
\begin{gathered}
\left\langle\overrightarrow{J_{m}^{*}}, R\left(\overrightarrow{J_{n}}\right)\right\rangle=\delta_{m n} \\
\left\langle\overrightarrow{J_{m}^{*}}, X\left(\overrightarrow{J_{n}}\right)\right\rangle=\lambda_{n} \delta_{m n} \\
\left\langle\overrightarrow{J_{m}^{*}}, Z\left(\overrightarrow{J_{n}}\right)\right\rangle=\left(1+j \lambda_{n}\right) \delta_{m n}
\end{gathered}
$$

where $\delta_{m n}$ is the Kronecher delta ( 0 if $m \neq n$, and 1 if $m=n$ ). 
Given that all eigencurrents $\vec{J}_{n}$ are real, the conjugate operation in equations (2.9), (2.10) and (2.11) can be omitted.

Consistent with (2.8), characteristic modes $\vec{J}_{n}$ can be defined as the real currents on the surface of a conducting body that only depend on its shape and size, and are independent of any specific source or excitation.

In practice, as explained in [31], to compute characteristic modes of a particular conducting body, equation (2.8) needs to be reduced to matrix form using Galerkin formulation

$$
[X] \vec{J}_{n}=\lambda_{n}[R] \vec{J}_{n}
$$

Note that matrix $R$ is positive semi-defined since the power radiated by a current $J_{n}$ on the surface of a conducting body, is $\left\langle{\overrightarrow{J_{n}}}^{*},[R] \overrightarrow{J_{n}}\right\rangle \geq 0$.

Next, eigencurrents $\vec{J}_{n}$ and eigenvalues $\lambda_{n}$ of the object are obtained by solving the generalized eigenvalue problem of (2.12) with standard algorithms [47]. See Appendix 1 for more details.

As an example, Figure 2.2 illustrates the current distribution at first resonance $(f=280 \mathrm{MHz})$ of the first four eigencurrents for a wire dipole of length $L=0.5 \mathrm{~m}$ and wire diameter $d=1 \mathrm{~mm}$, placed along the $Y$ axis. The wire is supposed to be a perfect conductor with no losses. Computation of modes was made using one hundred triangular functions for expansion and testing when applying method of moments.

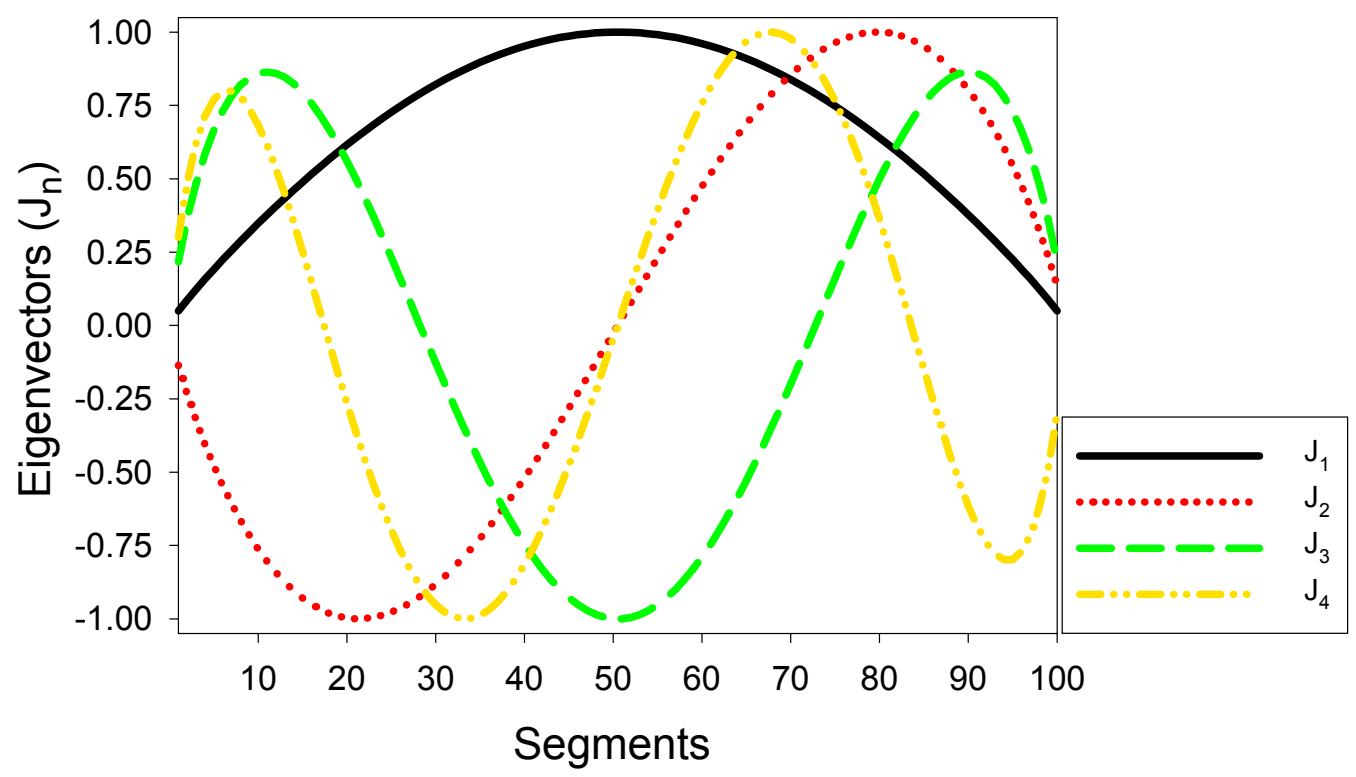

Figure 2.2 Normalized distribution at first resonance $(f=280 \mathrm{MHz}$ ) for the first four eigencurrents of a dipole of length $L=0.5 \mathrm{~m}$ and wire diameter $d=1 \mathrm{~mm}$. 
All currents in Figure 2.2 have been normalized to its maximum value in order to facilitate comparison. It can be observed that eigencurrents have sinusoidal form with nulls at the end points of the dipole. These eigencurrents also exhibit increasingly oscillatory nature as the order of the mode increases. First current mode presents one cycle, second current mode two cycles, and so on. Furthermore, modes can be classified in even modes $\left(J_{1}\right.$ and $\left.J_{3}\right)$ or odd modes $\left(J_{2}\right.$ and $\left.J_{4}\right)$ depending on the symmetry of their current distribution. It will be shown later, using the information provided by eigenvalues, that the fundamental mode $J_{1}$ resonates when the length of the dipole is approximately $L=\lambda / 2$. Similarly, higher order modes $J_{2}, J_{3}$ and $J_{4}$, resonate when the length of the dipole is $L=\lambda, L=3 \lambda / 2$ and $L=2 \lambda$, respectively.

It is worth mentioning that eigencurrents depend upon frequency, so they need to be recalculated at every frequency of interest. Figure 2.3 exemplifies the variation with frequency of the first four eigencurrents of the abovementioned wire dipole. It can be noticed that the oscillation of the modes increases with frequency in a significant way. This is because current modes couple to radiating spherical field modes with increasing azimuthal variation, $e^{j n \phi}, e^{j 2 n \phi}, e^{j 3 n \phi} \ldots .$.

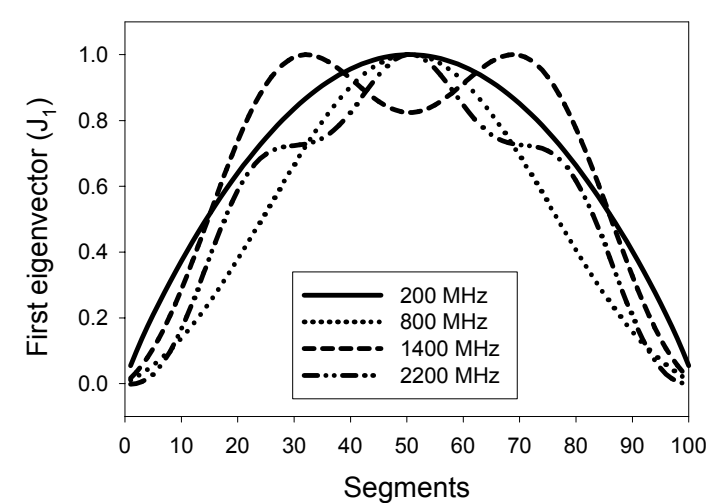

(a)

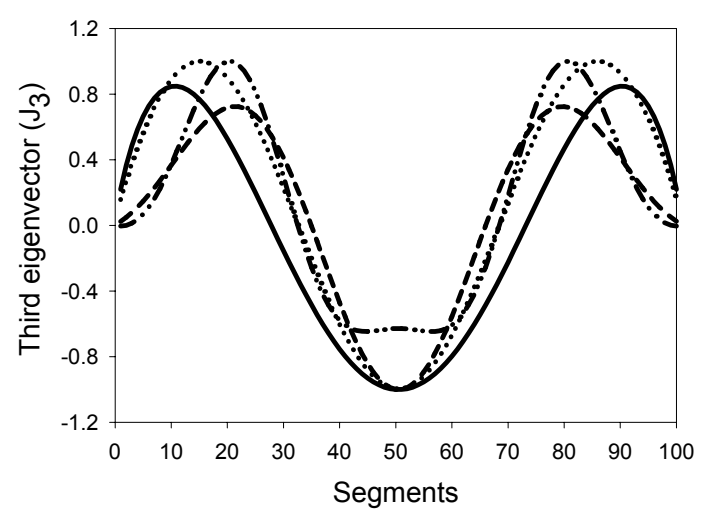

(c)

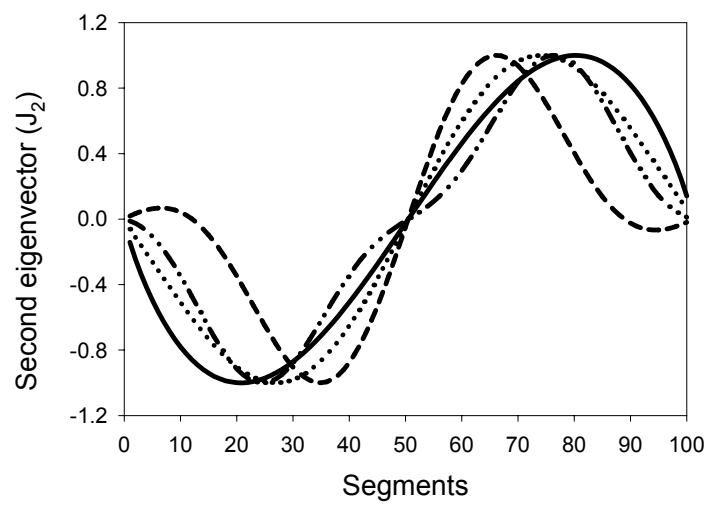

(b)

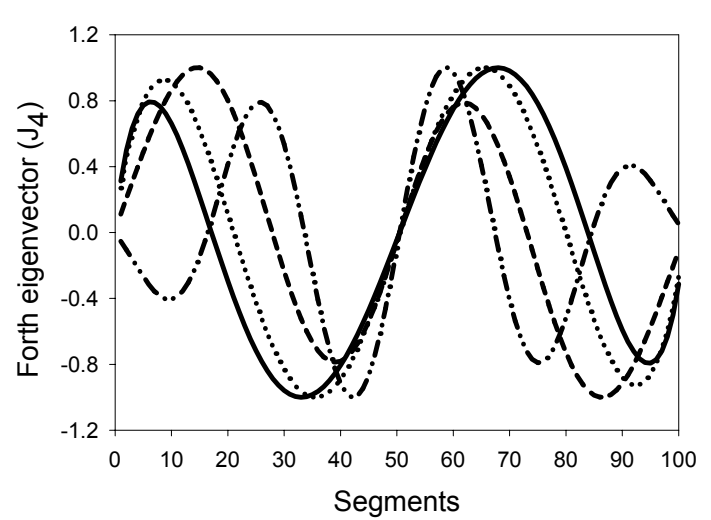

(d)

Figure 2.3 Current distribution of the eigenvectors of a dipole of length

$L=0.5 \mathrm{~m}$ and diameter $d=1 \mathrm{~mm}$ at four different frequencies: (a) First eigenvector $J_{1}$, (b) Second eigenvector $J_{2,}$ (c) Third eigenvector $J_{3 \prime}$ (d) Forth eigenvector $J_{4}$. 
Henceforth, all the results dealing with a dipole will refer to the previously described one of length $L=0.5 \mathrm{~m}$ and wire diameter $d=1 \mathrm{~mm}$. Since for electrically small conducting bodies few modes are required for accurate solutions, only the first four modes shown in Figure 2.2 are going to be considered from now on.

\subsubsection{Characteristic fields}

Electric fields $E_{n}$ produced by characteristic currents $J_{n}$ on the surface of a conducting body, are called characteristic fields [30]. From (2.8) it can be derived that these characteristic fields can be written as

$$
E_{n}\left(\vec{J}_{n}\right)=Z\left(\vec{J}_{n}\right)=R\left(\vec{J}_{n}\right)+j X\left(\vec{J}_{n}\right)=R\left(\vec{J}_{n}\right)\left(1+j \lambda_{n}\right)
$$

Then, from (2.13) it is extracted that characteristic fields are equiphasal, since they are $\left(1+j \lambda_{n}\right)$ times a real quantity. Orthoganality relationships for characteristic fields can be reached from characteristic currents by means of complex Poynting theorem

$$
\begin{aligned}
P\left(J_{m}, J_{n}\right) & =\left\langle J_{m}^{*}, Z J_{n}\right\rangle=\left\langle J_{m}^{*}, R J_{n}\right\rangle+j\left\langle J_{m}^{*}, X J_{n}\right\rangle \\
& =\oiint_{S^{\prime}} \vec{E}_{m} \times \vec{H}_{n}^{*} d s+j \omega \iiint_{\tau^{\prime}}\left(\mu \vec{H}_{m} \cdot \vec{H}_{n}^{*}-\varepsilon \vec{E}_{m} \cdot \vec{E}_{n}^{*}\right) d \tau \\
& =\left(1+j \lambda_{n}\right) \delta_{m n}
\end{aligned}
$$

If the surface $S$ is of finite extent and if $S^{\prime}$ is chosen to be the radiation sphere $S_{\infty}$, as shown in Figure 2.1 , it can be demonstrated that

$$
\frac{1}{\eta} \oiint_{S_{\infty}} \vec{E}_{n} \cdot \vec{E}_{m}^{*} d s=\delta_{m n}
$$

Hence, characteristic electric fields form an orthogonal set in the far field.

Figure 2.4 depicts the azimuthal radiation pattern ( $X Y$ plane) at first resonance $(280 \mathrm{MHz})$ for the modal electric fields $E_{\phi, n}$ produced by the current modes $J_{n}$ of the wire dipole. Note that the radiation is null in the axial direction of the dipole, because of the nulls in the current modes at the ends of the dipole. Also observe that the number of radiating lobes depends on the number of cycles of the associated current $J_{n}$ that increases with the order of the mode. 

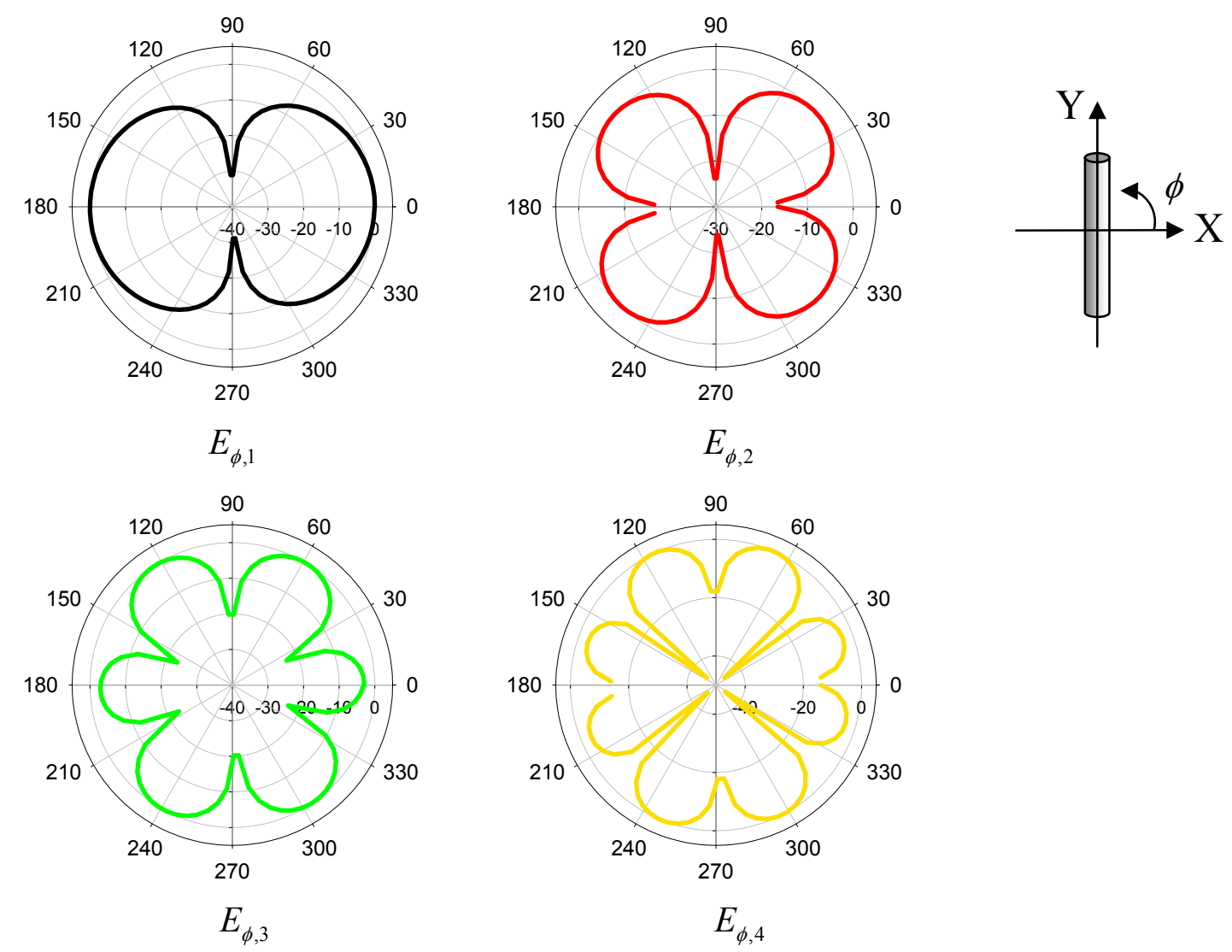

Figure 2.4 Azimuthal radiation pattern $\left(\theta=90^{\circ}\right)$ at $4 \mathrm{GHz}$ of the modal electric fields $E_{\phi, n}$ produced by the current modes $J_{n}$ of Figure 2.2 .

\subsubsection{Modal solutions}

Due to the already mentioned orthogonality properties over both the surface of the body and the enclosing sphere at infinity, characteristic modes radiate power independently of one another. Because of this attractive feature, characteristic modes can be used as a basis set in which to expand the unknown total current $J$ on the surface of the conducting body as

$$
J=\sum_{n} \alpha_{n} J_{n}
$$

where $\alpha_{n}$ are the coefficients to be determined.

Next step consist of substituting (2.16) into (2.1)

$$
\left[\alpha_{n} L\left(J_{n}\right)-E^{i}\right]_{\tan }=0
$$


Then, the inner product of (2.17) with each $J_{m}$ in turn is taken, giving the following set of equations

$$
\sum_{n} \alpha_{n}\left\langle J_{m}, Z J_{n}\right\rangle-\left\langle J_{m}, E^{i}\right\rangle=0
$$

where $m=1,2,3, \ldots \ldots$

Applying the orthogonality property of (2.11), equation (2.18) reduces to

$$
\alpha_{n}\left(1+j \lambda_{n}\right)=\left\langle J_{n}, E^{i}\right\rangle
$$

The right-hand term in (2.19) is called modal excitation coefficient [30]

$$
V_{n}^{i}=\left\langle J_{n}, E^{i}\right\rangle=\oint_{n} J_{n} \cdot E^{i} d s
$$

Once coefficients $\alpha_{n}$ have been obtained from (2.19), equation (2.16) can be expressed as

$$
J=\sum_{n} \frac{V_{n}^{i} J_{n}}{1+j \lambda_{n}}
$$

Note the modal excitation coefficient, $V_{n}^{i}$, accounts for the way the position, magnitude, and phase of the applied excitation influence the contribution of each mode to the total current $J$. Consequently, the product $V_{n}^{i} J_{n}$ in (2.21) models the coupling between the excitation and the $\mathrm{n}^{\text {th }}$ mode, and it determines if a particular mode is excited by the antenna feed or incident field.

However, the total current in (2.21) also depends on $\lambda_{n}$, the eigenvalue associated to the $\mathrm{n}^{\text {th }}$ characteristic current mode. Eigenvalues are of utmost importance because its magnitude gives information about the resonance frequency and radiating properties of the different current modes.

Next section explains in detail how to make the most of the information provided by eigenvalues in order to obtain physical insight into the radiation phenomena taking place on the antenna. 


\subsection{Physical interpretation of Characteristic Modes}

The easiest way to understand how the magnitude of eigenvalues is related to the radiation of modes is analyzing the complex power balance yielded by equation (2.14). From this equation it can be deduced that, by definition, the power radiated by modes is normalized to unit value. In contrast, reactive power is proportional to the magnitude of the eigenvalues.

In general, eigenvalues $\lambda_{n}$ range from $-\infty$ to $+\infty$. Considering that a mode is at resonance when its associated eigenvalue is zero, it is inferred that the smaller the magnitude of the eigenvalue is, the more efficiently the mode radiates when excited. Additionally, the sign of the eigenvalue determines whether the mode contributes to store magnetic energy $\left(\lambda_{n}>0\right)$ or electric energy $\left(\lambda_{n}<0\right)$.

Figure 2.5 shows the variation with frequency of the eigenvalues $\lambda_{n}$ of the four current modes of the wire dipole studied in previous sections. It is observed that eigenvalues start being negative, next they resonate $\left(\lambda_{n}=0\right)$, and at the end they keep a small uniform positive value. Hence, mode $J_{l}$ resonates at 290 $\mathrm{MHz}$, mode $J_{2}$ at $590 \mathrm{MHz}$, mode $J_{3}$ at $890 \mathrm{MHz}$, and mode $J_{4}$ resonates out of the analyzed frequency band.

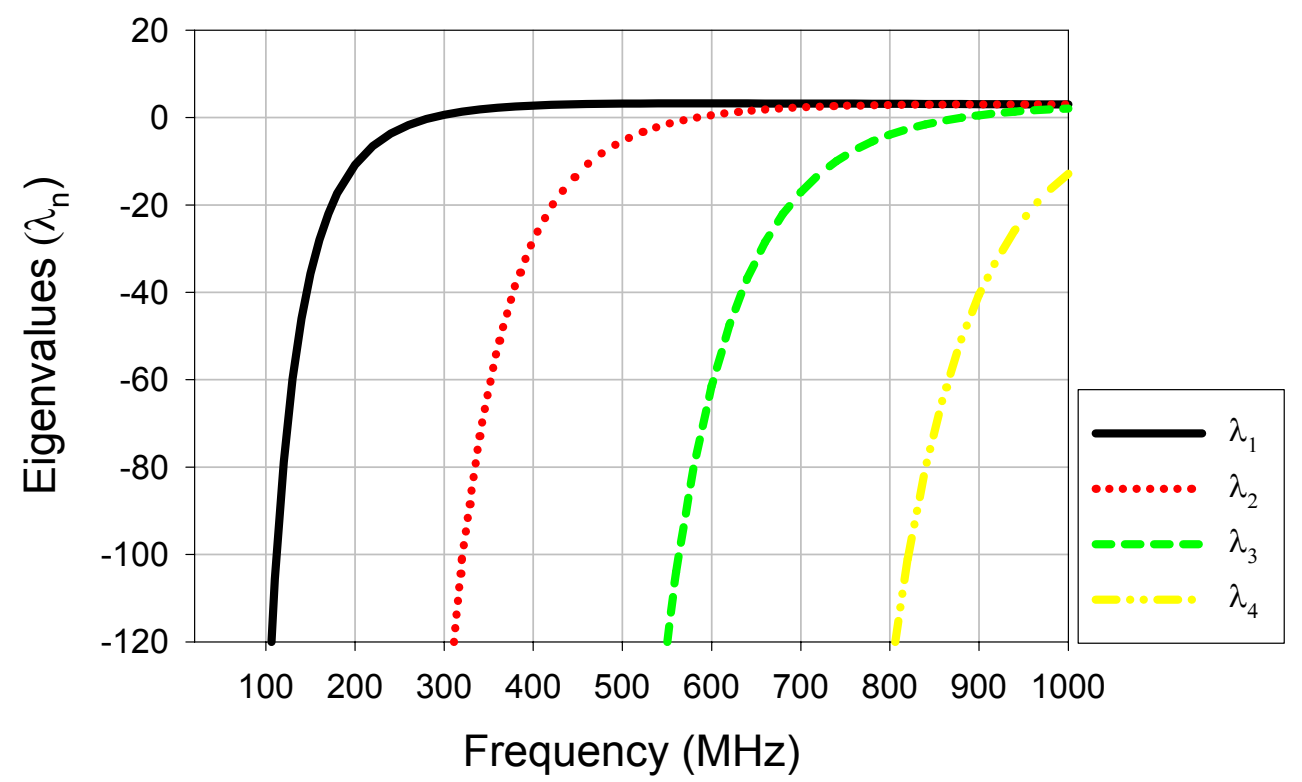

Figure 2.5 Variation with frequency of the eigenvalues $\lambda_{n}$ associated to the current modes $J_{n}$ of the dipole depicted in Figure 2.2 . 
It is important to highlight that the real nature of characteristic modes derived from (2.8) constitutes an advantage in comparison with complex natural modes directly obtained from impedance matrix $[Z]$. Working with complex basis functions deals to complexity increase in computation, since it is necessary to give different treatment to the real and imaginary parts of the current to get to accurate results [48]. Another drawback of natural modes is that their eigenvalues are also complex, thus they are not so easy to analyze and explain physically.

\subsubsection{Modal significance}

As exposed before, an analysis of the eigenvalue variation with frequency is very useful for antenna design as it brings information about the resonance frequency of modes. Nevertheless, in practice other alternative representations of the eigenvalues are preferred. Since the modal expansion of the current described in (2.21) is inversely dependent upon eigenvalues, it seems more logical to analyze the variation of the term

$$
M S_{n}=\left|\frac{1}{1+j \lambda_{n}}\right|
$$

better than the variation of the isolated eigenvalue.

The term presented in (2.22) is usually called modal significance $\left(M S_{n}\right)$ and it represents the normalized amplitude of the current modes [41]. This normalized amplitude only depends on the shape and size of the conducting object, and does not account for excitation.

Figure 2.6 depicts the variation with frequency of the modal significance related to the current modes $J_{n}$ of the dipole. Now, the resonance of each mode can be identified by a maximum value of one in the modal significances curves. This means that the nearest the curve is to its maximum value, the most effectively the associated mode contributes to radiation.

Then, the radiating bandwidth of a mode can be established according to the width of its modal significance curve near the maximum point. This radiating bandwidth constitutes an important figure-of-merit to determine the radiating performance of modes.

Let us define the radiating bandwidth of a mode $B W_{n}$ as the range of frequencies within which the power radiated by the mode is not less than onehalf the power radiated at resonance. 
Translated to modal significance curves, half-power $(H P)$ at resonance corresponds to a reduction of the normalized current by a factor $\sqrt{2}$

$$
M S_{H P_{-} n}=\left|\frac{1}{1+j \lambda_{n}}\right|=\frac{1}{\sqrt{2}}=0.707
$$

Hence, the radiation bandwidth of a mode $B W_{n}$ can be expressed as a fraction of the frequency difference (upper minus lower) over the resonance frequency of the mode

$$
B W_{n}=\frac{f_{U}-f_{L}}{f_{\text {res }}}
$$

where $f_{\text {res }}$ is the resonance frequency of the mode, and $f_{U}$ and $f_{L}$ are the upper and lower limits, respectively, according to the previous definition.

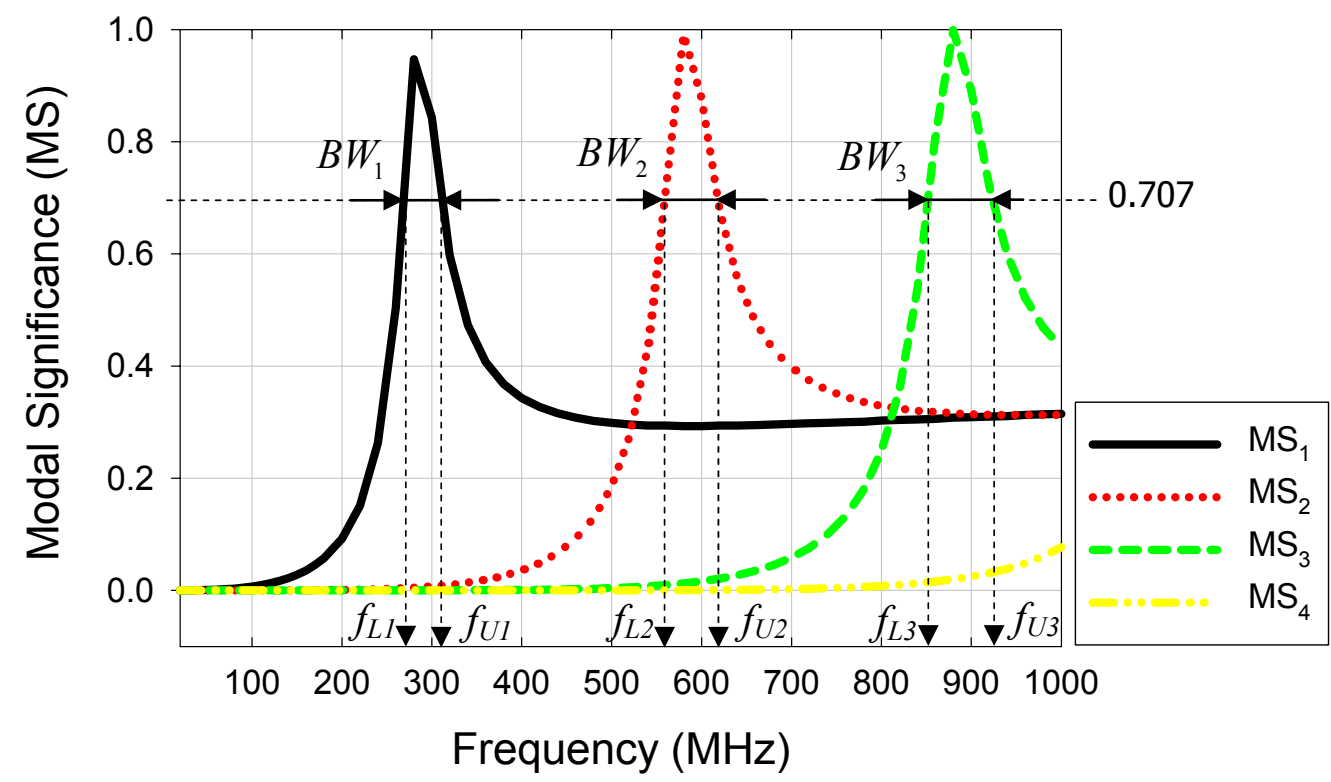

Figure 2.6 Variation with frequency of the modal significance $\left(M S_{n}\right)$ related to the current modes $J_{n}$ of the dipole sketched in Figure 2.2 .

Moreover, a modal quality factor can be defined from the fractional bandwidth as

$$
Q_{\text {rad }, n}=\frac{1}{B W_{n}}
$$

provided $Q_{r a d, n} \gg>1$. 
The quality factor of a resonant mode measures how sharp its resonance is. Then, the higher the $Q_{\text {rad, }, n}$ the sharper the resonance of the mode is, and the narrower the radiating bandwidth results.

On the basis of the information provided by Figure 2.6 Table 2.1 summarizes the resonance frequency, half-power radiating bandwidth and modal quality factor of the first three modes of the dipole.

\begin{tabular}{|l|c|c|c|c|c|}
\cline { 2 - 6 } \multicolumn{1}{c|}{} & $f_{\text {res }}(\mathrm{MHz})$ & $f_{L}(\mathrm{MHz})$ & $f_{U}(\mathrm{MHz})$ & $B W_{n}(\%)$ & $Q_{\text {rad }, n}$ \\
\hline Mode $J_{1}$ & 290 & 270 & 310 & 13.79 & 7.25 \\
\hline Mode $J_{2}$ & 590 & 560 & 620 & 10.16 & 9.84 \\
\hline Mode $J_{3}$ & 890 & 855 & 925 & 7.86 & 12.72 \\
\hline
\end{tabular}

Table 2.1. Resonance frequency, radiating bandwidth and quality factor of the first three modes of the dipole.

The modal quality factor proposed in (2.25) presents the advantage of simplicity and ease of calculation. To validate its accuracy, results in Table 2.1 for $Q_{r a d, n}$ are going to be compared with values provided by alternative definitions of the quality factor.

A classical formula to obtain the quality factor due to a current $I$, is that based on the Foster reactance theorem [49]-[50]

$$
Q_{\text {Foster }}=\frac{\left[I^{*}\right]^{t}\left[\omega \frac{d[X]}{d \omega} \pm[X]\right][I]}{\left[I^{*}\right]^{t}\left[[Z]+\left[Z^{*}\right]^{t}\right][I]}
$$

where either the + or - value is chosen to give the higher $Q$.

Similarly, Harrington and Mautz in [38] approximated the quality factor of a current $I$ as

$$
Q=\frac{\omega}{2} \frac{\left[\tilde{I}^{*}\right]\left[\frac{d X}{d \omega}\right][I]}{\left[\tilde{I}^{*}\right][R][I]}
$$

where $R$ and $X$ are the real, and imaginary parts of the impedance matrix of the body, respectively.

Note that although (2.27) is similar to (2.26), they are slightly different. In $(2.27), \pm[X]$ has been neglected, since for high $Q$ values, the term obtained from $\omega(\partial[X] / \partial \omega)$ is usually larger than that obtained from $[X]$. 
Then, a modal quality factor could be obtained from either (2.26) or (2.27), if the current $I$ were a characteristic current $J_{n}$.

Nevertheless, the values for the modal quality factor presented in [38] have been obtained by solving the following eigenvalue equation directly derived from (2.27)

$$
\left[\omega \frac{d X}{d \omega}\right]\left[J_{n}\right]=Q_{n}[R]\left[J_{n}\right]
$$

where $Q_{n}$ are the eigenvalues, and $\left[J_{n}\right]$ are the eigencurrents.

Finally, in [38] an interpretation of a modal $Q_{n}$ made in terms of the frequency variation of the eigenvalues is also proposed

$$
Q_{n, \text { Harrington }} \approx \frac{\omega}{2} \frac{d \lambda_{n}}{d \omega}
$$

The quality factor defined in (2.29) measures the frequency sensitivity of a modal current, so it should be equivalent to that previously defined in (2.25).

Table 2.2 compares the results obtained for the modal quality factor of the first three modes of the wire dipole at resonance $\left(\omega=\omega_{0}\right)$, using $(2.25),(2.26)$ and (2.29).

\begin{tabular}{|c|c|c|c|}
\cline { 2 - 4 } \multicolumn{1}{c|}{} & $Q_{n, \text { rad }}$ & $Q_{n, \text { Foster }}$ & $Q_{n, \text { Harrington }}$ \\
\hline Mode $J_{1}$ & 7.25 & 7.32 & 7.08 \\
\hline Mode $J_{2}$ & 9.84 & 10.17 & 9.97 \\
\hline Mode $J_{3}$ & 12.72 & 12.73 & 12.38 \\
\hline
\end{tabular}

Table 2.2. Modal quality factor for the first three modes of the dipole, computed using (2.25), (2.26) and (2.29).

Results in Table 2.2 demonstrate that the definitions of the modal quality factor in (2.25), (2.26) and (2.29) are equivalent, since they provide very similar values.

In Chapter 5 further discussion about the modal quality factor of broad band radiators will be presented.

Next section deals with another representation of the eigenvalues that is based on the use of characteristic angles. 


\subsubsection{Characteristic angle}

Characteristic angles are defined in [51] as:

$$
\alpha_{n}=180^{\circ}-\tan ^{-1}\left(\lambda_{n}\right)
$$

From a physical point of view, a characteristic angle models the phase angle between a characteristic current $J_{n}$ and the associated characteristic field $E_{n}$.

Figure 2.7 presents the variation with frequency of the characteristic angles $\left(\alpha_{n}\right)$ associated to the current modes of the dipole. Observe that a mode resonates when $\lambda_{n}=0$, that is, when its characteristic angle $\alpha_{n}$ is $180^{\circ}$.

Therefore, when the characteristic angle is close to $180^{\circ}$ the mode is a good radiator, while when the characteristic angle is near $90^{\circ}$ or $270^{\circ}$ the mode mainly stores energy. Thus, the radiating bandwidth of a mode can be more or less deduced from the slope near $180^{\circ}$ of the curve described by characteristic angles.

To compute the radiating bandwidth of modes from characteristic angles using (2.24), it is necessary to determine which values of characteristic angle correspond with one-half the power radiated at resonance. From (2.23) it can be deduced that there are two eigenvalues, $\lambda_{n}=1$ and $\lambda_{n}=-1$, that produce a modal significance of 0.707 . These eigenvalues generate characteristic angles of $135^{\circ}$ and $225^{\circ}$. Then, when computing the radiating bandwidth of modes using characteristic angles, $f_{H}$ and $f_{L}$ in equation (2.24) are the frequencies at which characteristic angles are $135^{\circ}$ and $225^{\circ}$, respectively.

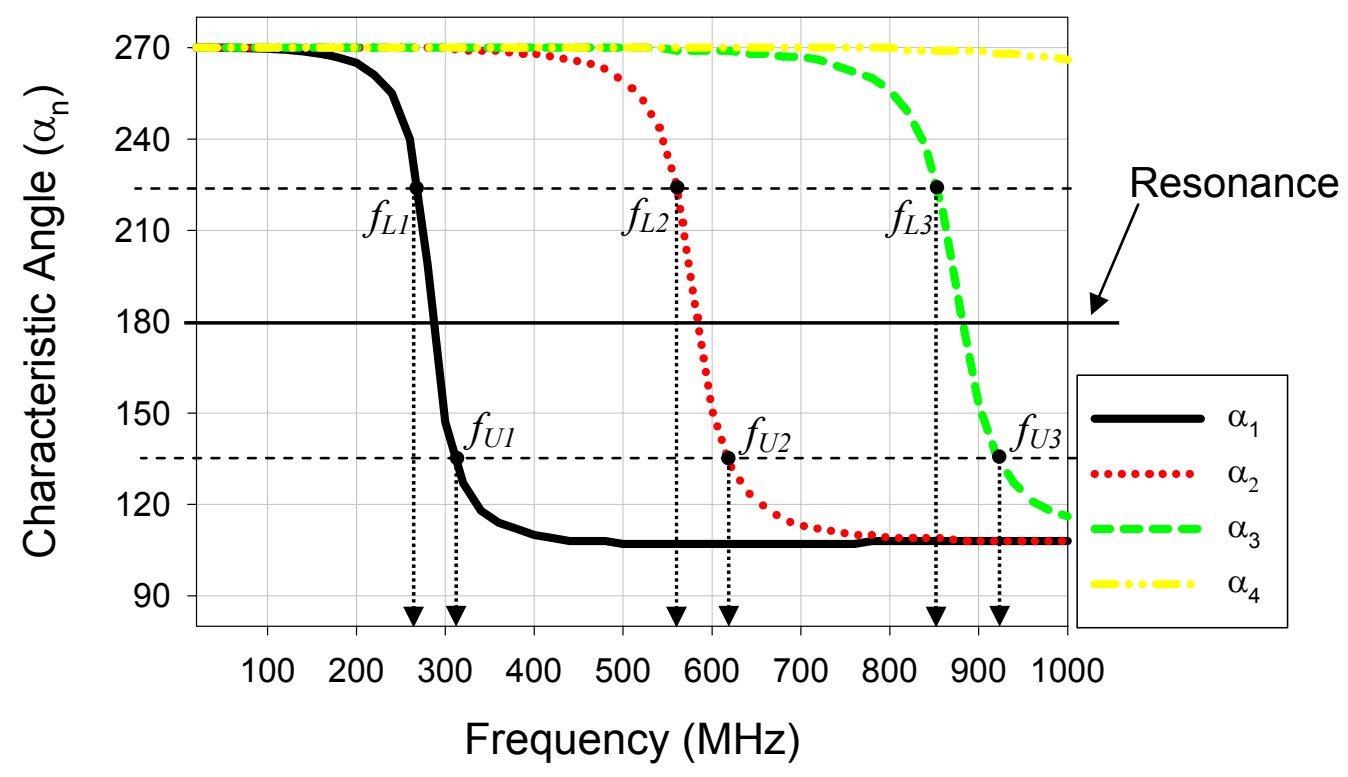

Figure 2.7 Variation with frequency of the characteristic angle $\alpha_{n}$ associated to the current modes $J_{n}$ of the dipole sketched in Figure 2.2 
Although the information given by Figure 2.7 could have also been extracted from Figure 2.5 or Figure 2.6 characteristic angle representation is often preferred as it is the most intuitive one.

In next chapter it will be shown that although the behaviour shown in Figure 2.7 is the typical behaviour of characteristic angles, there are also special nonresonant inductive modes with angles below $180^{\circ}$ at every frequency.

\subsection{Excitation of Characteristic Modes}

Once current distribution, resonance frequency and radiating bandwidth of characteristic modes have been determined, next step is to find out which modes are excited when an excitation source is placed over the wire dipole.

In order to determine how each mode contributes to the input bandwidth, the input admittance $Y_{\text {in }}$ of the antenna is studied. For a voltage excitation of $1 \mathrm{~V}$, the input admittance of the antenna $Y_{\text {in }}$ can be easily calculated, as it is equal to the current $J$ in equation (2.21) sampled at feed point $P$

$$
Y_{i n}=\frac{J(P)}{1 V}=\sum_{n} \frac{V_{n}^{i} \cdot J_{n}(P)}{1+j \lambda_{n}}
$$

In the same manner as in (2.16), the complex input admittance $\left(Y_{i n}\right)$ can be expressed as sum of complex eigenadmittances, or modal admittances $\left(Y_{n}\right)$ as follows

$$
Y_{i n}=\sum_{n} Y_{n}=\sum_{n} G_{n}+j B_{n}=\sum_{n} \frac{V_{n}^{i} \cdot J_{n}(P)}{1+\lambda_{n}{ }^{2}}-j \frac{V_{n}^{i} \cdot J_{n}(P) \cdot \lambda_{n}}{1+\lambda_{n}{ }^{2}}
$$

Just to conclude the modal characterization of the dipole used in this chapter as example, a delta gap source is going to be placed at different locations to investigate the excitation of modes. In first place, the case of the centre fed dipole is going to be considered. Figure 2.8 shows the complex input admittance $Y_{i n}$ of this centre fed dipole together with the complex modal admittances $Y_{n}=G_{n}+j B_{n}$ associated to the current modes of Figure 2.2. As it was be expected, only even modes, $J_{1}$ and $J_{3}$ are excited, since odd modes, $J_{2}$ and $J_{4}$ present zero current amplitude at the centre of the dipole. Mode $J_{I}$ dominates at lowest frequencies and it is responsible for the resonance at $290 \mathrm{MHz}$, while the resonance at $890 \mathrm{MHz}$ is due to mode $J_{3}$ that is dominant at highest frequencies. In contrast, in the frequency band that ranges from $400 \mathrm{MHz}$ to $600 \mathrm{MHz}$ there is no dominant mode, and the antiresonance that appears at 525 $\mathrm{MHz}$ is caused by the interaction of modes $J_{1}$ and $J_{3}$.

Let us consider now a delta gap source located at a quarter of the length of the dipole. The contribution of the modal admittances $Y_{n}$ to the input admittance $Y_{i n}$ of the dipole, for this new location of the source, is presented in Figure 2.9 
It should be emphasized that for the case of the non-centre fed dipole, both odd and even modes are excited. Mode $J_{I}$ is responsible for the first resonance at $220 \mathrm{MHz}$. Modes $J_{2}$ and $J_{3}$ cause the resonances at $600 \mathrm{MHz}$ and $800 \mathrm{MHz}$, respectively. Also from Figure 2.9 it can be deduced that the first antiresonance at $300 \mathrm{MHz}$ is due to the interaction of modes $J_{1}$ and $J_{2}$, and the second antiresonance at $700 \mathrm{MHz}$ turns out from the combination of modes $J_{l_{1},} J_{2}$ and $J_{3}$.

These results confirm that the resonances of an antenna can be directly attributed to radiating modes, while antiresonances are caused by the combination of two or more modes. Furthermore, these results probe that a resonant dipole $(L \approx 0.5 \lambda)$ can be analyzed in a wide frequency band (from $L=0.1 \lambda$ to $L=1.6 \lambda$ ) just using the first four radiating modes.

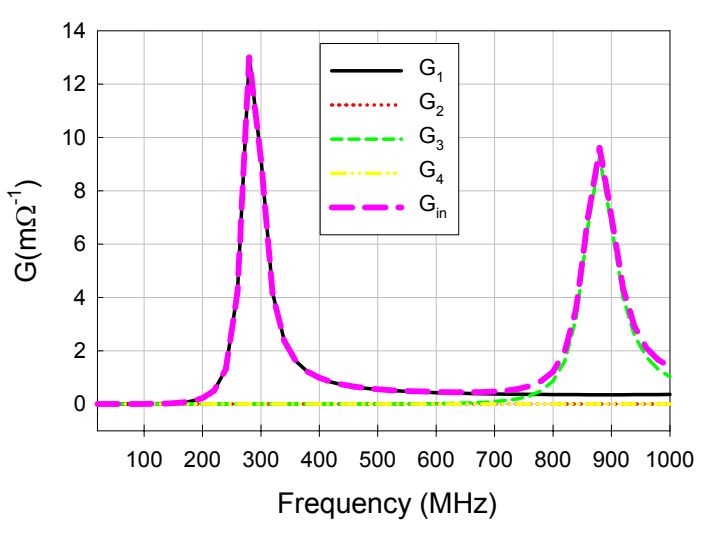

(a)

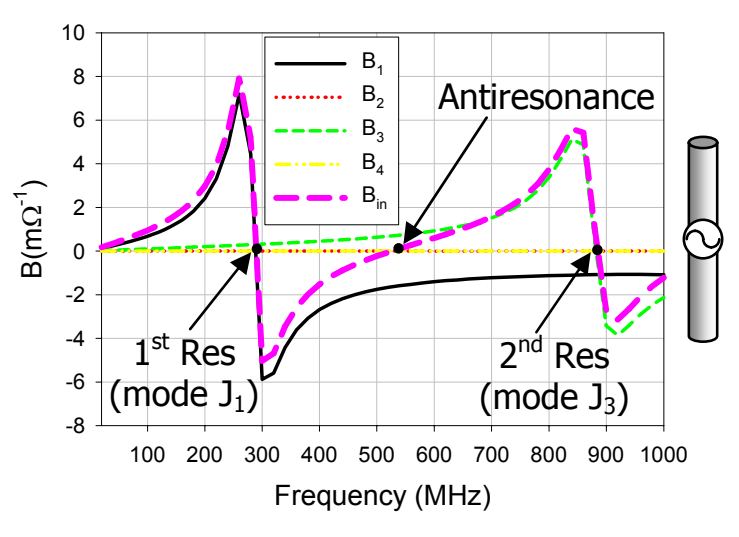

(b)

Figure 2.8 Representation of the input admittance $Y_{\text {in }}$ and modal admittances $Y_{n}$ of the centre fed dipole: (a) Real part $(G)$, and (b) Imaginary part (B).

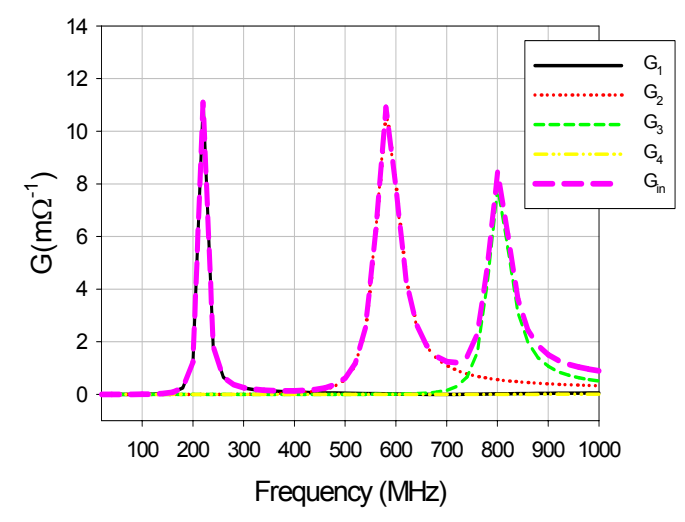

(a)

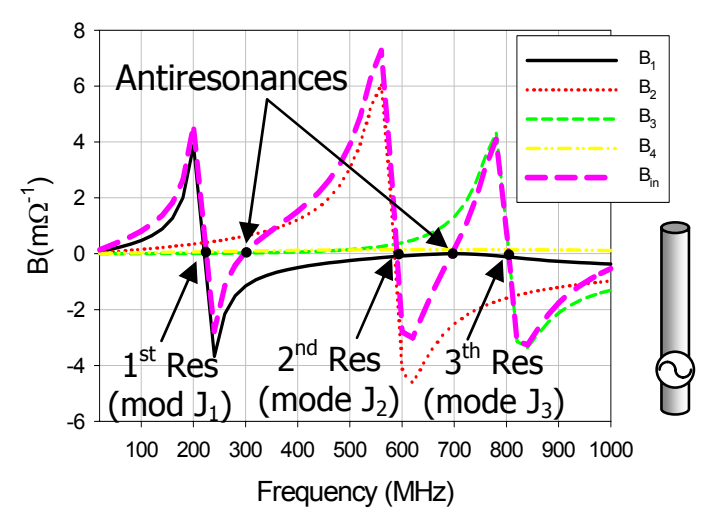

(b)

Figure 2.9 Representation of the input admittance $Y_{\text {in }}$ and modal admittances $Y_{n}$ of a dipole fed at one quarter of its length: (a) Real part $(G)$, and

(b) Imaginary part $(B)$. 


\subsection{Mode resonance by reactive loading}

Reactive loading has been extensively used for the last four decades not only for designing wire antennas [52]-[54], but also other types of antennas such as microstrip patches [55]-[57] or planar monopoles [58]-[59].

First publications dealing with reactive loading can be attributed to the most mentioned author in this thesis, R. F. Harrington [60]-[62]. After some preliminary works, Harrington proposed the use reactive loading in conjunction with the Theory of Characteristic modes for the control of radiation and scattering of conducting bodies [38]-[39]. When reactive loading is combined with characteristic modes it gains a more practical perspective since total control of the electromagnetic behaviour of the antenna can be achieved.

\subsubsection{Continuous loading}

Using the method reported in [38], any current mode on the body can be made resonant at the desired frequency by adding reactive loads. Basically, when applying this method, the matrix equation described in (2.12) is substituted by

$$
\left[X+X_{L}\right] \vec{J}_{n}=\lambda_{n}[R] \vec{J}_{n}
$$

where $\left[X_{L}\right]$ is the imaginary part of the load impedance matrix $\left[Z_{L}\right]$, which is a pure reactance $\left(R_{L}=0\right)$. Moreover, if Galerkin formulation is used to discretize the problem $\left[X_{L}\right]$ will be a diagonal matrix

$$
\left[X_{L}\right]=\left[\begin{array}{cccc}
X_{L 1} & 0 & 0 & \ldots . . \\
0 & X_{L 2} & 0 & \ldots . . \\
0 & 0 & X_{L 3} & \ldots . . \\
\ldots \ldots & \ldots \ldots . & \ldots \ldots & \ldots . . .
\end{array}\right]
$$

This new definition of the eigenvalue problem established in (2.33) accounts for reactive loading, but it does not alter the properties of characteristic modes. So, when solving equation (2.33) eigenvalues are still real, and eigencurrents can be chosen real. Orthogonality relationships of modes also remain unchanged.

As explained in section 2.3, a mode $J_{n}$ resonates when its eigenvalue is $\lambda_{n}=0$. This means that at resonance, the left-hand side of (2.33) is zero. Hence, a current mode $J_{n}$ can be forced to resonate at any frequency just by making the right-hand side of (2.33) equal to zero at this frequency [38]. 
This artificial resonance can be accomplished by adding an appropriate reactive load matrix $\left[X_{L}\right]$ whose diagonal elements can be obtained from

$$
X_{L i} \cdot J_{n_{i}}=-\left([X] \cdot\left[J_{n}\right]\right)_{i}
$$

with $i=1,2,3, \cdots$. The notation $\left([X] \cdot\left[J_{n}\right]\right)_{i}$ means the $i^{\text {th }}$ element of the column matrix $[X] \cdot\left[J_{n}\right]$, and $J_{n}$ is the desired current mode.

To exemplify this procedure, the fundamental mode $J_{l}$ of the wire dipole that has been used as example along this chapter, is going to be made resonant at $120 \mathrm{MHz}$ using reactive loading. Figure 2.10 shows the distribution of reactive loads $X_{L i}$ required along the one hundred segments of the wire dipole. These reactive loads have been computed according to (2.35), using the current mode $J_{1}$, and the imaginary part of the generalized impedance matrix of the dipole $[X]$ at $120 \mathrm{MHz}$. It can be observed that the distribution of loads is symmetrical and that all loads are positive. It is also observed that this load distribution is nearly uniform at the centre of the dipole, while at the ends it presents high values.

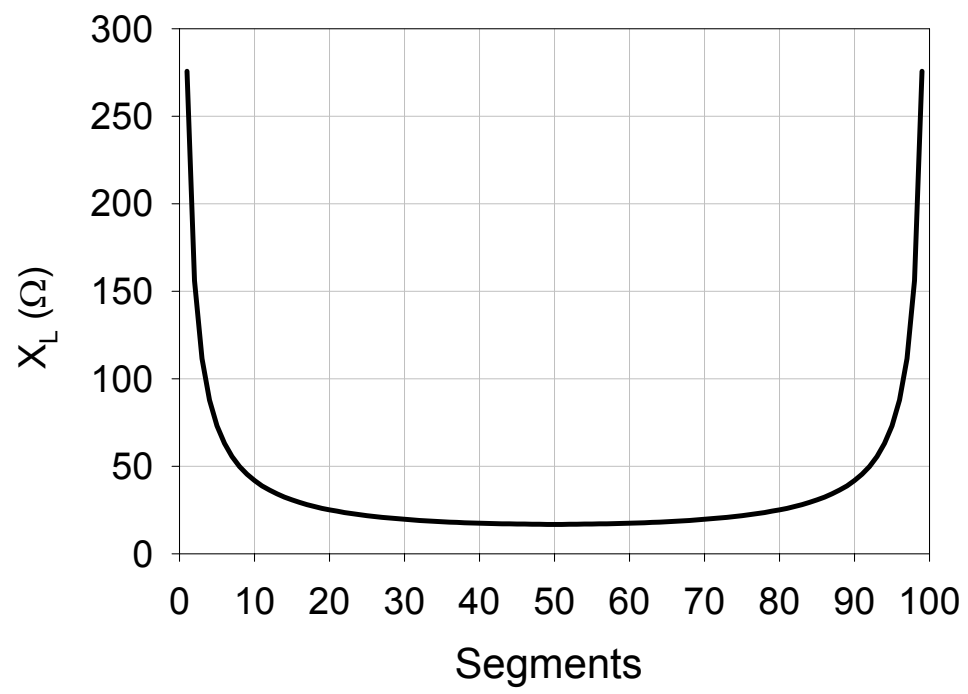

Figure 2.10 Distribution along the wire dipole of the reactive loads required to make mode $\mathrm{J}_{1}$ resonant at $120 \mathrm{MHz}$.

Once the desired load distribution to make mode $J_{l}$ resonant at $120 \mathrm{MHz}$ has been found, next step is loading the dipole with the corresponding reactances. Physically, the distributed load shown in Figure 2.10 can be modelled by lumped reactances placed at the different segments of the dipole. Numerically, the effect of the loading is included by replacing the generalized matrix of the dipole $[Z]$ by $\left[Z+j X_{L}\right]$, where $j X_{L}$ is the load matrix. 
Figure 2.11 depicts the characteristic angle variation with frequency for the first four modes of the loaded dipole. As expected, mode $J_{1}$ resonates at 120 $\mathrm{MHz}$. However, the resonance frequencies of the rest of modes have also been shifted to lower frequencies due to the effect of the loading. Another effect is the variation of the slope of characteristic angles curves, that now are steepest than before.

For brevity, the current distributions of the loaded modes are not going to be presented here since they are very similar to those depicted in Figure 2.2 and they do not provide any relevant information.

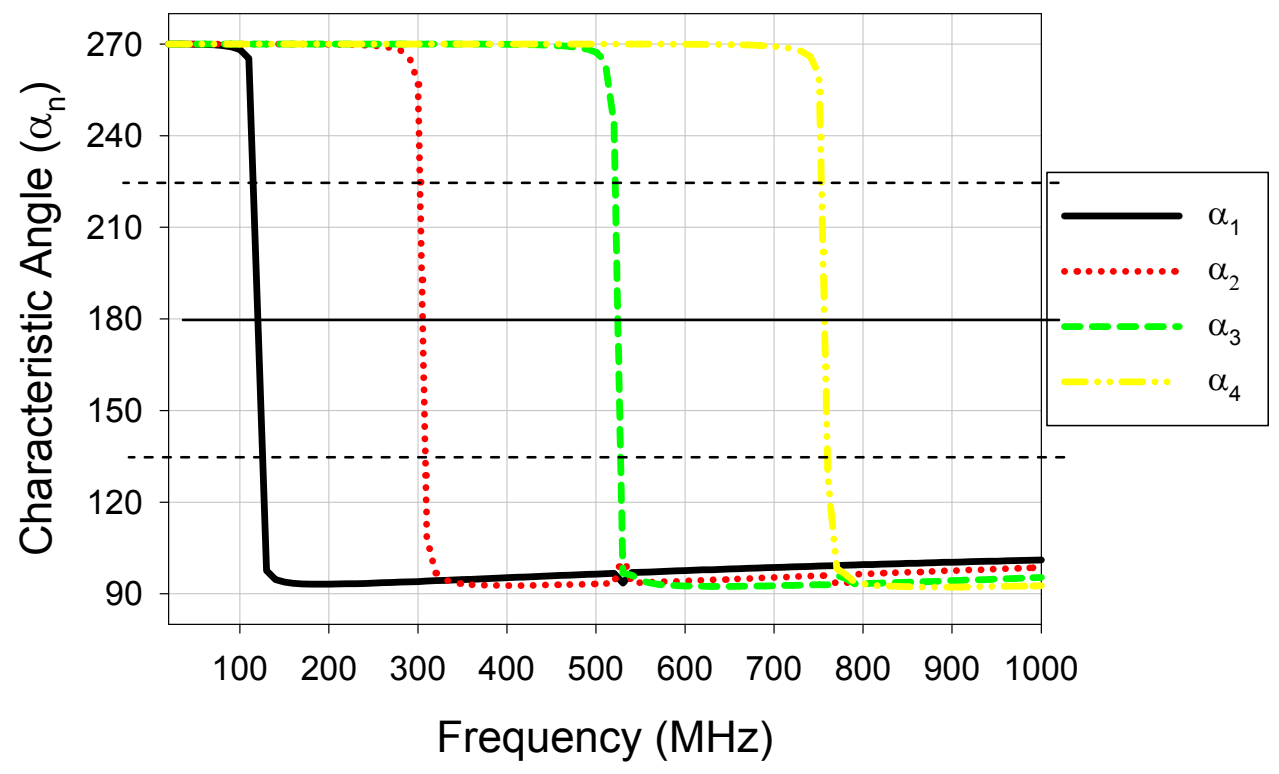

Figure 2.11 Characteristic angle variation with frequency for the four first current modes of a dipole of length $L=0.5 \mathrm{~m}$ when it is loaded with the distribution of reactances shown in Figure 2.10.

Table 2.3 illustrates the resonance frequency, radiating bandwidth and quality factor of the first four modes of the loaded dipole. The comparison of data in Table 2.3 with those obtained in Table 2.1 for the not loaded dipole yields several conclusions. The most obvious one is that all modes have been shifted to lowest frequencies. The resonance frequency has been shifted 170 $\mathrm{MHz}$ for mode $J_{1}, 285 \mathrm{MHz}$ for mode $J_{2}, 365 \mathrm{MHz}$ for mode $J_{3}$, and more than $400 \mathrm{MHz}$ for mode $J_{4}$. Thus, it seems that the higher the order of the mode is, the more affected its resonance frequency is by the loading. Another important conclusion is that the radiating bandwidth of modes has diminished because of the effect of the loading. This radiating bandwidth reduction is caused by the steepest slope that present the characteristic angle curves of the loaded dipole. 


\begin{tabular}{|c|c|c|c|c|c|}
\cline { 2 - 6 } \multicolumn{1}{c|}{} & $f_{\text {res }}(\mathrm{MHz})$ & $f_{L}(\mathrm{MHz})$ & $f_{U}(\mathrm{MHz})$ & $B W_{n}(\%)$ & $Q_{\text {rad, } n}$ \\
\hline Mode $J_{1}$ & 120 & 115 & 125 & 8.3 & 12 \\
\hline Mode $J_{2}$ & 305 & 300 & 310 & 3.2 & 30.5 \\
\hline Mode $J_{3}$ & 525 & 520 & 530 & 1.9 & 52.5 \\
\hline Mode $J_{4}$ & 757 & 755 & 760 & 0.7 & 151.4 \\
\hline
\end{tabular}

Table 2.3. Resonance frequency, radiating bandwidth and quality factor of the four first modes of the loaded dipole.

As a rule, by using reactive loading a more compact antenna can be achieved at the cost of loosing impedance bandwidth. This can be verified by studying the input impedances shown in Figure 2.12 , which correspond with the centre-fed dipole analyzed in section 2.4, and the same centre-fed dipole, loaded with the distribution of loads of Figure 2.10 For the case of the not loaded dipole the frequency separation between first and second resonances is $600 \mathrm{MHz}$. As was demonstrated in section 2.4, first and second resonances are associated to modes $J_{1}$ and $J_{3}$, respectively. Likewise, resonances of the loaded dipole are caused by modes $J_{l}$ and $J_{3}$, with a separation between them of 405 $\mathrm{MHz}$.

The distance between two consecutive resonances is critical, as it determines the location of the antiresonance that results from the interaction of the inductive modes that have already resonated, with the capacitive modes that will resonate next. Antiresonances are characterized by very high resistance values that degrade the impedance bandwidth of the antenna. In general, the closest an antiresonance is to a resonance, the sharpest is the antiresonance, and in consequence, the more degraded results the impedance bandwidth around the resonance.

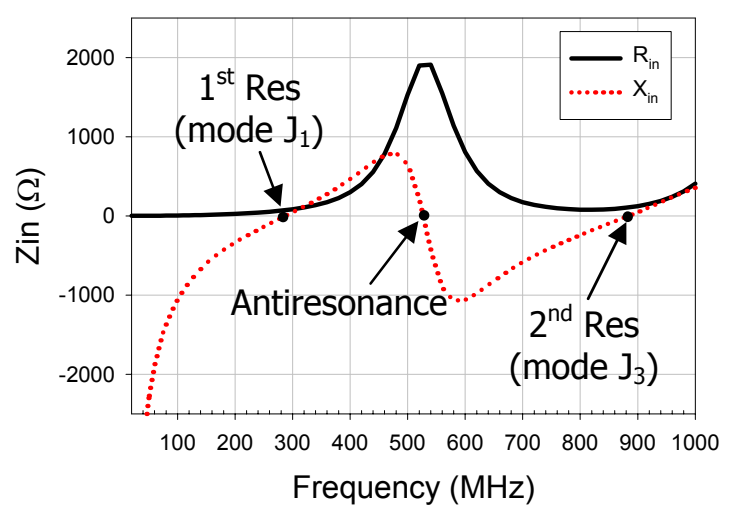

(a) Centre-fed dipole

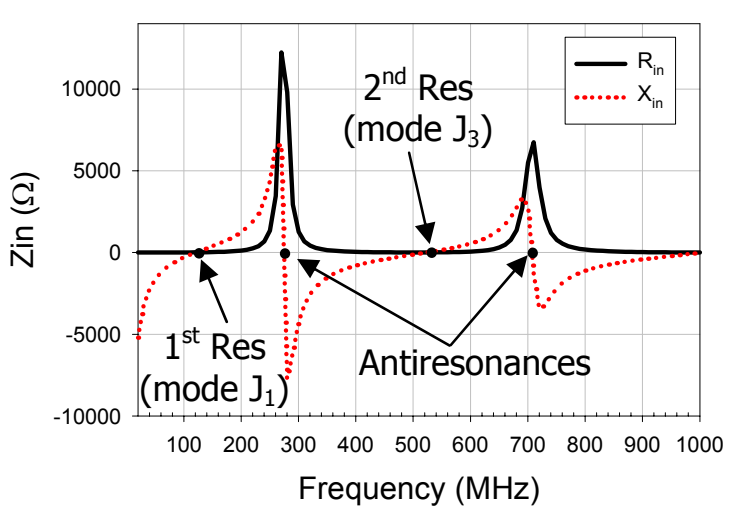

(b) Loaded centre-fed dipole

Figure 2.12 Input impedance of: (a) Centre-fed dipole of length $L=0.5 \mathrm{~m}$. (b) Centre-fed dipole of length $L=0.5 \mathrm{~m}$ loaded with the distribution of reactances shown in Figure 2.10. 
In conclusion, the application of the Theory of Characteristic Modes together with reactive loading gives the designer a total control over the resonance frequency of a particular mode. However, there is no control over how the rest of modes will be affected by the loading.

Results previously presented show that when a continuous inductive loading is used, the resonance frequency of all modes shifts to lower frequencies, at the same time as its radiating bandwidth worsens. The separation between resonances also diminishes because of the loading, resulting in sharp antiresonances that degrade the impedance bandwidth of the loaded antenna.

\subsubsection{Discrete loading}

The technique presented in section 2.5.1 is not recommendable from a practical point of view, since the physical implementation of the reactive loads shown in Figure 2.10 is really complicated, if not impossible.

An easier solution to modify the resonance frequency of a mode, while minimizing the effect over the rest of modes, consists of using lumped loads at discrete points of the wire dipole. For instance, the resonance frequency of mode $J_{l}$ can be shifted to a lowest frequency by placing an inductive load just at the centre of the dipole, where the mode presents maximum current amplitude (see Figure 2.2). The advantage of this solution is that neither mode $J_{2}$ nor $J_{4}$, will be affected by the loading, since they present a current null at the location of the load. In contrast, mode $J_{3}$, which presents maximum current at the centre of the dipole, will also be altered because of the loading.

The main drawback of this technique is that there is not a systematic procedure to obtain the exact value of the load needed to resonate the mode at the desired frequency, other than cut and try methods. However, if the location of the load coincides with the location of the source, the input impedance values can be used as a reference to obtain an approximated value of the load. To be explicit, in Figure 2.8 it has been demonstrated that the first and second resonances of a centre-fed dipole occur when modes $J_{1}$ and $J_{3}$ resonate, respectively. Then, if a resonance of the input impedance is shifted by placing a lumped load in series with the source, the resonance frequency of the mode responsible for that resonance will be shifted accordingly.

Suppose the objective is to make mode $J_{1}$ resonant at $120 \mathrm{MHz}$. From Figure 2.12 (a) it is extracted that the input impedance of a centre-fed dipole at 120 $\mathrm{MHz}$ is $Z_{\text {in }}=7.7-\mathrm{j} 832 \Omega$. Hence, to obtain a resonance at $120 \mathrm{MHz}$, a reactive load of value $X_{L}=832 \Omega$ should be placed in series with the source. Theoretically, the resonance frequency of mode $J_{l}$ will also be shifted to 120 $\mathrm{MHz}$. 
Figure 2.13 evidences that the input impedance of the centre-fed dipole when loaded with a $832 \Omega$ reactive load at its centre, presents a resonance at $120 \mathrm{MHz}$. With respect to the behaviour of modes, Figure 2.14 shows the variation with frequency of the characteristic angles of the first four modes for this loaded dipole. Due to the loading, mode $J_{1}$ resonates at $120 \mathrm{MHz}$, mode $J_{3}$ at $675 \mathrm{MHz}$, whereas the resonance frequencies of modes $J_{2}$ and $J_{4}$ do not change.

Results for the resonance frequency, radiating bandwidth and quality factor are summarized in Table 2.4. It is worth noting that only the radiating bandwidth of mode $J_{I}$ has been degraded because of the loading. Surprisingly, the radiating bandwidth of mode $J_{3}$ increases in the presence of the load.

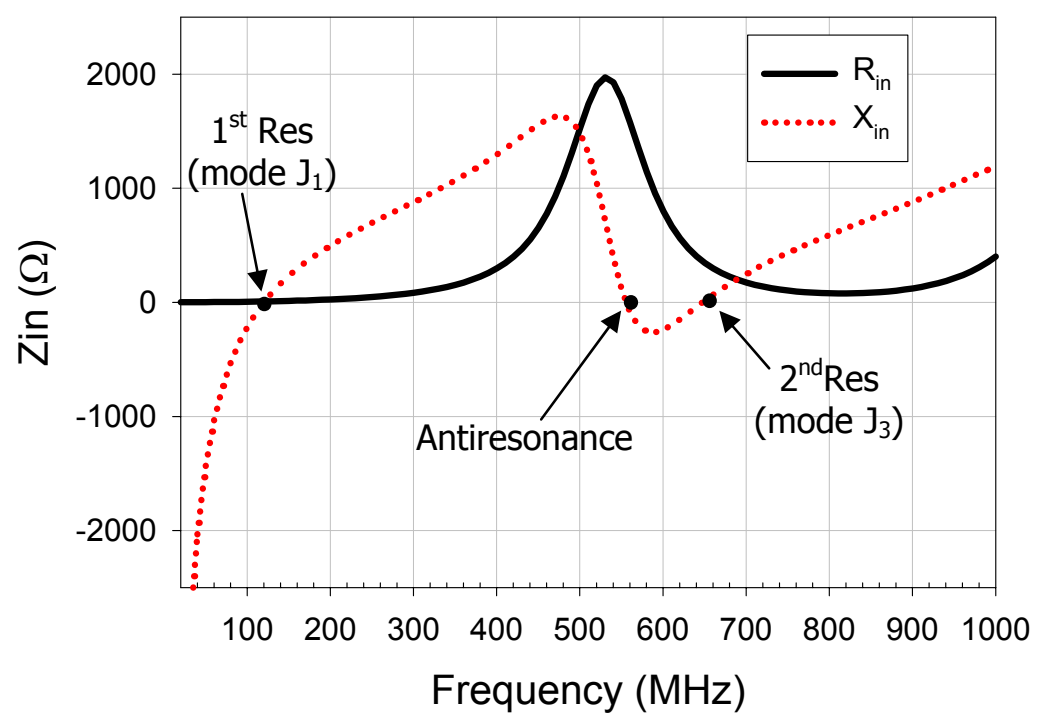

Figure 2.13 Input impedance of the centre-fed dipole when loaded with a 832 $\Omega$ reactive load at its centre.

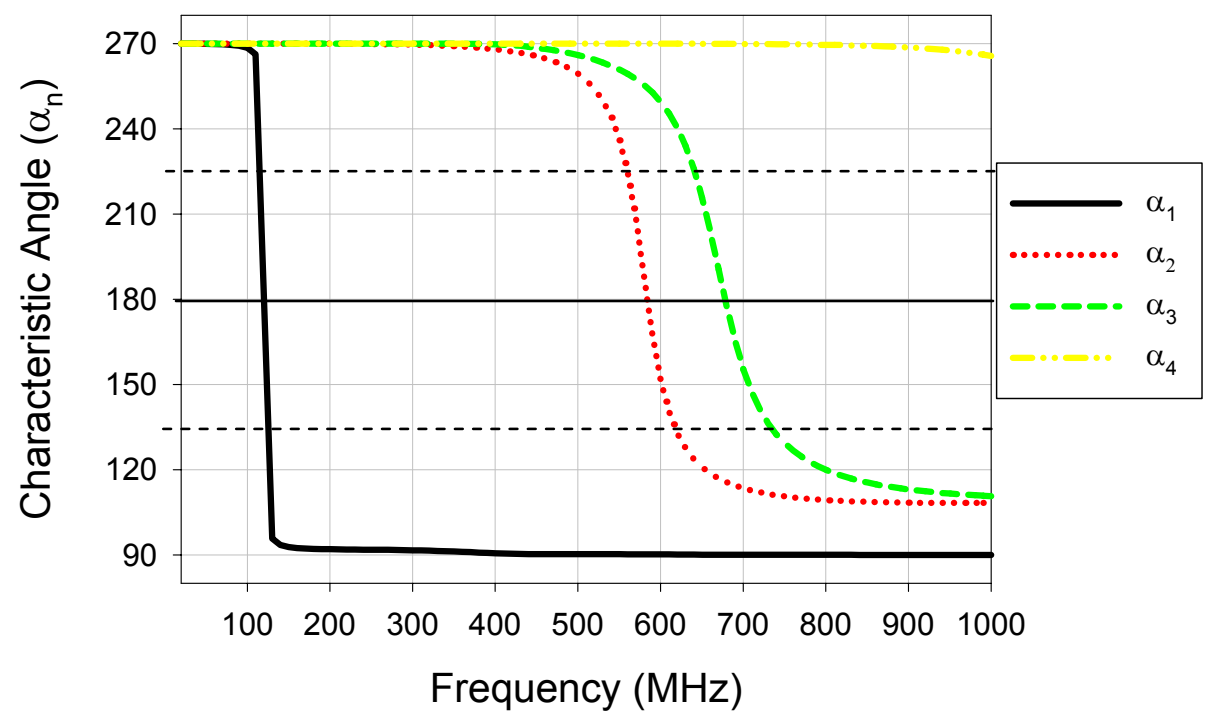

Figure 2.14 Variation with frequency of the characteristic angles for the first four modes of a dipole with a reactive load of $832 \Omega$ placed at its centre. 


\begin{tabular}{|c|c|c|c|c|c|}
\cline { 2 - 6 } \multicolumn{1}{c|}{} & $f_{\text {res }}(\mathrm{MHz})$ & $f_{L}(\mathrm{MHz})$ & $f_{U}(\mathrm{MHz})$ & $B W_{n}(\%)$ & $Q_{\text {rad }, n}$ \\
\hline Mode $J_{1}$ & 120 & 115 & 125 & 8.3 & 12 \\
\hline Mode $J_{2}$ & 590 & 560 & 620 & 10.16 & 9.84 \\
\hline Mode $J_{3}$ & 675 & 640 & 730 & 13.33 & 7.5 \\
\hline
\end{tabular}

Table 2.4. Resonance frequency, radiating bandwidth and quality factor of the first three modes of the dipole with a reactive load of $832 \Omega$ placed at its centre.

From the previous results it outcomes that discrete loading is less harmful with the radiating bandwidth of modes than continuous loading. Nevertheless the current distribution of modes is more affected by discrete loading than by continuous loading, so do radiation patterns. Figure 2.15 depicts the normalized current distribution at first resonance $(120 \mathrm{MHz})$ of the four first modes of the discrete loaded dipole. Observe mode $J_{l}$ exhibits a triangular current distribution that is typical of an elemental dipole. This is because the length of the dipole $(L=0.5 \mathrm{~m})$ is small when compared with the wavelength at $120 \mathrm{MHz}$ $\left(\lambda_{120 \mathrm{MHz}}=2.5 \mathrm{~m}\right)$. For the same reason, the current distribution of mode $J_{3}$ is also nearly triangular close to the location of the load. On the contrary, since modes $J_{2}$ and $J_{4}$ present a current null at the location of the load, their current distributions remain unaltered.

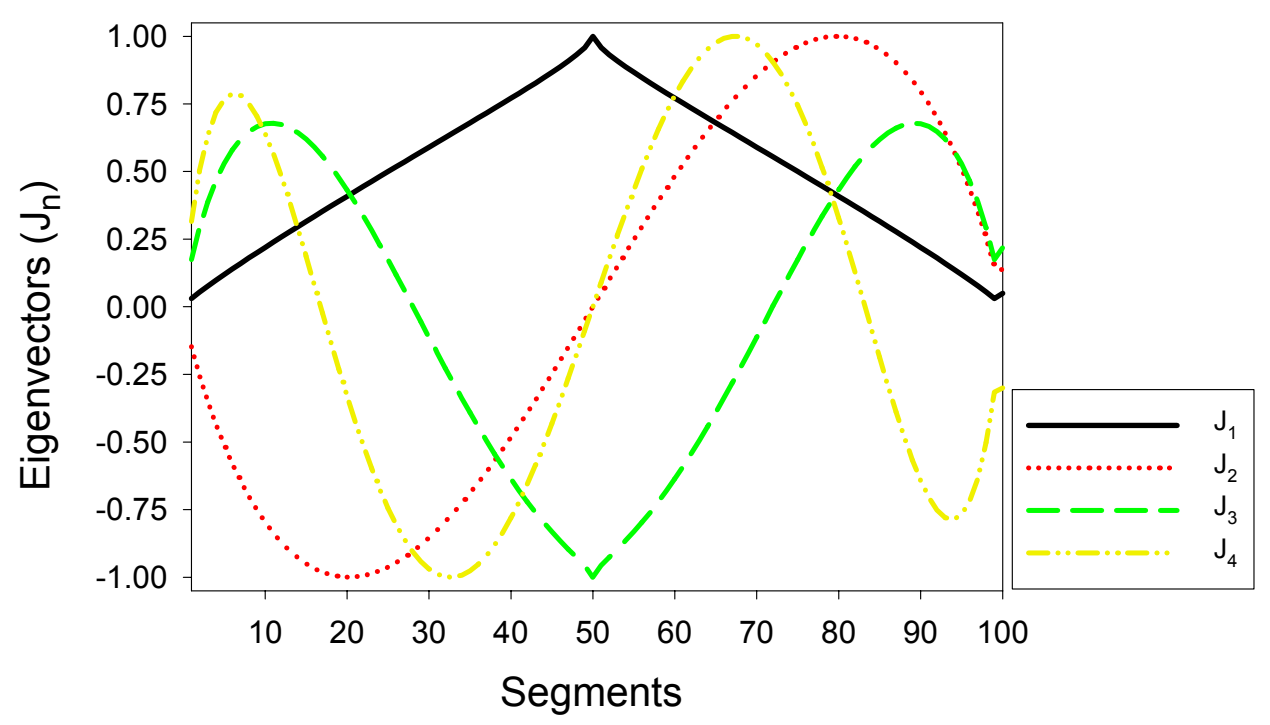

Figure 2.15 Normalized distribution at first resonance ( $f=120 \mathrm{MHz}$ ) for the first four eigencurrents for the dipole with a reactive load of $832 \Omega$ placed at segment 50.

Finally, it should be emphasized that although the procedure described here for determining the value of the reactive load is also valid for resonating higher order modes, results are not as precise as when the fundamental mode is chosen for the shifting. Often, when higher order modes are selected to be reallocated in frequency, the value for the reactive load obtained from the input impedance needs to be slightly modified to achieve the desired shifting. In contrast, the procedure described in section 2.5.1 is always valid and precise whichever the order of the mode is. 


\subsection{Equivalent circuit of an antenna using Characteristic Modes}

A revision of the available literature shows that extensive studies have been reported regarding the derivation of lumped element equivalent circuits for antennas and scatterers [63]-[66]. In special, equivalent circuits for modelling the input impedance of thin-wire structures have long been investigated [67][70].

Actually, although achieving a single frequency circuit model for an antenna is relatively simple, synthesizing a broad-band equivalent circuit is not so easy. Schelkunoff developed a general theory of broad-band equivalent circuits for radiating structures [71], however, he did not address any realizability considerations. Next, other authors proposed a formal equivalent circuit development based on the Singularity Expansion Method (SEM) formalism [72]. This approach is quite difficult as it involves working in the complex frequency plane [73].

Other methods, like the one described in [74], are based on the generation of a rational function approximation with real coefficients in order to model the input impedance of the antenna in the frequency domain. Then, an algorithm, for example a genetic algorithm, can be employed in order to find the best-fit positive-real rational function that is realizable by passive circuits.

However, to some extent all the abovementioned approaches have lacked generality, and realizability issues are not considered.

As shown in section 2.4, the input admittance of an antenna can be expanded in terms of eigenadmittances. Having in mind this input admittance expansion, an equivalent circuit model for the antenna can be synthesized. In this equivalent circuit, modal admittances are modelled by series $R-L-C$ networks. The value of the each individual element of the network is determined from the resonance frequency and the quality factor of the corresponding mode.

To illustrate the method, a broadband equivalent circuit for the centre-fed wire dipole analyzed in section 2.4, is going to be synthesized. This circuit accounts for the excitation of modes $J_{1}$ and $J_{3}$, and is valid from low frequencies to second resonance.

Figure 2.16 shows the proposed lumped element equivalent circuit for the centre-fed dipole. Each mode is modelled by a series $R-L-C$ circuit. The resistance $R_{n}$ accounts for the radiation of the mode. The inductance $L_{n}$, in series with the capacitance $C_{n}$, provides the resonance frequency of the mode

$$
f_{r_{-} n}=\frac{1}{2 \pi \sqrt{L_{n} \cdot C_{n}}}
$$




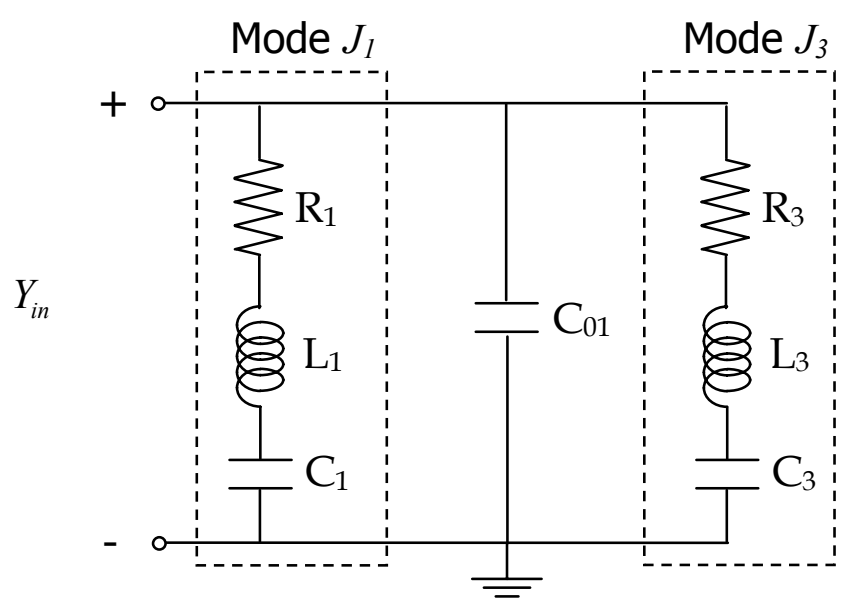

Figure 2.16 Equivalent circuit based on the modal expansion of the input admittance, for the centre-fed wire dipole analyzed in section 2.4.

The procedure for obtaining the value of each element of the circuit begins with the determination of the resistances $R_{1}$ and $R_{3}$. When the mode is at resonance, its associated modal admittance is pure reactive

$$
\left.Y_{n}\right|_{f=f_{r-n}}=\frac{1}{R_{n}}
$$

From Figure 2.9 (a) it can be extracted that at resonance frequency $\left(f_{r I}=290\right.$ $\mathrm{MHz}$ ), the modal admittance of mode $J_{1}$ is $Y_{1}=13 \mathrm{~m}^{-1}$. This value yields a reactance $R_{1}=77 \Omega$. Likewise, for the case of mode $J_{3}$, the modal admittance at resonance $\left(f_{r 3}=890 \mathrm{MHz}\right)$ is $Y_{3}=9.5 \mathrm{~m}^{-1}$, which results in $R_{3}=105 \Omega$.

Next, the inductances $L_{1}$ and $L_{3}$ are obtained using the modal quality factors calculated in section 2.3.1. The quality factor of the series $R-L-C$ circuit representing each mode is

$$
Q_{r a d, n}=\frac{\omega_{r_{-} n} \cdot L_{n}}{R_{n}}
$$

When the quality factor in (2.38) takes the value $Q_{\text {rad, },}=7.25$, and $Q_{\text {rad }, 3}=12.72$, the inductances $L_{1}=306.4 \mathrm{nH}$ and $L_{3}=238.8 \mathrm{nH}$ are obtained, respectively.

Once the inductances of the circuit have been calculated, the capacitances $C_{l}$ and $C_{3}$ can be determined using (2.36). The corresponding values are $C_{1}=0.98 \mathrm{pF}$ and $C_{3}=0.134 \mathrm{pF}$. 
Finally, a capacitance $C_{01}$ is placed in parallel with the $R-L-C$ circuits. This capacitance interacts with the series resonant circuits, and facilitates the modelling of the antiresonance (parallel resonance) that occurs at $525 \mathrm{MHz}$. If higher order modes had been considered in the circuit, this capacitance $C_{01}$ would not have been needed.

The input admittance of the equivalent circuit of Figure 2.16 is

$$
Y_{i n}=Y_{1}+j \omega C_{0}+Y_{3}=\frac{1}{R_{1}+j \omega L_{1}+\frac{1}{j \omega C_{1}}}+j \omega C_{0}+\frac{1}{R_{3}+j \omega L_{3}+\frac{1}{j \omega C_{3}}}
$$

Substituting the already known elements in expression (2.39) it is obtained that at the antiresonance

$$
\left.Y_{i n}\right|_{f=525 \mathrm{MHz}}=2.027 \cdot 10^{-4}-j 7.34 \cdot 10^{-4}+j \omega C_{0}
$$

Then, the value of the parallel capacitance is derived by enforcing the imaginary part of (2.40) to be zero

$$
C_{0}=0.22 p F
$$

Figure 2.17 compares the actual input admittance of the centre-fed dipole computed with method of moments, with that provided by the equivalent circuit proposed in Figure 2.16. As can be seen both results agree favourably.

Lastly, it should be pointed out that a lumped element equivalent circuit for an antenna can be very useful, especially if the antenna needs to be integrated in a system, and modelled together with other microwave components, using specific commercial software, such as Microwave Office.

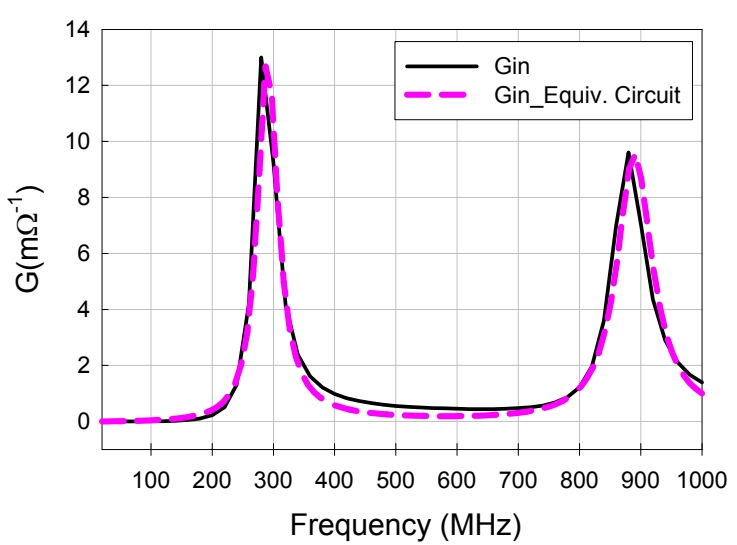

(a)

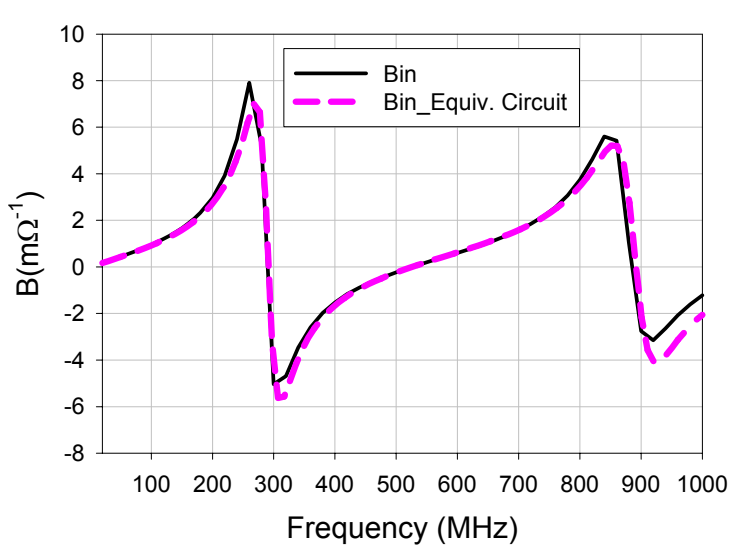

(b)

Figure 2.17 Comparison of the input admittance of the centre fed dipole computed with MoM, with the input admittance obtained for the equivalent circuit shown in Figure 2.16. 


\subsection{Introduction}

In Chapter 2 a review of the Theory of Characteristic Modes was presented using a straight wire dipole as an example. The present chapter is devoted to the modal analysis of wire loops of different geometry. Wire loops are particularly interesting since special non-resonant modes, which are dominant at lowest frequencies, appear on them. The dependency of the degeneracy of modes with the symmetry of the loop will be studied in detail. Drawing an analogy with the folded dipole, modes will be classified in antenna modes and transmission line modes. Finally, more complex wire structures will be analyzed in order to demonstrate that the Theory of Characteristic Modes can be applied to conducting wire objects of arbitrary shape.

There exist some publications calculating the natural frequencies and modal current distributions of natural modes [75]-[76]. All these studies are based on the SEM approach [72], and work in the complex frequency domain. However, none of the literature has shown so far a systematic modal analysis of loop antennas based on the Theory of Characteristic Modes, which provides a clearer physical insight than the SEM approach.

\subsection{Special non-resonant modes and degenerated modes}

Let us begin analyzing the behaviour of the special non-resonant modes that are inherent in closed wire structures. For this purpose, a circular wire loop is going to be considered.

Circular loops are classical wire antennas that have been extensively studied over the last six decades for UHF applications. A search through the available literature shows that if the loop is small with respect to the wavelength of operation, the current distribution on it will be uniform [77]-[78]. Otherwise, for electrically large loops a sinusoidal current distribution is assumed [79]-[80].

The circular loop that is going to be used for this study is depicted in Figure 3.1. The loop is placed at the $X Y$ plane, and it presents diameter $d=0.229 \mathrm{~m}$, and wire radius $r=0.5 \mathrm{~mm}$. The wire is assumed to be a perfect conductor of no conducting loss. The perimeter of the loop is divided in 50 segments, as 50 triangular functions have been used for expansion and testing in the computation of the impedance matrix. The current distribution at first resonance $(f=440 \mathrm{MHz})$ for the first five eigencurrents of this loop is shown in Figure 3.2. According to their current distribution, modes $J_{0}, J_{2}$ and $J_{4}$ present even symmetry, and modes $J_{1}$ and $J_{3}$ present odd symmetry. 
For the sake of clarity, Figure 3.3 provides schemes of the projection of the eigencurrents along the loop perimeter. Note segments are numbered counter clockwise beginning from $\mathrm{Y}$ axis. The first two modes, $J_{1}$ and $J_{2}$, present two current nulls at $\phi=90^{\circ}$ and at $\phi=0^{\circ}$, respectively. Modes $J_{3}$ and $J_{4}$ are higher order modes with four current nulls. Nulls of mode $J_{3}$ are at $\phi=90^{\circ}$ and $\phi=0^{\circ}$, whereas nulls of mode $J_{4}$ are at $\phi= \pm 45^{\circ}$.

Complementarily with these results, Figure 3.4 describes the variation with frequency of the characteristic angles associated to these first five current modes of the circular loop.

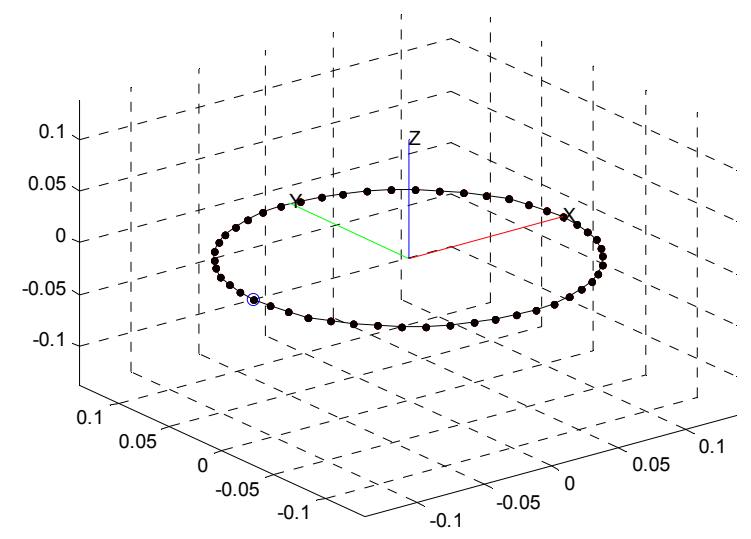

Figure 3.1 Circular wire loop of diameter $d=0.229 \mathrm{~m}$ and wire radius $r=0.5 \mathrm{~mm}$.
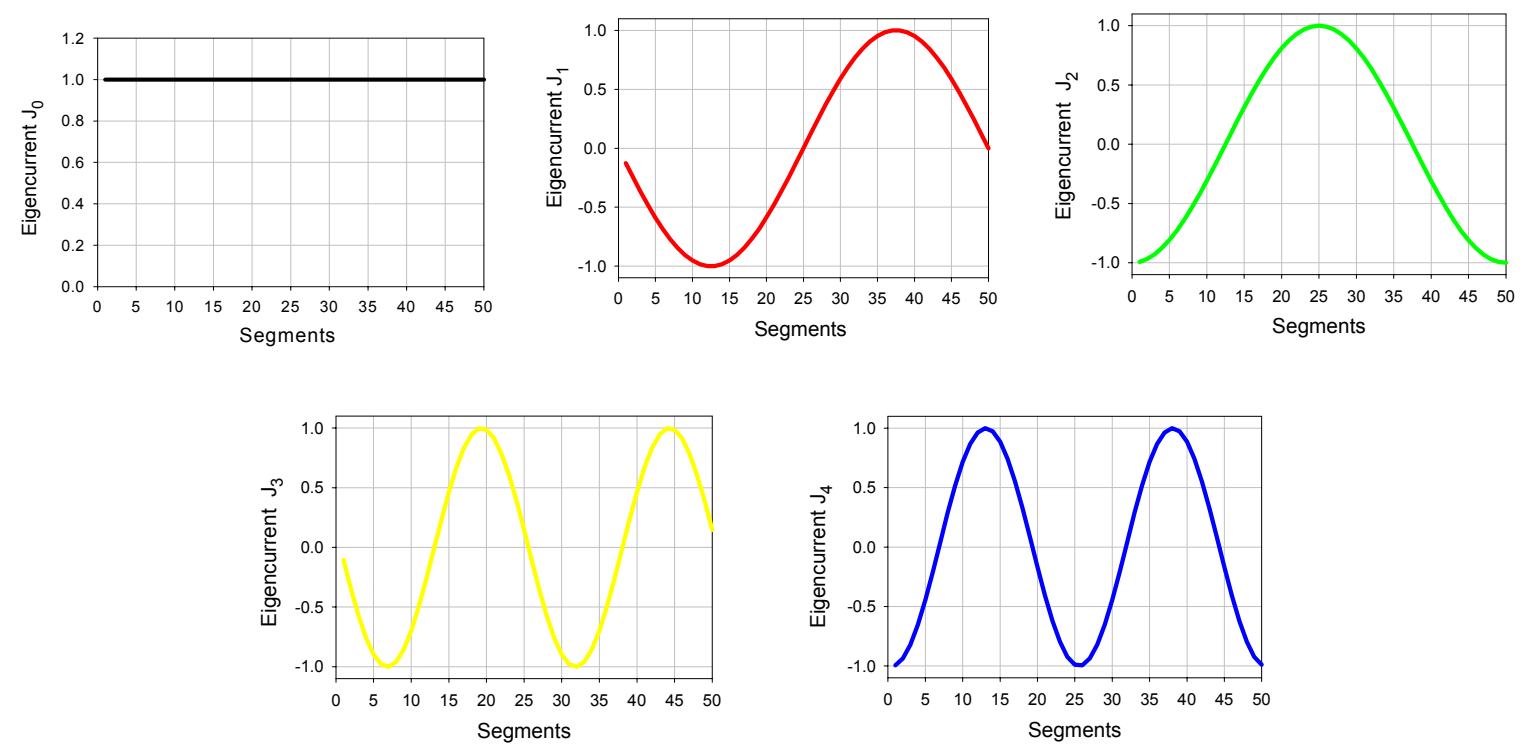

Figure 3.2 Normalized current distribution at first resonance ( $f=440 \mathrm{MHz}$ ) of the first five eigencurrents $J_{n}$ of the circular loop shown in Figure 3.1. 


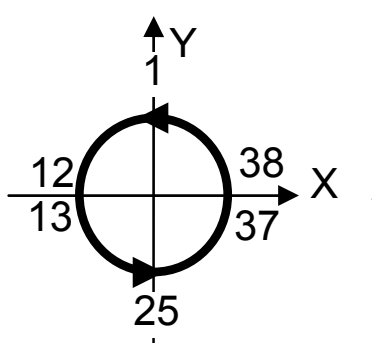

Mode $\mathrm{J}_{0}$

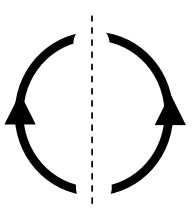

Mode $\mathrm{J}_{1}$

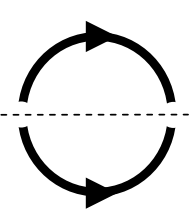

Mode $\mathrm{J}_{2}$
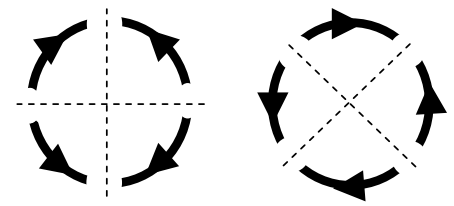

Mode $\mathrm{J}_{3}$

Mode $\mathrm{J}_{4}$

Figure 3.3 Schemes of the projection of eigencurrents $J_{n}$ along the circular loop perimeter that is divided in fifty segments.

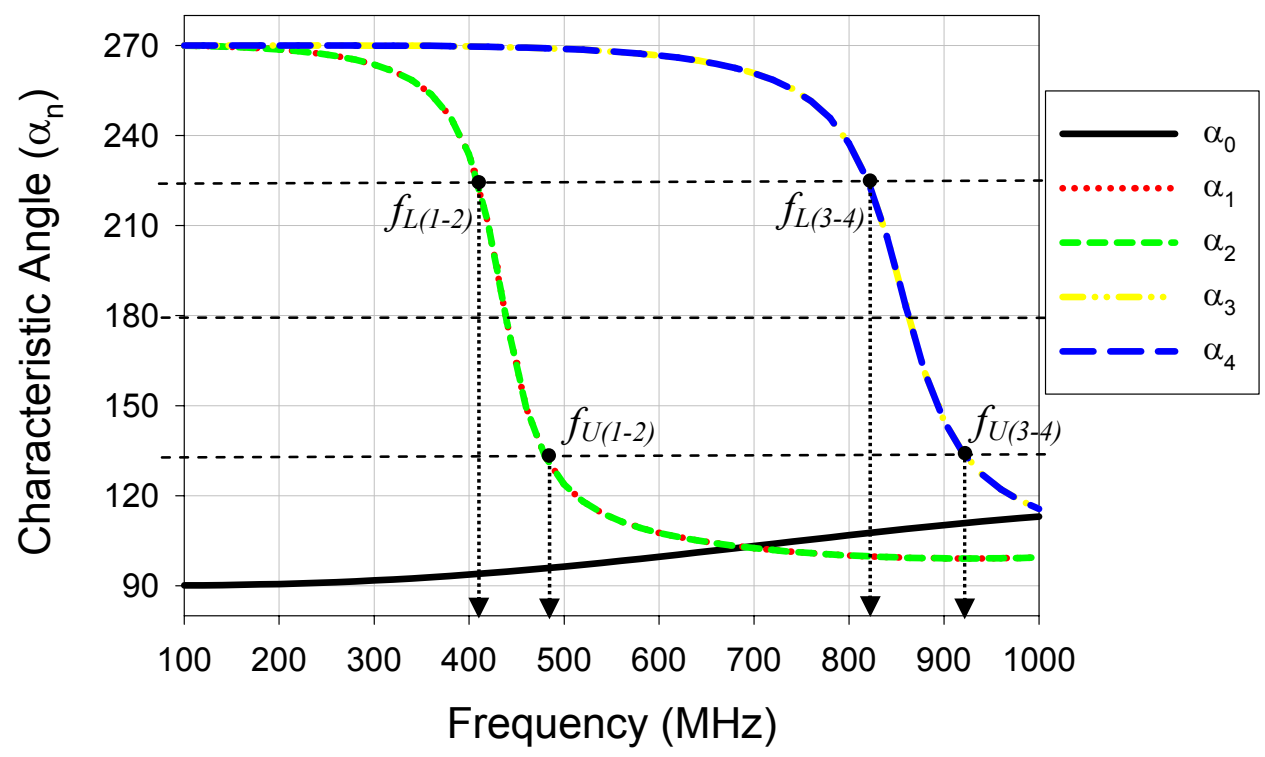

Figure 3.4 Variation with frequency of the characteristic angle $\alpha_{n}$ associated to the eigencurrents of the circular loop shown in Figure 3.2.

Relevant information can be obtained from the previous figures. On one hand, Figures 3.2 and 3.3 evidence that mode $J_{0}$ presents uniform current forming a closed loop along the perimeter of the loop. This mode is responsible for the typical uniform current distribution that appears in electrically small loops. Figure 3.4 proves that this mode exhibits special behaviour when compared with the rest of modes, as its characteristic angle stays below $180^{\circ}$ at every frequency. This means that mode $J_{0}$ does not resonate and it always contribute to store magnetic energy. On the other hand, due to the revolution symmetry of the loop, modes resonate in pairs, except for the special mode $J_{0}$. In the studied frequency range, there are two pairs of degenerated modes with identical resonance frequency, $J_{1}-J_{2}$, and $J_{3}-J_{4}$. 
As stated by Figure 3.4, degenerated modes present the same characteristic angle at every frequency. First pair of degenerated modes resonates at 440 $\mathrm{MHz}$, when the perimeter of the loop is approximately $\lambda$. Second pair resonates at $860 \mathrm{MHz}$, when the perimeter of the loop is $2 \lambda$. Furthermore, degenerated modes present exactly the same current distribution, but with $90^{\circ}$ phase difference, for the case of modes $J_{1}$ and $J_{2}$, and with $45^{\circ}$ phase difference, for the case of modes $J_{3}$ and $J_{4}$.

It should be pointed out that the current distributions of the degenerated pairs shown in Figure 3.2 are perfect sinusoidal functions. The current distribution of modes $J_{1}$ and $J_{2}$, corresponds with a sinusoidal functions of one cycle, whereas the current distribution of modes $J_{3}$ and $J_{4}$, is that of a two cycles sinusoidal function.

Additionally, the radiating bandwidth of modes can be determined using the information provided by the characteristic angle curves shown in Figure 3.4. As it was demonstrated in Chapter 2, the characteristic angle values that correspond with one-half the power radiated at resonance, are $135^{\circ}$ and $225^{\circ}$. Hence, the upper and lower frequencies, $f_{U}$ and $f_{L}$, which appear in equation (2.24), are the frequencies at which characteristic angles are $135^{\circ}$ and $225^{\circ}$, respectively.

Table 3.1 sums up the radiating bandwidth and quality factor of the modes of the circular loop. These results have been obtained using equations (2.24) and (2.25), together with the information provided by Figure 3.4. In next section, the values of Table 3.1 will be compared with similar ones obtained for the modes of other wire loops of different geometry.

\begin{tabular}{|c|c|c|c|c|c|}
\cline { 2 - 6 } \multicolumn{1}{c|}{} & $f_{\text {res }}(\mathrm{MHz})$ & $f_{L}(\mathrm{MHz})$ & $f_{U}(\mathrm{MHz})$ & $B W_{n}(\%)$ & $Q_{\text {rad }, n}$ \\
\hline Modes $J_{1}$ and $J_{2}$ & 440 & 410 & 475 & 14.77 & 6.77 \\
\hline Modes $J_{3}$ and $J_{4}$ & 860 & 820 & 920 & 11.63 & 8.60 \\
\hline
\end{tabular}

Table 3.1. Resonance frequency, radiating bandwidth and quality factor for the first two pairs of degenerated modes of the circular loop shown in Figure 3.1.

Figure 3.5 shows the azimuthal radiation pattern $\left(\theta=90^{\circ}\right)$ at $440 \mathrm{MHz}$ of the modal electric fields $E_{n}$ produced by the current modes $J_{n}$ of Figure 3.2. It can be observed that the radiation pattern generated by mode $J_{0}$ presents nearly omni directional characteristic. The radiation patterns created by degenerated modes are identical but with a phase rotation of $90^{\circ}$ for the first pair $\left(J_{1}\right.$ and $\left.J_{2}\right)$, and a phase rotation of $45^{\circ}$ for the second pair $\left(J_{3}\right.$ and $\left.J_{4}\right)$. Observe also that the number of lobes increases with the order of the mode, or what is the same, in correspondence with the number of current nulls of the mode. 

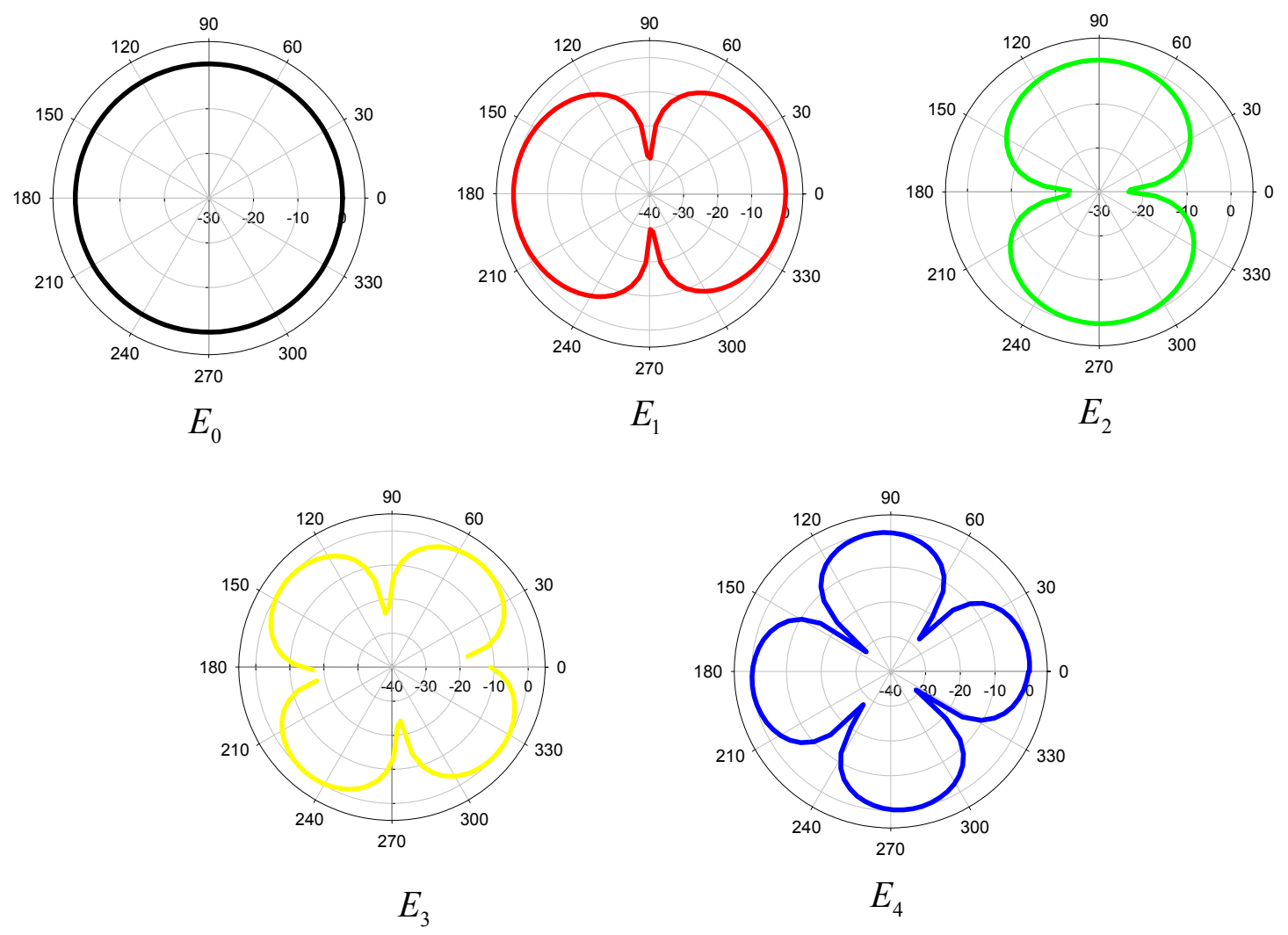

Figure 3.5 Azimuthal radiation pattern $\left(\theta=90^{\circ}\right)$ at $440 \mathrm{MHz}$ of the modal electric fields $E_{n}$ produced by the current modes $J_{n}$ of Figure 3.2.

No mention has yet been made of the excitation of modes. Due to the rotational symmetry of the loop, when a single source is used anywhere along the loop perimeter, only one mode of each degenerated pair is excited. This assertion can be verified by means of Figure 3.6 which studies the contribution of modal admittances $\left(Y_{n}\right)$ to the total input admittance of the circular wire loop $\left(Y_{i n}\right)$, when a delta gap source is placed at segment 25.

In Figure 3.6 it is observed that because of the location of the source, only modes with even symmetry, $J_{0}, J_{2}$ and $J_{4}$ are excited. The uniform current mode $J_{0}$ dominates at lowest frequencies. Mode $J_{2}$ prevails from $300 \mathrm{MHz}$ to $600 \mathrm{MHz}$, and it causes the resonance at $440 \mathrm{MHz}$. Mode $J_{4}$ governs the antenna behaviour from $800 \mathrm{MHz}$ to $1000 \mathrm{MHz}$, and it is responsible for the second resonance at $860 \mathrm{MHz}$. Conversely, antiresonances are not caused by a single mode, but by the combination of several of them. For instance, the first antiresonance that occurs at $200 \mathrm{MHz}$, when the loop perimeter is $0.5 \lambda$, results from the combination of the inductive mode $J_{0}$ and the capacitive mode $J_{2}$. Likewise, the second antiresonance at $600 \mathrm{MHz}$, which corresponds with a loop perimeter of $1.5 \lambda$, is the result of the interaction of modes $J_{0}, J_{2}$ and $J_{4}$. 


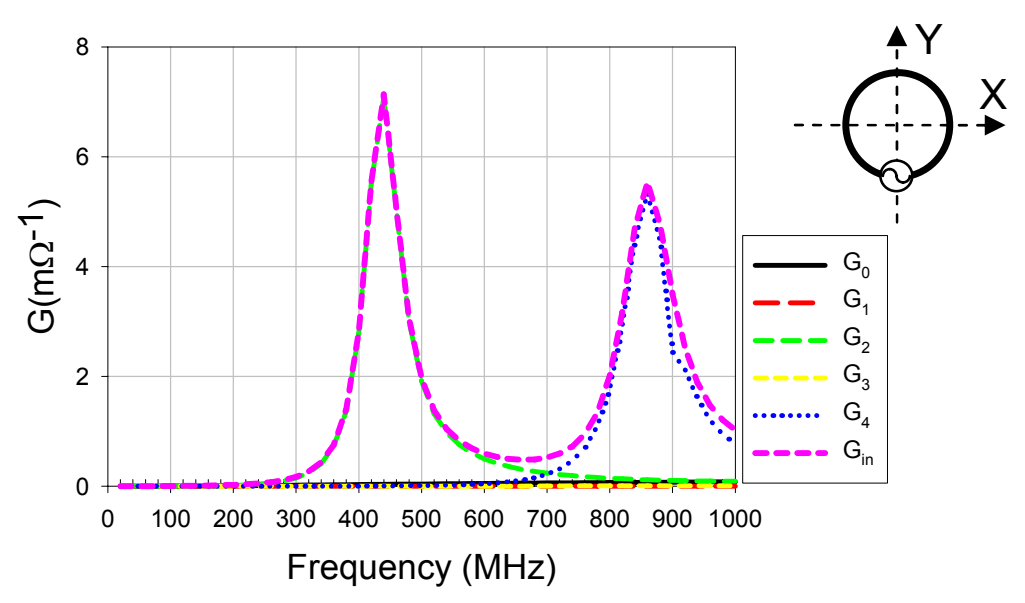

(a) Real part

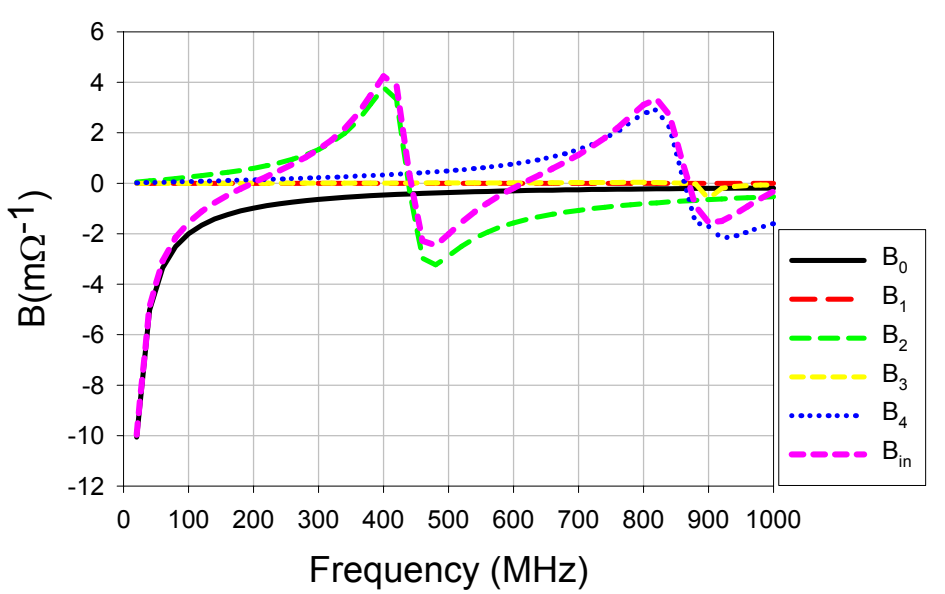

(b) Imaginary part

Figure 3.6 Contribution of modal admittances $Y_{n}$ to the total input admittance $Y_{\text {in }}$ of the circular loop shown in Figure 3.1.

The representation of the modal admittances in Figure 3.6 helps to find out which modes are excited when the source is placed at segment 25 , however, by means of modal admittances it is difficult to determine if the excited modes are well matched to the source. In order to establish the input bandwidth of modes, a modal input voltage standing wave ratio $\left(V S W R_{n}\right)$ can be calculated. Figure 3.7 shows the VSWR of modes $J_{2}$ and $J_{4}$, which has been obtained from the modal admittances of Figure 3.6. The total VSWR of the antenna has also been plotted in Figure 3.7. Observe that although the total VSWR of the antenna can not be expressed as a linear combination of the $V S W R$ of the excited modes, when they are represented together it is easy to recognize which modes are contributing to the total input bandwidth of the antenna at each frequency. For the circular loop under study, there are two operating bands, one at $450 \mathrm{MHz}$ and the other at $850 \mathrm{MHz}$. Both bands present poor impedance matching, so the input bandwidth of the antenna is not very good. First band can be clearly attributed to mode $J_{2}$, and second band is due to the excitation of mode $J_{4}$. 
It is interesting to note that because of the revolution symmetry of the loop, if the delta gap source were located at segment 1, results obtained in Figure 3.6 and Figure 3.7 would be exactly the same. On the contrary, if the feeding were positioned at segments 12.5 or 37.5 , modes $J_{1}$ and $J_{4}$ would be excited, while the total input admittance would behave exactly the same way as in Figure 3.6, and the total $V S W R$ would also remain unchanged.

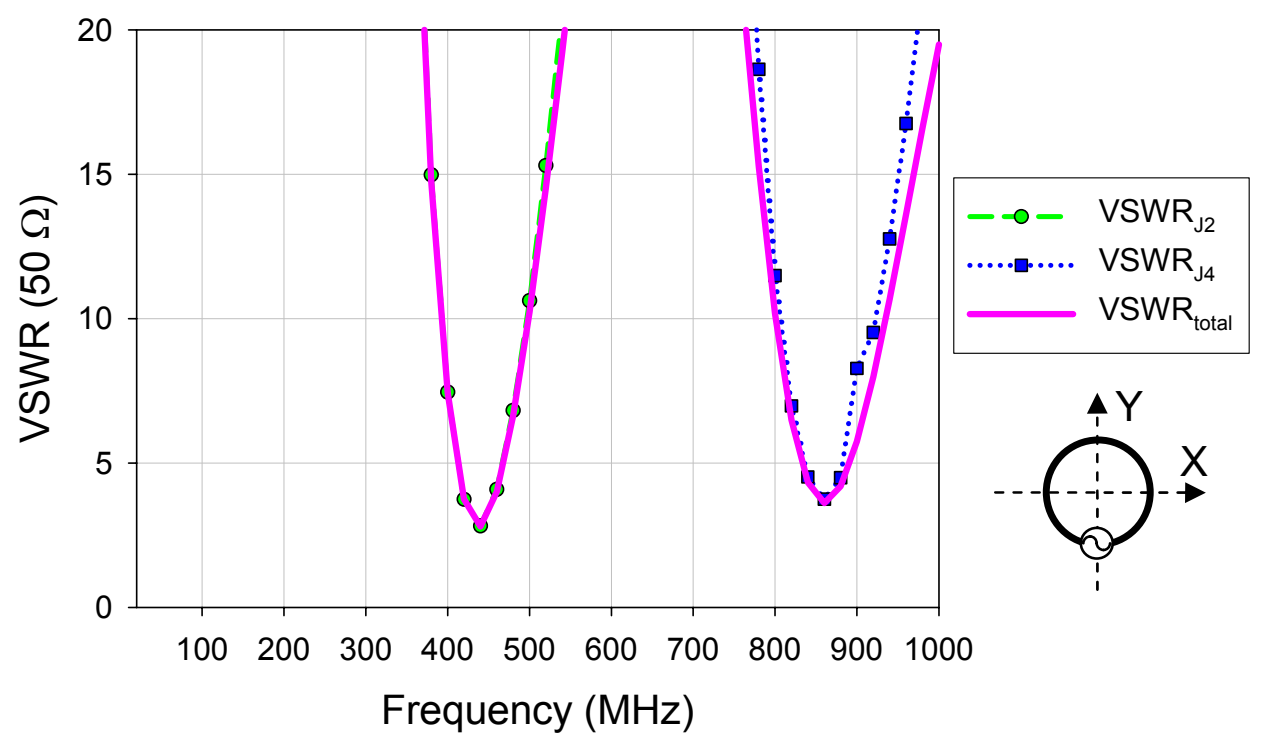

Figure 3.7 Contribution of the VSWR of modes $J_{2}$ and $J_{4}$ to total $V S W R$ of the circular loop antenna when it is fed at segment 25.

Finally, if both modes of a pair of degenerated modes want to be excited simultaneously, for example to obtain circular polarization, the rotational symmetry of the loop should be broken by introducing some kind of asymmetry. This asymmetry can be a discrete load, a stub, or an open gap at any position of the loop. Another alternative to excite simultaneously two degenerated modes consist in using two excitation sources in quadrature. However, as the number of excited modes increases, also does the number of antiresonances. Remember that antiresonances are due to the transition from one mode to other, and they produce high resistance values that affect remarkably the broad-band property of the antenna. Because of this negative effect of antiresonances, when an antenna presents a wideband radiating mode it is desirable to avoid the excitation of higher order modes that would interact with it, generating an antiresonance. In general, the further two consecutive resonances are, the softer the resultant antiresonace is.

Next section explains how to use reactive loading to break the rotational symmetry and the degeneracy pattern of the modes of the circular wire loop, in order to obtain circular polarization. 


\subsection{Use of reactive loading for obtaining circular polarization}

In section 2.5 of Chapter 2 it was explained how to use reactive loading in combination with the Theory of Characteristic Modes for increasing the electric length of a wire dipole while maintaining its physical length. In this section, discrete reactive loading is going to be employed for splitting the first pair of degenerated modes of the circular loop, with the aim of obtaining circular polarization using a single feeding source.

It is a well-known fact that to obtain circular polarization it is necessary to combine two orthogonal and linearly polarized modes, with the same current amplitude and with a phase difference of $90^{\circ}$. As commented above, a circular loop with a single source positioned anywhere along the perimeter, yields linear polarization, as only one mode of the each degenerated pair is excited. By inserting reactive loads at different positions along the loop perimeter, degeneracy of modes is broken and circular polarization can be obtained using a single feeding source at the precise point. This is achieved just combining two of the previous degenerated modes, for example modes $J_{1}$ and $J_{2}$. The main advantage of this method is that no balun circuit or quadrature hybrid is needed to excite the two orthogonal modes. To illustrate this concept the circular loop of section 3.2 is going to be used again.

Suppose two reactive loads are placed at segments 1 and 25 of the circular loop (see Figure 3.3 to remember how the segments were numbered). These two loads will shift the resonance frequency of modes with even symmetry $\left(J_{2}\right.$ and $\left.J_{4}\right)$ to lowest frequencies. In contrast, modes that present odd symmetry $\left(J_{1}\right.$ and $\left.J_{3}\right)$, and hence current nulls at segments 1 and 25 , will not be altered by the loading. Next, if a feeding source is placed at a position $45^{\circ}$ away from one of the loads, for example at segment 6 , where neither mode $J_{1}$ nor $J_{2}$ have current nulls, and where both of them present the same current amplitude, circular polarization will be obtained at the frequency at which the two modes combine appropriately.

After some testing, two identical inductive loads of $130 \Omega$ positioned at segments 1 and 25, have been found to be the optimum choice for obtaining the desired shifting between modes $J_{1}$ and $J_{2}$. Figure 3.8 shows the modal significance curves $\left(M S_{n}\right)$ of these two modes for the loaded circular loop. It can be observed that at $409 \mathrm{MHz}$, both modes present exactly the same normalized current amplitude. Moreover, as shown in Figure 3.9, which depicts characteristic angle curves for modes $J_{1}$ and $J_{2}$, both modes are in phase quadrature at $409 \mathrm{MHz}$. If a delta gap source is now placed at segment 6, circular polarization will be obtained at $409 \mathrm{MHz}$, because at this frequency, there are two orthogonal modes that match the required conditions.

Figure 3.10 shows the elevation radiation pattern $\left(\phi=0^{\circ}\right)$ at $409 \mathrm{MHz}$ of the modal fields created by modes $J_{1}$ and $J_{2}$. It can be observed that both modes are linearly polarized and that their dominant components ( $E_{1 \phi}$ for mode $J_{1}$ and $E_{2 \theta}$ for $J_{2}$ ) are orthogonal. 


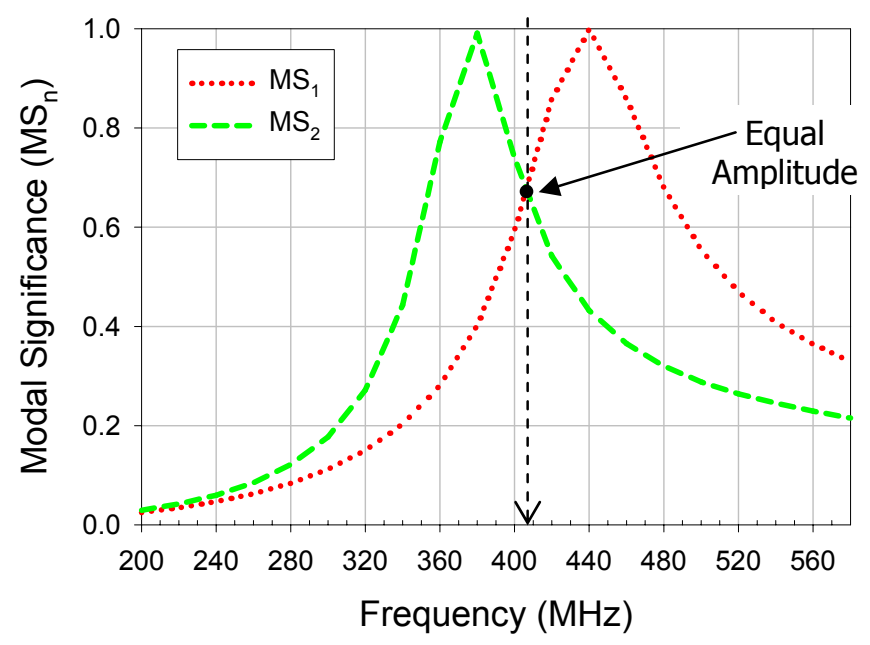

Figure 3.8 Modal significance curves $\left(M S_{n}\right)$ for the first two modes of the loaded circular loop.

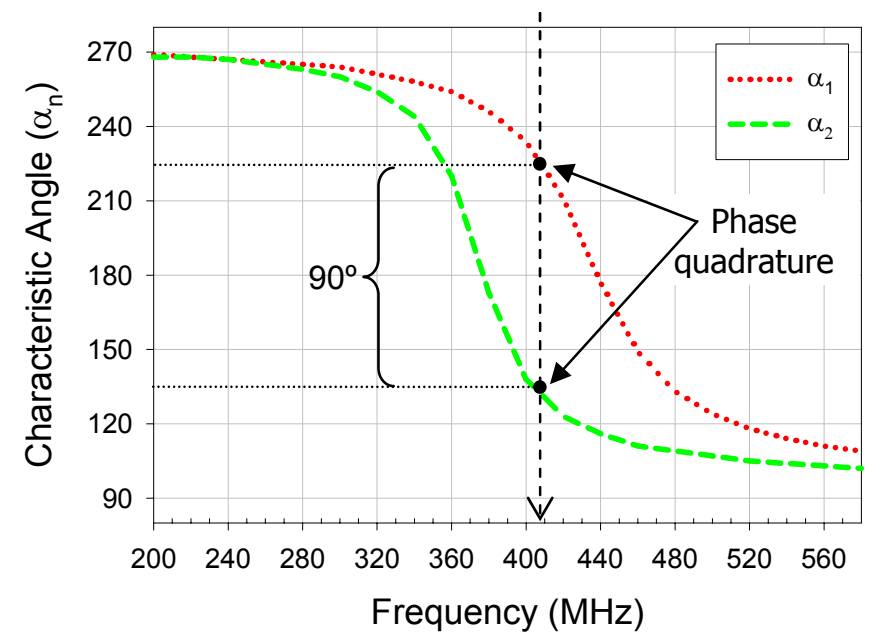

Figure 3.9 Characteristic angle curves $\left(\alpha_{n}\right)$ for the first two modes of the loaded circular loop.

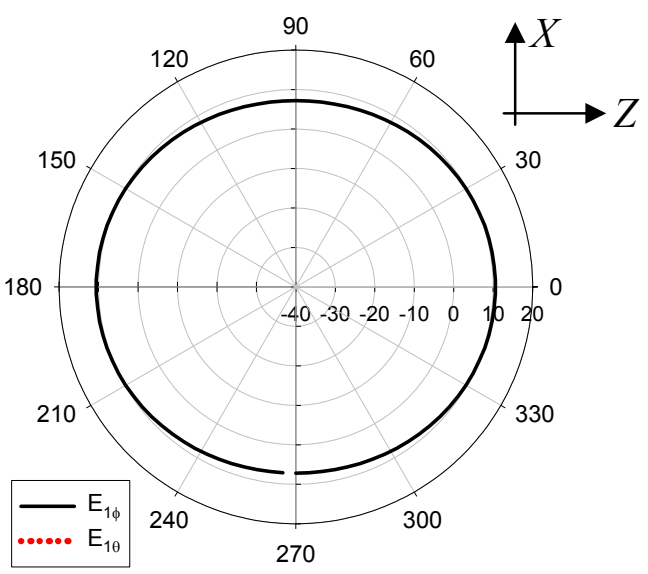

(a) Mode $\mathrm{J}_{1}$

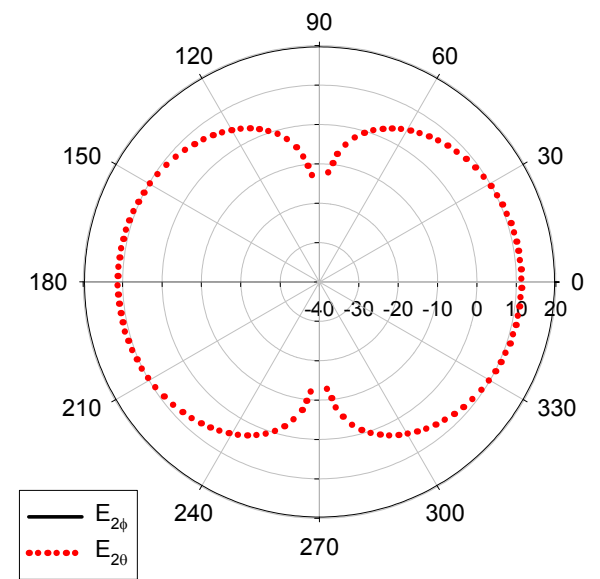

(b) Mode $\mathrm{J}_{2}$

Figure 3.10 Elevation radiation pattern $\left(\phi=0^{\circ}\right)$ at $409 \mathrm{MHz}$ of the modal electric fields produced by modes $J_{1}$ and $J_{2}$ of the loaded circular loop. 
Using the modal field components of modes $J_{1}$ and $J_{2}$ shown in Figure 3.10, an axial ratio at broadside direction $\left(\theta=0^{\circ}\right)$ can be defined as

$$
\left.A R\right|_{\phi=0, \theta=0}=\left.\frac{E_{2, \theta}}{E_{1, \phi}}\right|_{\phi=0, \theta=0}
$$

The magnitude of the field due to mode $J_{0}$, which will also be excited if the source is placed at segment 6 , is very small, so it has been neglected in equation (3.1).

At last, Figure 3.11, which depicts the axial ratio computed using (3.1), attests that the circular loop is circularly polarized at $409 \mathrm{MHz}$, at broadside direction $\left(\theta=0^{\circ}\right)$.

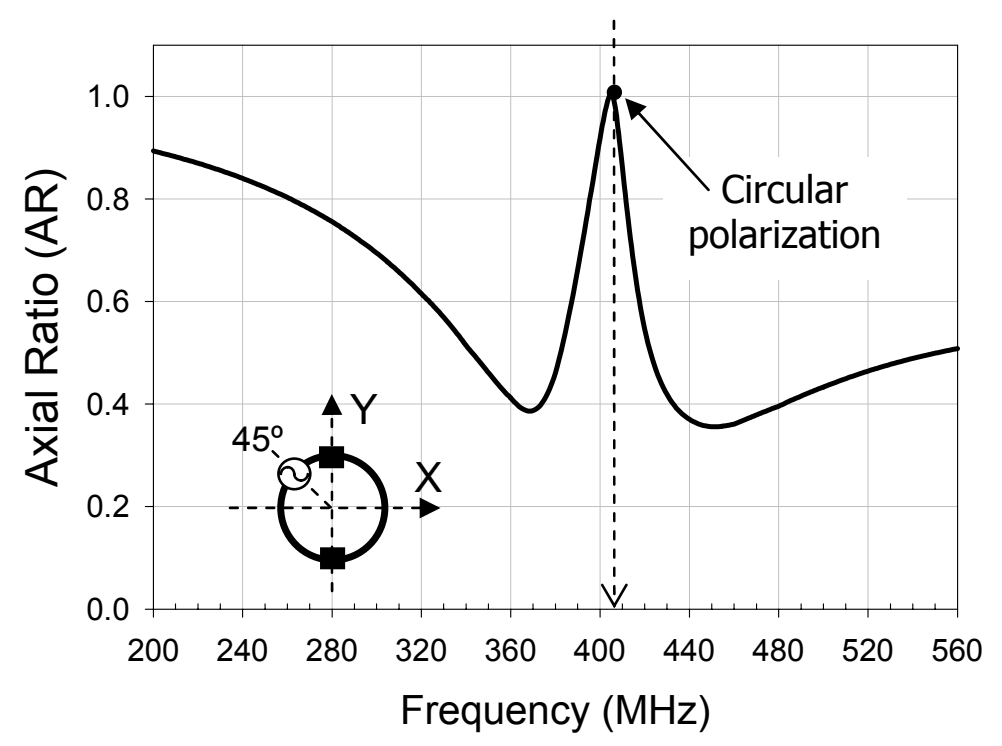

Figure 3.11 Axial ratio at broadside direction $\left(\theta=0^{\circ}\right)$ obtained for the loaded circular loop when driven at segment 6.

Note that circular polarization could have also been obtained by placing a single inductive load of $260 \Omega$ at segment 1 , however, this solution produces elliptically polarized modal fields that unbalance and degrade the axial ratio.

Similar approaches to the one described here, to obtain a circularly polarized circular loop have been recently published [81]-[82]. The main difference with the present approach is that in [82], the reactive load required is achieved by introducing a small gap in the wire loop in spite of using a lumped element.

From the example just presented, it is extracted that modifying the rotational symmetry of the circular loop, pairs of degenerated modes split. Next section analyzes in detail the effect of the symmetry of different wire loops on the degeneracy pattern of its modes. 


\subsection{Effect of the symmetry of loops on the degeneracy of modes}

It is a well-known fact that the current distribution on a wire loop antenna depends upon its shape and size. The most typical loop shapes being used for wireless communications are the circular, square and triangular loop. Square loop and triangular loop antennas have been applied to the HF and UHF bands due to its easy of construction and its broad-band input bandwidth. However, the circular loop antenna is usually preferred for the UHF band because of its higher directivity gain.

As reported in [76], for regular polygonal loop antennas, the degeneracy pattern of modes depends on the number of sides of the loop. This conclusion has been reached by analyzing the natural frequencies and modal current distributions of natural modes, obtained when applying SEM approach for canonical loop structures.

Once a modal characterization of the circular loop has been performed, let us go one step further, and analyze other loop geometries, such as the square loop, or the triangular loop, in order to demonstrate that the degeneracy pattern of modes is related directly to the symmetry of the loop rather than to the number of sides.

\subsubsection{Modal analysis of the square loop antenna.}

Figure 3.12 sketches the geometry of the square loop antenna that is going to be analyzed in first place. The loop is located at the $X Y$ plane, and its square dimension is $L=0.229 \mathrm{~m}$. The wire radius used for the simulation is $r=0.5 \mathrm{~mm}$, and the wire is considered a perfect conductor.

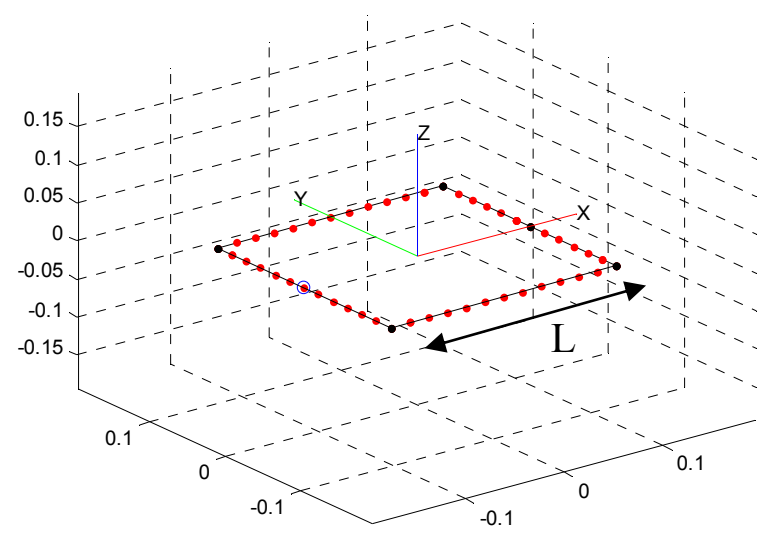

Figure 3.12 Square wire loop of dimension $L=0.229 \mathrm{~m}$ and wire radius $r=0.5$ $\mathrm{mm}$. 
Figure 3.13 shows the normalized current distribution at first resonance $(f=360 \mathrm{MHz})$ of the first modes of this square loop antenna. For this case, 48 triangular functions have been used for expansion and testing. Once more, schemes of the projection of the eigencurrents along the loop perimeter are provided in Figure 3.14.

First remarkable aspect of Figure 3.13 is that although the current distributions of modes resemble quite a lot those of the circular loop, they are not perfect sinusoidal functions any longer. Second important issue is that now the current distribution of mode $J_{0}$ is not uniform, since it decreases at the corners of the loop (segments 6,18, 30 and 42). Observe also that modes $J_{1}$ and $J_{2}$ present two current nulls, modes $J_{3}$ and $J_{4}$ four current nulls, and modes $J_{5}$ and $J_{6}$ six current nulls.
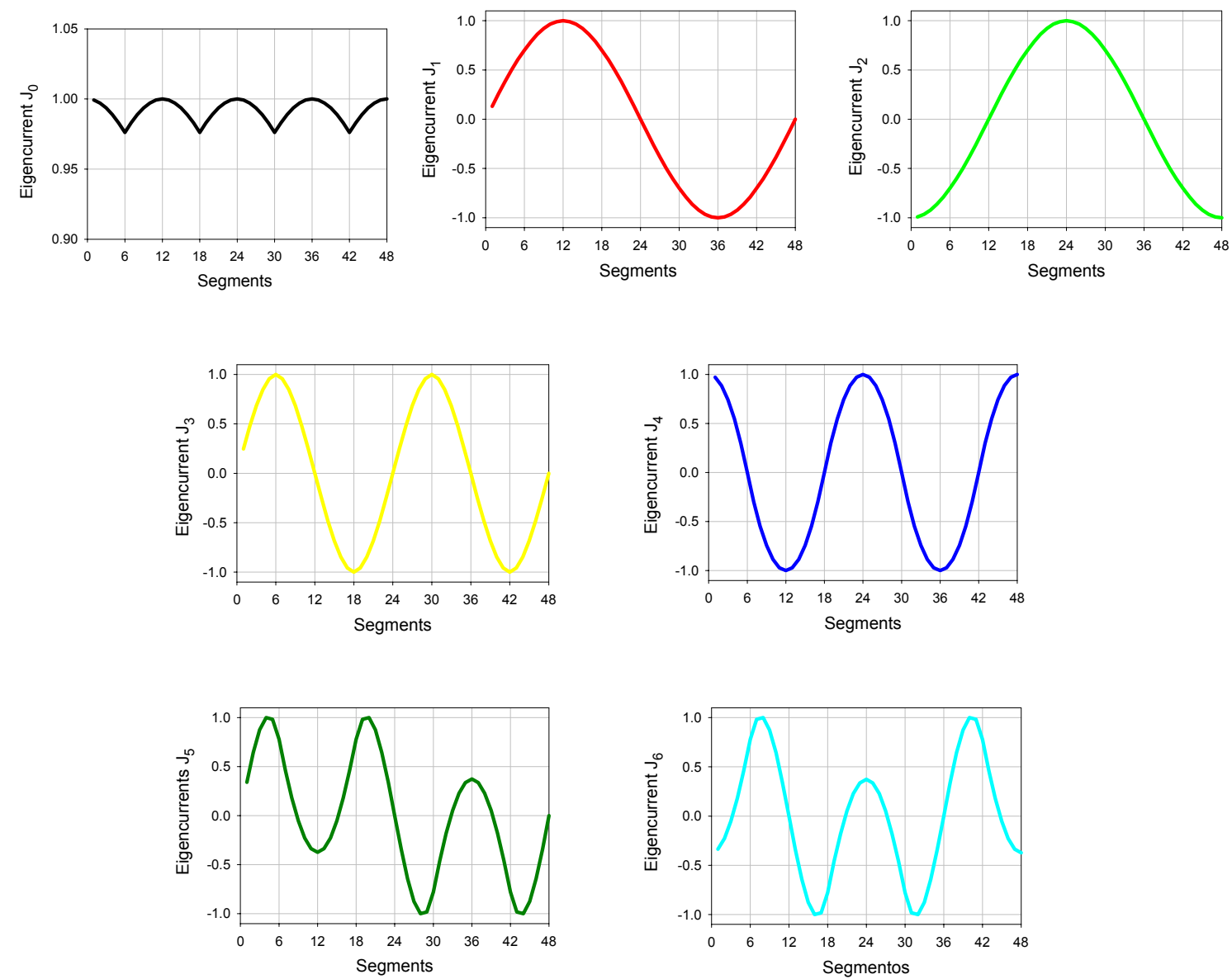

Figure 3.13 Normalized current distribution at first resonance $(f=360 \mathrm{MHz})$ of the first eigencurrents of the square loop shown in Figure 3.12. 


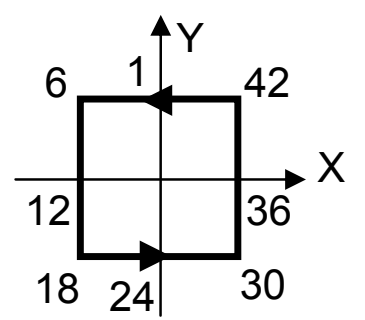

Mode $J_{0}$

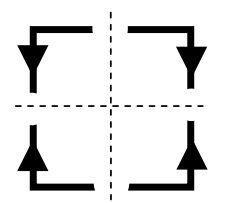

Mode $J_{3}$

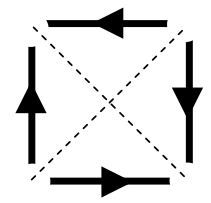

Mode $J_{4}$

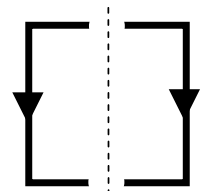

Mode $J_{1}$

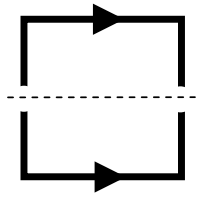

Mode $J_{2}$

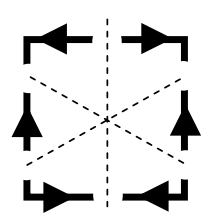

Mode $J_{5}$

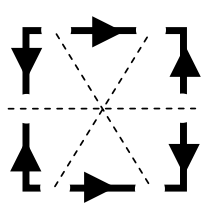

Mode $J_{6}$

Figure 3.14 Schemes of the projection of eigencurrents $J_{n}$ of Figure 3.13 along the perimeter of the square loop.

As in the analysis of the circular loop, next step is to determine the resonance frequency and radiating bandwidth of the modes, using the information given by the characteristic angles. From Figure 3.15 it is inferred that in the studied frequency range there are only two pairs of degenerated modes, $J_{1}-J_{2}$ and $J_{5}-J_{6}$. It is important to highlight that modes $J_{3}$ and $J_{4}$, which are associated to the second harmonic frequency, are not degenerated since their associated characteristic angles do not behave the same way. This is due to the fact that the square loop does not present rotational symmetry. The square loop is only symmetric with respect to $X$ axis and $Y$ axis, so when the current distribution of mode $J_{3}$ in Figure 3.14 is rotated $45^{\circ}$, it does not correspond with the current distribution of mode $J_{4}$, and the degeneracy of these two modes disappears.

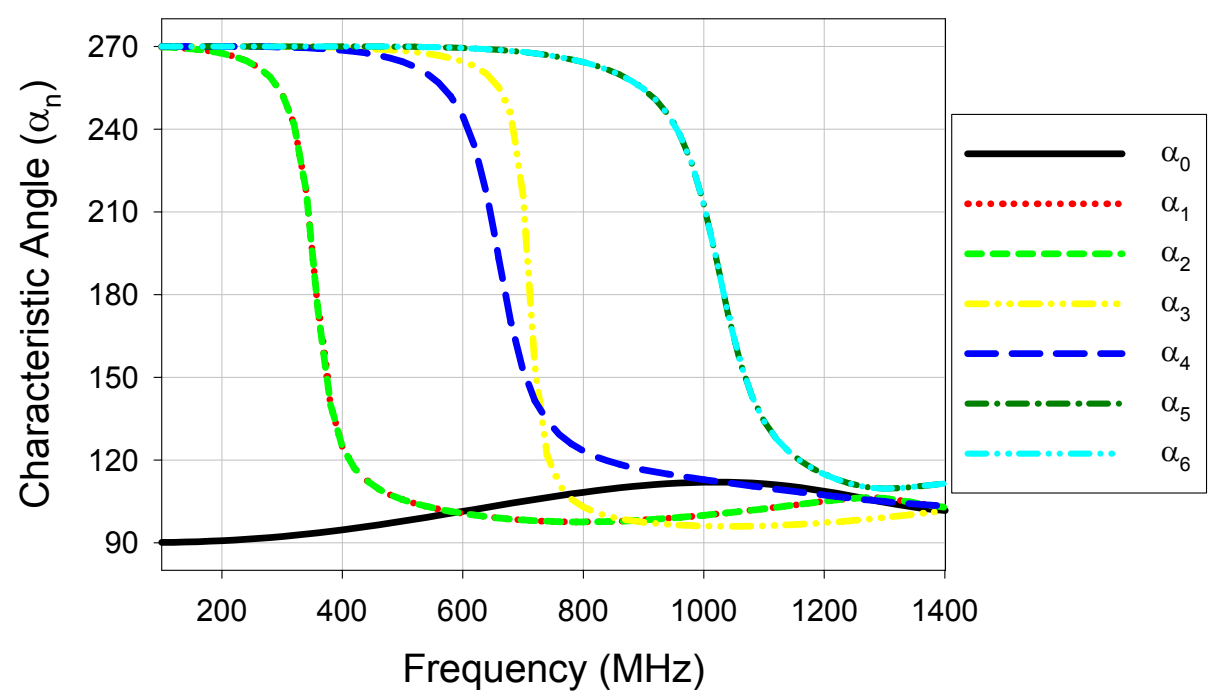

Figure 3.15 Variation with frequency of the characteristic angle $\alpha_{n}$ associated to the eigencurrents of the square loop shown in Figure 3.12. 
Table 3.2 summarizes the resonance frequency, radiating bandwidth and quality factor associated to the current modes of the square loop. Results in Table 3.2 show that the bandwidth associated to the modes of the square loop is wider than that obtained for the modes of a circular wire loop occupying the same area. It can also be observed that mode $J_{4}$ exhibits wider bandwidth than the rest of modes.

\begin{tabular}{|c|c|c|c|c|c|}
\cline { 2 - 6 } \multicolumn{1}{c|}{} & $f_{\text {res }}(\mathrm{MHz})$ & $f_{L}(\mathrm{MHz})$ & $f_{U}(\mathrm{MHz})$ & $B W_{n}(\%)$ & $Q_{\text {rad }, n}$ \\
\hline Modes $J_{l}$ and $J_{2}$ & 360 & 330 & 400 & 19.44 & 5.14 \\
\hline Mode $J_{3}$ & 710 & 690 & 730 & 5.63 & 17.75 \\
\hline Mode $J_{4}$ & 670 & 630 & 780 & 22.39 & 4.46 \\
\hline Modes $J_{5}$ and $J_{6}$ & 1030 & 980 & 1100 & 11.65 & 8.58 \\
\hline
\end{tabular}

Table 3.2. Resonance frequency, radiating bandwidth and quality factor for the first resonant modes of the square loop shown in Figure 3.12.

The wideband behaviour of mode $J_{4}$ can be explained analyzing Figure 3.13 and Figure 3.14. Observe that mode $J_{4}$ has current nulls at the corners of the loop, and current maxima at the centre of the loop arms. Thus, this mode can be interpreted as a combination of two vertical and two horizontal straight dipoles. Just the opposite, mode $J_{3}$ presents current nulls at the centre of the loop arms, and maxima at the corners, so it can be understood as four square dipoles. The effective length and radiation efficiency of a square dipole are smaller than these of a straight dipole. In fact, as shown in Figure 3.16, the current distribution of mode $J_{3}$ is equivalent to four diagonal dipoles that form a smaller square loop rotated $45^{\circ}$. Because of this reduction of the effective length, mode $J_{3}$ resonates at a higher frequency than mode $J_{4}$.

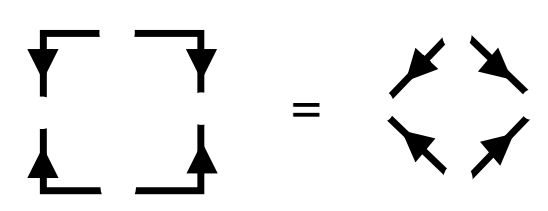

Figure 3.16 Equivalence between the current distribution of mode $J_{3}$ and four diagonal dipoles forming a smaller square loop rotated $45^{\circ}$.

Hence, studying the current distribution of modes it seems evident that mode $J_{4}$ presents considerable wider radiating bandwidth than mode $J_{3}$, since its current distribution can be considered that of four straight dipoles forming a square. However, the wideband behaviour of mode $J_{4}$ could have also been explained by the corner effect [83]-[84]. 
The corner effect is a theory that discusses the relation between the current distribution and the corners of a curved wire antenna. This theory was especially developed for polygonal loop antennas, and it states that when a current null in the standing wave distribution hits a corner of a loop, the loop has a current pattern of very low standing wave ratio, and in turn, broadband impedance characteristic.

The problem is that existing publications regarding the corner effect are focused on analyzing the relation between the source location and the input bandwidth for different polygonal loop antennas, but they do not give a physical justification of why the input bandwidth increases.

Obviously, the Theory of Characteristic Modes yields a more consistent explanation of the bandwidth increase than the corner effect, by interpreting the current distributions of the modes of polygonal loops as arrangements of dipoles, and by analyzing the effective length of these dipoles.

Since the current distribution of modes in Figure 3.14 can be considered as an array of straight and square dipoles (two dipoles for the case of modes $J_{I}$ and $J_{2}$, four for modes $J_{3}$ and $J_{4}$, and six for modes $J_{5}$ and $J_{6}$ ), the azimuthal radiation pattern due the modal electric fields generated by these current modes should be that of the corresponding array of dipoles. This is confirmed by Figure 3.17 which depicts the azimuthal radiation patterns $\left(\theta=90^{\circ}\right)$ at $360 \mathrm{MHz}$ associated to the current modes of Figure 3.13.

With regard to the excitation of modes, results presented above suggest that mode $J_{4}$ should be excited if broad input bandwidth is to be achieved at a square loop antenna. To excite mode $J_{4}$, a delta gap source may be placed at the centre of one of the loop arms, for example at segment 24, where this mode presents maximum current.

Figure 3.18 compares the total VSWR of the square loop antenna when fed at segment 24, with the modal VSWR obtained for the first seven modes of the square loop. As in the circular loop example, modal VSWR have been computed from modal admittances. It is observed that for this particular location of the source, only modes $J_{2}, J_{4}$ and $J_{6}$ are excited. Each mode is responsible for an operation band of the antenna. As observed, although mode $J_{4}$ has been demonstrated to be quite an efficient radiating mode, in this particular case, it is not well matched to the excitation, so the resulting operating band at $670 \mathrm{MHz}$ is not really wide.

Due to the symmetry of the loop, similar results to that presented in Figure 3.18 could had been obtained placing the excitation source either at segments 1,12 or 36 . However, if the excitation is located at one of the vertex of the loop results are a bit different. 
Figure 3.19 depicts the total VSWR obtained for the square loop when it is fed at one vertex (segment 18), and the corresponding VSWR of modes. Results are similar to those obtained in Figure 3.18. The main difference with Figure 3.18 is that now mode $J_{3}$ is excited instead of $J_{4}$. In addition, degenerated modes $J_{1}-J_{2}$ and $J_{3}-J_{4}$ are excited simultaneously. As a result of the excitation of mode $J_{3}$, the second operating band is slightly shifted to higher frequencies, and although this band is narrower than the one provided by mode $J_{4}$ in Figure 3.18, it presents better matching at resonance frequency.

Further results related with the square loop antenna driven at one or more corners can be found in [85].
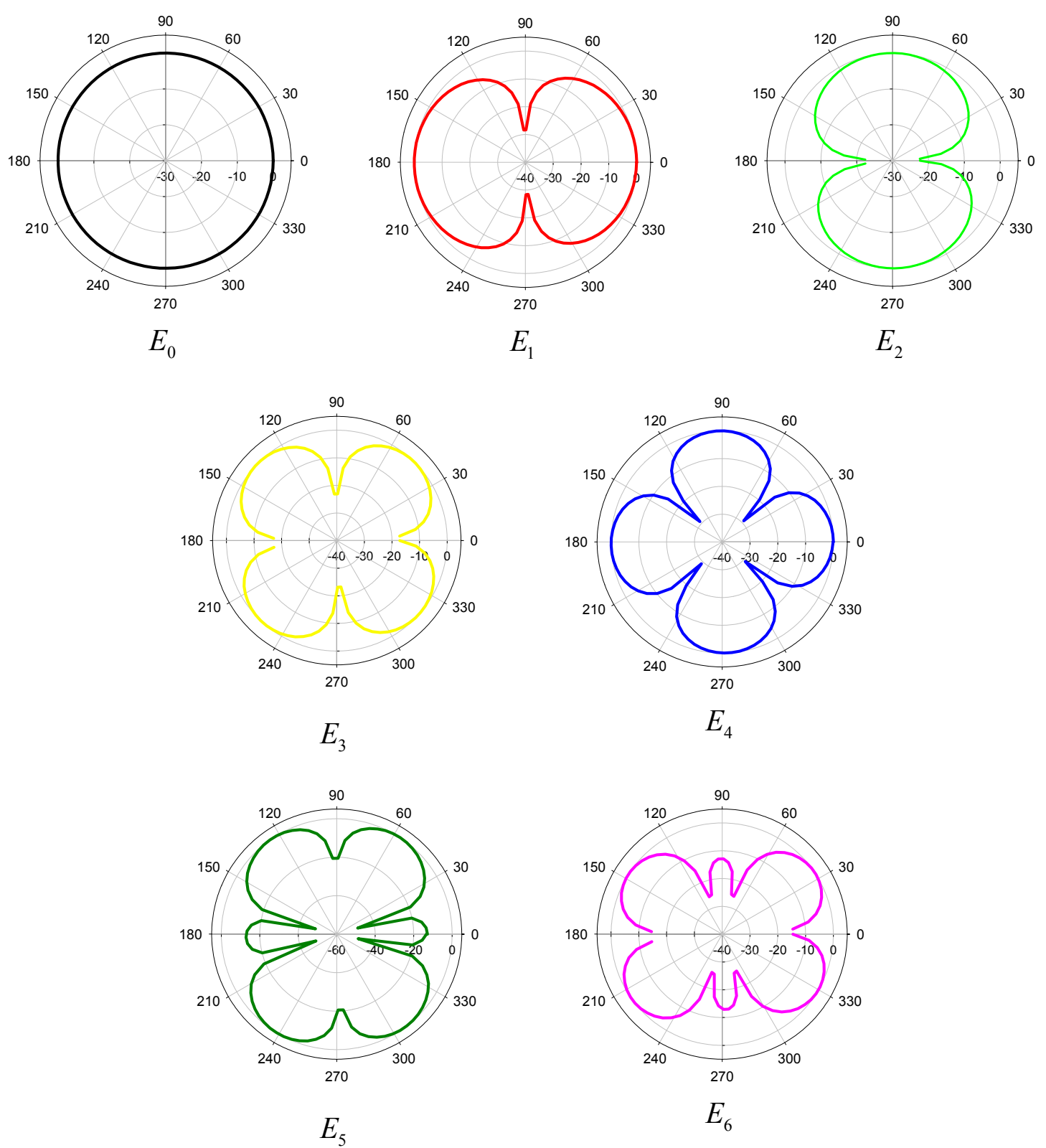

Figure 3.17 Azimuthal radiation pattern $\left(\theta=90^{\circ}\right)$ at $360 \mathrm{MHz}$ of the modal electric fields $E_{n}$ produced by the current modes $J_{n}$ of Figure 3.13. 


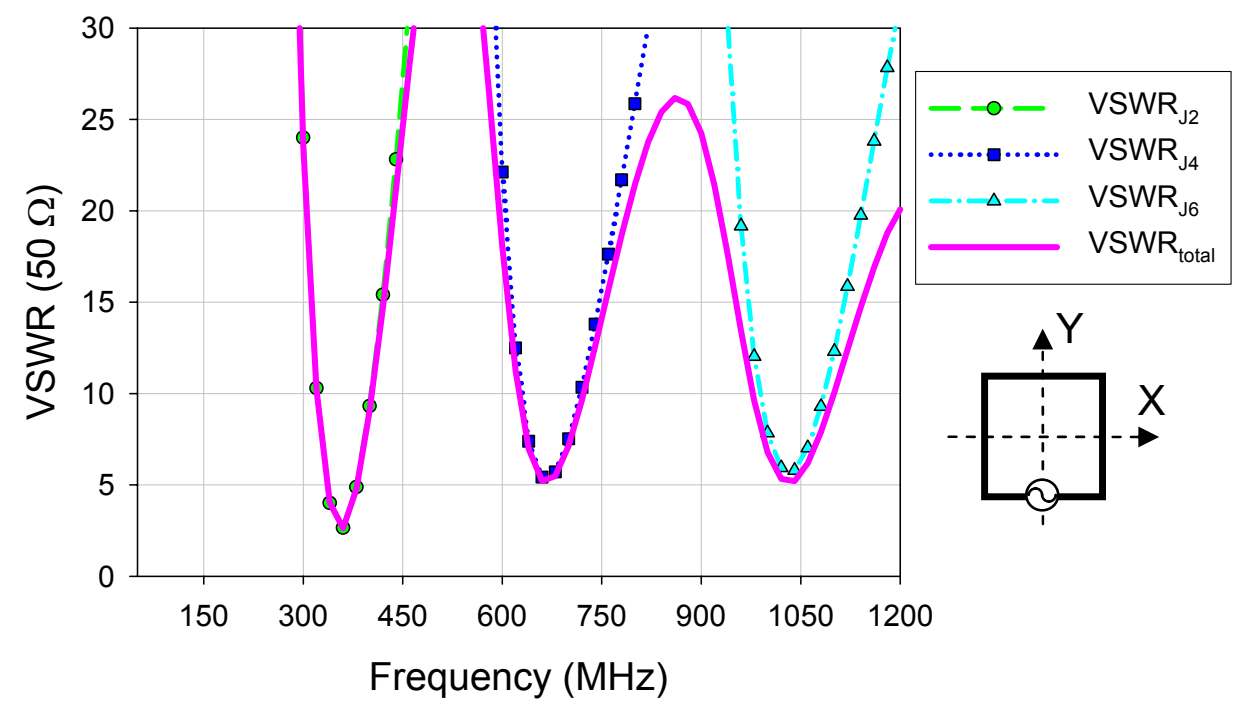

Figure 3.18 Contribution of the $V S W R$ of modes $\mathrm{J}_{2}, \mathrm{~J}_{4}$ and $\mathrm{J}_{6}$, to total $V S W R$ of the square loop antenna when it is fed at segment 24.

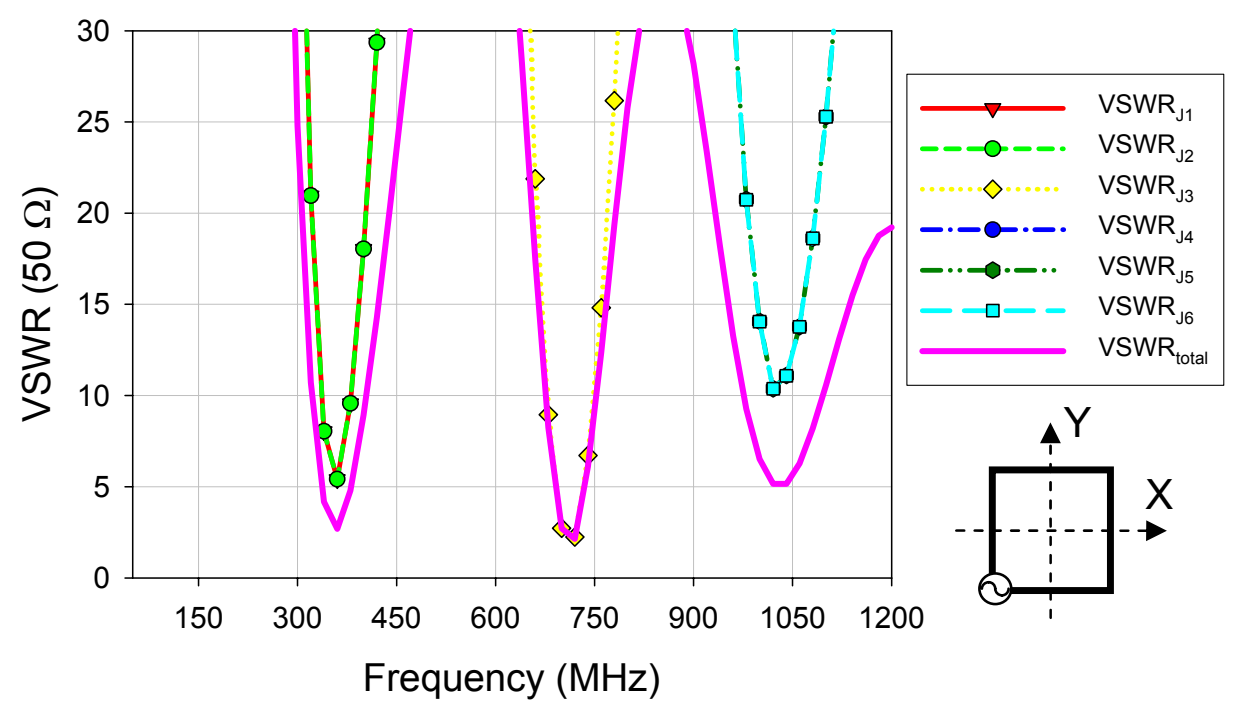

Figure 3.19 Contribution of the VSWR of modes to total VSWR of the square loop antenna when it is fed at segment 18. 


\subsubsection{Modal analysis of a regular triangular loop antenna.}

As explained before, the symmetry of a loop antenna plays a crucial role in the determination of the degeneracy of modes. This section deals with the modal analysis of the regular triangular loop antenna shown in Figure 3.20, which is symmetric with respect to $X$ axis. This loop of arm length $L=0.229 \mathrm{~m}$ is located at the $X Y$ plane. A perfect conducting wire of radius $r=0.5 \mathrm{~mm}$ has been used for its simulation. The loop perimeter is divided in 36 segments, since this is the number of basis functions that has been considered for the numerical calculations.

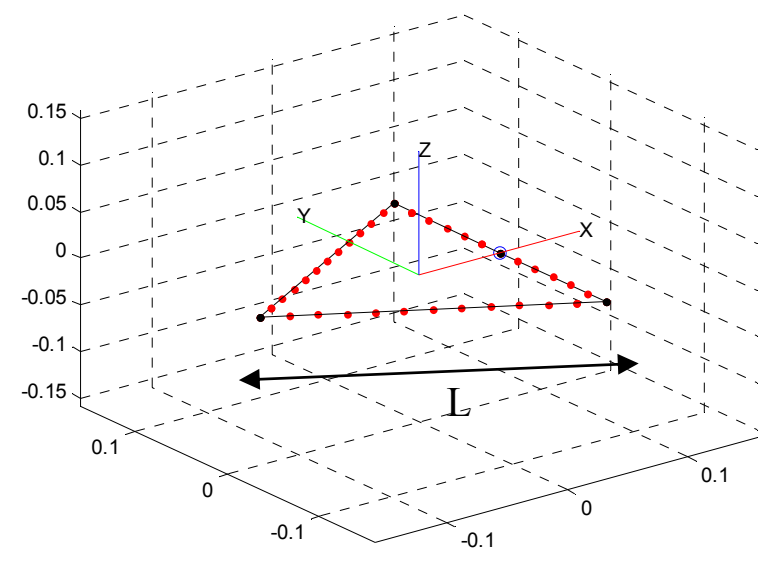

Figure 3.20 Equilateral triangular loop of edge length $L=0.229 \mathrm{~m}$ and wire radius $r=0.5 \mathrm{~mm}$.

Figure 3.21 shows the normalized current distribution at first resonance (480 $\mathrm{MHz}$ ) associated to the first seven characteristic modes of the triangular loop under consideration. Since the shape of the loop is quite different from that of a circular loop, the modal currents also differ significantly from perfect sinusoidal functions, especially currents $J_{3}$ and $J_{4}$. As in the square loop, the current distribution of mode $J_{0}$ is not uniform, with amplitude decreasing at the corners of the loop (segments $6,18,30$ ).

In order to facilitate the interpretation of the current distribution of modes, Figure 3.22 provides approximated schemes of the projection of the current modes along the loop perimeter. Segments are numbered counter clockwise beginning from $X$ axis. At first sight, it seems there is no pair of degenerated modes, since the current distributions of modes with the same number of current nulls do not resemble very much. However, as it is going to be confirmed next, there are two pairs of degenerated modes, modes $J_{1}$ and $J_{2}$, and modes $J_{3}$ and $J_{4}$. 

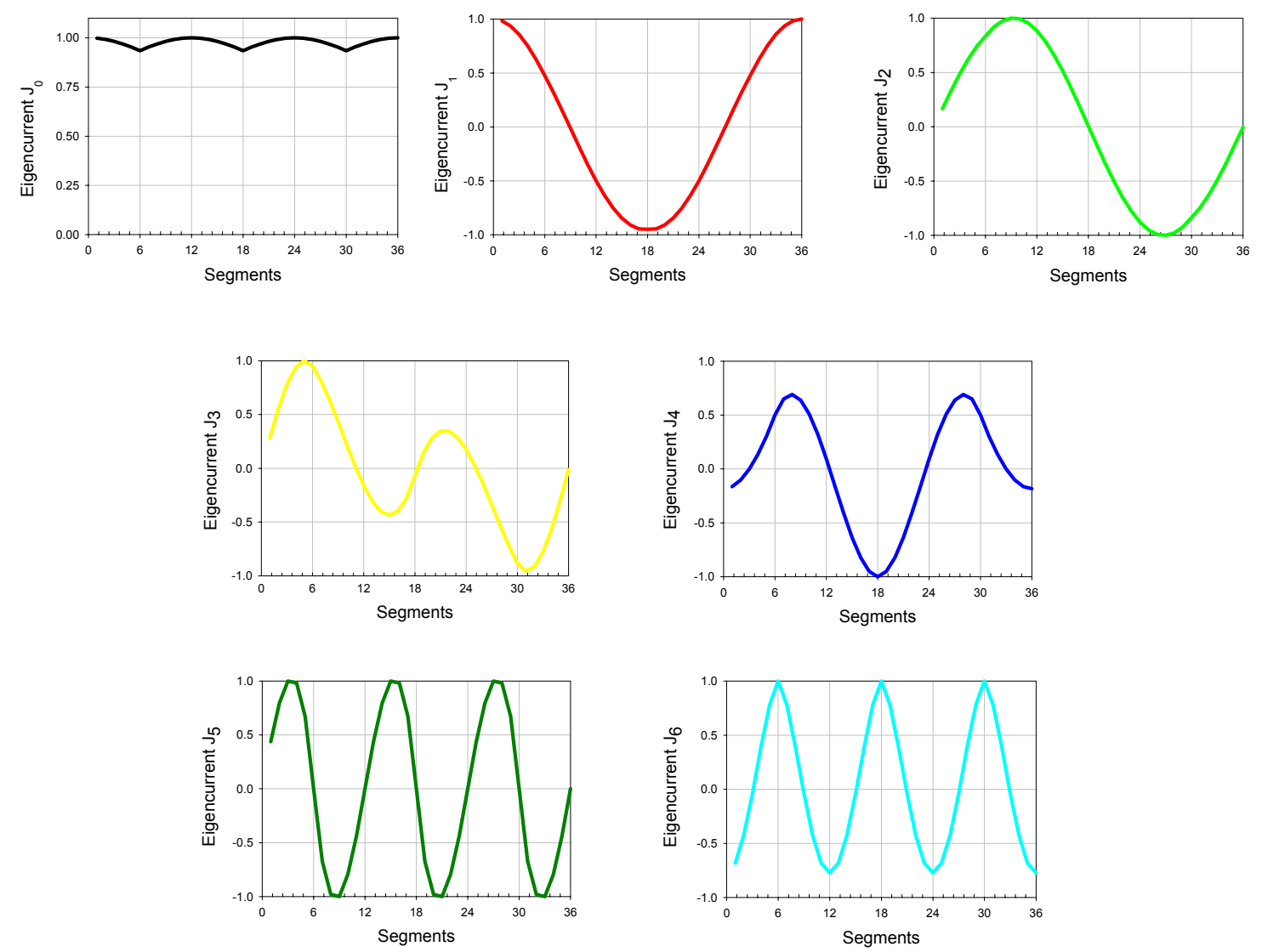

Figure 3.21 Normalized current distribution at first resonance $(f=480 \mathrm{MHz})$ of the first eigencurrents of the triangular loop shown in Figure 3.20.

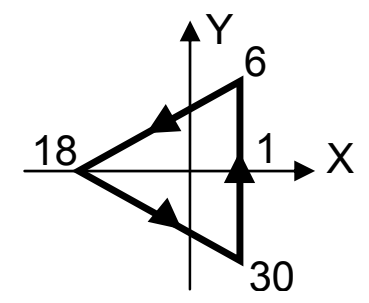

Mode $J_{0}$

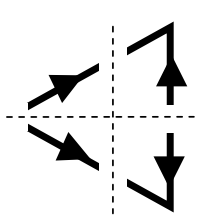

Mode $J_{3}$

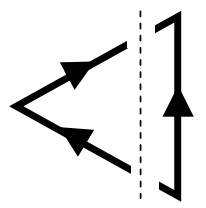

Mode $J_{l}$

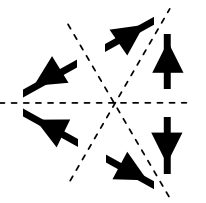

Mode $J_{5}$

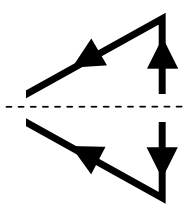

Mode $J_{2}$

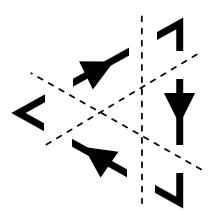

Mode $J_{6}$

Figure 3.22 Schemes of the projection of eigencurrents $J_{n}$ of Figure 3.21 along the perimeter of the triangular loop. 
Figure 3.23 presents characteristic angle variation with frequency for the first seven modes of the triangular loop. From the characteristic angle curves it outcomes that modes $J_{1}$ and $J_{2}$, as well as modes $J_{3}$ and $J_{4}$, present a degeneracy pattern. In contrast, modes $J_{5}$ and $J_{6}$, which are associated to the third harmonic frequency, have split, so each mode presents its own resonance frequency. Table 3.3 includes precise information about the resonance frequency, radiating bandwidth and quality factor of modes.

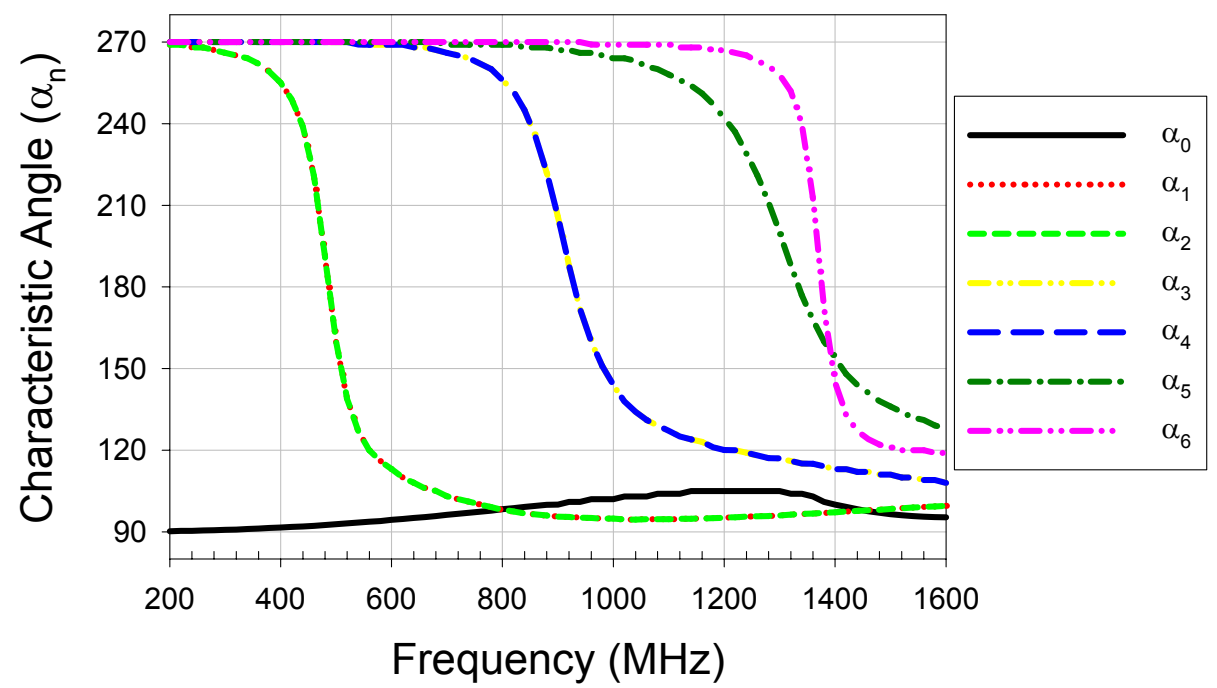

Figure 3.23 Characteristic angle variation with frequency for the first seven modes of the triangular loop shown in Figure 3.20.

\begin{tabular}{|c|c|c|c|c|c|}
\cline { 2 - 6 } \multicolumn{1}{c|}{} & $f_{\text {res }}(\mathrm{MHz})$ & $f_{L}(\mathrm{MHz})$ & $f_{U}(\mathrm{MHz})$ & $B W_{n}(\%)$ & $Q_{\text {rad }, n}$ \\
\hline Modes $J_{1}$ and $J_{2}$ & 490 & 465 & 545 & 16.32 & 6.12 \\
\hline Modes $J_{3}$ and $J_{4}$ & 930 & 885 & 1035 & 16.13 & 6.20 \\
\hline Mode $J_{5}$ & 1335 & 1230 & 1510 & 21 & 4.76 \\
\hline Modes $J_{6}$ & 1375 & 1370 & 1435 & 4.7 & 21.15 \\
\hline
\end{tabular}

Table 3.3. Resonance frequency, radiating bandwidth and quality factor for the first resonant modes of the triangular loop shown in Figure 3.20.

When data for the radiation bandwidth of modes in Table 3.3 is analyzed, it comes out that mode $J_{5}$ is the most efficient radiating mode. This broad radiating bandwidth of mode $J_{5}$, as well as its splitting from mode $J_{6}$, can be once more explained by the corner effect already described in section 3.4.1.

Current schematics in Figure 3.22 show that the current nulls of mode $J_{5}$ hit the three corners of the loop. As a result, the current distribution of mode $J_{5}$ is equivalent to an array of six straight dipoles of length $L / 2$. Conversely, as shown in Figure 3.24, the current distribution of mode $J_{6}$ is equivalent to three straight dipoles of length $L / 2$, and three angle dipoles of equivalent length $L / 4$. 
Evidently, the current distributions of modes $J_{5}$ and $J_{6}$ are not equal, and thus modes are not degenerated. The total effective length of the dipoles that conform the current distribution of mode $J_{6}$, is smaller than that of the dipoles of mode $J_{5}$. This explains why mode $J_{6}$ exhibits higher resonant frequency and smaller radiating bandwidth than mode $J_{5}$.

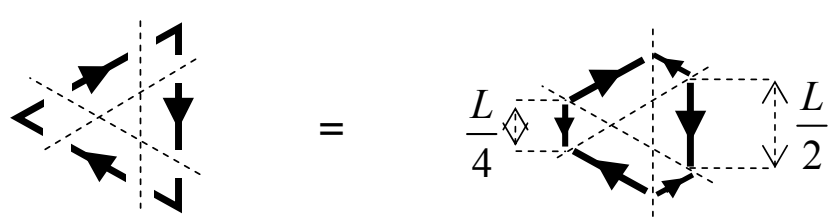

Figure 3.24 Equivalence between the current distribution of mode $J_{6}$ and six dipoles, three of them of length $L / 2$, and the others of length $L / 4$.

Few publications have been found in the available literature concerning the impedance bandwidth of triangular loop antennas [86]-[87]. Nevertheless, from the results presented in [83] it outcomes that, the top-driven regular triangular loop (fed at one vertex) provides the broadest impedance bandwidth when compared with other triangular shapes and other source positions. Let us try to arrive at the same conclusion using characteristic modes.

Figure 3.25 depicts the contribution of the modal VSWR to the total input VSWR of the antenna, for four different positions of a delta gap source. From Figure 3.25 (a) and (c) it is extracted that, the total VSWR obtained for the base-driven loop is always the same, with independence of the arm of the loop that is chosen for placing the source. However, when the source is located at segment 1 only modes $J_{1}, J_{4}$ and $J_{6}$ are excited, whereas when the source is at segment 12, all modes are excited, except mode $J_{5}$. This effect is once again due to the symmetry of the antenna.

The same phenomenon can be observed for the top-driven loop. In Figures 3.25 (b) and (d) it is observed that, the total VSWR obtained when the source is positioned at one vertex of the loop, does not depend on the vertex chosen, although the modes that contribute to this total input $V S W R$ in each case are different.

Note that as stated in [83], the input bandwidth obtained for the top-driven loop is wider than the one given by the base-driven loop. The main difference can be found at the operating band centred at $900 \mathrm{MHz}$, in which the top-driven loop is better matched than the base-driven loop. Nevertheless, mode $J_{5}$ that is the better radiating mode is not excited for neither the base-driven loop nor the top-driven-loop. To excite mode $J_{5}$ the source should be located at one-quarter of the length of the arm, where the mode presents maximum current. 
Figure 3.26 shows the results for the total and modal VSWR when the source is placed at segment 9 . It can be observed that now, due to the excitation of mode $J_{5}$, the third operating band is wider than before, although it is not very well matched to the source. From this result, it is concluded that as affirmed in [83], the top-driven triangular loop provides the wider impedance bandwidth.

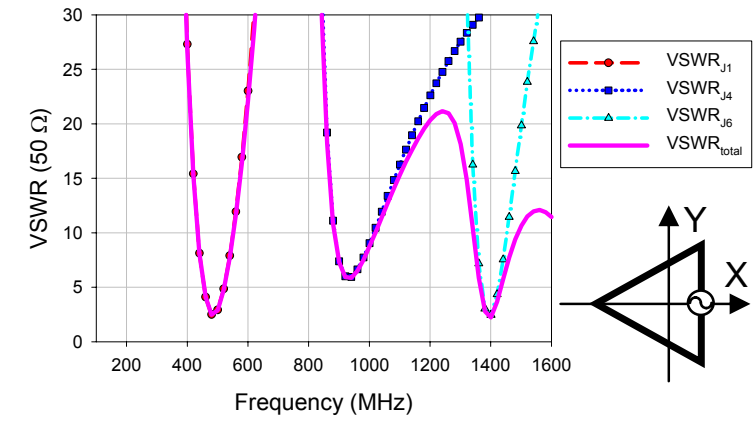

(a) Base-driven loop (source at segment 1)

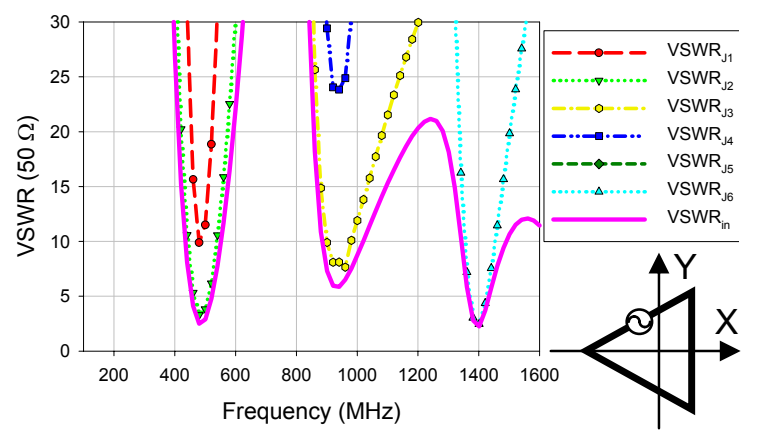

(c) Base-driven loop (source at segment 12)

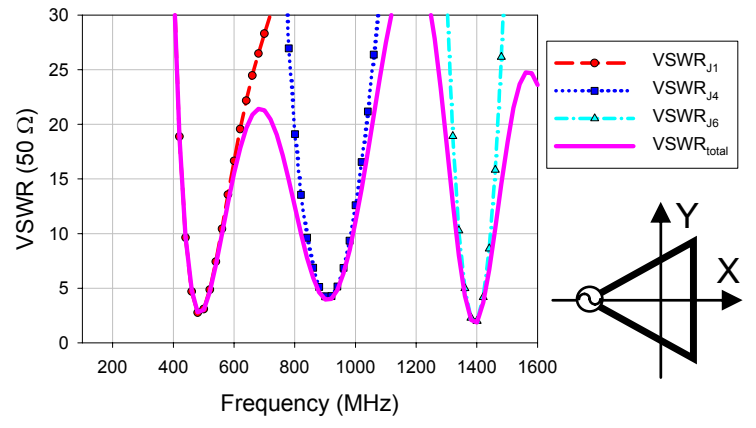

(b) Top-driven loop (source at segment 18)

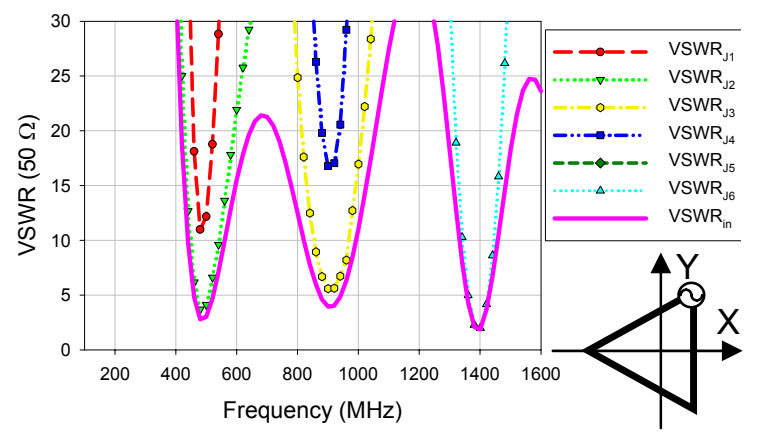

(d) Top-driven loop (source at segment 6)

Figure 3.25 Contribution of the modal VSWR to the total input VSWR for four different positions of a delta gap source.

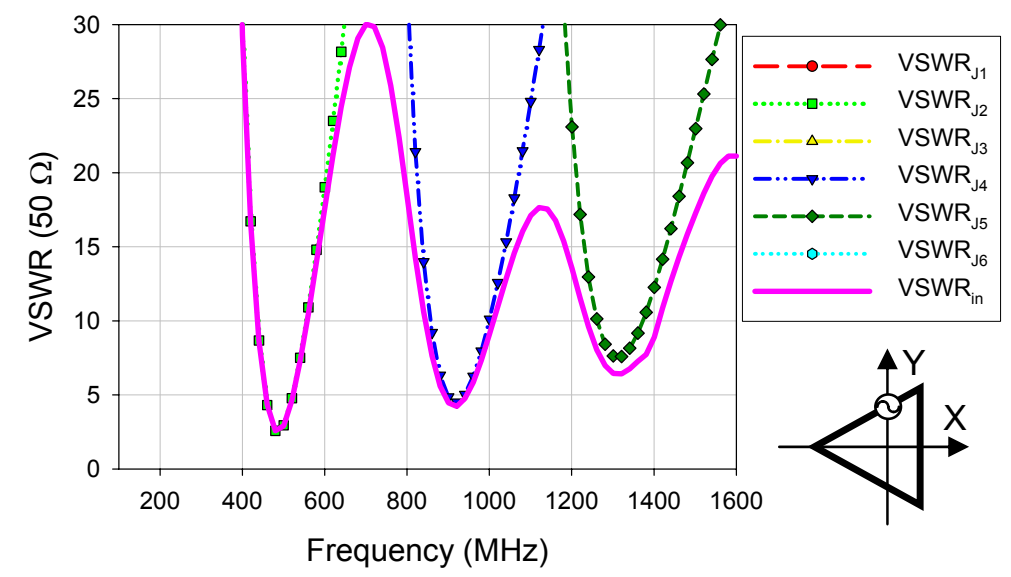

Figure 3.26 Contribution of the modal VSWR to the total input VSWR when a delta gap source is placed at segment 9 of the triangular loop. 


\subsection{Antenna modes and transmission line modes}

In this section, modes are going to be classified in antenna modes or transmission line modes, according to its radiating characteristics. The easiest way to understand this classification of modes is by performing a modal study of an elliptical loop, and drawing a parallel between the modes of this antenna, and the classical antenna and transmission line modes of a folded dipole [86].

Let us begin analyzing what happens to characteristic modes when the geometrical axial ratio $(A R)$ of a circular loop is modified. Figure 3.27 demonstrates how the first two resonant modes of the circular loop studied in section 3.2, deform as the axial ratio of the loop goes from 1 to 0.2 , while the perimeter is preserved. The most remarkable aspect of these current modes is that their deviation from perfect sinusoidal functions increases as the axial ratio decreases. As the axial ratio is reduced, currents associated to mode $J_{l}$ and mode $J_{2}$, resemble more to triangular and a square functions, respectively. In addition, as the axial ratio decreases, degenerated modes of the circular loop split in two modes with radically different radiating behaviour. Next example tackles this concept of mode splitting in more detail.

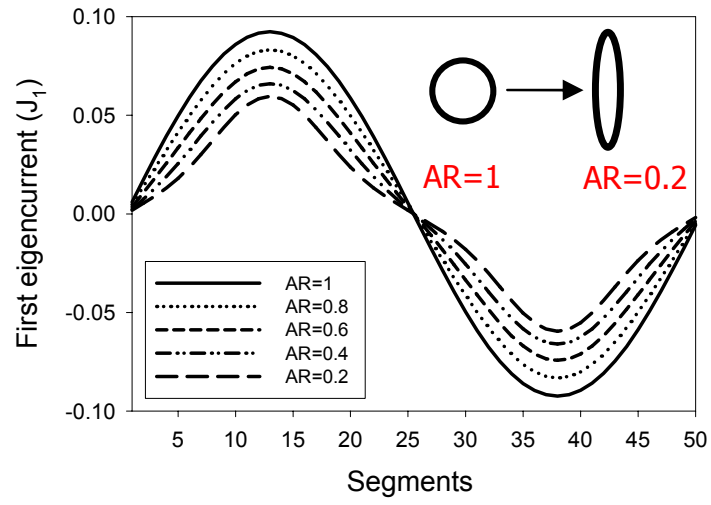

(a) Mode $J_{1}$

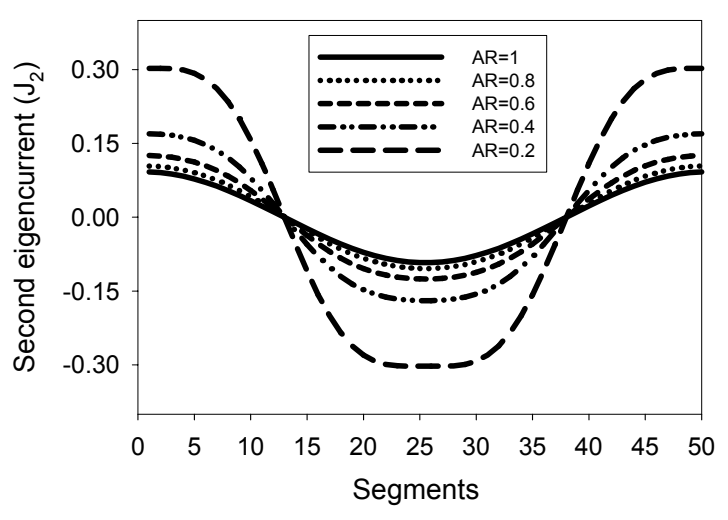

(b) Mode $J_{2}$

Figure 3.27 Evolution of modes $J_{1}$ and $J_{2}$ of the circular loop as its geometrical axial ratio is reduced from 1 to 0.2 .

Consider the elliptical wire loop of axial ratio $A R=0.2$ shown in Figure 3.28. This loop, which is place at $X Y$ plane, presents major axis $A_{\text {major }}=0.3816 \mathrm{~m}$, and minor axis $A_{\text {minor }}=0.0764 \mathrm{~m}$. Because of these axis values the elliptical loop presents the same perimeter as the circular loop reported in section 3.2. Fifty basis functions have been used for expansion and testing in the method of moments calculations. Figure 3.29 illustrates the normalized current distribution of the first five modes of this loop at first resonance $(f=370 \mathrm{MHz})$. As demonstrated in [88], these currents correspond with even and odd angular Mathieu functions. Like in the circular loop, a special inductive mode, $J_{0}$, with nearly uniform current appears. Among the rest of modes, as will be shown next, there are not degenerated pairs. 


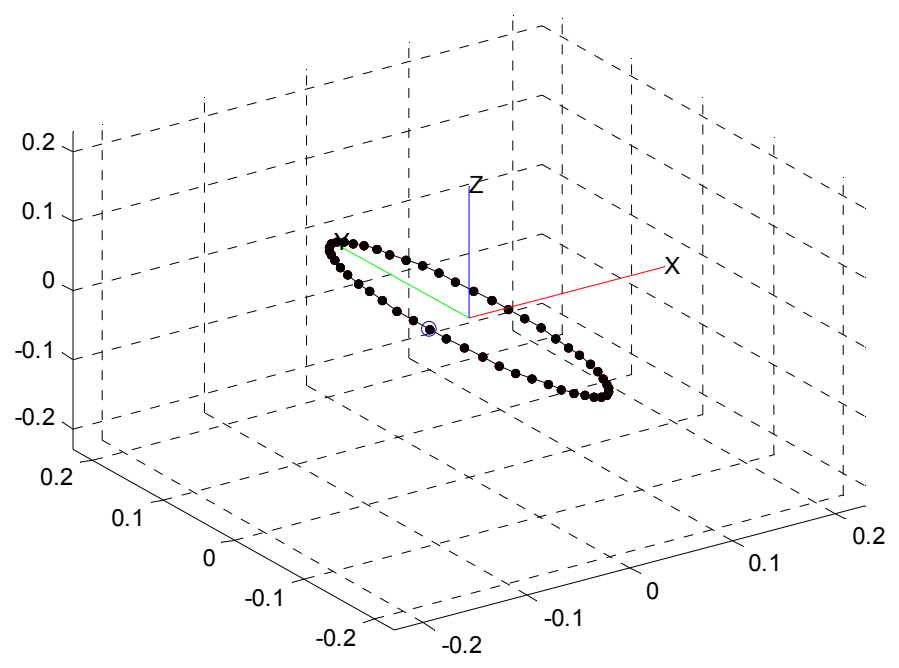

Figure 3.28 Elliptical loop antenna with major axis $A_{\text {major }}=0.3816 \mathrm{~m}$, and minor axis $A_{\text {minor }}=0.0764 \mathrm{~m}$ (axial ratio $A R=0.2$ ).
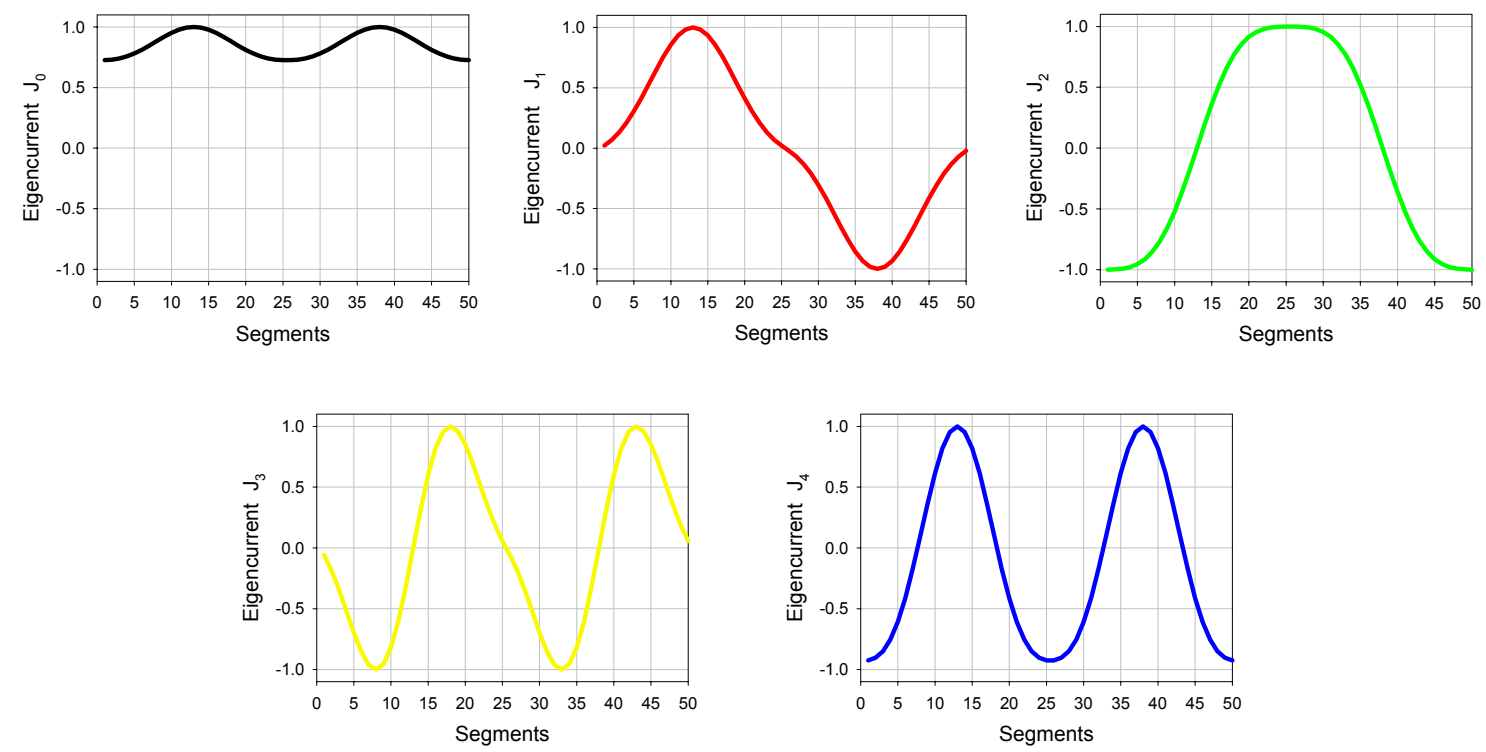

Figure 3.29 Normalized current distribution of the first five modes for an elliptical loop of axial ratio 0.2 at first resonance $(f=370 \mathrm{MHz})$.

Let us concentrate on the behaviour of the first two resonant modes, $J_{I}$ and $J_{2}$, of the elliptical loop. Figure 3.30 supplies schemes of the projection of these eigencurrents over the loop perimeter. Segments are numbered counter clockwise, beginning from $Y$ axis. Figure 3.30 also draws an analogy with the folded dipole, as it compares the first and second eigenvectors of the elliptical loop with the antenna mode and the transmission line mode of the folded dipole. 
Observe that in the antenna mode, current in both arms of the loop flow in phase, so its radiation should reinforce. In contrast, currents in transmission line mode flow with opposite phase, so its radiation should cancel. As a result, the transmission line mode may exhibit poor radiation characteristics and narrow radiating bandwidth, whereas the antenna mode may radiate efficiently. But let us give evidence of this assumption in a more systematic way studying characteristic angle variation with frequency.

Mode $J_{1} \rightarrow$ Antenna Mode

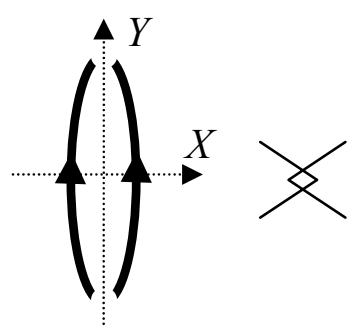

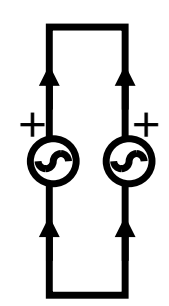

Mode $J_{2} \rightarrow$ Transmission Line Mode
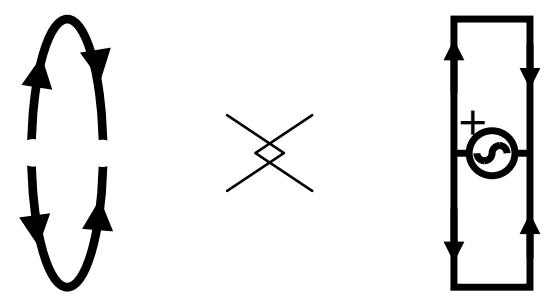

Figure 3.30 Analogy between modes $J_{1}$ and $J_{2}$ of the elliptical loop and transmission line and antenna modes of a folded dipole.

Figure 3.31 analyzes the characteristic angles associated to the first five current modes of the elliptical loop of axial ratio 0.2 . Now, it is evident that there are not degenerated modes, since every mode presents a different resonance frequency. Besides, the characteristic angle behaviour of resonant modes differs quite a lot. Modes with the steepest slope, $J_{2}$ and $J_{4}$, can be identified with transmission line modes that only contribute to radiation at resonance frequency. On the contrary, modes with soft slope near $180^{\circ}, J_{1}$ and $J_{3}$, are antenna modes which contribute to radiation in a wide range of frequencies. This classification of modes according to its characteristic angle confirms the assumption made when analyzing the currents in Figure 3.30.

Just to finish this section, Table 3.4 presents the resonance frequency, bandwidth and quality factor of the first radiating modes of the elliptical loop already analyzed. From these data it is concluded that when degenerated modes of a symmetrical structure split because the geometry of the structure is modified, they transform in two separate modes with different radiating behaviour. According to the slope of their characteristic angle curves, these two previously degenerated modes can be identified with an antenna mode, and a transmission line mode. The more altered the symmetry of the structure is, the more different radiating behaviour these two modes present. 


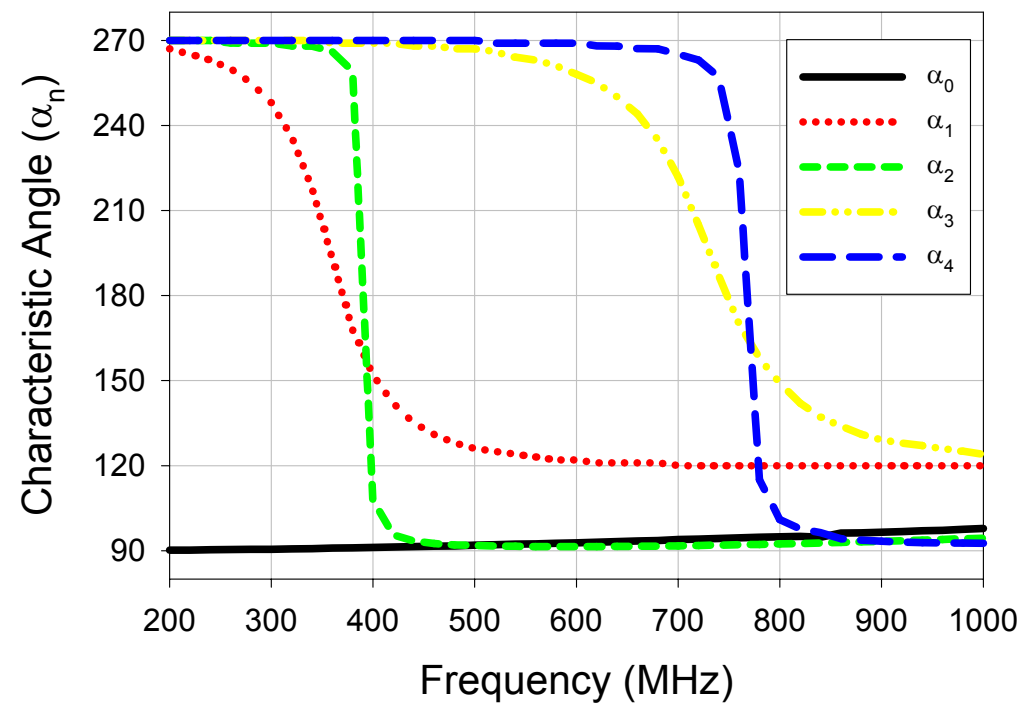

Figure 3.31 Variation with frequency of the characteristic angle $\alpha_{n}$ associated to the first five current modes of the elliptical loop of axial ratio 0.2 .

\begin{tabular}{|c|c|c|c|c|c|}
\cline { 2 - 6 } \multicolumn{1}{c|}{} & $f_{\text {res }}(\mathrm{MHz})$ & $f_{L}(\mathrm{MHz})$ & $f_{U}(\mathrm{MHz})$ & $B W_{n}(\%)$ & $Q_{\text {rad }, n}$ \\
\hline Modes $J_{1}$ & 370 & 330 & 440 & 29.73 & 3.36 \\
\hline Modes $J_{2}$ & 390 & 385 & 395 & 2.56 & 39 \\
\hline Mode $J_{3}$ & 735 & 705 & 855 & 20.41 & 4.90 \\
\hline Modes $J_{4}$ & 770 & 760 & 775 & 1.95 & 51.3 \\
\hline
\end{tabular}

Table 3.4. Resonance frequency, radiating bandwidth and quality factor for the first resonant modes of the elliptical loop shown in Figure 3.28.

Finally, it should be highlighted that a transmission line mode can become an efficient radiator at high frequencies. This is because, far from the resonance of the transmission line mode, currents flowing with opposite phase do not cancel any longer, as the separation between them becomes bigger than $\lambda / 2$. 


\subsection{Modal characterization of complex wire structures.}

Until now, in this work only very simple and well-known wire antennas, like the straight dipole or canonical loops, have been analyzed using characteristic modes. This section is focus on demonstrating that Harrington's Theory can also be very useful for the characterization of arbitrarily shaped wire structures.

Figure 3.32 shows a structure formed by a circular loop of radius $r=0.1145$ $\mathrm{m}$, and a horizontal dipole of length $L=0.229 \mathrm{~m}$. The structure is placed at $X Y$ plane, and it has been discretized in 68 segments, which are numbered counter clockwise beginning from $X$ axis. Segments from 1 to 50 belong to the loop, while segments from 51 to 68 correspond with the dipole. Figure 3.33 depicts the normalized current distribution of the first modes of this complex structure. Note that characteristic currents are quite difficult to analyze now, since there are two junctions in which the loop connects with the dipole. For a better understanding, these currents have been projected along the structure, as shown in Figure 3.34.

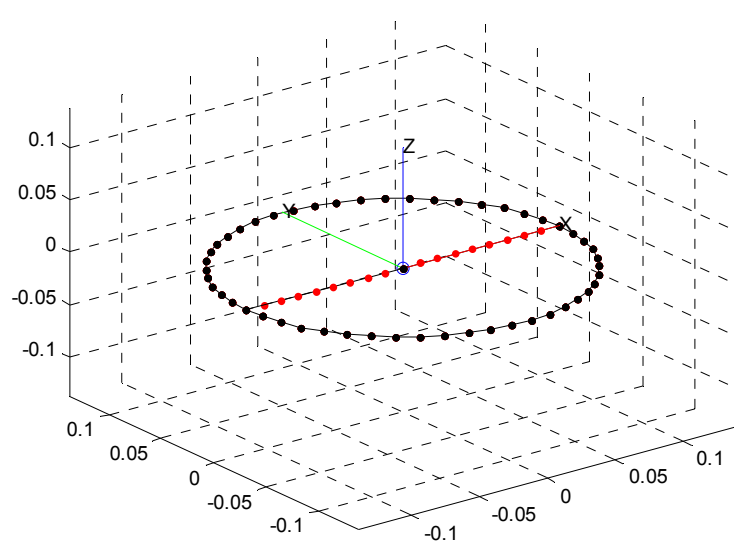

Figure 3.32 Complex antenna formed by a circular loop of radius $r=0.1145 \mathrm{~m}$, and a horizontal dipole of length $L=0.229 \mathrm{~m}$.

Figure 3.35 shows the characteristic angle curves associated to the current modes of Figure 3.33. It should be pointed out that some modes presented in Figure 3.34 are a combination of the already studied modes of the circular loop and the dipole, while other modes just correspond with those obtained for the single circular loop in section 3.2. Observe that there is current flow along the dipole only in modes $J_{2}, J_{4}$, and $J_{5}$. This is because modes of the dipole only couple with those modes of the loop that present zero current at the ends of the dipole. In consequence, in modes $J_{01}, J_{1}$, and $J_{3}$, there is no current flow along the dipole. Note also that as the dipole divides the circular loop in two semicircular loops, in this structure there are two special non-resonant modes, modes $J_{01}$ and $J_{02}$. Mode $J_{01}$ presents currents forming a closed loop around the circular loop. Currents in mode $J_{02}$ form two separate closed loops of semicircular shape. In Figure 3.35 it can be checked that characteristic angles associated to these two special modes are all the time below $180^{\circ}$. 

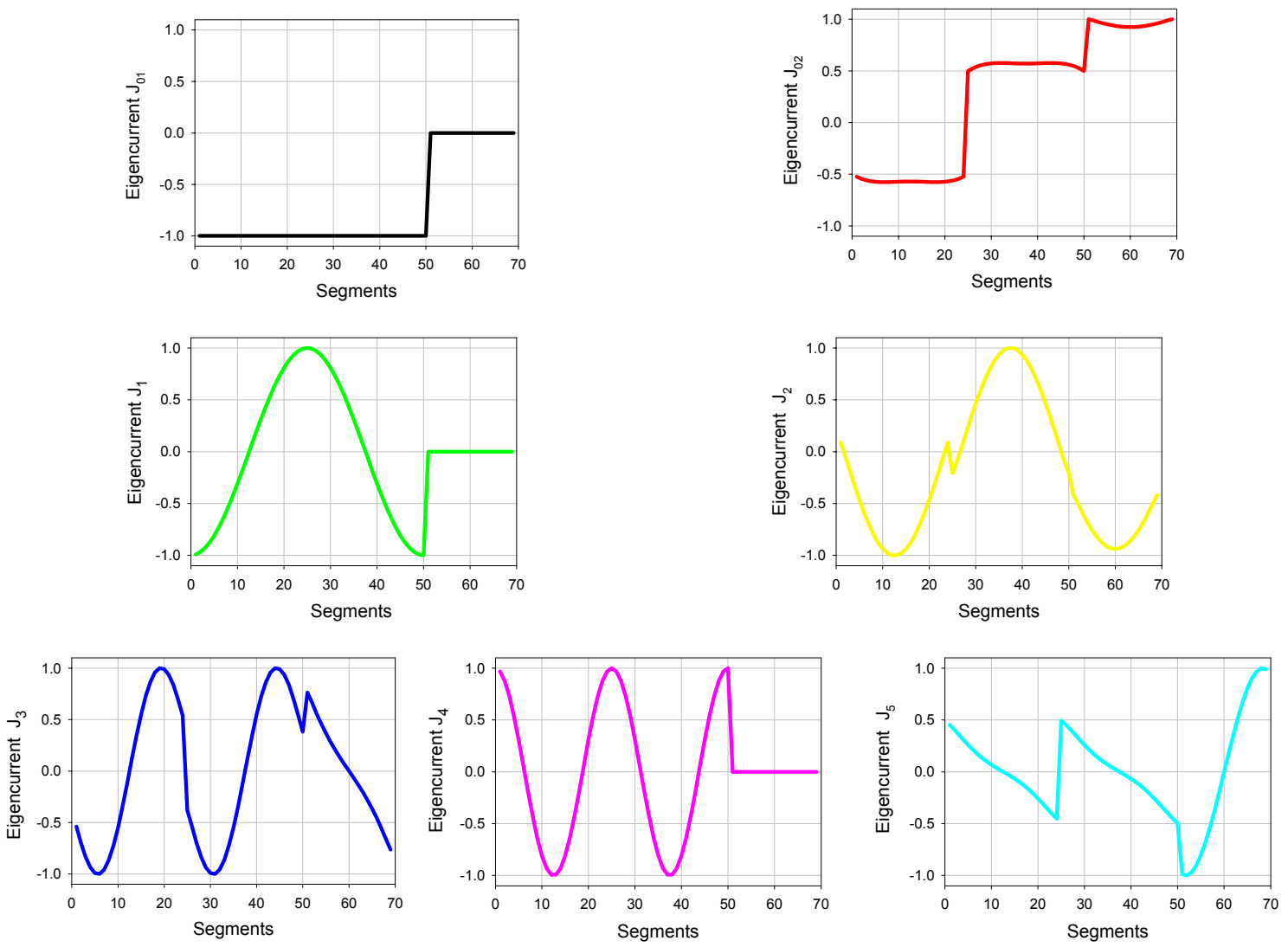

Figure 3.33 Normalized current distribution of the first modes of a structure formed by a circular loop of radius $r=0.1145 \mathrm{~m}$, and a horizontal dipole of length $L=0.229 \mathrm{~m}$.

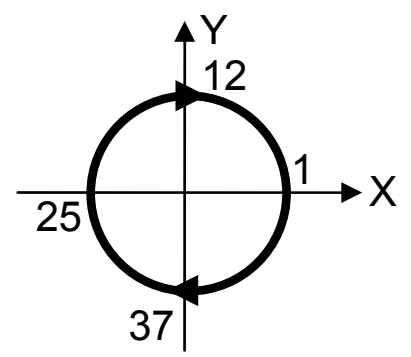

Mode $J_{01}$

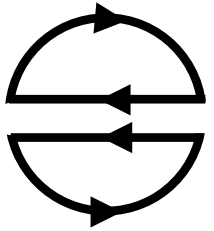

Mode $J_{02}$

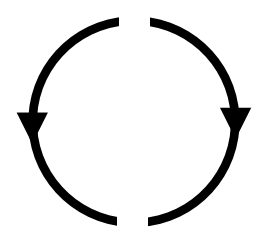

Mode $J_{1}$

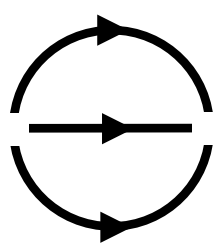

Mode $J_{2}$

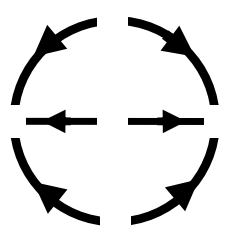

Mode $J_{3}$

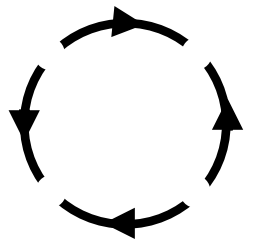

Mode $J_{4}$

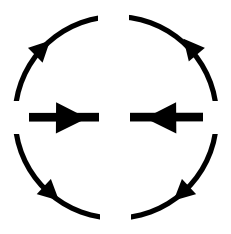

Mode $J_{5}$

Figure 3.34 Schemes of the projection of eigencurrents $J_{n}$ of Figure 3.32 along the structure. 
Information about the resonance frequency, radiating bandwidth, and quality factor of modes, extracted from Figure 3.35, is summarized in Table 3.5. These results evidence that all modes present good radiation bandwidth, except mode $J_{3}$. Mode $J_{3}$ exhibits poor radiation characteristic because it is a transmission line mode, with all currents flowing with opposite phase, and hence, cancelling its radiation. Results obtained for modes $J_{I}$ and $J_{4}$, in which there is no current flow along the dipole, coincide with those obtained in section 3.2 for the first and second pair of degenerated modes of the single circular loop.

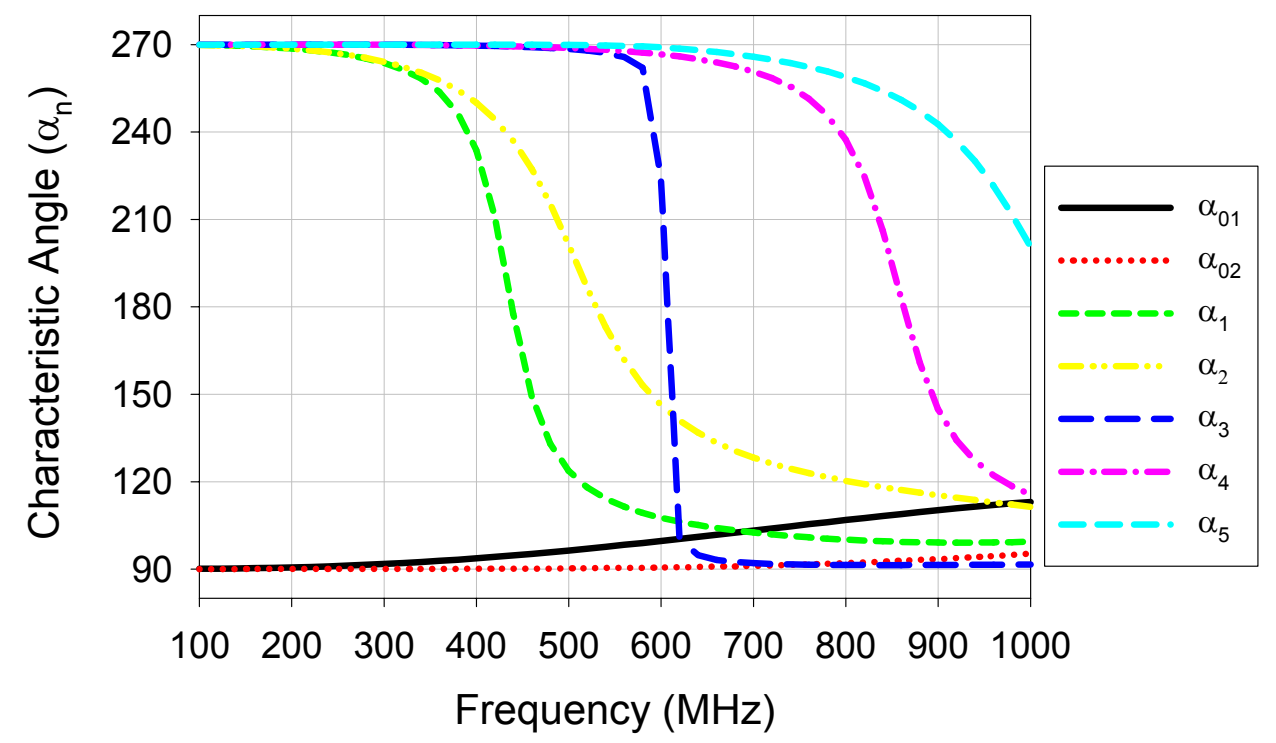

Figure 3.35 Characteristic angle variation with frequency for the current modes shown in Figure 3.33.

\begin{tabular}{|c|c|c|c|c|c|}
\cline { 2 - 6 } \multicolumn{1}{c|}{} & $f_{\text {res }}(\mathrm{MHz})$ & $f_{L}(\mathrm{MHz})$ & $f_{U}(\mathrm{MHz})$ & $B W_{n}(\%)$ & $Q_{\text {rad }, n}$ \\
\hline Modes $\mathrm{J}_{1}$ & 440 & 410 & 475 & 14.77 & 6.77 \\
\hline Modes $\mathrm{J}_{2}$ & 530 & 465 & 655 & 35.85 & 2.79 \\
\hline${\text { Mode } \mathrm{J}_{3}}$ & 605 & 595 & 615 & 3.30 & 30.25 \\
\hline Modes $\mathrm{J}_{4}$ & 860 & 820 & 920 & 11.63 & 8.60 \\
\hline
\end{tabular}

Table 3.5. Resonance frequency, radiating bandwidth and quality factor for the first resonant modes shown in Figure 3.32.

Special attention should be paid to mode $J_{2}$ since it presents very wide radiating bandwidth. This wideband property is due to the fact that mode $J_{2}$ behaves like a three-element folded dipole. A three-element folded dipole acts as an impedance transformer, providing values for the input resistance that are six times those given by a simple dipole working on his fundamental mode. 
An alternative explanation for the wideband behaviour of mode $J_{2}$ is that it results from the combination of two modes with similar radiating behaviour that reinforce each other. Figure 3.36 explains how mode $J_{2}$ can be considered as a sum of the fundamental mode of a dipole (mode $J_{l}$ ), and the fundamental mode of a circular loop (mode $J_{1}$ or $J_{2}$ of the first pair of degenerated modes).

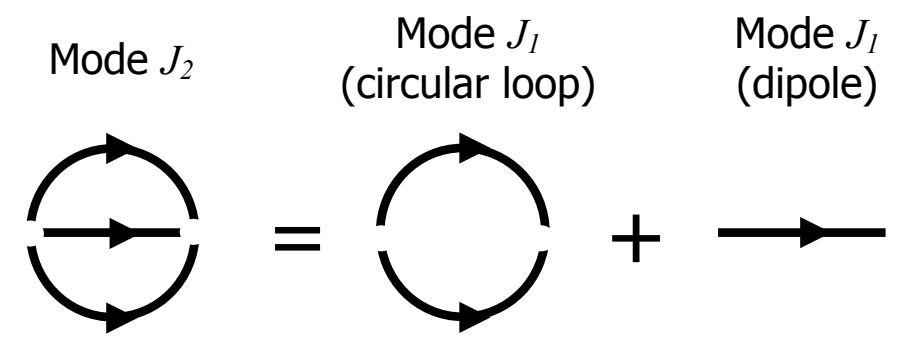

Figure 3.36 Representation of mode $J_{2}$ of the structure under analysis, as a combination of the fundamental modes of the circular loop and the dipole.

Figure 3.37 compares the modal significance curves associated to mode $\mathrm{J}_{1}$ of a dipole of length $L=0.229 \mathrm{~m}$, mode $J_{l}$ of a circular loop of radius $r=0.1145$ $\mathrm{m}$, and mode $J_{2}$ of the complex structure formed by this loop and this dipole. Mode $J_{1}$ of the circular loop resonates at $440 \mathrm{MHz}$, mode $J_{1}$ of the dipole resonates at $620 \mathrm{MHz}$, while mode $J_{2}$ of the complex structure resonates at 530 $\mathrm{MHz}$. The most significant fact is that $530 \mathrm{MHz}$ is the arithmetic mean of 440 and $620 \mathrm{MHz}$. Moreover, the radiating bandwidths of mode $J_{l}$ of the dipole, and mode $J_{1}$ of the circular loop, are $20.16 \%$, and $14.77 \%$, respectively. However, when these two modes combined in a new structure, they yield mode $J_{2}$, which presents an improved radiating bandwidth of $35.85 \%$.

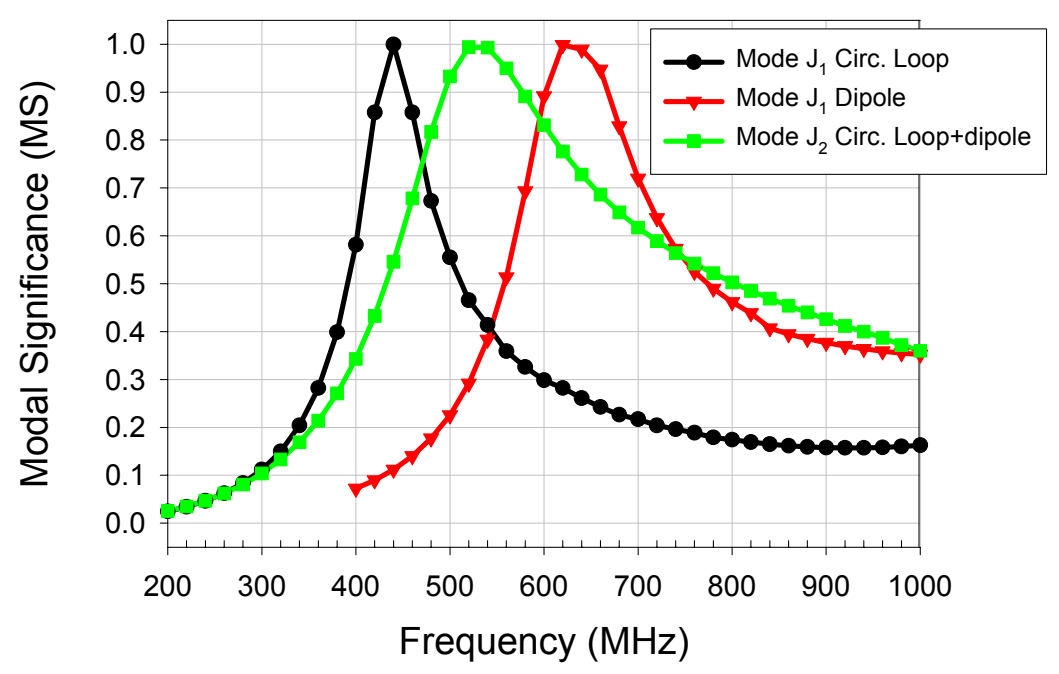

Figure 3.37 Modal significance curves for mode $J_{l}$ of a circular loop, mode $J_{l}$ of a dipole, and mode $J_{2}$ of the structure which results from the combination of this loop and this dipole. 
In order to excite mode $J_{2}$ of the structure under analysis, a delta gap source should be placed at the centre of the dipole. The main advantage of this feeding arrangement is that none of the radiating modes depicted in Figure 3.33, except mode $J_{2}$, will be excited. As a result, mode $J_{2}$ will not interact with any other mode and no antiresonance will appear in the analyzed frequency range.

Figure 3.38 shows the contribution of the VSWR of mode $J_{2}$ to the total input $V S W R$ of the structure, when the source is located at the centre of the dipole. Since mode $J_{2}$ acts as a three-element folded dipole, the VSWRs have been computed for a reference resistance of $426 \Omega$ (six times the input resistance of the simple dipole). As expected, the only radiating mode that is excited is mode $J_{2}$. As a result, an impedance bandwidth of $30.2 \%$ is obtained for $V S W R_{\text {total }}<2$.

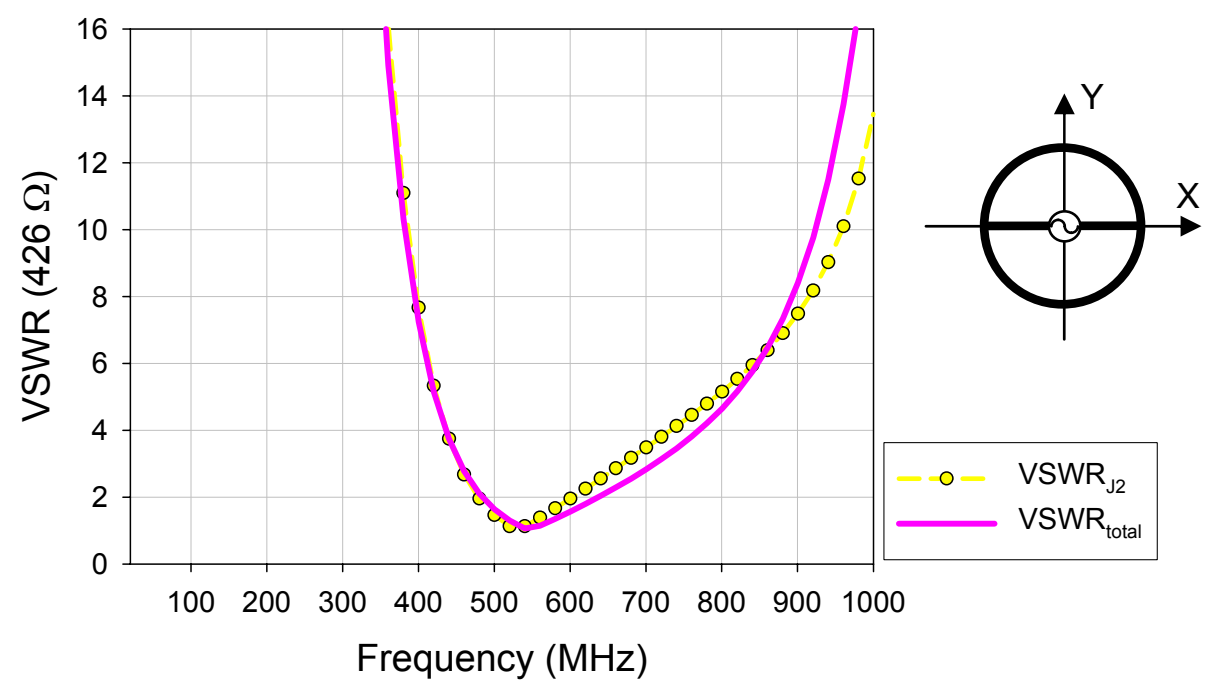

Figure 3.38 Contribution of the modal VSWR of mode $J_{2}$ to the total input $V S W R$ of the antenna when a delta gap source is placed at the centre of the dipole.

The final conclusion of this section is that as a result of combining two simple structures with similar radiating characteristics (same polarization and similar radiation pattern), a new structure with improved input bandwidth can be achieved. Furthermore, these results suggest that wire structures of arbitrary shape can be analyzed as a combination of more simple structures of wellknown behaviour, like dipoles or loops. 
CHAPTER 4. On the Convergence of Characteristic Modes

\subsection{Introduction}

In Chapter 3 characteristic modes were used to analyze several wire antennas with the purpose of determining their basic radiation mechanisms. In that chapter, some results were presented regarding the contribution of modes to the total input admittance of the antenna. However, the accuracy of the results provided by the expansion of modes was not checked.

In contrast with Chapter 3, Chapter 4 will not be focused on the physical insight yield by characteristic modes, but on the problematic derived from the use of Harrington's modes as basis functions in which to expand the total current on the antenna. It will be demonstrated that for antenna problems, the series of characteristic modes presents slow converge when approximating the imaginary part of the input current. As a contribution of this thesis, such slow convergence of the series of modes will be improved by including a new term in the expansion, called "source mode".

On the other hand, the dependency with frequency of characteristic modes will also be addressed in this chapter. Following the approach described in [89], a method based on a Singular Value Decomposition (SVD) [47] will be proposed, in order to obtain a set of frequency independent modes to be used as basis functions in the whole band of interest.

\subsection{The use of characteristic modes as entire-domain basis functions for large-scale electromagnetic problems.}

It is a well-known fact that the electromagnetic analysis of large-size metallic bodies still poses today considerable difficulties, especially when the body presents arbitrary shape, and analytical techniques can not be applied. In this case, numerical approaches are the only remaining alternative.

Among the different numerical approaches, the method of moments (MoM) [42] is the most widespread formulation for antenna problems. It is a common practice to use Rao, Wilton and Glisson (RWG) basis functions [44] in the conventional MoM analysis. These subdomain basis functions are very flexible, as they can be applied to any kind of geometry. However, at least ten of these basis functions per wavelength are required to obtain accurate solutions. Since the dimension of the moment matrix is equal to the number of employed basis functions, as the electric size of the object increases, the number of unknowns grows very rapidly. As a result, it is necessary to deal with huge matrix equations that need long computational time and large memory requirements to be solved. 
Some of the attempts that have been made in order to reduce the size of the matrix equations arising in the MoM analysis of large scale problems, consist of using high-level basis functions, like the Macro Basis Functions (MBFs) proposed in [48], or the Characteristic Basis Functions (CBFs) described in [90]. The basic idea of both these methods is to divide the complex geometry into simpler parts, and then to define a set of sub-sectional basis functions for each subdomain. Finally, the partial solutions obtained by direct inversion, are combined to form a compressed global moment system.

A more efficient alternative to overcome the problem is to use entire-domain basis functions [91]-[92] in the MoM analysis. These basis functions extend all over the surface of the body. The main problem of these functions is that they are really dependent on the geometry of the body. The optimal choice of the set of functions is one that resembles the unknown function and leads to convergent solutions with the fewest number of terms in the expansion, and subject to the shortest computational time. Examples of entire-domain basis functions are Fourier trigonometric functions, and Legendre, Hermite and Chebyshev polynomials [93].

On the basis of the previous discussion, it could be considered that when Harrington and Mautz defined characteristic modes in the seventies, they found the perfect candidates to be used as entire-domain basis functions to expand the unknown current on the surface of arbitrarily shaped conducting bodies. Since characteristic modes correspond with the natural resonances of the body, they incorporate the physics of the problem, and they fit its geometry in perfect way. Moreover, characteristic modes can be obtained numerically for any antenna shape, so there are not restricted to canonical structures, and due to their orthogonality properties, already described in Chapter 2, they provide wellconditioned MoM matrices.

Hence, characteristic modes seem particularly suitable to reduce the computational cost when analyzing large electromagnetic problems, especially for the case of large arrays, in which the number of elements, other than their electric size, becomes an important factor affecting the complexity of the solution. For example, let us suppose an array formed by $P$ identical microstrip patches. If this array were analyzed by the conventional MoM using $n_{s}$ subdomain basis functions in each patch, it would lead to a problem with $N_{s}=n_{s} \cdot P$ unknowns. The computational cost for such a problem would be of the order of $O\left(N_{s}^{3}\right)$. Obviously, the number of unknowns becomes prohibitive if the number or the size of the elements increases too much. Conversely, if characteristic modes were used as entire-domain basis functions, the number of unknowns would reduce to $N_{c}=n_{c} \cdot P$, where $n_{c}$ is the number of modes used for the current expansion. The computational cost would be now $O\left(N_{c}^{3}\right)+O\left(N_{s} \cdot n_{c}^{2}\right)$, where the additional term would account for the computation of the characteristic modes of a single patch. Having into account that $n_{c}<<n_{s}$, the advantage of the second method is evident. 
But, why characteristic modes have not been generally accepted as the optimum entire-domain basis functions for large scale problems? The answer to this question is that characteristic modes present several drawbacks when they are used as basis functions to expand the total current. First one is that although characteristic modes have been demonstrated to be very effective in the analysis of scattering from conducting objects [94]-[95], they present very slow convergence when they are applied to antenna problems. The reason for this slow convergence lies in the very definition of characteristic modes. Characteristic modes are computed in the absence of any source or excitation, so they are only constrained by boundary conditions, and they do not take into account the coupling between the excitation and the structure. Then, characteristic modes can not account properly for near-field effects, and they are not appropriate for the modelling of the particular behaviour of the current at the feed point. As it was demonstrated in [96], when a delta gap source is placed over an antenna, characteristic modes sometimes lead to even non convergent solutions for the imaginary component of the total current, and consequently for the input impedance.

The second drawback of characteristic modes, probably less reported in the literature than the previous one, is their dependency upon frequency, which prevents them from being used as wideband basis functions.

In the following sections these negative aspects of characteristic modes are going to be studied thoroughly, and solutions for them are going to be proposed.

\subsection{Slow convergence of the series of characteristic modes for wire antennas.}

As it was mentioned above, characteristic modes associated to a particular radiating structure do not model properly the imaginary or non-radiating component of the current at the feed point of the antenna. To illustrate this problem, the wire dipole of length $L=0.5 \mathrm{~m}$ and wire radius $r=0.5 \mathrm{~mm}$, already studied in Chapter 2, is going to be studied again.

Figure 4.1 compares the actual total current of the centre fed dipole at first resonance $(280 \mathrm{MHz}$ ) with the one obtained using the modal expansion described in equation (2.21) of Chapter 2. Modes with odd current distribution have not been considered, because for the centre-fed dipole only even modes are excited. As can be observed in Figure 4.1, a very good approximation for the real part of the current is obtained just using the first characteristic mode of the dipole. On the contrary, the modal approximation fails to model the imaginary part of the current, especially at the feeding point (segment 50), even when five even characteristic modes are used for the expansion. Note that it is really important to obtain an accurate approximation for the current at feeding point, since the input current is commonly used to determine some basic antenna parameters, such as the input impedance and the return loss. 


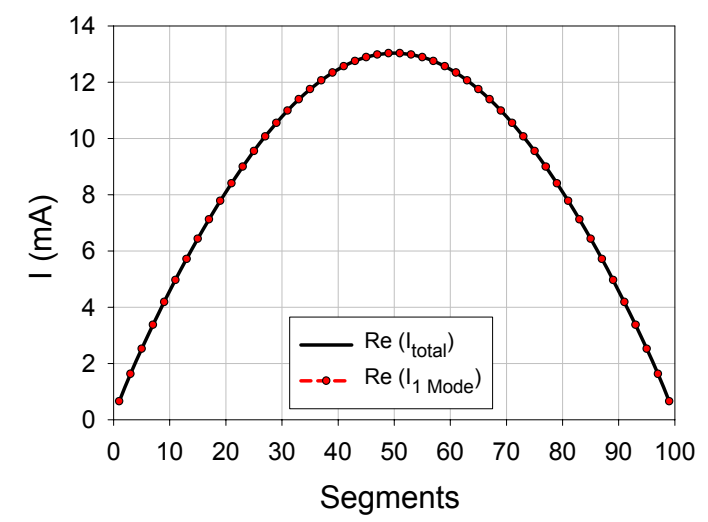

(a) Real part of the current.

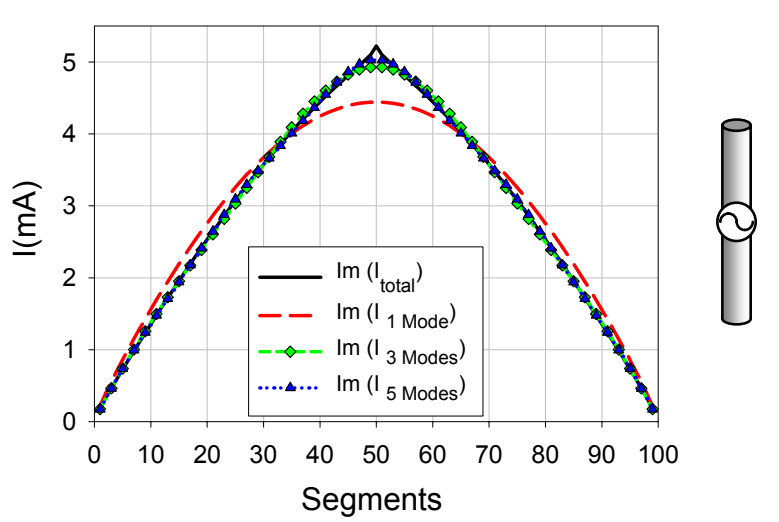

(b) Imaginary part

Figure 4.1 Comparison of the actual total current of a centre-fed dipole and the current obtained with a modal expansion of one, three and five even modes.

Table 4.1 shows the relative quadratic error computed at $280 \mathrm{MHz}$ when the total input current of the centre fed dipole is approximated by an expansion with variable number of even modes. It can be observed that to obtain a relative error less than $1 \%$ for the imaginary part of the input current, it is necessary to consider more than 20 even modes in the expansion.

A possible explanation for the results presented in Figure 4.1 and Table 4.1, is the different nature of the real and imaginary parts of the current. On one hand, the real part of the current, which is the one responsible for radiation, only depends on the resonances of the structure, and do not depend on the feeding arrangement. Just the opposite, the imaginary part or non-radiating part of the current, is very dependent on the position of the delta gap source. Hence, because of its radiating nature, the real part of the current can be easily approximated at first resonance, just using the first radiating mode of the dipole. In contrast, characteristic modes are unable to model precisely the current peak exhibited by the imaginary current at the feed point.

\begin{tabular}{|c|c|c|}
\hline $\begin{array}{c}\text { Number of even } \\
\text { modes }\end{array}$ & $\begin{array}{c}\text { \% Relative quadratic error } \\
\text { Real part }\end{array}$ & $\begin{array}{c}\text { \% Relative quadratic error } \\
\text { Imaginary part }\end{array}$ \\
\hline 1 & $1.48 \mathrm{e}-4$ & 14.94 \\
\hline 2 & $2.03 \mathrm{e}-7$ & 9.36 \\
\hline 3 & $1.42 \mathrm{e}-7$ & 5.56 \\
\hline 5 & $1.07 \mathrm{e}-7$ & 3.63 \\
\hline 20 & $4.72 \mathrm{e}-8$ & 1.16 \\
\hline 30 & $2.94 \mathrm{e}-8$ & 0.67 \\
\hline
\end{tabular}

Table 4.1. Relative quadratic error computed at $280 \mathrm{MHz}$ when the total input current of the centre fed dipole is approximated by an expansion with variable number of even modes. 
With the aim of verifying the dependency of the imaginary part of the current with the position of the source, Figure 4.2 shows the modal approximation at $280 \mathrm{MHz}$ for the current of the same dipole of length $L=0.5 \mathrm{~m}$, when it is fed by a delta gap source placed at segment 25 . In this case, both even and odd modes have been considered for the expansion of the current. As observed, the real part is not affected by the position of the source, since it is equal to the one obtained in Figure 4.1 for the centre fed dipole. Once again, this real part can be approximated in a precise way using only the first radiating mode of the dipole. In contrast, the imaginary part of the current presents a peak at the location of the source. As the number of modes used to approximate this imaginary part increases, the current resembles more the total current, yet it still fails to converge at the feed point.

Table 4.2 presents the relative quadratic error calculated at $280 \mathrm{MHz}$ for the total input current of the dipole fed at segment 25 , when it is approximated with an expansion of increasing number of modes. From these results it comes up that to obtain an error less than $1 \%$, more than 40 modes are needed.

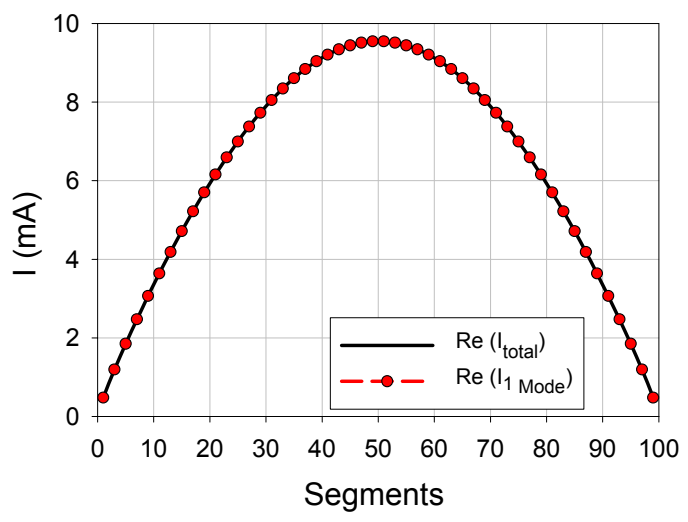

(a) Real part of the current.

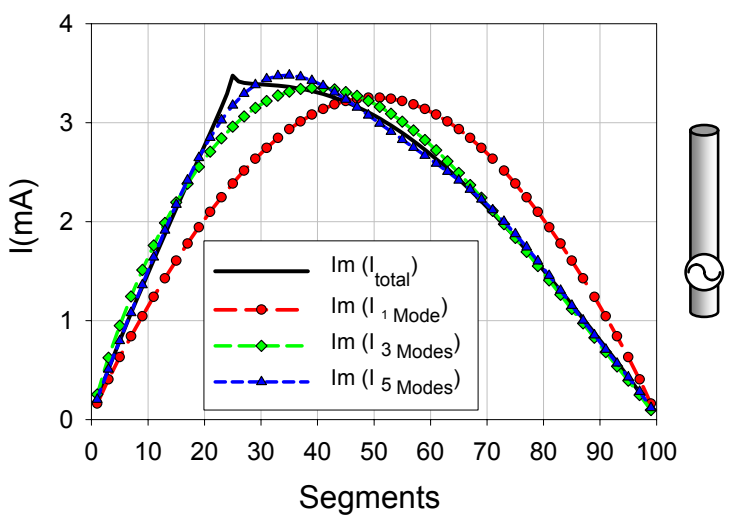

(b) Imaginary part

Figure 4.2 Comparison of the actual total current of a centre-fed dipole and the current obtained for a modal expansion of one, three and five modes.

\begin{tabular}{|c|c|c|}
\hline $\begin{array}{c}\text { Number of } \\
\text { modes }\end{array}$ & $\begin{array}{c}\text { \% Relative quadratic error } \\
\text { Real part }\end{array}$ & $\begin{array}{c}\text { \% Relative quadratic error } \\
\text { Imaginary part }\end{array}$ \\
\hline 1 & $38.56 \mathrm{e}-3$ & 31.38 \\
\hline 2 & $1.30 \mathrm{e}-5$ & 15.20 \\
\hline 3 & $3.10 \mathrm{e}-7$ & 14.82 \\
\hline 5 & $2.7 \mathrm{e}-7$ & 8.65 \\
\hline 20 & $1.38 \mathrm{e}-7$ & 3.22 \\
\hline 40 & $8.65 \mathrm{e}-8$ & 1.72 \\
\hline
\end{tabular}

Table 4.2. Relative quadratic error computed at $280 \mathrm{MHz}$ when the total input current of a dipole fed at segment 25 is approximated with an expansion of increasing number of modes. 


\subsection{The source mode for wire antennas.}

In previous section it has been demonstrated that when a wire antenna is fed by a delta gap source, the imaginary part of its current presents a current peak at the feed point, in which characteristic modes fail to converge in a fast way. This section is aimed at determining more precisely, which is the effect of the delta gap source over the total current, in order to find out a solution for reducing the number of modes needed to obtain an accurate approximation for the current at the feed point.

Figure 4.3 shows the residue that is left after subtracting a series of five even characteristic modes from the actual total current at $280 \mathrm{MHz}$ of the centre-fed dipole analyzed in section 4.3 . Observe that this residue resembles a travelling wave with a high peak at the feed point (segment 50), and with a rapidly vanishing profile away from it. Since characteristic modes are mainly associated to radiating power, the primary content of this residue must be reactive. As can be seen in Figure 4.3, this function is nearly singular at the feed point. This type of behaviour is due solely to the presence of a source in the structure, and it limits severely the convergence of the series of characteristic modes. Due to the travelling wave nature of this evanescent mode, it is not an easy task to single it out analytically from the eigenvalue problem used to compute characteristic modes. Antennas are resonant structures, and characteristic modes, which are its resonances, are standing waves by definition.

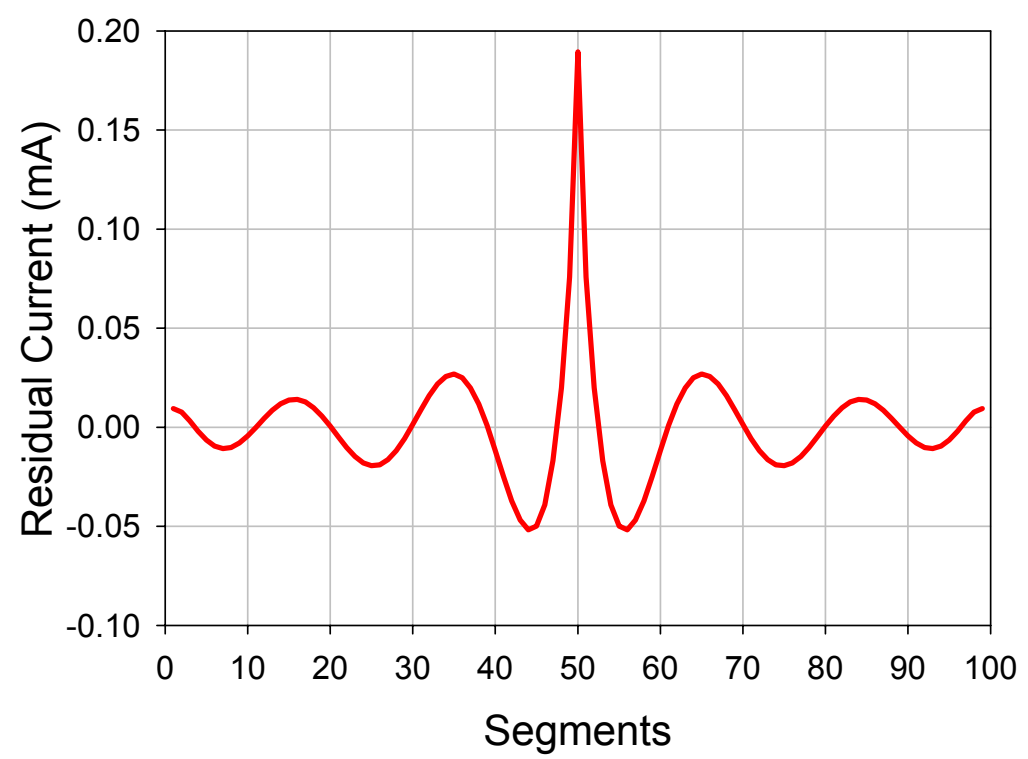

Figure 4.3 Residual current at $280 \mathrm{MHz}$ obtained after subtracting five even characteristic modes from the total current of a centre fed dipole. 
In order to investigate in more detail the nature of this mode, let us consider an infinite wire of radius $a$, fed by a magnetic frill source of radius $b$, as shown in Figure 4.4. All possible current modes along this structure are travelling waves. Therefore, there should be an evanescent travelling-wave mode in the vicinity of the source like in finite wires. The analysis of this evanescent mode will help to find a solution for the convergence problem of the series of characteristic modes.

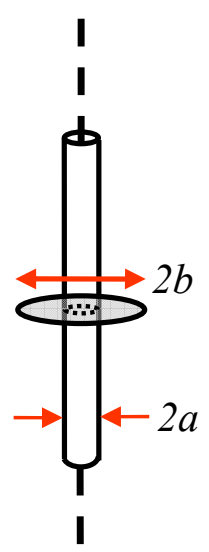

Figure 4.4 Infinite conducting wire of radius $a$, fed by a magnetic frill of radius $b$.

The evanescent current created by the magnetic frill over the cylindrical wire can be derived from the total magnetic field over the wire surface. This magnetic field due to the frill can be obtained as a superposition of magnetic rings of radius $\rho$ ' between $a$ and $b$.

The magnetic field radiated by a ring source of radius $\rho$ ' is known in spectral form [97]

$$
H_{\phi}^{i}=-\frac{\omega \varepsilon P_{0}}{4} \int_{-\infty}^{\infty} H_{1}^{(2)}\left(k_{\rho} \rho^{\prime}\right) J_{1}\left(k_{\rho} \rho\right) e^{j k_{z} z} d k_{z} \quad \rho<\rho^{\prime}
$$

where $k_{\rho}=\sqrt{k^{2}-k_{z}^{2}}$ and $P_{0}$ is a magnetic current moment in volt. meters.

Then, the incident electric field can be deduced from (4.1) as follows

$$
E_{z}^{i}=\frac{1}{j \omega \varepsilon \rho} \cdot \frac{\partial}{\partial \rho}\left(\rho H_{\phi}^{i}\right)=j \frac{P_{0}}{4} \int_{-\infty}^{\infty} k_{\rho} H_{1}^{(2)}\left(k_{\rho} \rho^{\prime}\right) J_{0}\left(k_{\rho} \rho\right) e^{j k_{z} z} d k_{z}
$$

According to the definition of the Fourier Transform (FT) the incident electric field spectrum is given by

$$
\tilde{E}_{z}^{i}=j \frac{\pi P_{0}}{2} k_{\rho} H_{1}^{(2)}\left(k_{\rho} \rho^{\prime}\right) J_{0}\left(k_{\rho} \rho\right)
$$


Enforcing zero tangential electric field at the wire surface $(\rho=a)$, the spectrum of the scattered electric field can be expressed as

$$
\tilde{E}_{z}^{s}=\frac{-j \pi P_{0}}{2} k_{\rho} H_{1}^{(2)}\left(k_{\rho} \rho^{\prime}\right) \frac{J_{0}\left(k_{\rho} a\right)}{H_{0}^{(2)}\left(k_{\rho} a\right)} H_{0}^{(2)}\left(k_{\rho} \rho\right)
$$

Therefore, the total electric field spectrum takes the form of

$$
\tilde{E}_{z}^{t}=\tilde{E}_{z}^{i}+\tilde{E}_{z}^{s}=\frac{j \pi P_{0}}{2} k_{\rho} H_{1}^{(2)}\left(k_{\rho} \rho^{\prime}\right)\left(J_{0}\left(k_{\rho} \rho\right)-\frac{J_{0}\left(k_{\rho} a\right)}{H_{0}^{(2)}\left(k_{\rho} a\right)} H_{0}^{(2)}\left(k_{\rho} \rho\right)\right)
$$

The total magnetic field spectrum at the wire surface is then obtained from (4.5) as

$$
\left.\tilde{H}_{\phi}^{t}\right|_{\rho=a}=\left.\frac{1}{j \omega \mu} \frac{\partial \tilde{E}_{z}^{t}}{\partial \rho}\right|_{\rho=a}=\frac{j P_{0}}{k \eta a} k_{\rho} \frac{H_{1}^{(2)}\left(k_{\rho} \rho^{\prime}\right)}{H_{0}^{(2)}\left(k_{\rho} a\right)}
$$

The total magnetic field radiated by the frill of radius $b$, can be obtained integrating the magnetic field caused by different magnetic rings of radius $a<\rho^{\prime}<b$

$$
H_{\phi}^{t}=\frac{j V_{0}}{k \eta \ln \left(\frac{b}{a}\right)} \cdot \frac{1}{2 \pi a} \int_{-\infty}^{+\infty} k_{\rho} \frac{1}{H_{0}^{(2)}\left(k_{\rho} a\right)} \cdot\left[\int_{\rho^{\prime}=a}^{\rho^{\prime}=b} H_{1}^{(2)}\left(k_{\rho} \rho^{\prime}\right) d \rho^{\prime}\right] e^{j k_{z} z} d k_{z}
$$

After some manipulations, the total magnetic field created by the frill at the wire surface can be expressed as

$$
H_{\phi}^{t}=\frac{j 2 V_{0}}{k \eta \ln \left(\frac{b}{a}\right)} \cdot \frac{1}{2 \pi a}\left[\int_{0}^{k}\left(1-\frac{H_{0}^{(2)}\left(k_{\rho} b\right)}{H_{0}^{(2)}\left(k_{\rho} a\right)}\right) e^{j k_{z} z} d k_{z}+\int_{k}^{\infty}\left(1-\frac{K_{0}(\alpha b)}{K_{0}(\alpha a)}\right) e^{j k_{z} z} d k_{z}\right]
$$

where $\alpha=\sqrt{k_{z}^{2}-k^{2}}$.

The current flowing along the infinite wire will be proportional to the total magnetic field in (4.8)

$$
I(z)=2 \pi a \cdot J(z)=\frac{j 2 V_{0}}{k \eta \ln \left(\frac{b}{a}\right)}\left[\int_{0}^{k}\left(1-\frac{H_{0}^{(2)}\left(k_{\rho} b\right)}{H_{0}^{(2)}\left(k_{\rho} a\right)}\right) e^{j k_{z} z} d k_{z}+\int_{k}^{\infty}\left(1-\frac{K_{0}(\alpha b)}{K_{0}(\alpha a)}\right) e^{j k_{z} z} d k_{z}\right]
$$

with $J(z)=\hat{n} \times H_{\phi}^{t} \hat{\phi}$ 
Two contributions can be identified in the spectral representation of the current presented in (4.9). The first term is the main contributor to the total current, and if the wire were finite it would be responsible for the resonant modes present at the dipole. On the other hand, the second term represents an evanescent current, which is pure imaginary. This second term can be easily obtained in closed form using just a few exponentials by the Generalized Pencilof-Function method (GPOF) [98]. The resulting evanescent current is then

$$
I^{\text {evan }}(z)=\frac{j 2 V_{0}}{k \eta \ln \left(\frac{b}{a}\right)} \operatorname{Re}\left[\int_{k}^{+\infty}\left(1-\frac{K_{0}(\alpha b)}{K_{0}(\alpha a)}\right) e^{j k_{z} z} d k_{z}\right]=\frac{j 2 V_{0}}{k \eta \ln \left(\frac{b}{a}\right)} \sum_{i=1}^{M} \frac{\alpha_{i}}{\beta_{i}-j z} e^{-\left(\beta_{i}-j z\right) k}
$$

Figure 4.5 shows the evanescent current obtained from equation (4.10) for an infinite wire of radius $a=0.5 \mathrm{~mm}$, fed at its centre by a frill of radius $b=5$ $\mathrm{mm}$. This reactive current exhibit a peak at the frill location, and due to its vanishing oscillatory behaviour, it resembles quite a lot the residual current obtained numerically in Figure 4.3. In fact, current in Figure 4.5 can be considered as an evanescent current mode linked to the source. It is expected that the addition of this mode, called from now on "source mode", to the series of characteristic modes will improve its convergence significantly.

Nevertheless, the analytical calculation of the source mode for different wire antennas and different positions of the source is very complex. Alternatively, the source mode can be approximated by an exponential function, capable of accounting for the effect of the source over the current of the antenna. Figure 4.6 shows the exponential source modes proposed for the dipoles already analyzed in section 4.3 . For a conducting wire of radius $r=0.5 \mathrm{~mm}$, the optimum choice for the source mode has been found to be an exponential of argument $2 \pi n$ over the number of segments that separate the source from the closest open end.

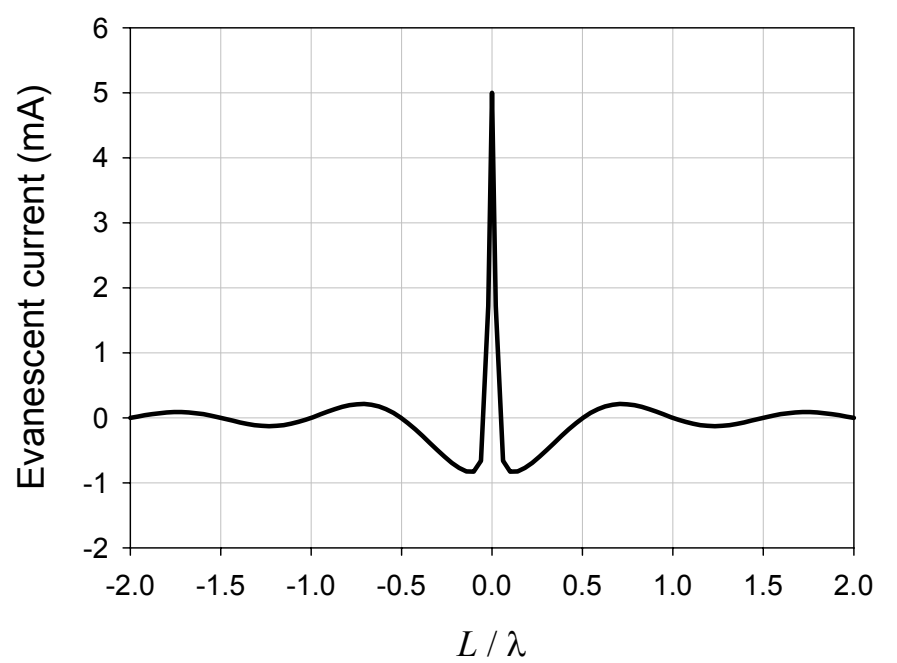

Figure 4.5 Evanescent current obtained in an infinite conducting wire of radius $a=0.5 \mathrm{~mm}$, when fed by a magnetic frill source of radius $b=5 \mathrm{~mm}$. 


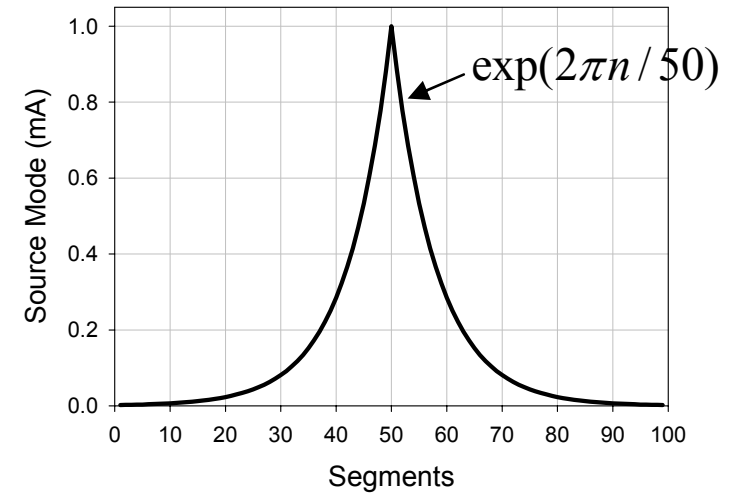

(a) Source at segment 50.

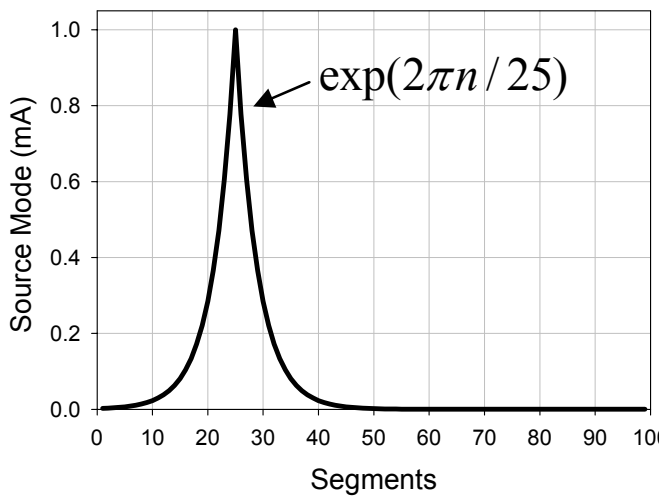

(b) Source at segment 25.

Figure 4.6 Exponential source mode for two dipoles of length $L=0.5 \mathrm{~m}$ fed at different positions.

Figure 4.7 shows the approximation at $280 \mathrm{MHz}$ for the imaginary part of the total current of the centre fed dipole, and the dipole fed at segment 25, when the exponential source mode (SM) is added to the series of characteristic modes. It can be observed that the addition of the source mode improves the convergence of the imaginary part of the series. The approximation for the real part of the current has not been depicted since it is not affected by the addition of the source mode.

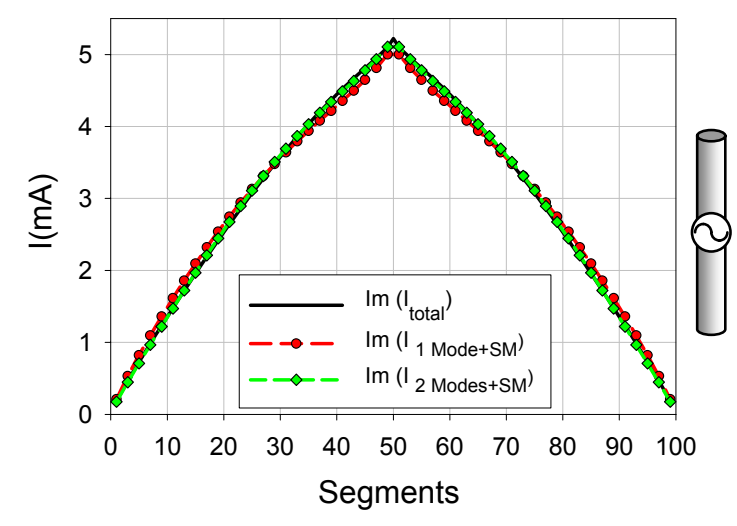

(a) Source at segment 50.

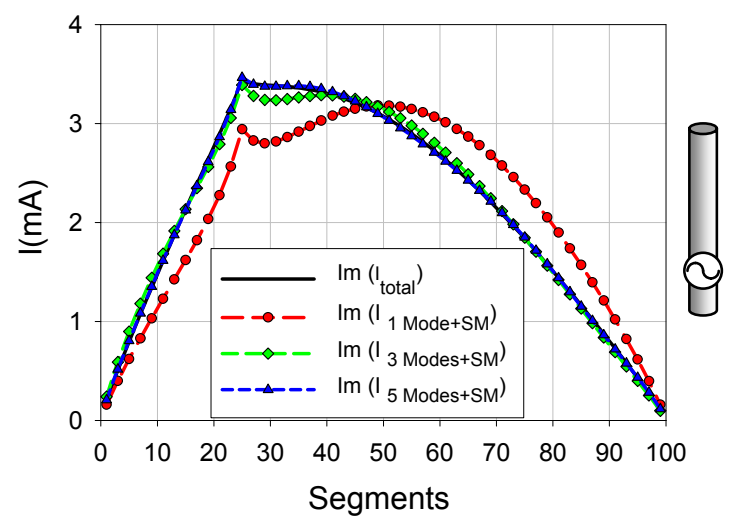

(b) Source at segment 25.

Figure 4.7 Approximation at $280 \mathrm{MHz}$ for the imaginary part of the total current of the centre fed dipole, and a dipole fed at segment 25 , when the exponential source mode is added to the series of characteristic modes.

Results exhibited in Table 4.3 confirm that just using two even characteristic modes plus the source mode, a relative quadratic error less than $1 \%$ is obtained at $280 \mathrm{MHz}$, for the imaginary part of the input current of the centre-fed dipole. Likewise, Table 4.4 demonstrates that for the case of the dipole fed at segment 25 , when the exponential source mode is included in the series, a relative quadratic error less than $1 \%$ can be achieved for the imaginary part of the input current at $280 \mathrm{MHz}$, considering only five modes. 


\begin{tabular}{|c|c|c|}
\hline $\begin{array}{c}\text { Number of even } \\
\text { modes }\end{array}$ & $\begin{array}{c}\text { \% Relative quadratic error } \\
\text { Real part }\end{array}$ & $\begin{array}{c}\text { \% Relative quadratic error } \\
\text { Imaginary part }\end{array}$ \\
\hline 1 & $1.18 \mathrm{e}-4$ & 2.2107 \\
\hline 2 & $1.57 \mathrm{e}-8$ & 0.3803 \\
\hline
\end{tabular}

Table 4.3. Relative quadratic error computed at $280 \mathrm{MHz}$ for the imaginary part of the input current of the centre fed dipole, when an exponential source mode is added to the series of characteristic modes.

\begin{tabular}{|c|c|c|}
\hline $\begin{array}{c}\text { Number of even } \\
\text { modes }\end{array}$ & $\begin{array}{c}\text { \% Relative quadratic error } \\
\text { Real part }\end{array}$ & $\begin{array}{c}\text { \% Relative quadratic error } \\
\text { Imaginary part }\end{array}$ \\
\hline 1 & 0.038 & 15.33 \\
\hline 2 & $1.22 \mathrm{e}-5$ & 2.68 \\
\hline 3 & $6.03 \mathrm{e}-7$ & 2.48 \\
\hline 4 & $1.23 \mathrm{e}-8$ & 1.3 \\
\hline 5 & $1.37 \mathrm{e}-8$ & 0.31 \\
\hline
\end{tabular}

Table 4.4. Relative quadratic error computed at $280 \mathrm{MHz}$ for the imaginary part of the input current the centre dipole fed at segment 25, when an exponential source mode is added to the series of characteristic modes.

However, the exponential source modes illustrated at Figure 4.6 are not only valid to improve the convergence of the series of modes at resonance, but also at other frequencies. Table 4.5 summarizes the relative quadratic error obtained when approximating the input current of the centre-fed dipole at different frequencies, using three, five, and ten even modes, plus the exponential source mode of Figure 4.6 (a). From this table it is extracted that using more than five modes plus the exponential source mode, a relative quadratic error of less than $1 \%$ is achieved at all frequencies, except close to the antiresonce ( $525 \mathrm{MHz}$ ). At the antiresonance the relative error increases, because of the small magnitude exhibited by the input current.

\begin{tabular}{|c|c|c|c|c|c|c|}
\cline { 2 - 7 } \multicolumn{1}{c|}{} & \multicolumn{2}{c|}{3 even modes + SM } & \multicolumn{2}{c|}{5 even modes +SM } & \multicolumn{2}{c|}{10 even modes +SM } \\
\hline Frequencies & $\begin{array}{c}\text { \% RQE } \\
\text { Real part }\end{array}$ & $\begin{array}{c}\text { \% RQE } \\
\text { Imag. part }\end{array}$ & $\begin{array}{c}\text { \% RQE } \\
\text { Real part }\end{array}$ & $\begin{array}{c}\text { \% RQE } \\
\text { Imag. part }\end{array}$ & $\begin{array}{c}\text { \% RQE } \\
\text { Real part }\end{array}$ & $\begin{array}{c}\text { \% RQE } \\
\text { Imag. part }\end{array}$ \\
\hline $300 \mathrm{MHz}$ & $3.70 \mathrm{e}-8$ & 0.22 & $3.13 \mathrm{e}-8$ & 0.20 & $1.48 \mathrm{e}-8$ & 0.08 \\
\hline $400 \mathrm{MHz}$ & $1.00 \mathrm{e}-6$ & 0.94 & $9.00 \mathrm{e}-7$ & 0.79 & $4.44 \mathrm{e}-7$ & 0.37 \\
\hline $500 \mathrm{MHz}$ & $4.21 \mathrm{e}-6$ & 7.98 & $3.85 \mathrm{e}-6$ & 6.37 & $1.93 \mathrm{e}-6$ & 3.05 \\
\hline $600 \mathrm{MHz}$ & $1.04 \mathrm{e}-5$ & 3.92 & $9.80 \mathrm{e}-6$ & 3.00 & $4.98 \mathrm{e}-6$ & 1.46 \\
\hline $700 \mathrm{MHz}$ & $1.70 \mathrm{e}-5$ & 1.83 & $1.71 \mathrm{e}-5$ & 1.31 & $8.81 \mathrm{e}-6$ & 0.65 \\
\hline $800 \mathrm{MHz}$ & $9.34 \mathrm{e}-6$ & 0.95 & $1.13 \mathrm{e}-5$ & 0.62 & $5.81 \mathrm{e}-6$ & 0.31 \\
\hline $900 \mathrm{MHz}$ & $7.97 \mathrm{e}-7$ & 1.61 & $3.10 \mathrm{e}-6$ & 0.93 & $1.60 \mathrm{e}-6$ & 0.46 \\
\hline
\end{tabular}

Table 4.5. Relative quadratic error obtained when approximating the input current of the centre fed dipole at different frequencies, using three, five and ten even modes plus the exponential source mode. 


\subsection{Frequency independent basis functions}

As it was commented in the introduction of this chapter, one of the drawbacks of using characteristic modes as entire domain-basis functions in the MoM formulation is that characteristic modes depend upon frequency. This dependency should be taken into account to get to accurate results, especially at the highest frequencies. Thus, characteristic modes need to be obtained and stored for each frequency under analysis. Because of this limitation, it would be very advantageous to obtain a set of basis functions that would not depend upon frequency and would comprise the behaviour of characteristic modes in the entire frequency band of interest. This can be accomplished using the Singular Value Decomposition (SVD) as proposed in [89].

The Singular Value Decomposition (SVD) [47] is a classical technique used to factorize any nonzero matrix $[W]_{m \times n}$ with $m>n$, and rank $r$, as

$$
[W]=[U][\Sigma][V]^{\prime}
$$

where $[U]_{m \times m}$ and $[V]_{n \times n}$ are orthogonal matrices, and $[\Sigma]_{m \times n}$ is a non-singular diagonal matrix, with diagonal entries $\sigma_{i}$

$$
[\Sigma]_{m \times n}=\left[\begin{array}{cc}
D & 0 \\
0 & 0
\end{array}\right], \quad \text { with } \quad[D]=\left[\begin{array}{ccc}
\sigma_{1} & \cdots & 0 \\
\vdots & \ddots & \vdots \\
0 & \cdots & \sigma_{r}
\end{array}\right]
$$

The diagonal elements $\sigma_{n}$, that are called the singular values of $[\mathrm{W}]$, are always positive and sorted so that $\sigma_{1} \geq \sigma_{2} \geq \cdots \geq \sigma_{r} \geq 0$. The vector columns $\left\{v_{1}, v_{2}, \cdots, v_{n}\right\}$ of $[V]$, are the right singular vectors of $[W]$, and the vector columns $\left\{u_{1}, u_{2}, \cdots, u_{m}\right\}$ of $[U]$, are the left singular vectors of $[W]$.

If $[W]$ is a matrix with rank $r$, the right and left singular vectors will present the following properties

$\left\{u_{1}, u_{2}, \cdots, u_{r}\right\}$ forms an orthonormal basis for the vector columns of $[W]$.

$\left\{v_{1}, v_{2}, \cdots, v_{r}\right\}$ forms and orthonormal basis for the vector rows of $[W]$.

Hence, SVD performs a linear transformation of a matrix $[\mathrm{W}]$, whose columns/rows are linearly dependent vectors, into a reduced size matrix $[U] /[V]$, whose columns/rows are linearly independent vectors that comprise the behaviour of all the vector columns/rows in $[W]$. 
But, let us see how SVD can be used to obtain a set of frequency independent characteristic modes (FICM), from Harrington's characteristic modes. The overall procedure to generate the set of FICM is described below, and summarized in Figure 4.8.

\section{General procedure to generate the set of FICM}

1. Choose the number of characteristic modes, say $M$, that are going to be used to expand the total current.

2. Calculate these $M$ characteristic modes at $N$ uniformly spaced frequencies over the band of interest.

3. Form a new matrix $[W]$ whose columns are the $M \times N$ modes obtained in step 2, plus the exponential source mode proposed in section 4.4 to accelerate convergence.

4. Then, apply singular value decomposition (SVD) to matrix $W$, to get a new matrix $U$, whose columns are the left singular vectors that form an orthonormal basis that span the subspace formed by the vector columns of $W$.

5. Take the columns of $U$ with larger associated singular values $\left(\sigma_{n}\right)$, say $P$ in number, to form the new set of frequency independent basis functions.

6. Finally, use these $P$ FICM at every frequency to get to a compressed MoM equation of dimension $P X P$ that can be solved by direct inversion.

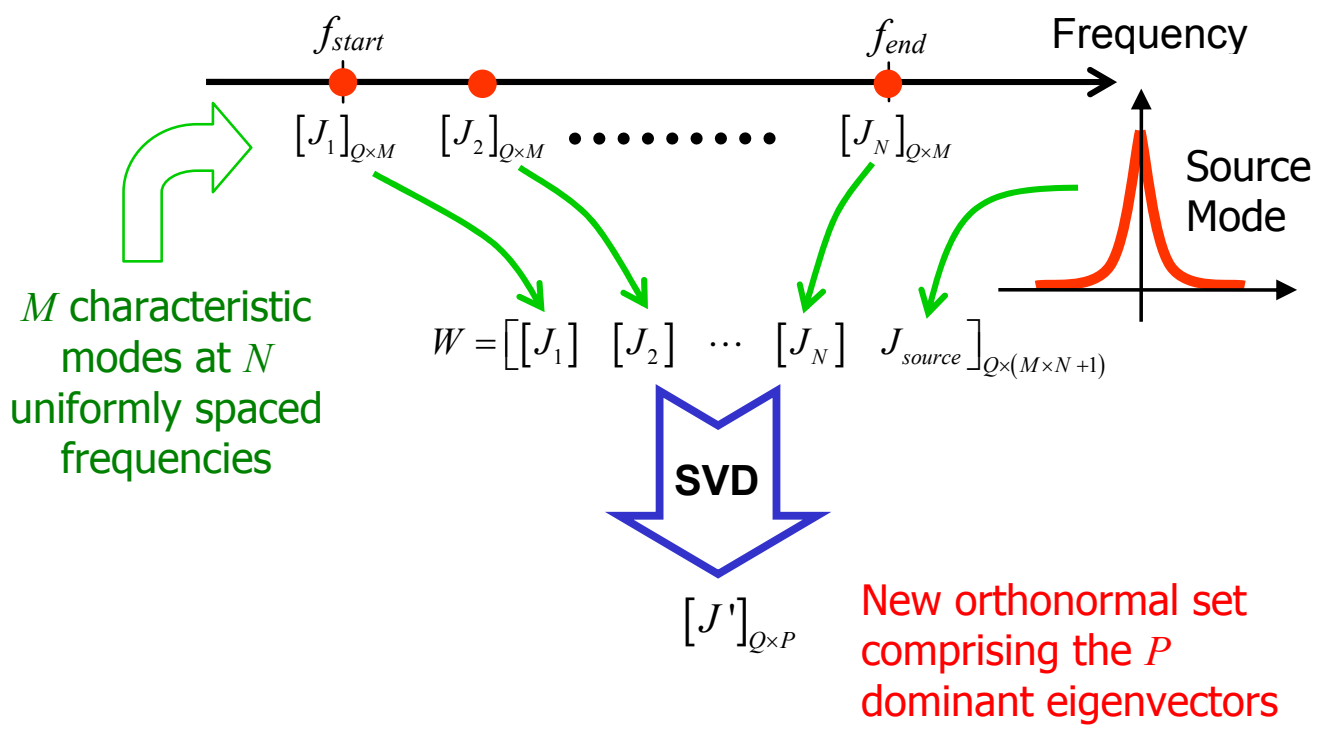

Figure 4.8 Procedure used to generate the set of $P$ FICM. 
To illustrate the procedure described above, and to verify that the exponential source mode presented in section 4.4 is valid for any type of wire antenna, the input impedance of the square wire loop shown in Figure 4.9 is going to be approximated using a set of FICM. This square loop has already been analyzed in section 3.4.1 of Chapter 3. It has a perimeter of $0.916 \mathrm{~m}$ that is divided in 48 segments. The wire radius is $0.5 \mathrm{~mm}$, and the structure is fed at the centre of one arm (segment 24) by a delta gap source.

Figure 4.10 compares the total current of the square loop at $360 \mathrm{MHz}$ (first resonance), with its modal approximation which uses a few even characteristic modes for the expansion. As can be seen, the real part converges just using one even mode, whereas for the imaginary part more than seven even modes are needed to converge to the exact solution. Once the poor convergence of the series of characteristic modes for the square loop has been demonstrated, let us apply to this loop the improvements described in the preceding sections.

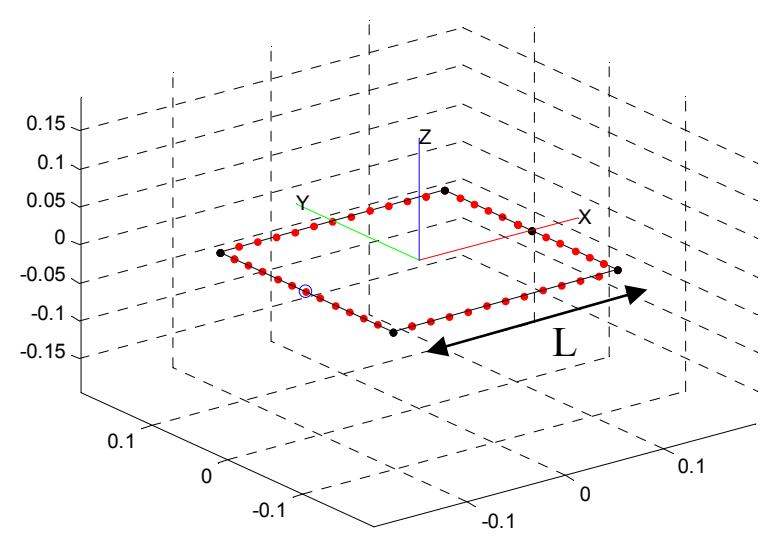

Figure 4.9 Square loop of perimeter $0.916 \mathrm{~m}$ and wire radius $r=0.5 \mathrm{~mm}$.

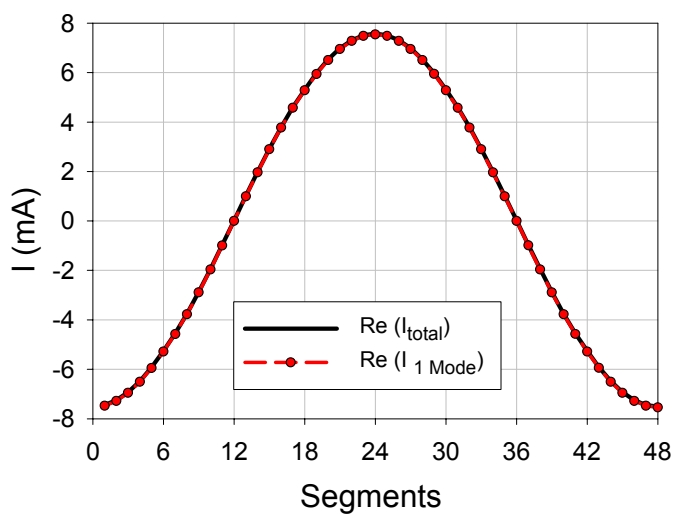

(a) Real part of the current.

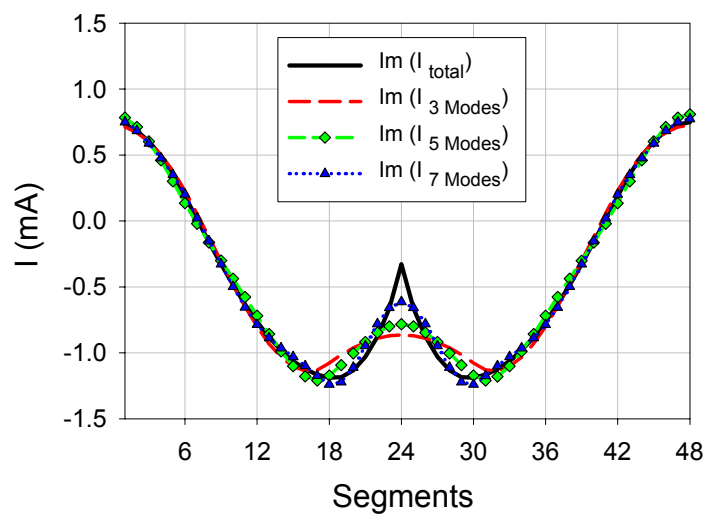

(b) Imaginary part

Figure 4.10 Comparison of the total current the square loop fed at segment 24, and the current obtained by modal expansion at $360 \mathrm{MHz}$. 
To apply the procedure for the generation of the set of FICM, the number of modes considered for the expansion of the current is $M=7$. The current distribution of these first seven modes (even and odd) of the square loop, was depicted in section 3.4 of Chapter 3. Then, these $M$ modes are computed numerically at $N=6$ uniformly spaced frequency points over the band of interest, that ranges from $20 \mathrm{MHz}$ to $1 \mathrm{GHz}$. Next, the 42 resultant modes plus the exponential source mode added to fasten the convergence, are arranged as column vectors to form matrix $W$ of size $48 \times 43$. Figure 4.11 shows the exponential source mode employed for this loop fed at segment 24.

Just to finish with the procedure, SVD is applied to matrix $W$ to get the new set of $P=15$ FICM. Figure 4.12 shows the current distribution of the first eight modes of the new set of FICM. Most of these new modes present a current distribution with increasing oscillatory nature. However, it is worth highlighting that, among these modes there is a uniform current mode (FICM n03), and also a source mode (FICM $\mathrm{n}^{06}$ ) which resembles very much the residual current mode of Figure 4.3.

Finally, to validate the accuracy of the proposed method, Figure 4.12 compares the input impedance of the square loop computed using MoM with sub-sectional pulse basis functions, with the one obtained by modal expansion, using the set of 15 FICM at all frequencies. It can be observed that both results are in close agreement. For the shake of completeness, Table 4.6 sketches the relative quadratic error obtained at several frequencies for this input impedance, when the 15 FICM are used for the approximation. Surprisingly, the error keeps below $1 \%$ within the whole band of interest. Therefore, these results demonstrate that the FICM derived from characteristic modes plus the source mode, can be used as an effective set of wide-band basis functions, for the analysis of an antenna in a wide frequency range.

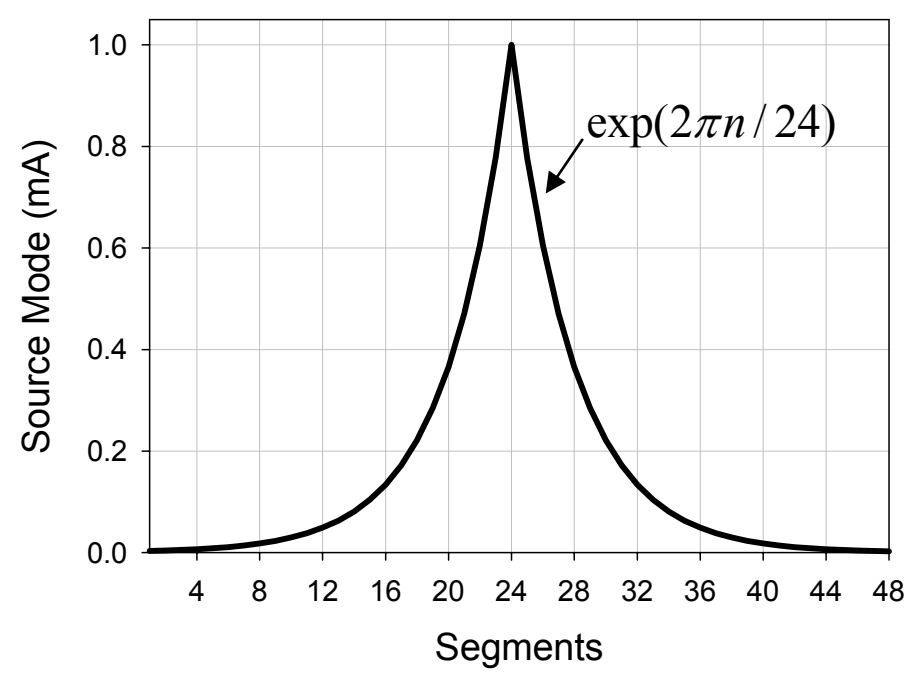

Figure 4.11 Exponential source mode for the square loop fed at segment 24. 


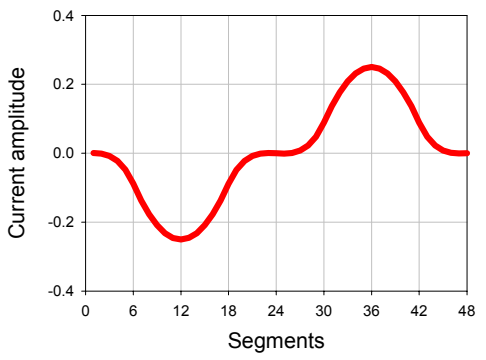

(a) FICM no 1

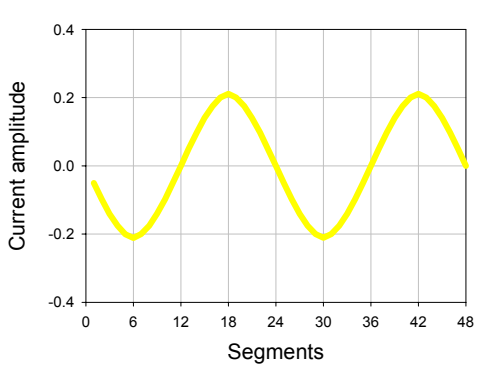

(d) FICM n 4

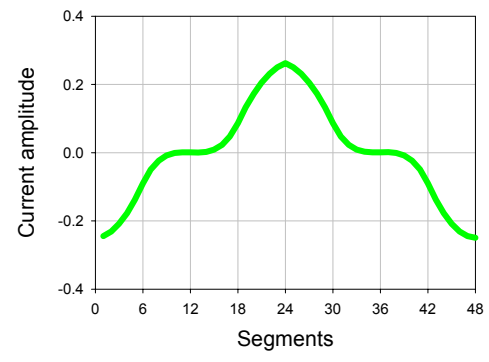

(b) FICM n²

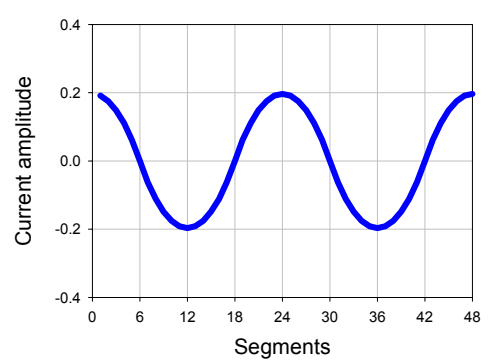

(e) FICM n 5

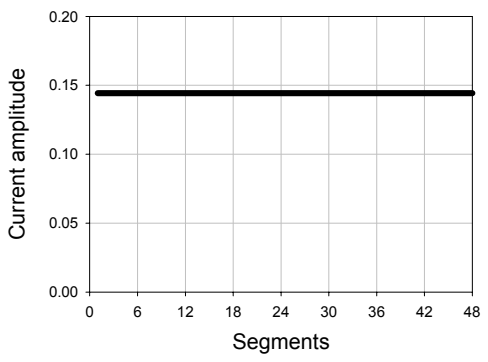

(c) FICM n 3

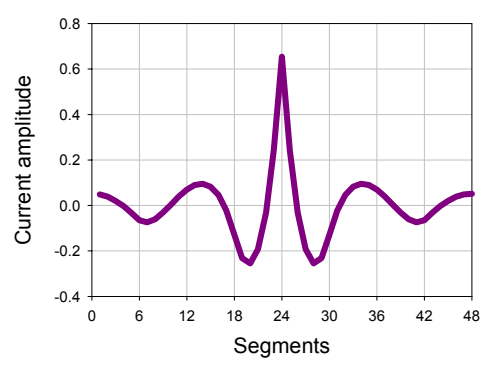

(f) $\mathrm{FICM} \mathrm{n}{ }^{\circ} 6$

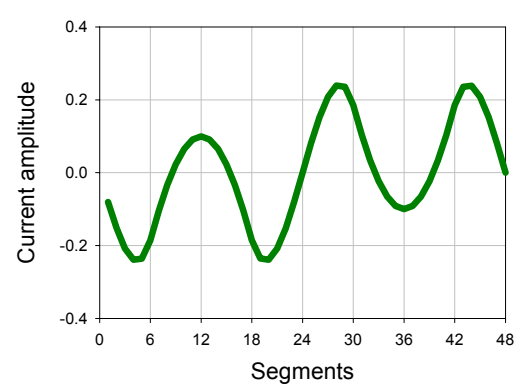

(g) FICM no 7

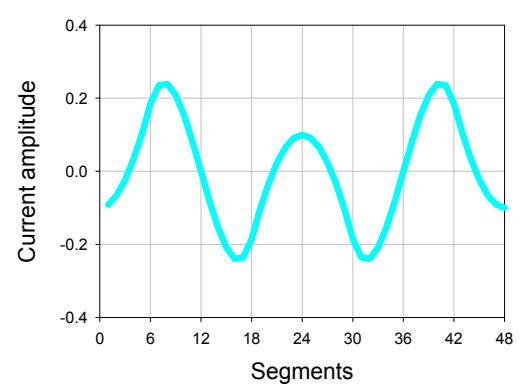

(h) FICM n 8

Figure 4.12 Current distribution of the first eight frequency independent characteristic modes.

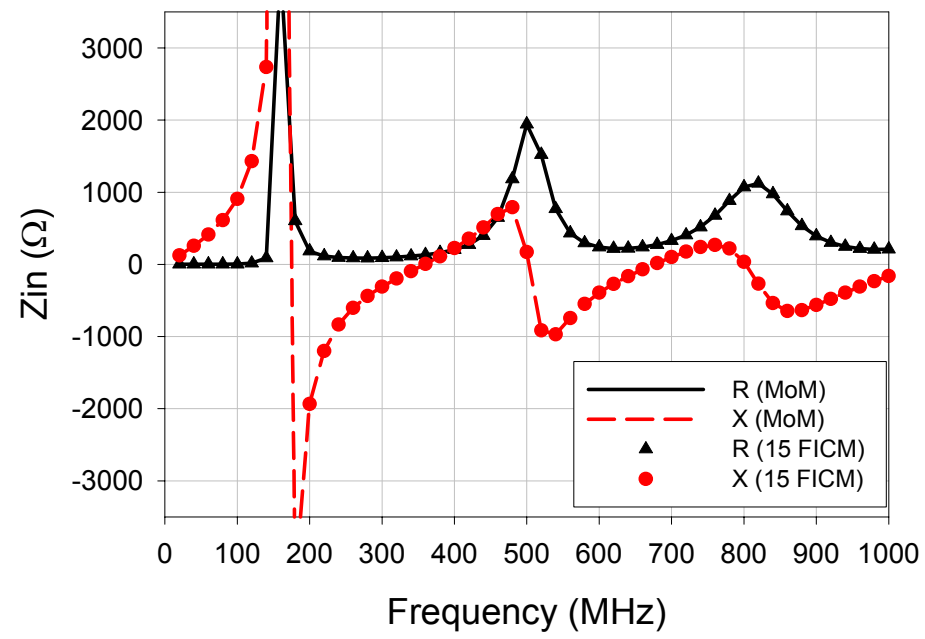

Figure 4.13 Input impedance of the square loop fed at segment 24 computed using MoM with pulse basis functions, and computed using the set of 15 FICM. 


\begin{tabular}{|c|c|c|}
\hline Frequency & $\begin{array}{c}\text { \% Relative quadratic error } \\
\text { Real part of } Z_{\text {in }}\end{array}$ & $\begin{array}{c}\text { \% Relative quadratic error } \\
\text { Imaginary part of } Z_{\text {in }}\end{array}$ \\
\hline $20 \mathrm{MHz}$ & $4.65 \mathrm{e}-5$ & 0.0012 \\
\hline $100 \mathrm{MHz}$ & 0.001 & 0.042 \\
\hline $360 \mathrm{MHz}$ & 0.007 & 0.370 \\
\hline $600 \mathrm{MHz}$ & 0.003 & 0.079 \\
\hline $1 \mathrm{GHz}$ & 0.265 & 0.051 \\
\hline
\end{tabular}

Table 4.6. Relative quadratic error obtained at different frequencies when approximating the input impedance of the square loop fed at segment 24, using 15 FICM.

\subsection{Convergence of the series of characteristic modes for patch antennas.}

Let us verify now that for the case of planar antennas, the series of characteristic modes also presents slow convergence when it is used to approximate the imaginary part of the input current. For this purpose, the narrow metallic strip shown in Figure 4.14, is going to be considered. The strip is in free space, and its dimensions are $L=6 \mathrm{~cm}$ and $W=0.5 \mathrm{~cm}$.

The generalized impedance matrix of this structure is obtained using the MoM in combination with RWG edge elements (see Appendix 2 for more details). First, the surface of the antenna is divided into separate triangles as shown in Figure 4.14. Each pair of triangles having a common edge constitutes the corresponding RWG edge element [45]. The surface electric current density on the antenna surface (a vector) is a sum of the contributions over all the edge elements, with unknown coefficients. These coefficients are found from classical moment equations.

The first resonance of this structure takes place when the length of the strip $L$ is approximately $\lambda / 2$. Figure 4.15 shows the real and imaginary parts of the current on surface of the strip close to first resonance (at $2.4 \mathrm{GHz}$ ) when the antenna is fed with a delta gap source placed at edge 50 . This total current has been obtained by direct inversion of the 99x99 impedance matrix. As observed, since the strip is very narrow, the current flows longitudinally at both the real and imaginary parts.

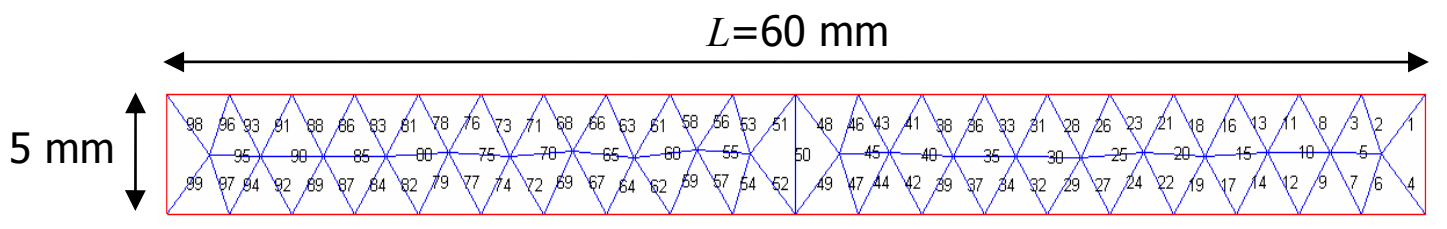

Figure 4.14 Metallic strip at free space. 


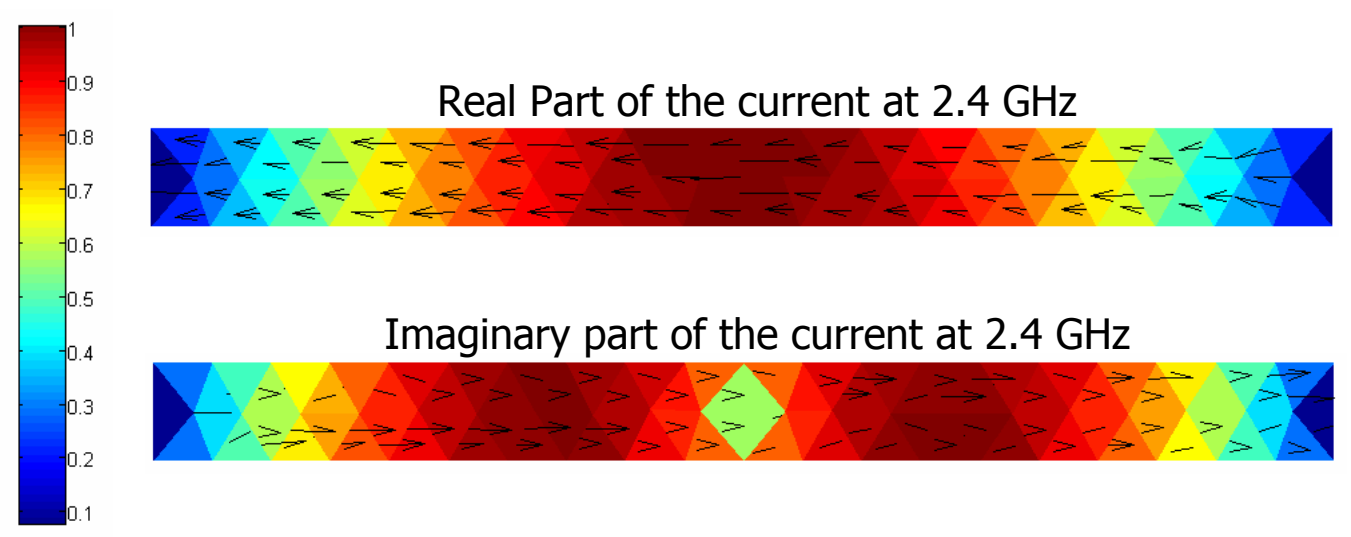

Figure 4.15 Real and imaginary parts for the current on the surface of the metallic strip when it is fed at edge 50 .

Now, let us approximate the total current on the surface of the strip, using an expansion of characteristic modes. For the sake of example, Figure 4.16 presents an approximation using 15 characteristic modes, for both the real and imaginary parts of the current at $2.4 \mathrm{GHz}$, when the strip is fed at edge 50 . When results in Figure 4.15 and Figure 4.16 are compared, it is noticeable that the approximation at the feeding point for the imaginary part of the current is not accurate enough.

Figure 4.17 compares the actual total current and the current approximated with 15 modes at $2.4 \mathrm{GHz}$, this time using a linear representation of the current amplitude along the 99 internal edges. Like in wire antennas, the modal expansion yields a very good approximation for the real part of the total current, whereas the approximation for the imaginary part exhibits poor convergence, with maximum error concentrated at the feeding point (edge number 50). Observe the current at edges multiple of five $(5,10,15,20,25 \ldots)$ is nearly zero. This is because there is no transverse current flow, so currents passing through edges which are a multiple of five are very small. See Figure 4.14 to check the position of these edges.

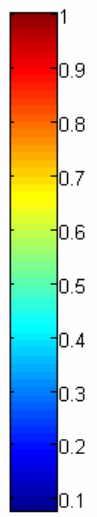

Real part of the current approximated with 15 modes at $2.4 \mathrm{GHz}$

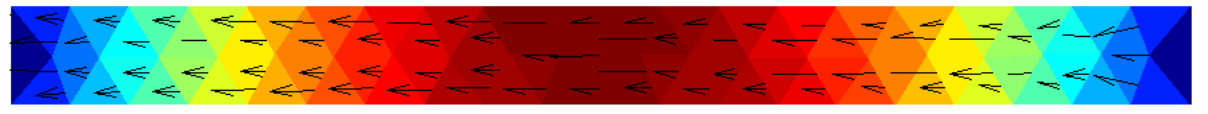

Imaginary part of the current approximated with 15 modes at $2.4 \mathrm{GHz}$

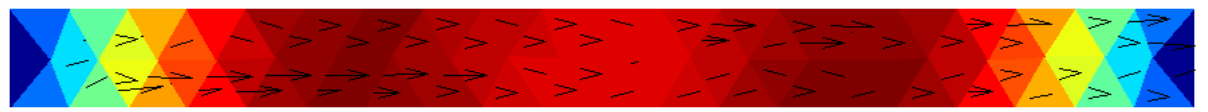

Figure 4.16 Real and imaginary parts for the current approximated with 15 characteristic modes, when the strip is fed at edge 50. 


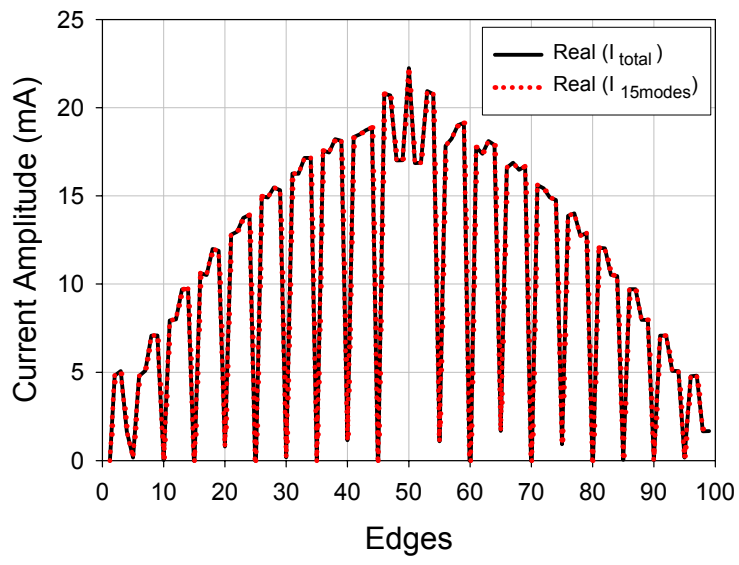

(a) Real part

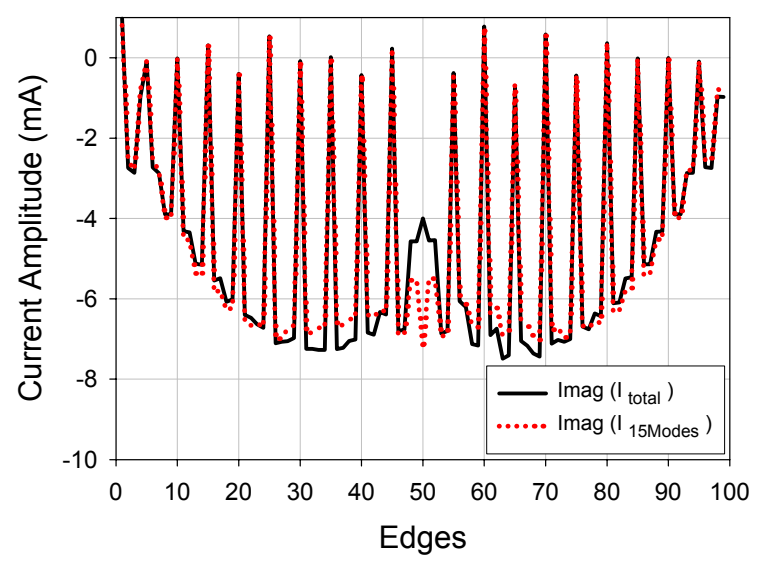

(b) Imaginary part

Figure 4.17 Actual total current and current approximated with 15 characteristic modes at $2.4 \mathrm{GHz}$ for the metallic strip fed at edge 50 .

As the number of modes used for the expansion increases, the convergence improves, but in a very slow way. Table 4.7 summarizes the relative quadratic error computed at $2.4 \mathrm{GHz}$ when the total input current of the centre fed strip is approximated by an expansion with variable number of modes. Unfortunately, although the real part of the input current converges very fast, the relative quadratic error for the imaginary component of the current is bigger than $1 \%$, even using an expansion of 90 modes. Once again, the explanation for this slow convergence of the imaginary part of the current is the presence of the delta gap source over the structure, which creates an evanescent current that can not be modelled properly by the series of characteristic modes.

Following the approach already presented for the wire antenna class, a source mode may be included in the series of characteristic modes in order to fasten its convergence. But, which is the optimum shape the source mode may have for planar antennas?

\begin{tabular}{|c|c|c|}
\hline $\begin{array}{c}\text { Number of } \\
\text { modes }\end{array}$ & $\begin{array}{c}\text { \% Relative quadratic error } \\
\text { Real part }\end{array}$ & $\begin{array}{c}\text { \% Relative quadratic error } \\
\text { Imaginary part }\end{array}$ \\
\hline 3 & $1.48 \mathrm{e}-3$ & 134.95 \\
\hline 5 & $1.48 \mathrm{e}-3$ & 134.95 \\
\hline 10 & $4.22 \mathrm{e}-8$ & 98.07 \\
\hline 15 & $1.67 \mathrm{e}-10$ & 81.10 \\
\hline 20 & $1.05 \mathrm{e}-12$ & 83.33 \\
\hline 50 & $3.35 \mathrm{e}-13$ & 18.01 \\
\hline 75 & $3.35 \mathrm{e}-13$ & 5.15 \\
\hline 90 & $3.35 \mathrm{e}-13$ & 1.39 \\
\hline
\end{tabular}

Table 4.7. Relative quadratic error computed at first resonance $(2.4 \mathrm{GHz})$ when the total input current of the centre fed strip is approximated by an expansion with variable number of modes. 
A first approximation for this source mode can be observed in Figure 4.18, which shows the residue that is left after subtracting to the actual total current at $2.4 \mathrm{GHz}$, the approximation yield by the expansion of 15 characteristic modes. From this linear representation it is extracted that this residue is very similar to that obtained in Figure 4.3 for the centre fed wire dipole. Once more, due to the presence of the delta gap source, there exist an evanescent current along the strip, with a peak value just at the feeding edge, and with a fading profile away from it.

A planar strip can be considered the result of flattening a circular cylinder, therefore, the analytical demonstration for the existence of an evanescent current mode linked to the source presented for wire antennas, is also valid for a planar strip.

The source mode in Figure 4.18 could be approximated by an exponential function, which has been demonstrated to provide excellent results for wire antennas. However, in planar antennas the projection of an exponential source mode over the meshing of the structure could be very complex in general and quite time-consuming.

A straightforward solution could consist in using a very simplified source mode, like the one sketched in Figure 4.19. This delta gap source mode can be easily implemented by means of a vector with zeros at all edges, except at the feeding edge.
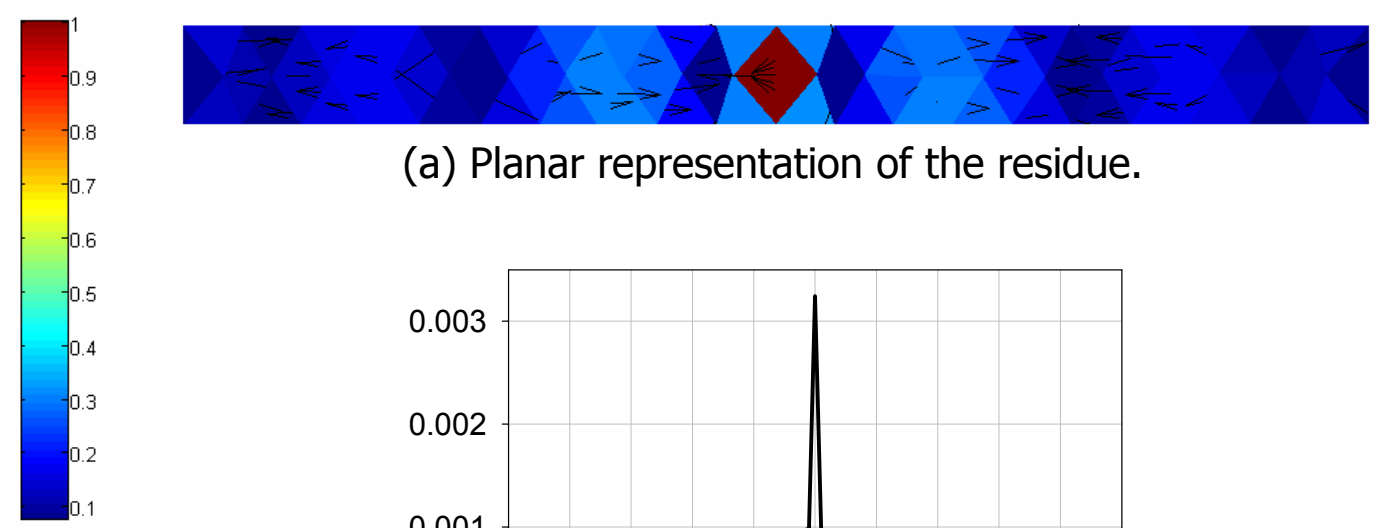

(a) Planar representation of the residue.

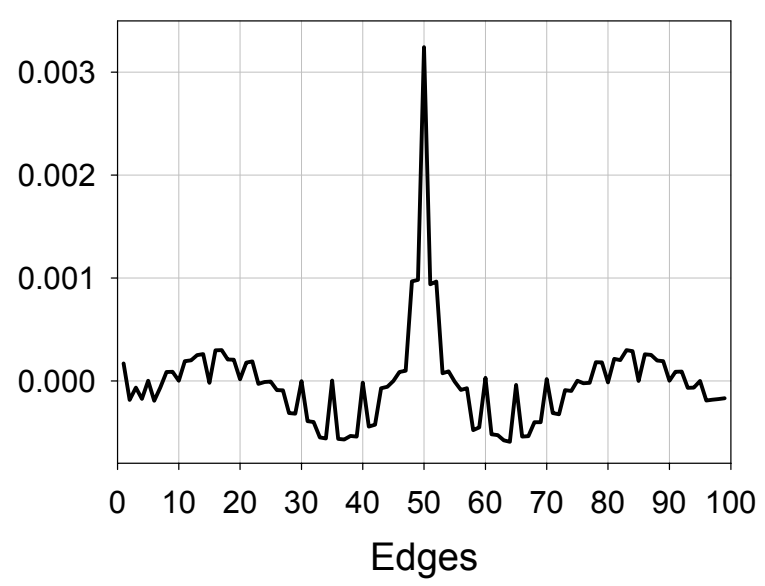

(b) Linear representation of the residue.

Figure 4.18 Residue that is left after subtracting to the actual total current at $2.4 \mathrm{GHz}$, the approximation yield by an expansion of 15 characteristic modes. 


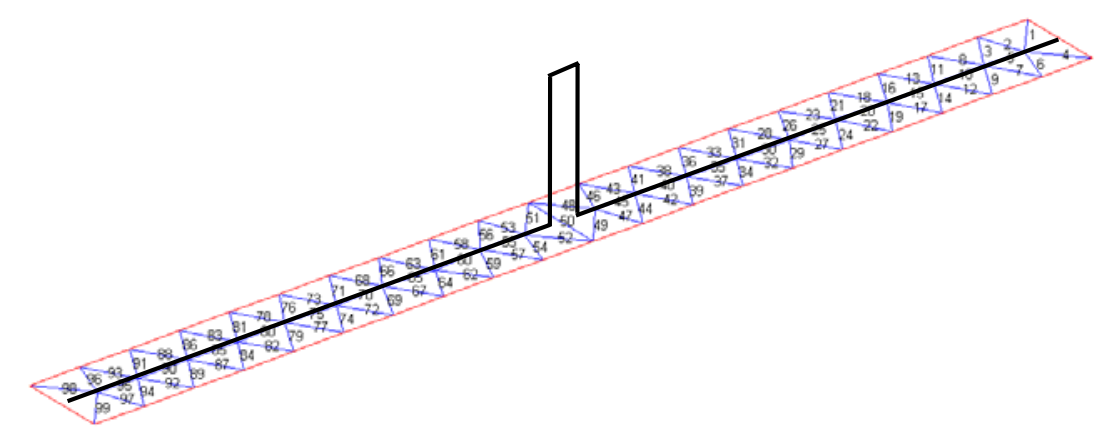

Figure 4.19 Simplified delta gap source mode for the metallic strip fed at edge 50.

Table 4.8 gathers the relative quadratic error computed at $2.4 \mathrm{GHz}$ when the total input current of the centre-fed strip is approximated by an expansion with variable number of modes plus the simplified delta-gap source mode. As observed, the relative quadratic error for the real part of the input current is still very small. As expected, the error for the imaginary part has been reduced, especially when using small number of modes. However, even including this simplified source mode, convergence is still very slow.

Although previous results demonstrate that the addition a the delta gap source mode accelerates the converge of the series of modes, the relative quadratic error for the imaginary part of the input current at first resonance is still $1.28 \%$ when 90 modes are considered for the expansion of the current.

\begin{tabular}{|c|c|c|}
\hline $\begin{array}{c}\text { Number of } \\
\text { modes }\end{array}$ & $\begin{array}{c}\text { \% Relative quadratic error } \\
\text { Real part }\end{array}$ & $\begin{array}{c}\text { \% Relative quadratic error } \\
\text { Imaginary part }\end{array}$ \\
\hline 3 & $1.49 \mathrm{e}-3$ & 71.67 \\
\hline 5 & $1.49 \mathrm{e}-3$ & 71.67 \\
\hline 10 & $4.16 \mathrm{e}-8$ & 38.42 \\
\hline 15 & $4.27 \mathrm{e}-11$ & 24.88 \\
\hline 20 & $1.08 \mathrm{e}-12$ & 24.83 \\
\hline 50 & $6.55 \mathrm{e}-13$ & 12.75 \\
\hline 75 & $5.43 \mathrm{e}-13$ & 4.52 \\
\hline 90 & $5.59 \mathrm{e}-13$ & 1.28 \\
\hline
\end{tabular}

Table 4.8. Relative quadratic error computed at first resonance $(2.4 \mathrm{GHz})$ when the input current of the centre fed strip is approximated by an expansion with variable number of modes plus the delta gap source mode.

Some numerical experiments have been carried out adding different approximations of the residual source mode in Figure 4.18 to the series of modes. Triangular-shaped source modes, as well as exponential source modes have been found to provide better results than the simplified delta gap source mode. However, these source modes have been created to fit the particular 
meshing of this strip, so they do not represent a standard solution for the convergence problem of characteristic modes in planar antennas.

Of course, if the source mode obtained in Figure 4.18 were added to the series of modes, the convergence for the imaginary part of the current at resonance would be very fast. But, would this residue obtained at $2.4 \mathrm{GHz}$ improve the convergence at the rest of frequencies too?

Table 4.9 verifies that the relative quadratic error for the imaginary part of the input current is very much reduced when the residual source mode computed at $2.4 \mathrm{GHz}$ is added to the series of 15 modes at different frequencies.

therefore, the question that arises now is whether, it is possible to obtain a set of Frequency Independent Characteristic Modes (FICM) valid in a wide of frequencies including the effect of the source mode or not.

As explained in Section 4.5, using a SVD decomposition, a set of characteristic modes at different frequencies plus the source mode, can be transformed in a reduced set of FICM that expands the current very accurately in a wide range of frequencies. Next, the procedure described in Section 4.5 is going to be applied to the modes of the metallic strip.

Firstly, Figure 4.20 depicts the normalized current distribution for the first modes of the strip at $2.4 \mathrm{GHz}$. The left side of Figure 4.20 shows the distribution of the modal currents along the strip. On the right side, linear representations of the current amplitude of modes along the 99 internal edges are presented. As the strip is relatively narrow, all modes exhibit longitudinal current flow. Mode $J_{1}$ is the fundamental mode, and as well as modes $J_{0}, J_{3}$ and $J_{4}$, it presents even current distribution. Mode $J_{2}$ is the only mode with odd current distribution. Note in all modes the current at edges multiple of five is very small.

\begin{tabular}{|c|c|c|}
\cline { 2 - 3 } \multicolumn{1}{c|}{} & \multicolumn{2}{c|}{ Expansion with 15 modes + Residual Source Mode } \\
\hline Frequencies & $\begin{array}{c}\text { \% Relative Quadratic Error } \\
\text { Real part }\end{array}$ & $\begin{array}{c}\text { \% Relative Quadratic Error } \\
\text { Imag. part }\end{array}$ \\
\hline $1.4 \mathrm{GHz}$ & $1.78 \mathrm{e}-9$ & 3.87 \\
\hline $2.4 \mathrm{GHz}$ & $5.59 \mathrm{e}-13$ & $3.33 \mathrm{e}-12$ \\
\hline $3.4 \mathrm{GHz}$ & $9.03 \mathrm{e}-11$ & 0.0312 \\
\hline $4.4 \mathrm{GHz}$ & $3.12 \mathrm{e}-9$ & 0.023 \\
\hline $5.4 \mathrm{GHz}$ & $4.78 \mathrm{e}-6$ & 0.16 \\
\hline $6.4 \mathrm{GHz}$ & $2.78 \mathrm{e}-6$ & 0.12 \\
\hline $7.4 \mathrm{GHz}$ & $2.45 \mathrm{e}-6$ & 1.04 \\
\hline $8.4 \mathrm{GHz}$ & $2.81 \mathrm{e}-5$ & 1.17 \\
\hline
\end{tabular}

Table 4.9. Relative quadratic error computed at different frequencies when the input current of the centre fed strip is approximated by an expansion with variable number of modes plus the residual source mode of Figure 4.18. 


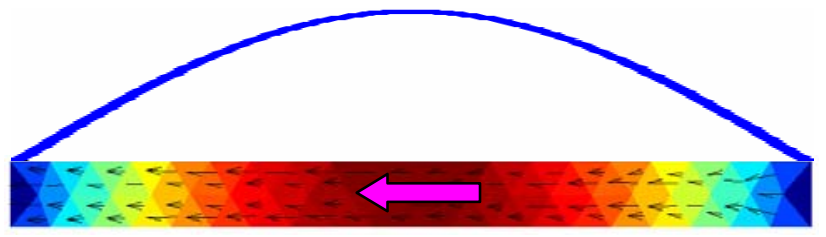

(a) Mode $J_{l}$

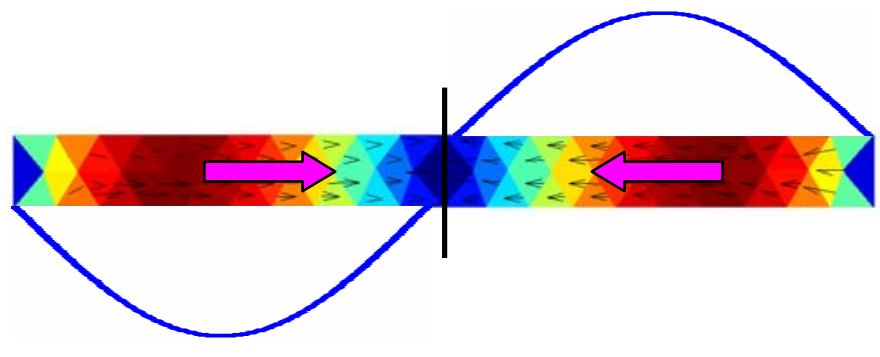

(b) Mode $J_{2}$

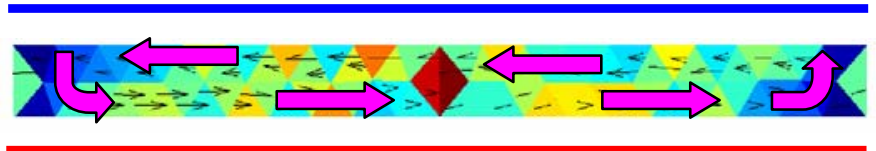

(c) Mode $J_{0}$

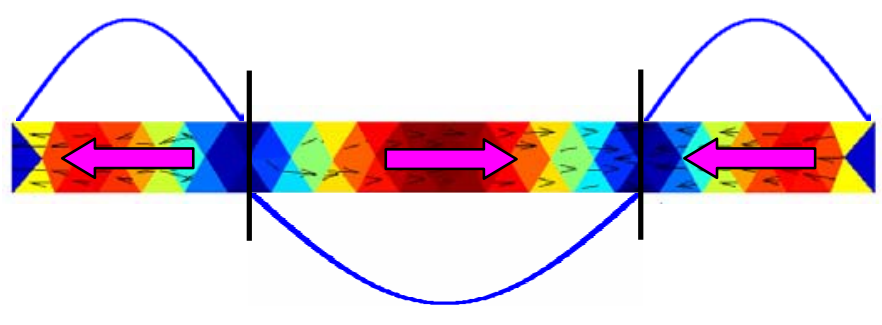

(d) Mode $J_{3}$

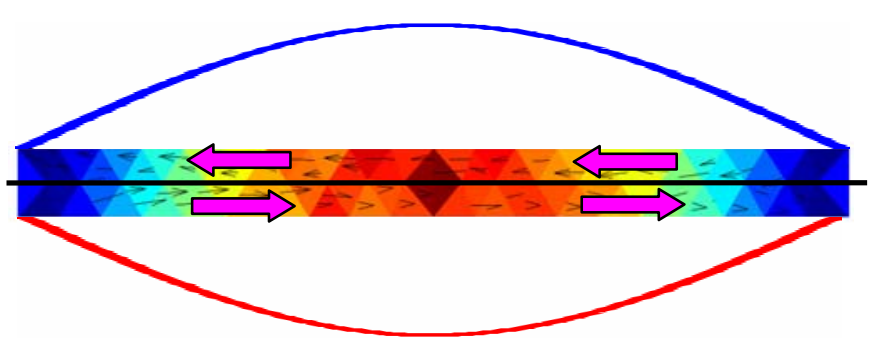

(e) Mode $J_{4}$

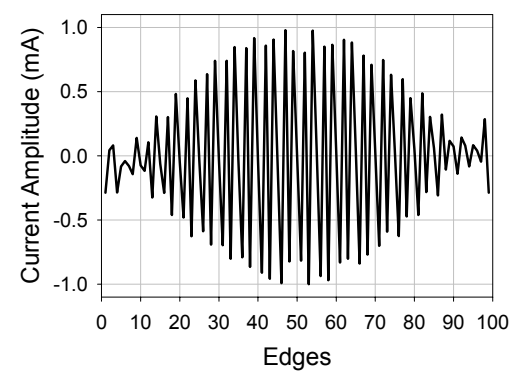

Figure 4.20 Normalized current distribution of the modes of the metallic strip at first resonance $(2.4 \mathrm{GHz})$. 
Figure 4.21 presents characteristic angle variation with frequency for the first modes of the metallic strip. Mode $J_{0}$ is a special non-resonant mode, with inductive behaviour at every frequency. Modes $J_{1}, J_{2}$ and $J_{3}$ resonate at $2.4 \mathrm{GHz}$, $4.8 \mathrm{GHz}$ and $7.2 \mathrm{GHz}$, respectively, while mode $J_{4}$ resonates out of the studied frequency band.

As a general rule, for generating a set of FICM valid at first resonance and nearby frequencies, at least three modes need to be calculated at each frequency selected for the SVD decomposition. If the set of FICM is going to be used close to the second resonance, at least seven modes should be considered. In this case, the number of modes chosen for expanding the current is $M=5$. These five modes correspond with those depicted in Figure 4.20. As shown in Figure 4.21 only three modes resonate in the analyzed frequency band, so the choice of $M=5$ modes, seems reasonable, as it includes the fundamental mode, the special non-resonant mode, and three higher order modes that may cause the subsequent resonances. Then, the $M$ modes are calculated at $N=6$ uniformly spaced frequencies, from $1 \mathrm{GHz}$ to $7 \mathrm{GHz}$. Next, the resultant 30 modes plus the source mode shown in Figure 4.18 are arranged as columns vectors to form a new matrix $W$ of dimension $99 \times 31$. Finally, a SVD decomposition is applied to matrix $W$ to obtain the new set of $P=15$ FICM. This new modes are column vectors of dimension $99 \times 1$.

To validate the above-mentioned procedure, the current on the surface of the centred fed strip has been computed at different frequencies using the new set of 15 FICM. Table 4.10 presents the relative quadratic error for the input current estimated with these 15 FICM. As observed, the error for the imaginary part is less than $1 \%$ at all frequencies except at the antiresonance $(3.4 \mathrm{GHz})$, where it is a bit higher. This extraordinary improvement on the error for the imaginary part of the current is due to the addition of the residual source mode to the set of modes that has been subjected to the SVD decomposition.

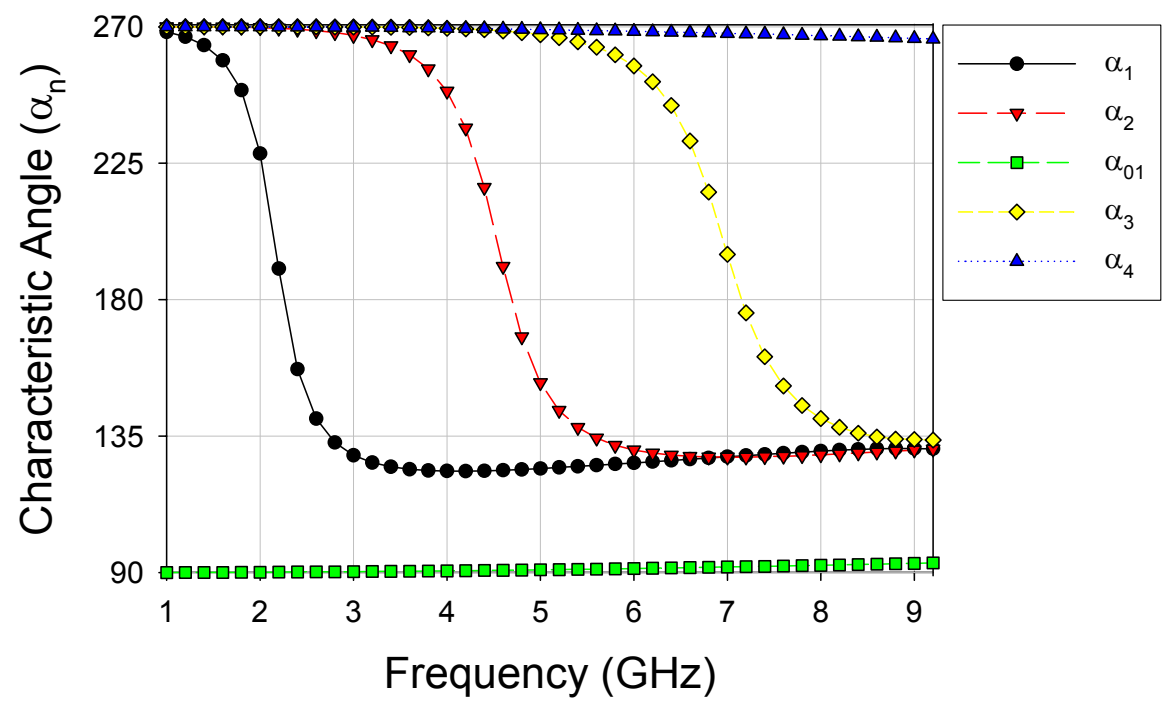

Figure 4.21 Characteristic angle variation with frequency for the first modes of the metallic strip shown in Figure 4.20. 


\begin{tabular}{|c|c|c|}
\cline { 2 - 3 } \multicolumn{1}{c|}{} & \multicolumn{2}{c|}{ Expansion with 15 FICM } \\
\hline Frequencies & $\begin{array}{c}\text { \% Relative Quadratic Error } \\
\text { Real part }\end{array}$ & $\begin{array}{c}\text { \% Relative Quadratic Error } \\
\text { Imaginary part }\end{array}$ \\
\hline $1.4 \mathrm{GHz}$ & 0.0028 & 0.0484 \\
\hline $2.4 \mathrm{GHz}$ & 0.00026 & 0.1765 \\
\hline $3.4 \mathrm{GHz}$ & 0.00176 & 1.470 \\
\hline $4.4 \mathrm{GHz}$ & 0.00243 & 0.1336 \\
\hline $5.4 \mathrm{GHz}$ & 0.00652 & 0.0228 \\
\hline $6.4 \mathrm{GHz}$ & 0.08013 & 0.0686 \\
\hline
\end{tabular}

Table 4.10. Relative quadratic error computed at different frequencies when the input current of the centre fed strip is approximated by an expansion of 15 Frequency Independent Characteristic Modes (FICM).

The SVD approach presented above is worthwhile only when the antenna is to be analyzed is a wide range of frequencies. Note that applying the SVD decomposition, a very good convergence is achieved in a broad range of frequencies, at the expense of calculating the actual total current by direct inversion of the impedance matrix, at least at one frequency, in order to obtain the residual source mode. Also five characteristic modes need to be computed at six frequencies. Nevertheless, the SVD is really suitable to reduce the computational cost when analyzing large arrays with identical wideband radiating elements.

It must be pointed out that although the procedure presented in this work for obtaining the source mode is not very smart, it constitutes a standard solution, as it does not depend on the meshing of the structure. Note a residual source mode calculated by subtraction, can be found for any planar antenna problem. Generally, as demonstrated in Table 4.9, a source mode computed close to first resonance subtracting a series of 15 modes to the exact current, accelerates the convergence of the series from very low frequencies to frequencies close to the third resonance.

With the purpose of verifying that the above described process can be systematically applied to fasten the convergence of any planar antenna problem, next section deals with the approximation of the input current for a coaxial fed patch antenna placed over an infinite ground plane. 


\subsection{Convergence of the series of characteristic modes for coaxial fed patches.}

Figure 4.22 shows the geometry of a rectangular patch whose current is going to be studied. This patch is connected to an infinite ground plane through a narrow vertical strip. The vertical strip emulates a coaxial feeding probe.

Following the approach described in previous section, a source mode is going to be obtained close to the first resonance. The first resonance of this rectangular patch depends on its height over the ground plane. Nevertheless, for this analysis in which the dielectric is air, it will be supposed that the first resonance is close to $3.75 \mathrm{GHz}$, since at this frequency the length of the patch $L$ is $\lambda / 2$.

Then, the total current on the patch is computed at $3.75 \mathrm{GHz}$ by direct inversion of the impedance matrix, and it is also approximated with an expansion of 15 characteristic modes. Figure 4.23 compares the real and imaginary parts of the exact current and the approximated current at $3.75 \mathrm{GHz}$. As observed, both results are very similar. The relative quadratic error at the feeding edge is very small for the real part. However, once more, the $21.7 \%$ error obtained for the imaginary part is too high.

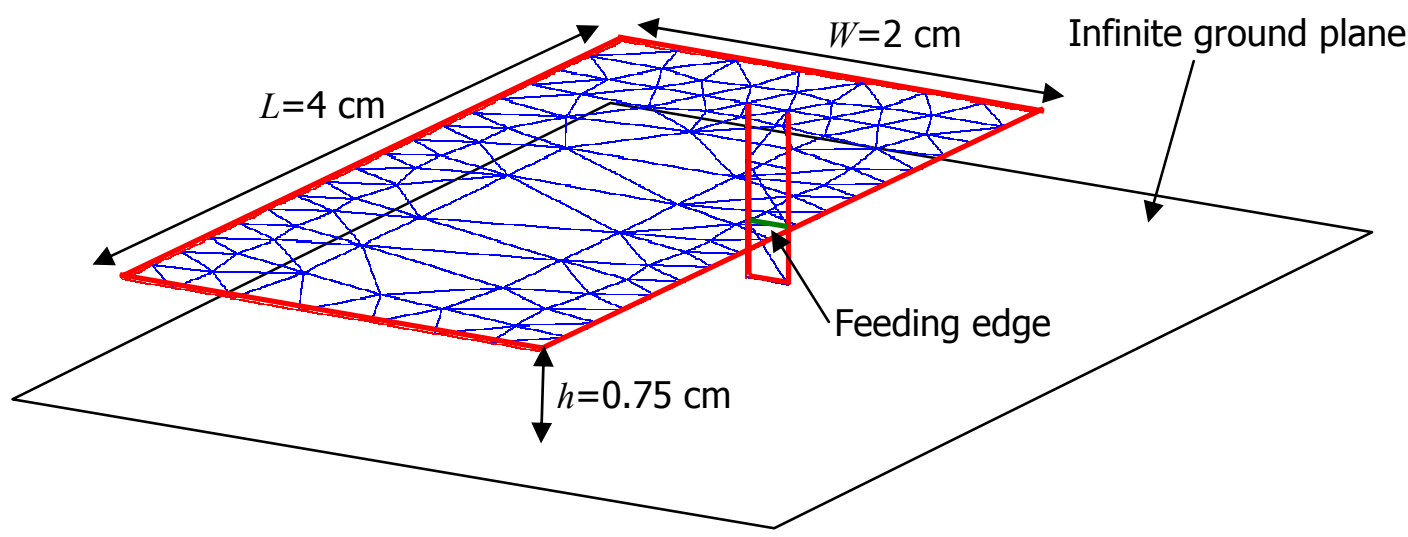

Figure 4.22 Rectangular patch antenna placed over an infinite ground plane.

Next, a source mode is obtained by subtracting to the actual total current at $3.75 \mathrm{GHz}$, the approximation provided by the expansion of 15 characteristic modes. Figure 4.24 shows the current distribution of the residual source mode obtained this way. As observed, the current is very intense at the vertical strip. Due to the complexity of this structure, a linear representation of the current amplitude of the error is not presented, as it does not provide any relevant information. 
In order to perform a SVD decomposition $M=5$ modes are computed at $N=6$ uniformly spaced frequencies between $2.75 \mathrm{GHz}$ and $4.25 \mathrm{GHz}$. The resultant 30 modes plus the source mode shown in Figure 4.24 are arranged as columns vectors to form a new matrix $W$ of dimension $232 \times 31$. Finally, the SVD decomposition is applied to matrix $W$ for obtaining the new set of $P=15$ FICM. This new modes are column vectors of dimension $232 \times 1$.

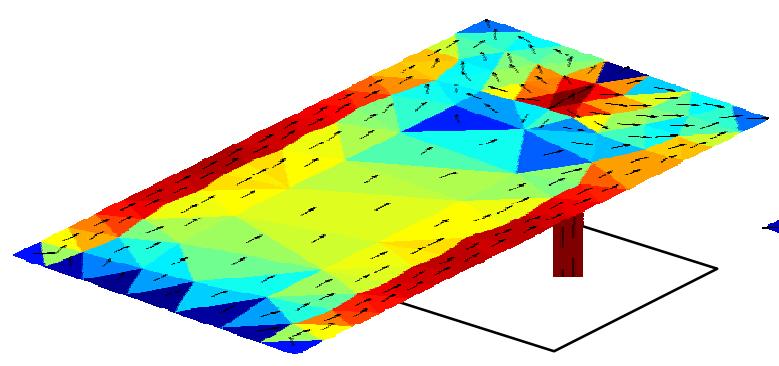

(a) Real part

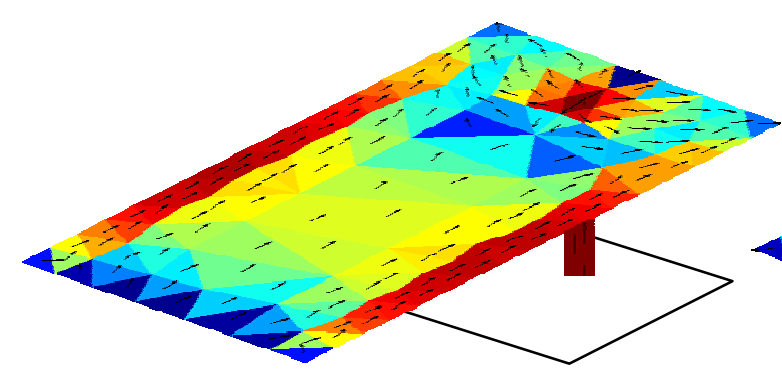

(c) Real part approximated with 15 modes

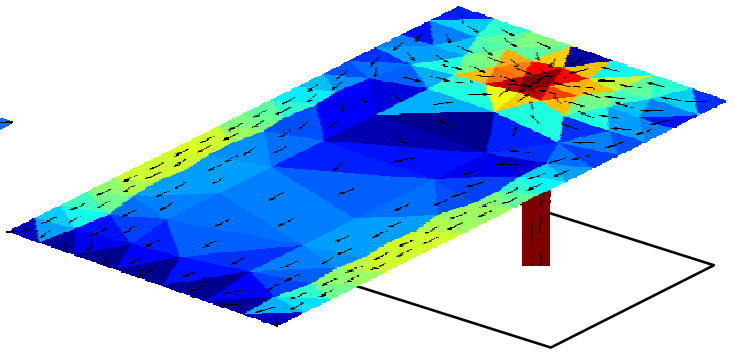

(b) Imaginary part

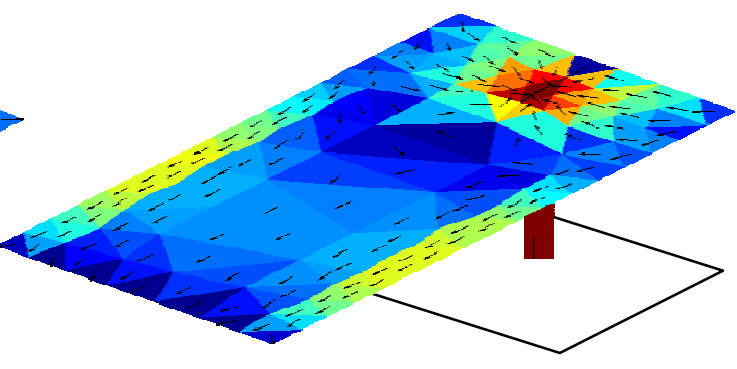

(d) Imaginary part approximated with 15 modes

Figure 4.23 Exact current and current approximated with an expansion of 15 modes at $3.75 \mathrm{GHz}$.

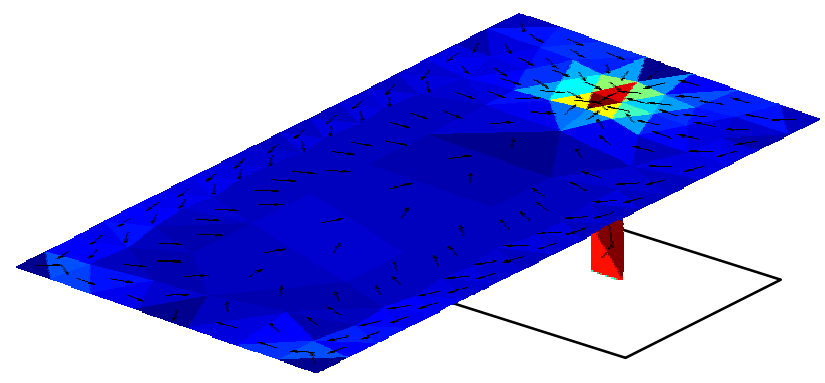

Figure 4.24 Residue that is left after subtracting to the actual total current at $3.75 \mathrm{GHz}$, the approximation yield by an expansion of 15 characteristic modes. 
Table 4.11 presents the relative quadratic error at different frequencies for the input current of the rectangular patch, when it is approximated with the 15 FICM yielded by the SVD decomposition. These results reveal that the convergence of the input current in this example is excellent, since the relative error for the imaginary part stays below $0.1 \%$ at all the studied frequencies.

To sum up, characteristic modes can be used as entire domain basis functions in any planar antenna problem. The slow convergence of the series of modes can be overcome by adding to the series of modes an evanescent mode that models the reactive behaviour introduced by the source. This source mode can be systematically obtained at a frequency close to the first resonance, by subtracting to the exact current an expansion of modes. As it has been demonstrated in section 4.6, a source mode computed this way, is also valid to fasten the convergence at other frequencies.

Finally, if the antenna is to be analyzed in a wide range of frequencies, a SVD decomposition is recommended in order to condense the collection of frequency dependent characteristic modes into a more reduced set of FICM. Examples previously presented demonstrated that with just 15 FICM any planar antenna can be characterized in a wide range of frequencies.

\begin{tabular}{|c|c|c|}
\cline { 2 - 3 } \multicolumn{1}{c|}{} & \multicolumn{2}{c|}{ Expansion with 15 FICM } \\
\hline Frequencies & $\begin{array}{c}\text { \% Relative Quadratic Error } \\
\text { Real part }\end{array}$ & $\begin{array}{c}\text { \% Relative Quadratic Error } \\
\text { Imaginary part }\end{array}$ \\
\hline $1.25 \mathrm{GHz}$ & 0.0794 & 0.02889 \\
\hline $2.5 \mathrm{GHz}$ & 0.0016 & 0.06124 \\
\hline $3 \mathrm{GHz}$ & $0.28 \mathrm{e}-5$ & 0.43637 \\
\hline $3.5 \mathrm{GHz}$ & $7.79 \mathrm{e}-5$ & 0.03835 \\
\hline $3.75 \mathrm{GHz}$ & 0.0004 & 0.02395 \\
\hline $4 \mathrm{GHz}$ & 0.0003 & 0.01752 \\
\hline $4.5 \mathrm{GHz}$ & 0.0062 & 0.01576 \\
\hline $4.75 \mathrm{GHz}$ & 0.012465 & 0.01366 \\
\hline
\end{tabular}

Table 4.11. Relative quadratic error computed at different frequencies when the input current of the rectangular patch is approximated by an expansion of FICM. 


\subsection{Introduction}

Planar antennas, like patches [99]-[101], and planar monopoles [102]-[103], have recently received much attention for wireless communication systems, due to its attractive features such as, low profile, light weight, low cost and easy fabrication. This chapter is intended to analyze these two types of planar antennas using characteristic modes. By means of different examples, it will be demonstrated that from the knowledge gained by the modal analysis, design of new antennas can be performed in a systematic way. In these examples, the information provided by characteristic modes will be used for the selection of the most suitable shape for the radiating element, in some instances, and in others, for the choice of an optimum feeding arrangement.

\subsection{Modal analysis of patch antennas.}

Conventional microstrip antennas are composed of a conducting patch printed on a grounded microwave substrate. Because of their low profile, lightweight, low cost, and conformability, these antennas have long been used for aerospace and mobile applications.

Among the different methods of analysis that have been developed for the study of microstrip patches, the transmission-line model, the cavity model and full-wave models, are the most common ones [86]. The problem with these methods is that all of them present a trade-off between simplicity and accuracy. For example, the transmission-line and the cavity model, are quite easy to implement, however, they do not provide very accurate results and do not model coupling properly. Moreover, although these two methods yield some physical insight, they lack versatility, since they can only be applied to rectangular patches, for the case of the transmission-line model, and to canonical structures, for the case of the cavity model. Conversely, full-wave methods which are based on integral equation formulations, provide rigorous and accurate results. Nevertheless, they are the most complex models and they provide few physical insight.

The Theory of Characteristic Modes can be considered a full-wave method, as it requires of the application of the MoM to obtain the generalized impedance matrix of the antenna. However, as explained in Chapter 1, in contrast with other classical full-wave methods, characteristic modes bring clear insight into the radiation mechanism of the patch. Furthermore, characteristic modes present no limitation over the shape of the patch, the thickness, or the dielectric constant of the substrate. 


\subsubsection{Computation of characteristic modes for arbitrary shaped plates.}

For the present, a rectangular plate of width $W=4 \mathrm{~cm}$ and length $L=6 \mathrm{~cm}$ has been chosen as example to illustrate the behaviour of characteristic modes in planar conducting structures. In first instance, no ground plane has been considered, so the plate is in free space.

Figure 5.1 depicts the current distribution at first resonance $(f=2.2 \mathrm{GHz})$ of the first eigenvectors $J_{n}$ of the rectangular plate already described. Computation of these eigenvectors has been made using 128 RWG functions for expansion and testing. All currents have been normalized to its maximum value in order to facilitate comparison. Additionally, for a better understanding, Figure 5.2 yields current schematics for these eight current modes. Eigenvectors $J_{01}, J_{02}$, and $J_{03}$, as it will be verified later, present special inductive nature due to its currents forming closed loops over the plate. For the case of eigenvector $J_{01}$, the current flows uninterrupted around the plate. Eigenvector $J_{02}$ exhibits currents forming two closed loop in the vertical direction, while currents in eigenvector $J_{03}$ form two closed loops in the horizontal direction. Eigenvectors $J_{1}$ and $J_{2}$, which are characterized by horizontal and vertical currents respectively, are the most frequently used modes in patch antenna applications, while the rest of eigenvectors, $J_{3}, J_{4}$ and $J_{5}$, are higher order modes that might be taken into consideration only at highest frequencies.

Note that due to eigenvectors dependency upon frequency, if a structure is to be analyzed in a wide frequency range, modes will need to be recalculated at every frequency.

Figure 5.3 depicts the azimuthal radiation pattern $\left(\theta=90^{\circ}\right)$ at $4 \mathrm{GHz}$ of the modal electric fields $E_{\theta, n}$ produced by the current modes $J_{n}$ of the rectangular plate. It can be observed that the radiation pattern generated by mode $J_{01}$ presents nearly omni directional characteristic, while the rest of modes present growing number of lobes as the order of the mode increases.

Figure 5.4 presents the variation with frequency of the characteristic angle $\alpha_{n}$ associated to the current modes of the rectangular plate of Figure 5.1. Observe that modes are at resonance when $\lambda_{n}=0$, that is, when its characteristic angle is $\alpha_{n}=180^{\circ}$. Hence, mode $J_{1}$ resonates at $2.3 \mathrm{GHz}$, mode $J_{2}$ at $4.9 \mathrm{GHz}$, mode $J_{3}$ at $4.1 \mathrm{GHz}$, mode $J_{4}$ at $5.6 \mathrm{GHz}$ and mode $J_{5}$ at $9.9 \mathrm{GHz}$. The special nature of the non-resonant inductive modes $J_{01}, J_{02}$, and $J_{03}$, can be appreciated in Figure 5.4, since its associated angle remains below $180^{\circ}$ at every frequency. All modes exhibit broad radiating bandwidth, because their characteristic angle curves are close to $180^{\circ}$ in a wide frequency range after resonance. 

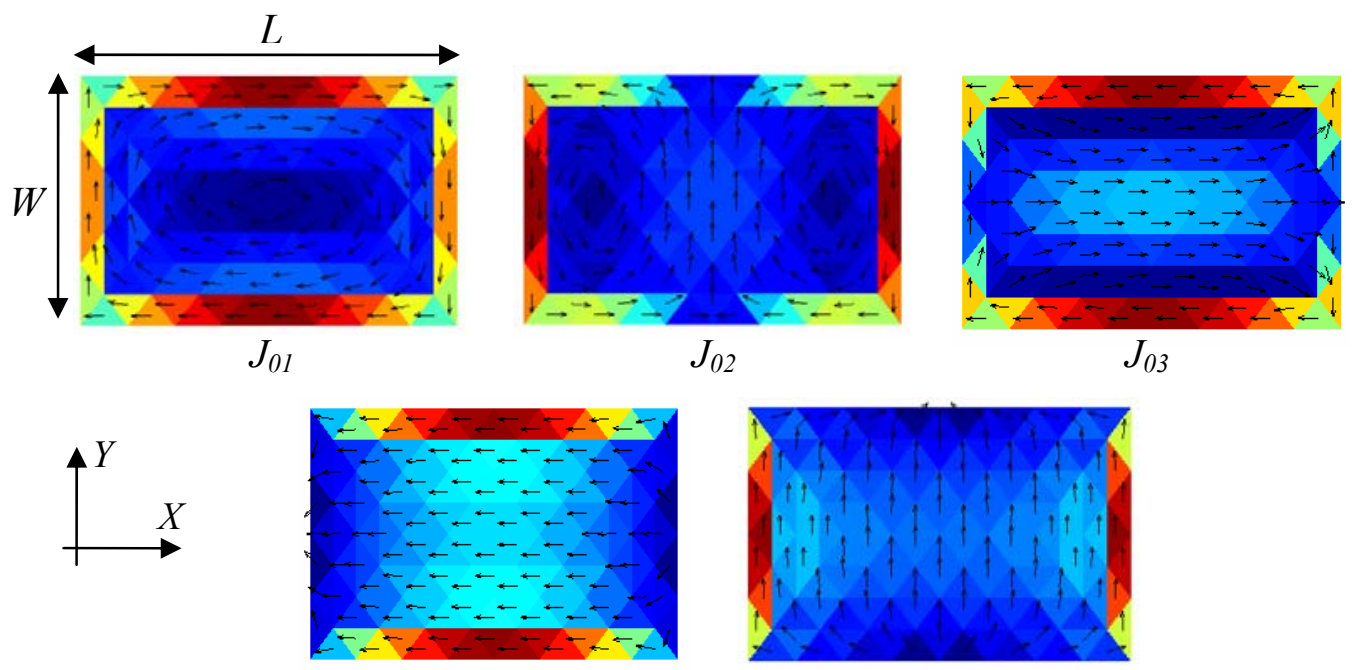

$J_{1}$

$J_{2}$
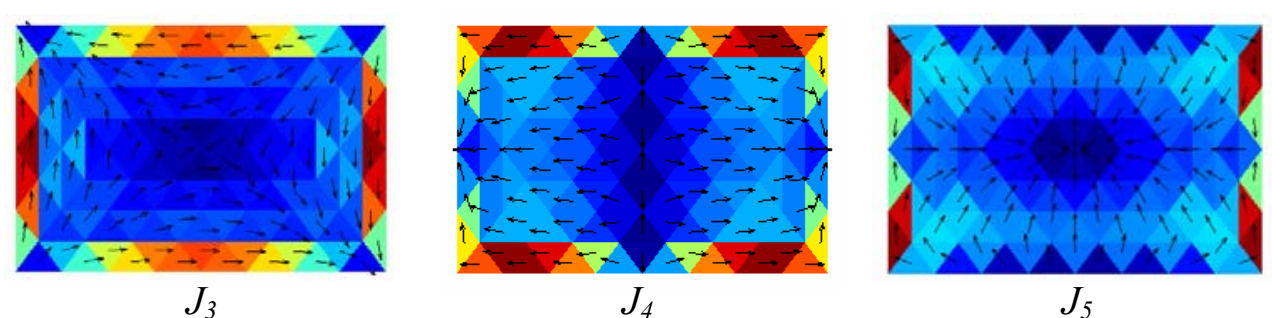

$J_{5}$

Figure 5.1 Normalized current distribution at first resonance $(f=2.3 \mathrm{GHz})$ of the eigenvectors $J_{n}$ of a rectangular plate of width $W=4 \mathrm{~cm}$ and length $L=6 \mathrm{~cm}$.
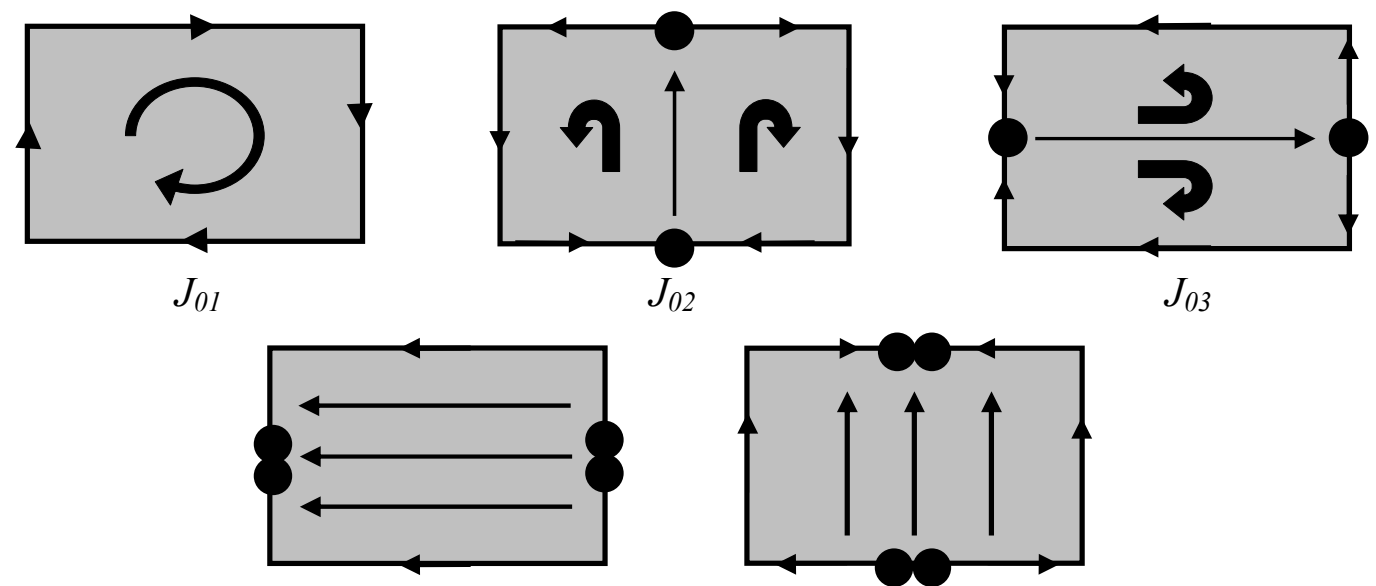

$J_{I}$
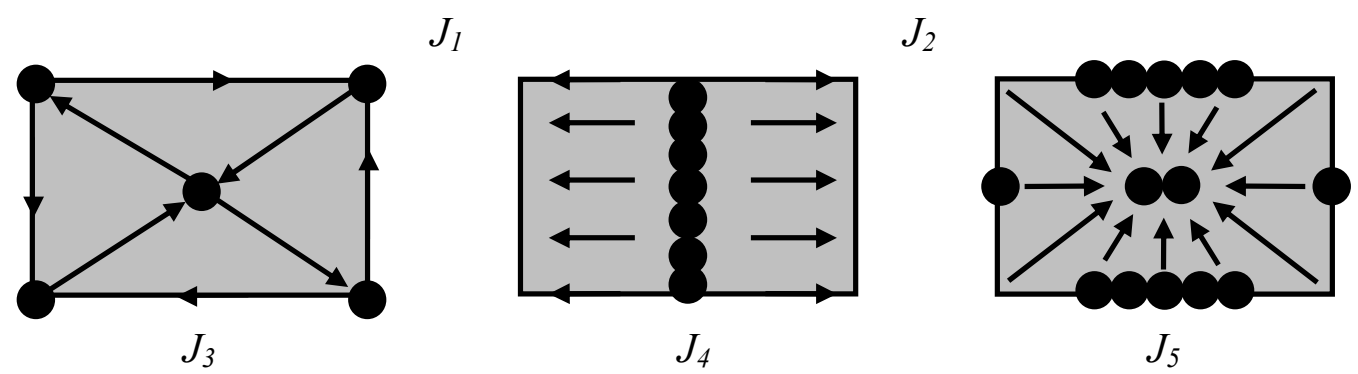

Figure 5.2 Current schematics of the six modes shown in Figure 5.1. 


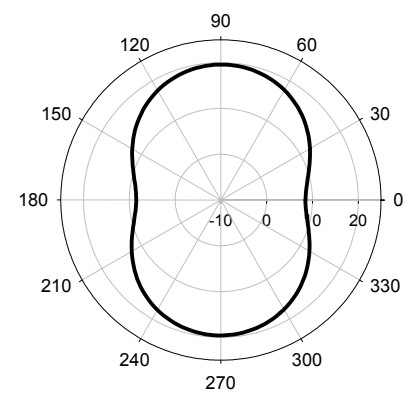

$E_{\theta, 01}$

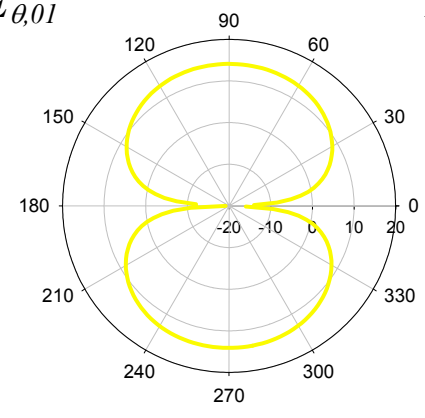

$E_{\theta, 1}$

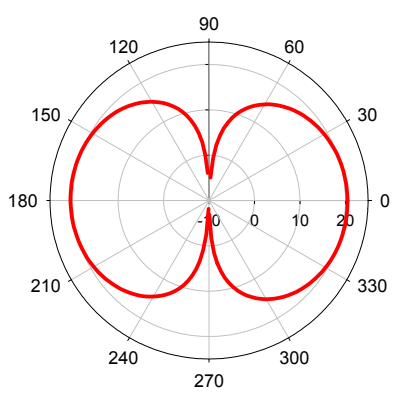

$E_{\theta, 02}$

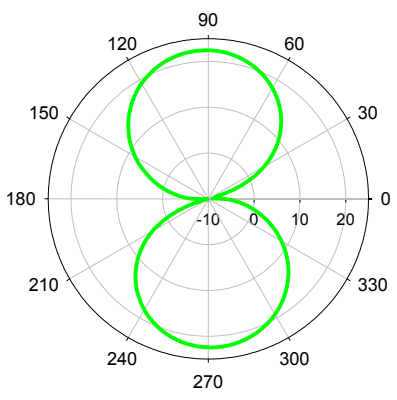

$E_{\theta, 03}$
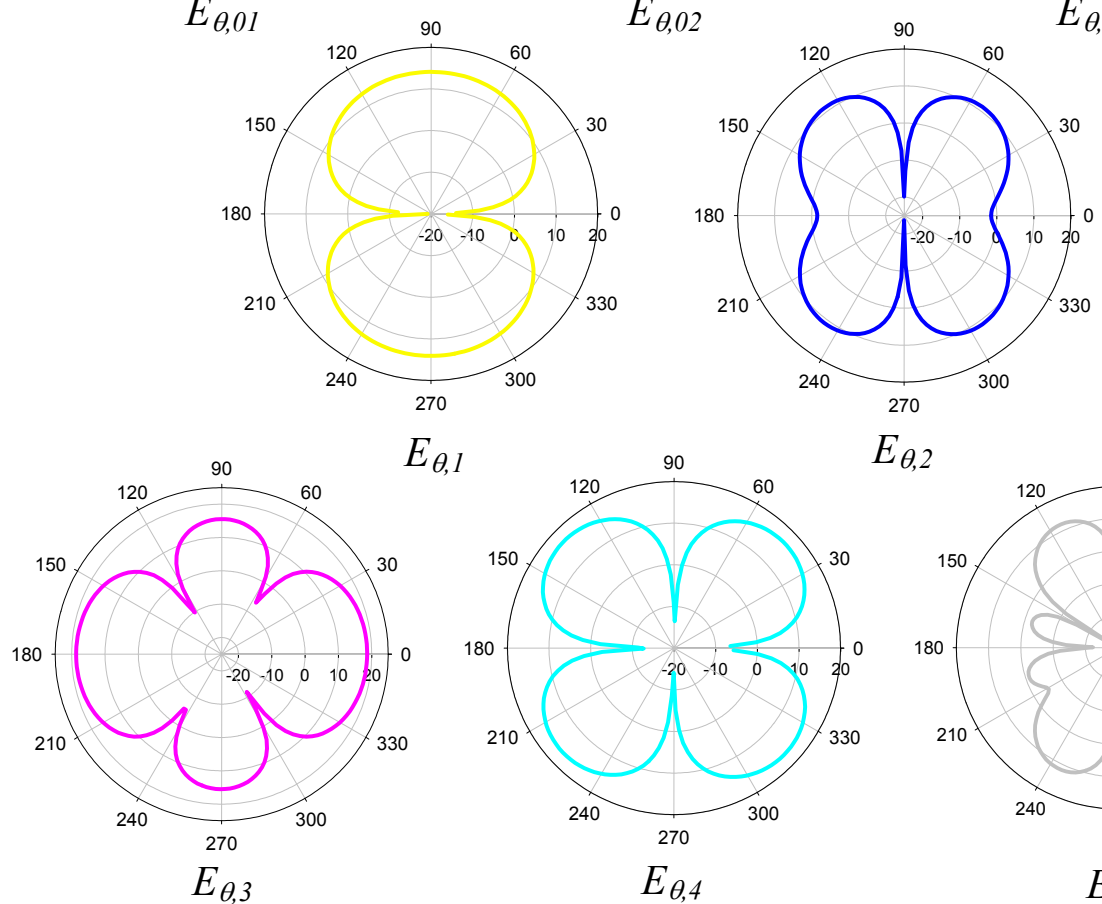

$E_{\theta, 2}$

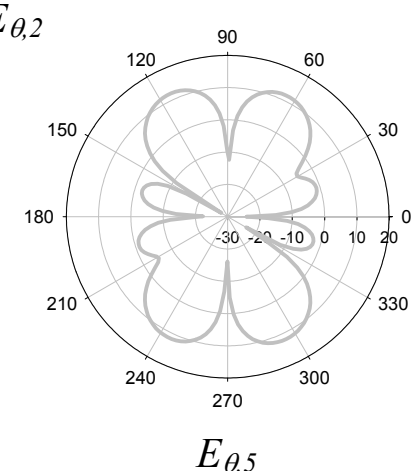

Figure 5.3 Azimuthal radiation pattern $\left(\theta=90^{\circ}\right)$ at $4 \mathrm{GHz}$ of the modal electric fields $E_{\theta, n}$ produced by the current modes $J_{n}$.

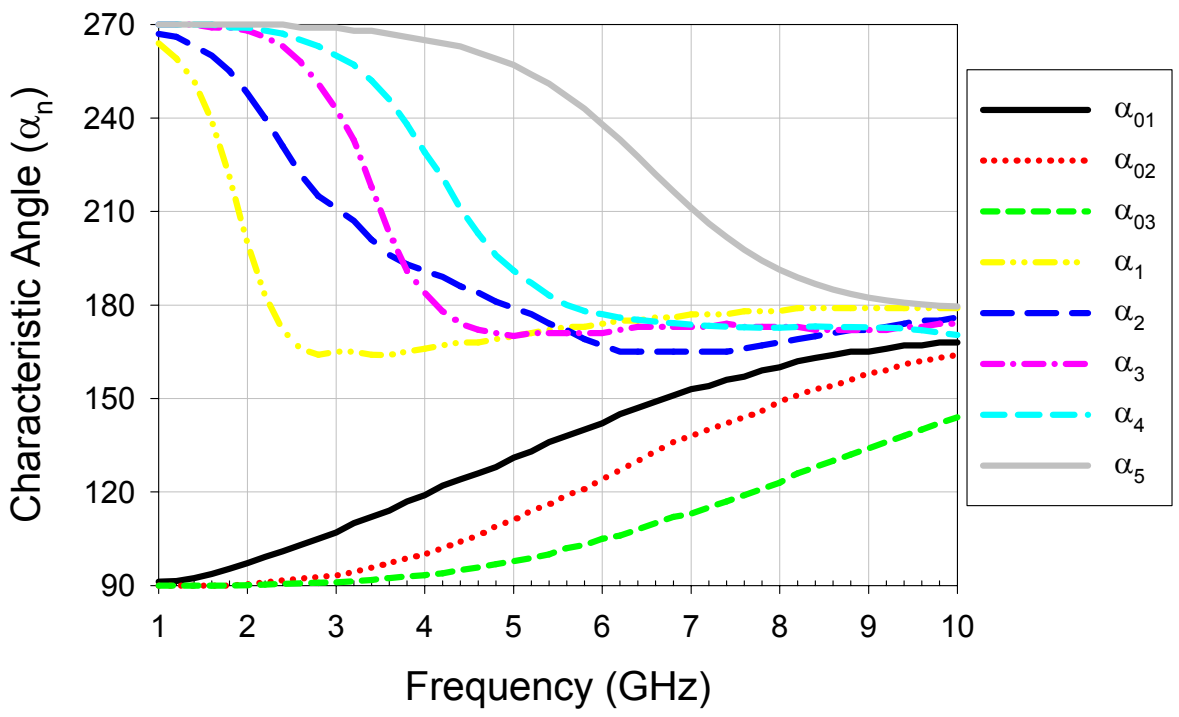

Figure 5.4 Characteristic angle variation with frequency for the current modes of the rectangular plate in free space. 
With the aim of establishing the radiating behaviour of modes in a more precise way, equation (2.24) proposed in chapter two, may be used to compute the modal radiating bandwidth for half-power radiated at resonance. The problem is that this equation is only valid for narrowband radiators. Note frequencies $f_{U}$ and $f_{L}$ in equation (2.24) are the frequencies at which characteristic angles are $135^{\circ}$ and 2250, respectively. In Figure 5.4 it can be observed that after resonance, the characteristic angles of the radiating modes of the rectangular plate stay close to $180^{\circ}$, so they do not go through $135^{\circ}$. In consequence, equation (2.24) can not be applied for obtaining the radiating bandwidth of broadband modes, since only the lower end of the radiating band can be determined from characteristic angle curves. So, according to the information yield by characteristic angle curves, the radiating bandwidth of the modes of the rectangular plate would be infinite.

Another approach to determine the radiating behaviour of modes consists in studying the steepness of the characteristic value curves at resonance [38]. Figure 5.5 illustrates the variation with frequency for the characteristic values of the first modes of the rectangular plate. At first sight, all modes seem to present similar radiating behaviour. Being more concise, a modal quality factor can be calculated from the information provided by Figure 5.5 using equation (2.29). Table 5.1 compares the magnitude of the modal quality factors obtained using (2.26) and (2.29). The radiating bandwidths have been obtained by inverting the corresponding quality factors. As observed, all quality factors are very small. It is a well-known that the inverse dependence between the fractional bandwidth and the quality factor only exists provided $Q>>1$ [49]. Moreover, these quality factors obtained at resonance may not be representatives of the overall radiating behaviour of the modes. Observe that in Figure 5.5, after resonance, the slope of the eigenvalue curves changes into a very flat profile that would result in a zero derivative, and once again, in an infinite radiating bandwidth for low- $Q$ broadband modes after resonance.

Anyway, results presented in Table 5.1, may not be very realistic if it is taken into account that the concept of the quality factor was originated in the context of narrowband resonant circuits, and so, it can not be generalized to any kind of resonators, and it is not particularly suitable for broadband ones.

There are actually other more practical ways to define the bandwidth of an antenna, which include impedance, VSWR, pattern, and gain [104]. All these definitions of the bandwidth can be extrapolated to modes, although they are strongly dependent on the feeding arrangement.

In general, the impedance bandwidth of an excited mode will be not as big as its radiating bandwidth, since it depends on the proper choice of the feeding. Obviously, a very efficient radiating mode will be worthless, if it is not well matched to the source. Likewise, a well matched mode with poor radiating behaviour will not contribute very much to the overall radiation of the antenna. 
Moreover, the interaction of a mode with the rest of modes also degrades its impedance bandwidth. For example, based on the excellent radiating behaviour of mode $J_{l}$ of the rectangular plate, if only this mode were excited, and if it were well matched to the feeding, it would provide a large impedance bandwidth. However, if other modes were excited simultaneously, antiresonances would appear as a result of the combination of modes. Usually, these antiresonances degrade the impedance bandwidth of the antenna, despite the resonances of the interacting modes were closely spaced, and the modes had nearly equal matched resistances, in which case the result would be a wideband performance due to the combination of modes.

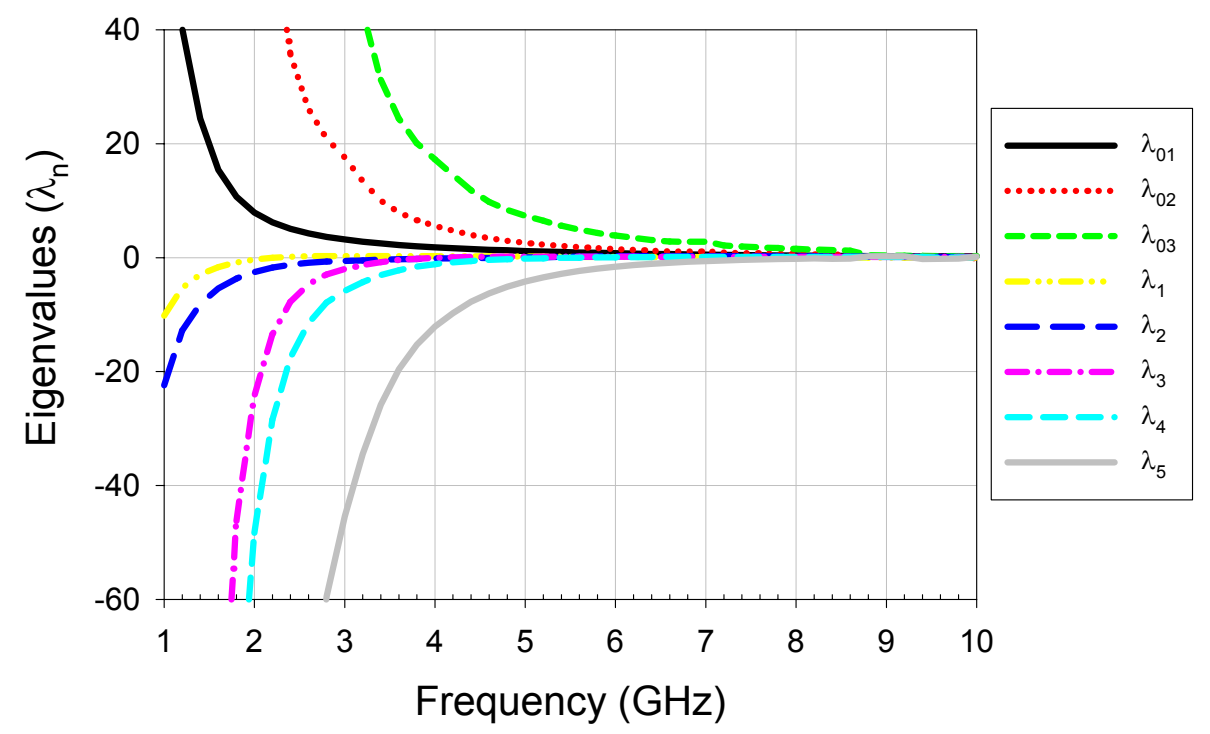

Figure 5.5 Eigenvalue variation with frequency for the current modes of the rectangular plate in free space.

\begin{tabular}{|c|c|c|c|c|}
\cline { 2 - 5 } \multicolumn{1}{c|}{} & $Q_{n, \text { Harrington }}$ & $B W_{n, \text { Harrington }}(\%)$ & $Q_{n, \text { Foster }}$ & $B W_{n, \text { Foster }}(\%)$ \\
\hline Mode $J_{1}$ & 1.09 & $>100 \%$ & 0.814 & $>100 \%$ \\
\hline Mode $J_{2}$ & 0.46 & $>100 \%$ & 0.500 & $>100 \%$ \\
\hline Mode $J_{3}$ & 0.97 & $>100 \%$ & 0.915 & $>100 \%$ \\
\hline Mode $J_{4}$ & 0.59 & $>100 \%$ & 0.490 & $>100 \%$ \\
\hline Mode $J_{5}$ & 0.75 & $>100 \%$ & 0.680 & $>100 \%$ \\
\hline
\end{tabular}

Table 5.1. Modal quality factors computed using (2.29), and modal radiating bandwidths obtained by inversion of the quality factors, for the first five radiating modes of the rectangular plate in free space. 
It is worth noting that eigenvectors presented in Figure 5.1 have been computed in free space, however, the presence of a ground plane below the plate would not alter significantly their current distribution, although it would affect their resonance and radiating bandwidth. Figure 5.6 shows the variation with frequency of the characteristic angles associated to the first eight modes of the same rectangular plate, when it is placed $0.8 \mathrm{~mm}$ above an infinite perfect conducting ground plane. It is observed that now modes resonate at a lowest frequency. Mode $J_{1}$ resonates at $2.1 \mathrm{GHz}$, mode $J_{2}$ at $2.9 \mathrm{GHz}$, mode $J_{3}$ at 3.75 $\mathrm{GHz}$, mode $J_{4}$ at $4.35 \mathrm{GHz}$, and mode $J_{5}$ at $6.55 \mathrm{GHz}$. Furthermore, as it was expected, the presence of the ground plane reduces the radiating bandwidth of all modes. In next section, it will be analyzed in more detail how the height of the patch above the ground plane affects the radiating bandwidth of modes.

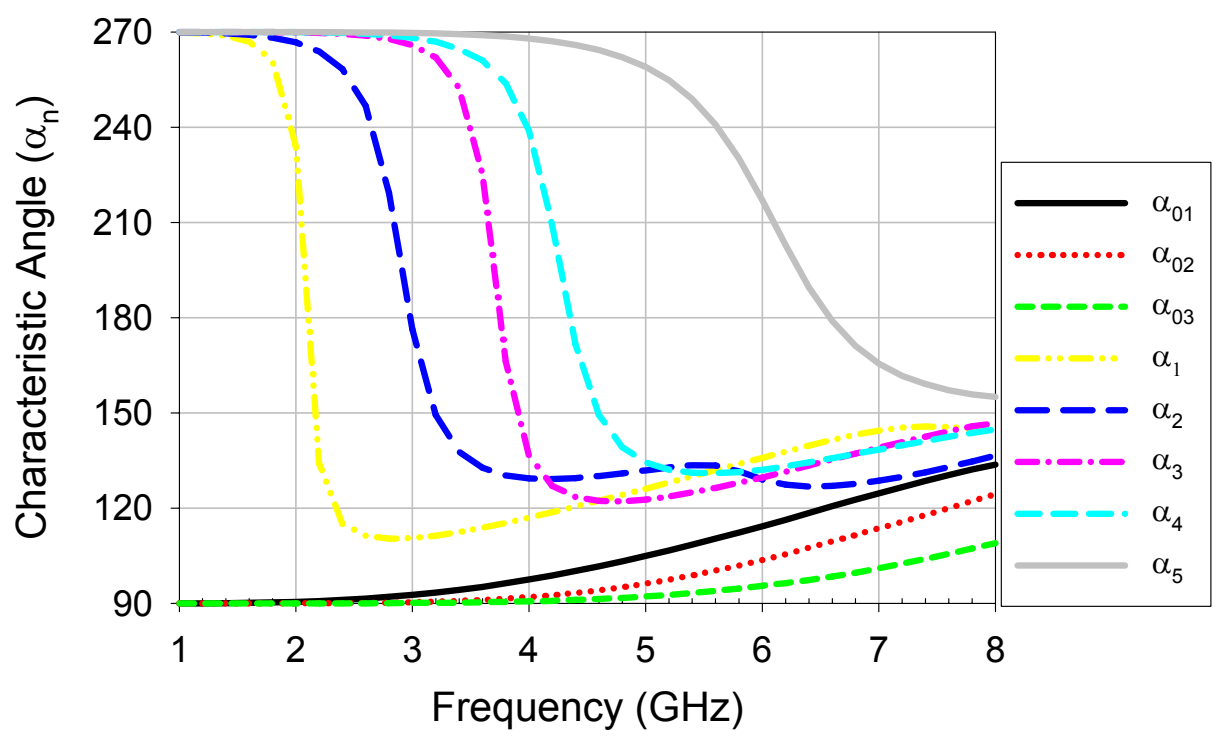

Figure 5.6 Characteristic angle variation with frequency for the current modes of the rectangular plate placed $0.8 \mathrm{~mm}$ above an infinite ground plane.

To sum up on the whole, it should be emphasized that the modal study presented here for the rectangular plate could have also been performed for planar structures of any shape. In fact, characteristic modes present quite a predictable behaviour in planar structures, whichever their shape is. For instance, Figure 5.7 shows the normalized current distribution of different modes at $2.4 \mathrm{GHz}$ for several planar geometries. From these results it can be derived that first mode $J_{I}$ is always characterized by horizontal current flow, except for the contour, where it follows the perimeter of the structures. The second mode $J_{2}$ presents vertical currents along the different plates, exception made of the contour. Likewise, the rest of modes exhibit easily identifiable current patterns. Mode $J_{3}$ has a combination of vertical and horizontal currents, while mode $J_{4}$ is a higher order horizontal mode, and mode $J_{5}$ presents currents flowing in radial direction. 

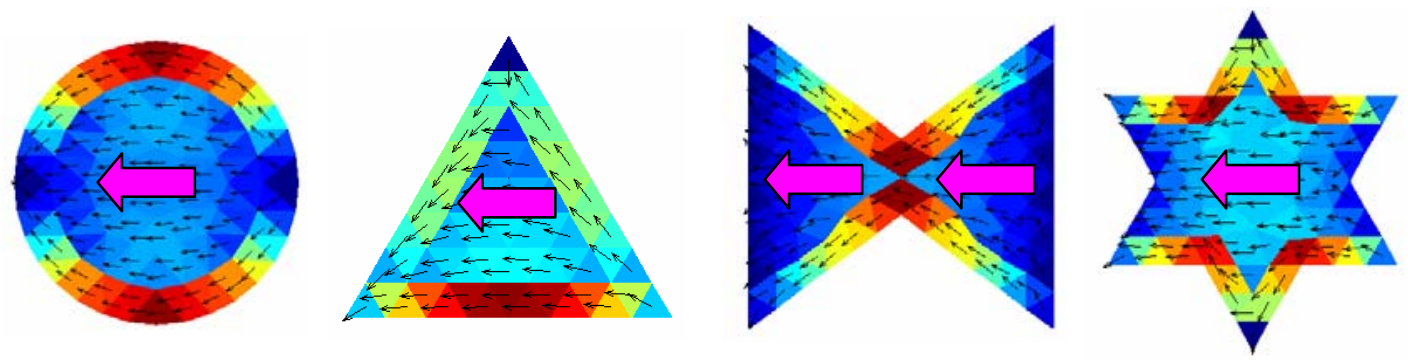

(a) Horizontal current mode $J_{1}$
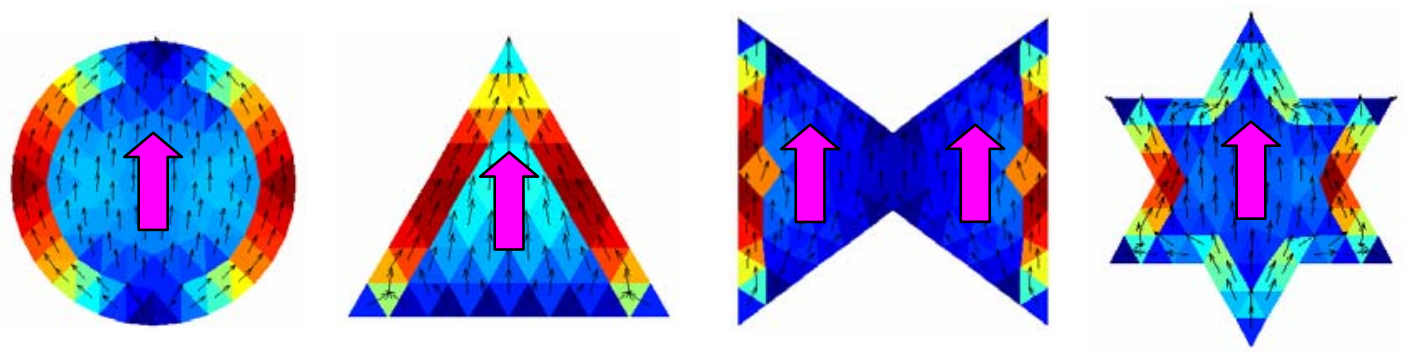

(b) Vertical current mode $J_{2}$
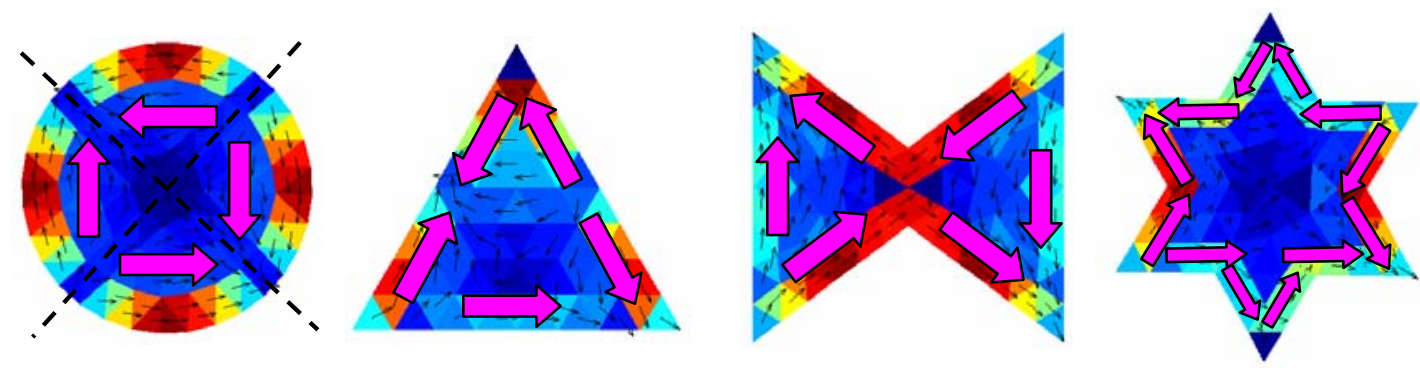

(c) Higher order mode $J_{3}$
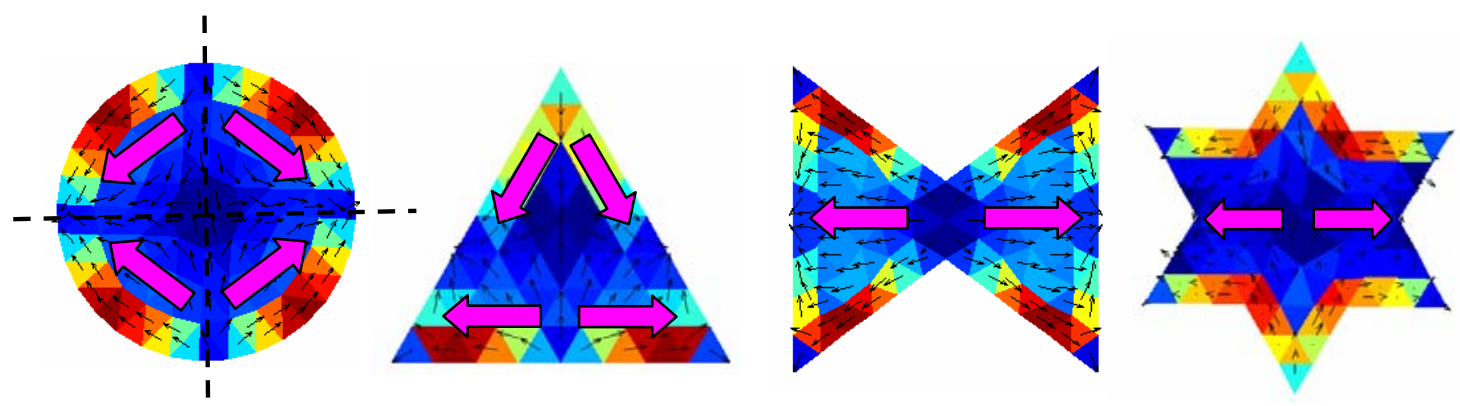

(d) Higher order mode $J_{4}$
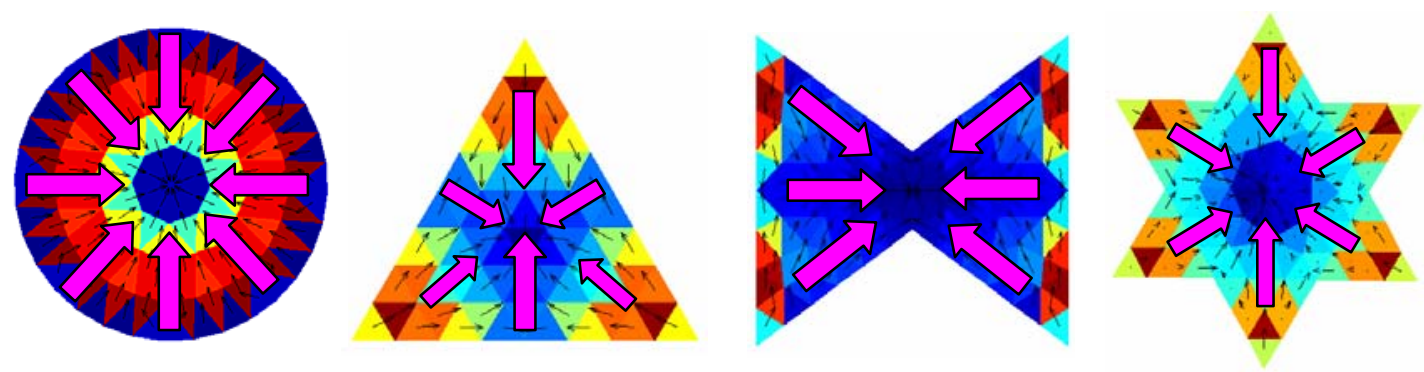

(e) Higher order mode $J_{5}$

Figure 5.7 Normalized current distribution at $2.4 \mathrm{GHz}$ of the first eigenvectors of several planar geometries. 


\subsubsection{Determination of the optimum height of a microstrip patch above a ground plane to obtained maximum radiating bandwidth.}

As it has been mentioned before, when a plate is placed above an infinite ground plane, the current distribution of the eigenvectors remains more or less the same as the one obtained for the free space case. Nevertheless, the presence of a ground plane below the structure has great effect on the resonance frequency and radiating bandwidth of modes. Bandwidth is an important matter of concern for microstrip patches, since they are inherently narrow bandwidth antennas. As a result, bandwidth enhancement techniques are usually needed for most practical applications. Some of these techniques are quite complex, as they consist in mounting stacked elements [105]-[107], adding parasitic elements [108]-[109] or using resistive loading [110]-[111]. However, an easier alternative to increase the bandwidth of a microstrip patch is based on etching the radiating element on a thick low dielectric constant substrate [86].

Next results exemplify how the variation of the height $h$ over an infinite ground plane with air substrate $\left(\varepsilon_{r}=1\right)$, affects the characteristic angle curve of the vertical current mode of the rectangular patch of width $W=4 \mathrm{~cm}$ and length $L=6 \mathrm{~cm}$, depicted in Figure 5.8. The current distribution of this vertical current mode corresponds with the one already plotted for eigenvector $J_{2}$ in Figure 5.1.

Figure 5.9 shows the variation with frequency and height of the characteristic angle associated to this vertical current mode of the rectangular patch. The height $h$ is expressed in terms of the wavelength $\lambda$ at $3.75 \mathrm{GHz}$, since at this frequency, the resonant dimension of the patch is $W=\lambda_{3.75 \mathrm{Gz}} / 2$. The behaviour of the mode in free space is also included for comparison purposes. By observing Figure 5.9 it can be deduced that the maximum radiating bandwidth of the vertical current mode occurs when the height over the ground plane is $h=0.25 \lambda_{3.75 \mathrm{Gr}}$. This conclusion is based on the fact that for $h$ $=0.25 \lambda_{3.75 \mathrm{GH}}$, the characteristic angle curve passes through $180^{\circ}$ three times, approximately at $2.8 \mathrm{GHz}, 4.5 \mathrm{GHz}$ and $5.4 \mathrm{GHz}$, while it keeps close to $180^{\circ}$ at the rest of frequencies. It should be also remarked that because the thickness of the substrate or the dielectric constant, are not constraints for this method, characteristic modes constitute an alternative to analyze patch antennas when the classical model of resonant cavities fails.

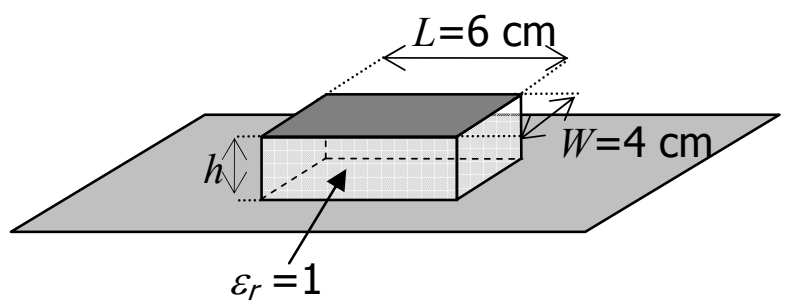

Figure 5.8 Dimensions of the rectangular patch placed over an infinite ground plane with air dielectric. 


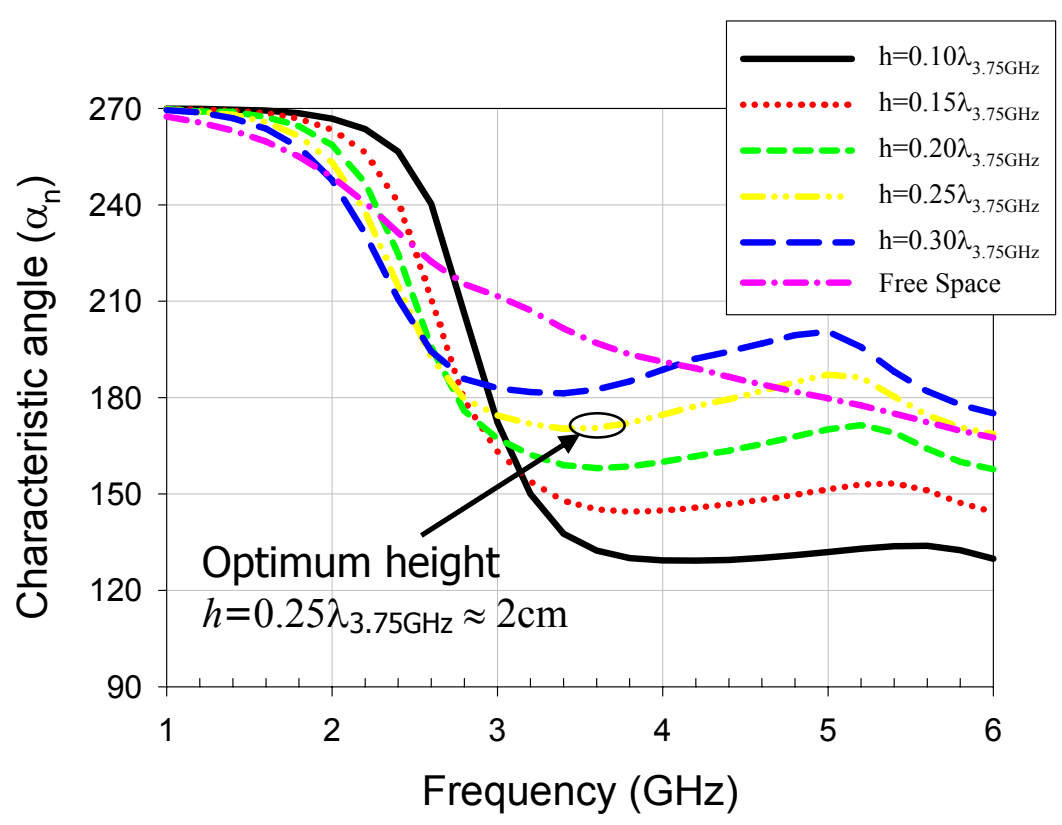

Figure 5.9 Variation with frequency and height $h$ of the characteristic angle associated to the vertical current mode of the rectangular patch.

\subsubsection{Generation of circular polarization in arbitrarily shaped patch antennas by combination of modes.}

It is a well-known fact that, to get circular polarization from a microstrip patch, it is necessary to excite two orthogonal and linearly polarized modes, with equal current amplitude but in phase quadrature [86]. Orthogonality properties of characteristic modes make the generation of circular polarization in arbitrary patches possible in an easy and intuitive way.

For the sake of example, let us describe the procedure carried out to design a circularly polarized isosceles triangular patch antenna using characteristic modes. A triangular patch antenna has already been demonstrated to provide circular polarization [112], so the purpose of this section is not presenting a new antenna, but exemplifying how to design it using modes. The dimensions of the triangular patch are shown in Figure 5.10, and the current distribution at 3.4 $\mathrm{GHz}$ of the modes to be combined, which are the horizontal and the vertical current ones, is shown in Figure 5.11.

From the information provided by the modal significance curves in Figure 5.12 it can be determined that both modes present exactly the same current amplitude at $3.4 \mathrm{GHz}$. Moreover, from the characteristic angle curves presented in Figure 5.13 it is derived that at $3.4 \mathrm{GHz}$ both modes present $90^{\circ}$ phase difference. Hence, if these two modes were properly excited and combined, they would yield circular polarization at $3.4 \mathrm{GHz}$. So, next step is to identify where the feed point should be located to excite these modes 
Figure 5.14 shows the optimum feed position where the two modes present exactly the same current amplitude. This point corresponds with the minimum value obtained after subtracting the two current distributions in Figure 5.11 at 3.4 GHz. Last of all, the axial ratio plotted in Figure 5.15, which has been obtained using an aperture feed with a $45^{\circ}$ rotation, located at the previously specified point, attests that the triangular patch is circularly polarized at broadside direction at $3.4 \mathrm{GHz}$.

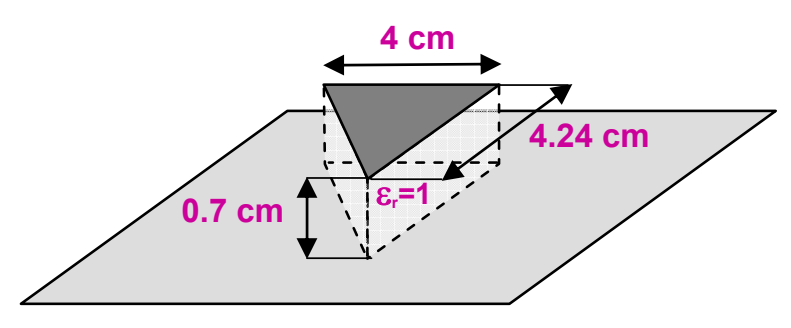

Figure 5.10 Dimensions of the triangular patch antenna placed over an infinite ground plane with air dielectric.
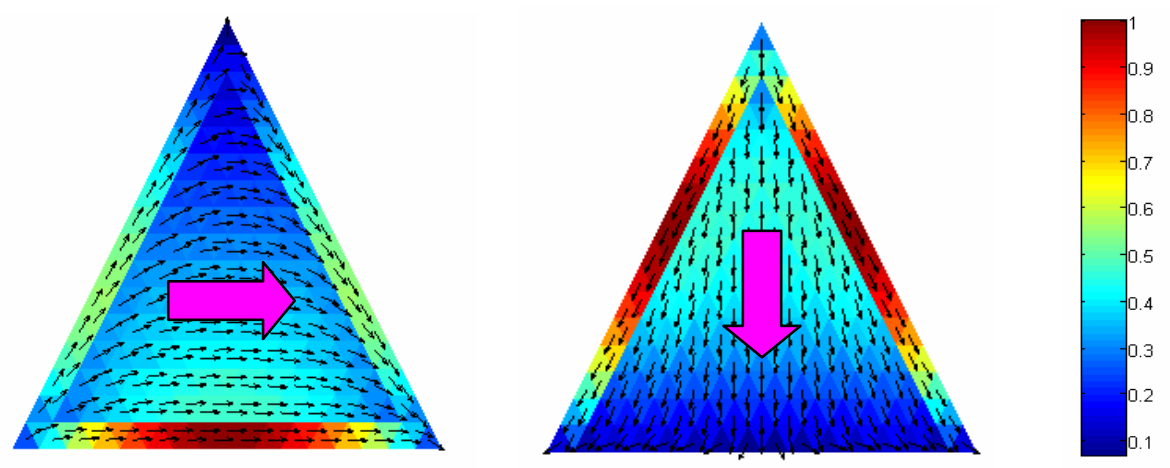

Figure 5.11 Current distribution at $3.4 \mathrm{GHz}$ for the horizontal and vertical current modes of the triangular patch.

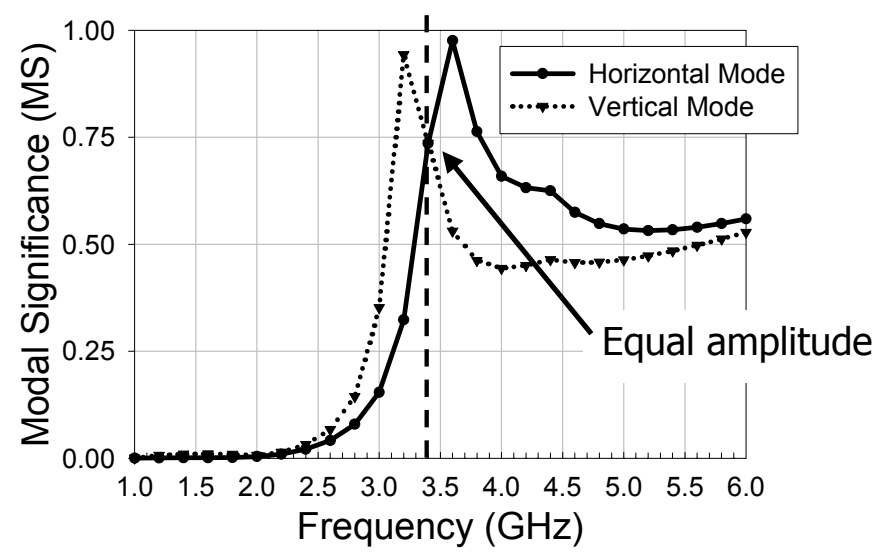

Figure 5.12 Modal significance curves for the horizontal and vertical current modes of a triangular patch. 


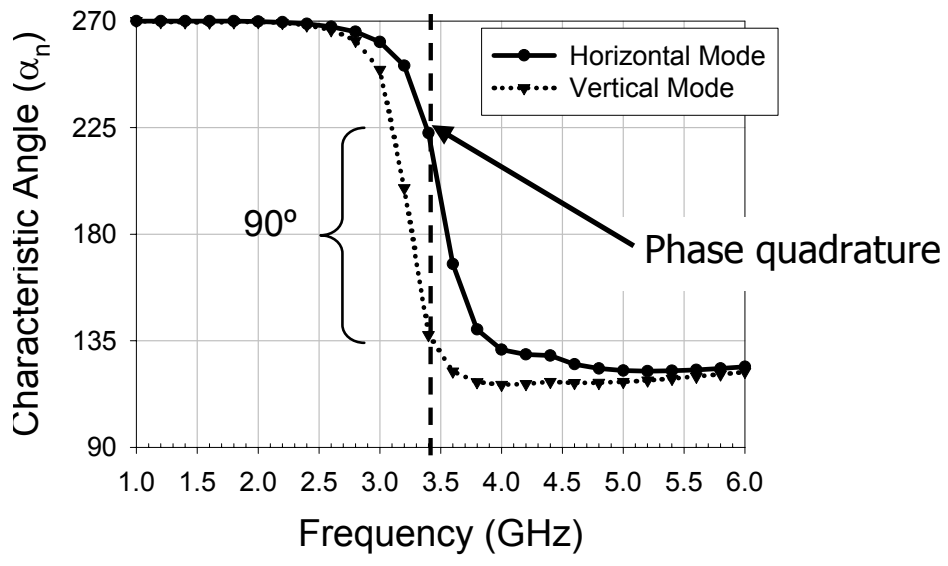

Figure 5.13 Characteristic angle variation with frequency for the horizontal and vertical current modes of a triangular patch.
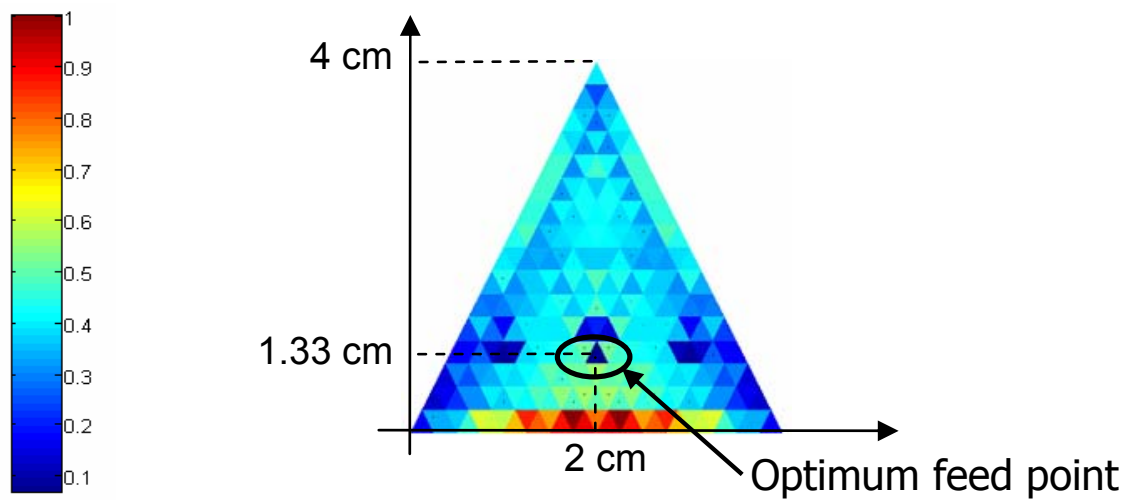

Figure 5.14 Optimum feed position to obtain circular polarization in a triangular patch.

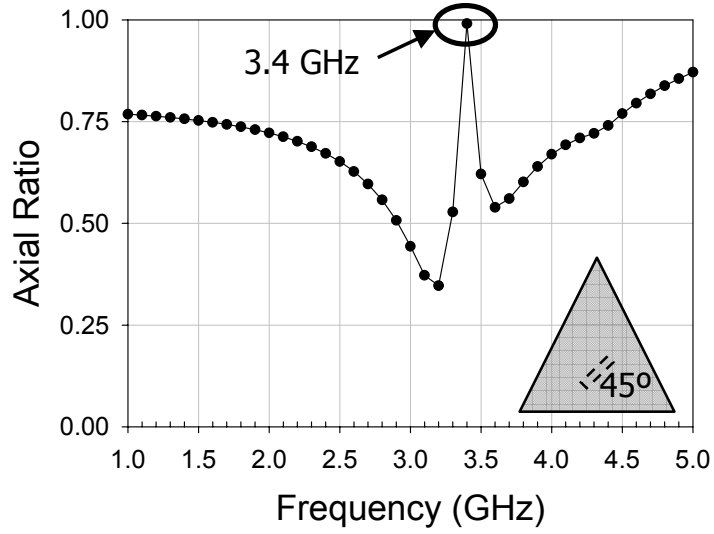

(a)

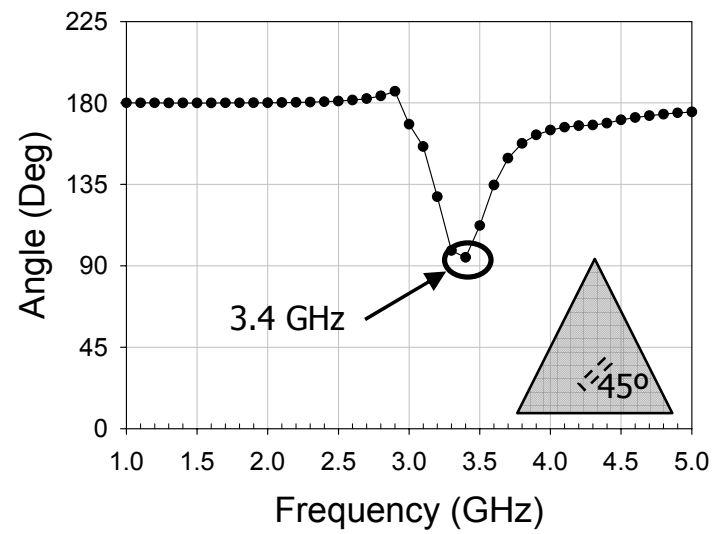

(b)

Figure 5.15 Aperture-coupled feed at 450: (a) Axial ratio at broadside direction, (b) Phase difference at broadside direction. 


\subsubsection{Reduction of the cross polarization level for reflectarray antennas.}

Another interesting application of characteristic modes is to adjust the phase of the field reflected by the individual elements of a reflectarray antenna [113][115]. As described in [30] eigenvalues $\lambda_{n}$ are related to the scattering coefficient $S_{n}$ as

$$
S_{n}=-\frac{1-j \lambda_{n}}{1+j \lambda_{n}}
$$

Then, the reflection phase of the $n^{\text {th }}$ mode can be expressed as

$$
\varphi_{n}=-2 \tan ^{-1}\left(\lambda_{n}\right)
$$

Since equation (5.2) does not depend on the excitation, the total reflection phase, when considering the illuminating feed, will be a combination of the reflection phases of the excited modes

Typically, square and rectangular patches are the most widely used elements for reflectarray applications due to their simplicity, yet they do not always provide the desired bandwidth performance [116]. Recently, the use of ridges has been proposed in order to improve the bandwidth performance of rectangular patches [117], however this solution degrades the cross-polarization level, especially for the case of oblique incidence. In general, a square patch excited by an oblique incident plane wave presents currents on its surface flowing in diagonal direction.

Figure 5.16 (a) shows the current distribution at $8 \mathrm{GHz}$ generated by a $\phi$ polarized incident plane wave in the direction $\theta=30^{\circ}$ and $\phi=45^{\circ}$, over a square patch of dimension $15 \mathrm{~mm}$, placed $3 \mathrm{~mm}$ above an infinite ground plane. This current flows in diagonal direction $\left(-45^{\circ}\right)$, because of the excitation of two orthogonal degenerated modes: The vertical current mode (Figure 5.16 (b)), and the horizontal current mode (Figure 5.16 (c)). The radiation patterns in the $X Z$ and $Y Z$ planes due to the total current are plotted in Figure 5.17. As a result of the excitation of the two degenerated modes, the theta and phi components of the electric field are present in both planes.

One solution to improve the polarization purity of the square patch for the case of oblique incidence is to split the degenerated modes, so only one of them would be excited at the desired frequency. This can be accomplished just by dividing the patch in two rectangular strips along the direction of the desired polarization. 


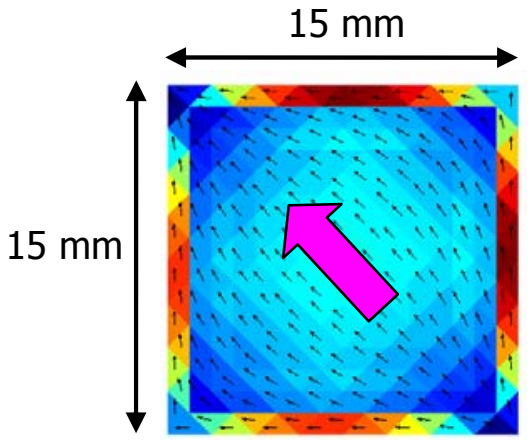

(a)

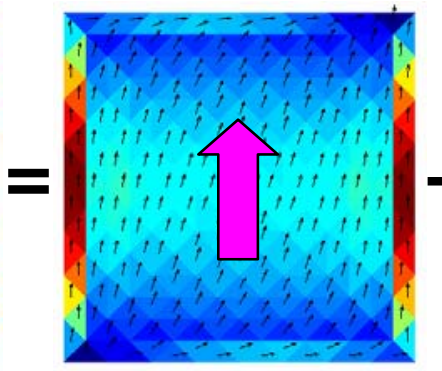

(b)

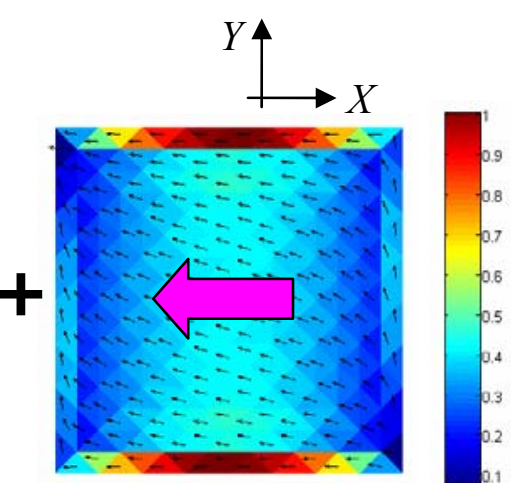

(c)

Figure 5.16 Total current at $8 \mathrm{GHz}$ of a square patch when excited by a $\phi$ polarized incident plane wave $\left(\theta=30^{\circ}, \phi=45^{\circ}\right)$. (b) Vertical current mode at 8 $\mathrm{GHz}$. (c) Horizontal current mode at $8 \mathrm{GHz}$.

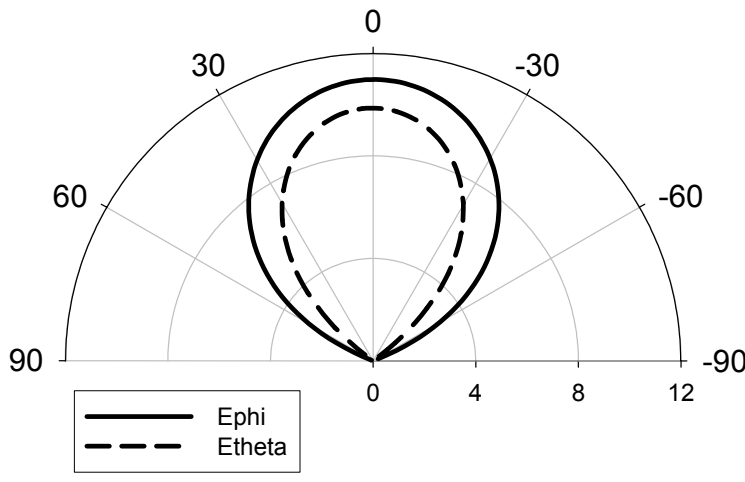

$X Z$ Plane

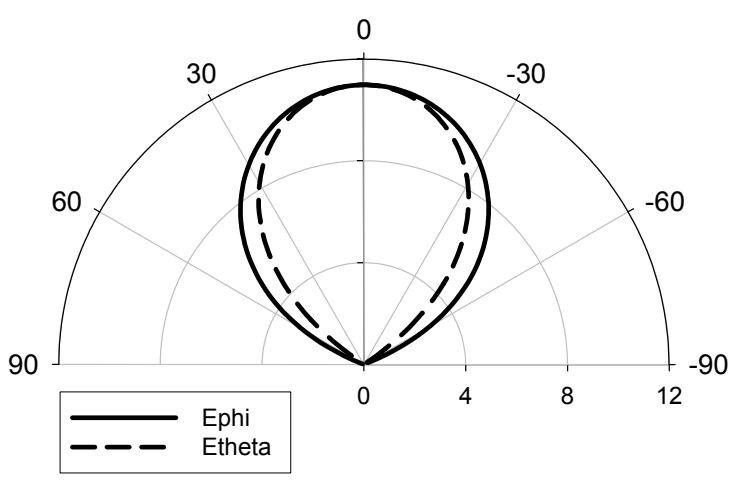

YZ Plane

Figure 5.17 Radiation patterns in the $X Z$ and $Y Z$ planes due to the diagonal current plotted in Figure 5.15 (a).

Figure 5.18 illustrates the normalized current distribution at $8 \mathrm{GHz}$ of the first three modes of the square patch divided in two vertical strips. When the square is divided along the $Y$-axis direction, the vertical mode is preserved, while the horizontal mode, whose current is interrupted by the gap, resonates at a higher frequency. Additionally, a new vertical mode, with currents flowing with opposite phase in the strips, also appears. Figure 5.19 shows the reflection phase versus frequency curves associated to the aforementioned modes of the two vertical strips. This reflection phase has been obtained using equation (5.2). For the sake of comparison, Figure 5.19 also includes the reflection phase of the vertical mode of the square patch. From these results it can be extracted that the vertical current mode of the two vertical strips presents the same reflection phase as the vertical mode of the complete square patch, and also that the resonance $\left(\varphi_{n}=0\right)$ of the horizontal mode is shifted to higher frequencies. 


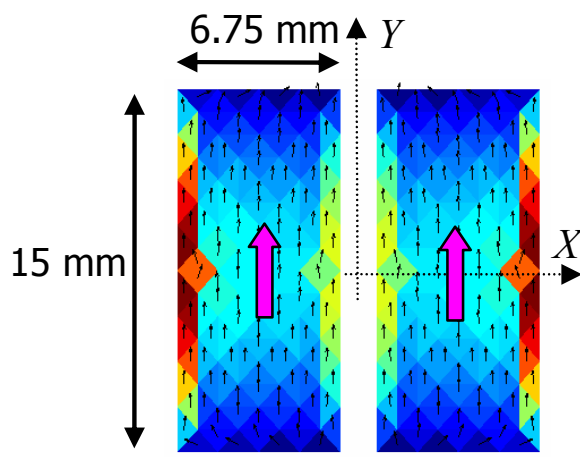

(a)

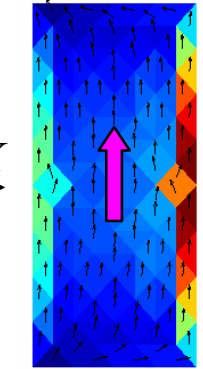

(b)

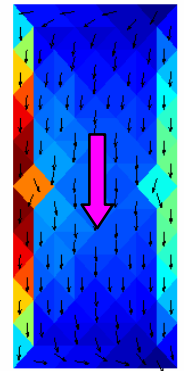

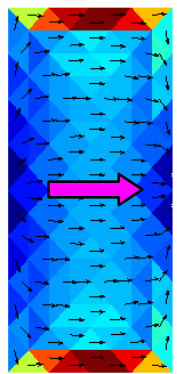

(c)

Figure 5.18 Normalized current distribution at $8 \mathrm{GHz}$ for the first modes of two vertical strips placed $3 \mathrm{~mm}$ above an infinite ground plane. (a) Vertical current mode, (b) Vertical current mode with currents flowing in opposite way, (c) Horizontal current mode.

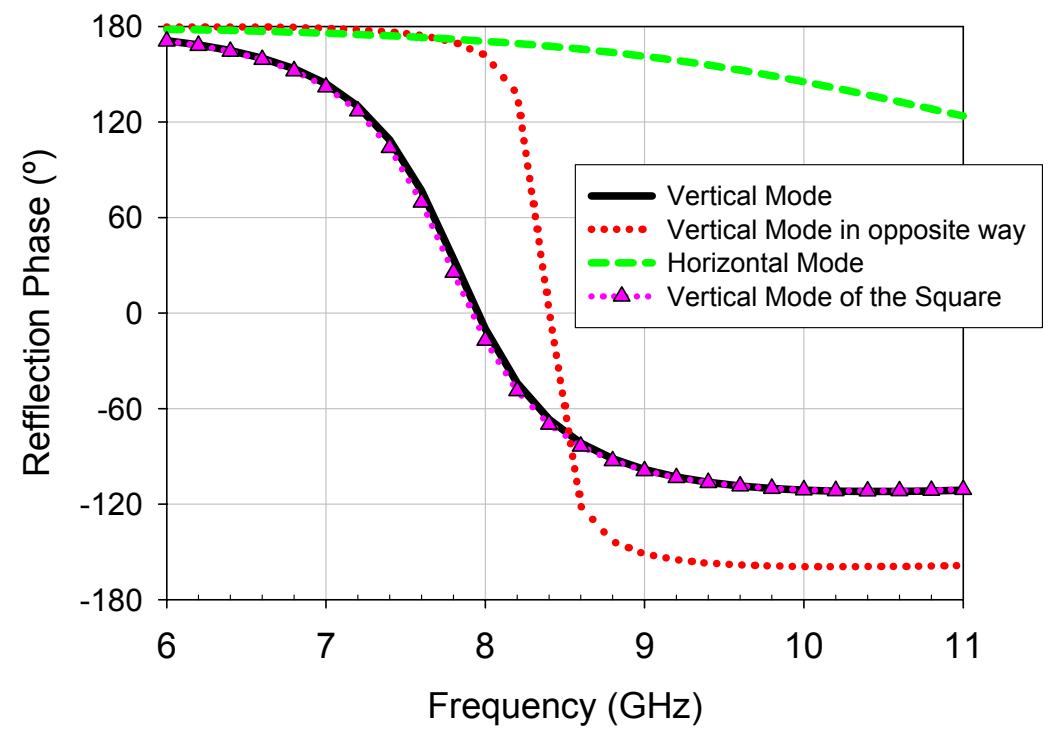

Figure 5.19 Reflection phase against frequency for the modes of the two vertical strips, and for the vertical mode of the square patch.

Finally, when the vertical strips are placed $3 \mathrm{~mm}$ above an infinite ground plane and excited with the $\phi$-polarized incident plane wave used in the previous case, it results in the current sketched in Figure 5.20. This current that flows in vertical direction, resembles very much the vertical current mode shown in Figure 5.18 (a). This means that only the vertical current mode is excited. Figure 5.21 shows the radiation patterns in the $X Z$ and $Y Z$ planes generated by the current plotted in Figure 5.20. Now, it is observed that the phi component of the electric field is only present at the $X Z$ plane, and the theta component is only present at the $Y Z$ plane. 


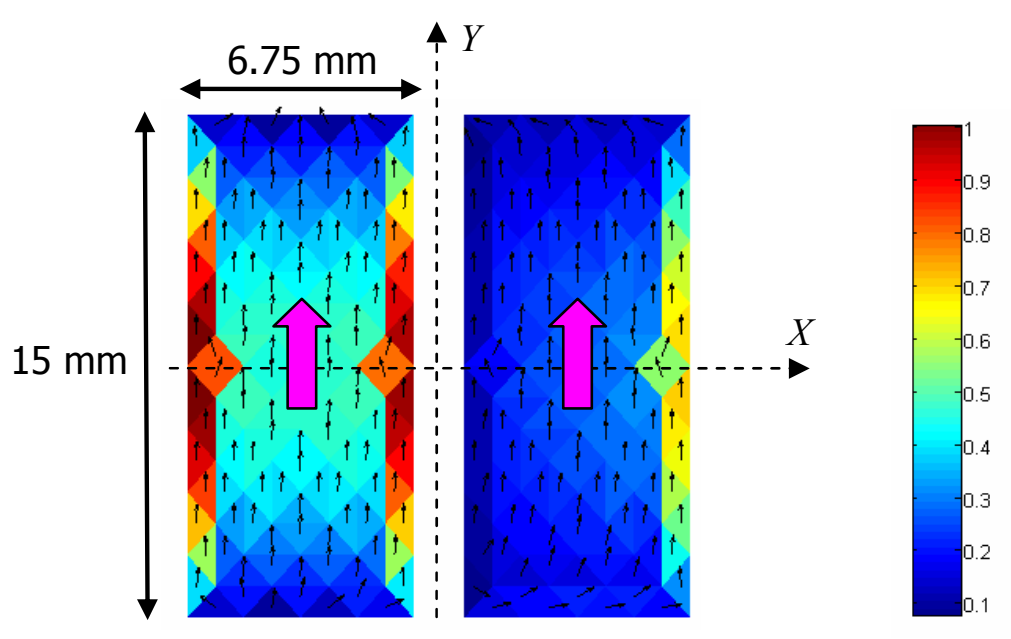

Figure 5.20 Total current at $8 \mathrm{GHz}$ of two vertical strips when excited by a $\phi$ polarized incident plane wave in the direction $\theta=30^{\circ}, \phi=45^{\circ}$.

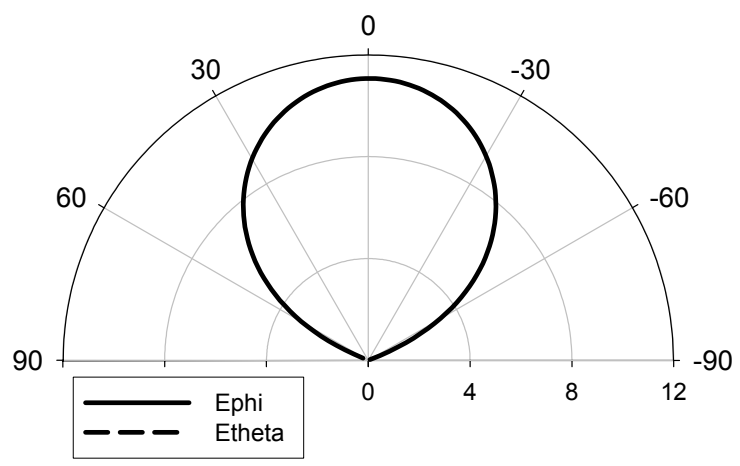

$X Z$ Plane

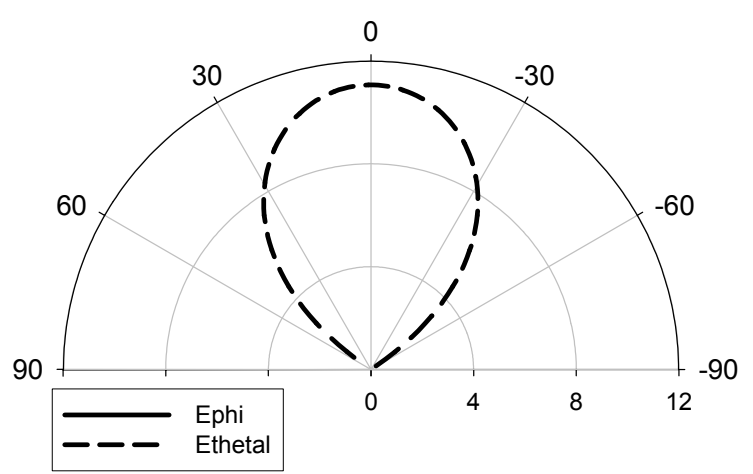

YZ Plane

Figure 5.21 Radiation patterns in the $X Z$ and $Y Z$ planes generated by the current plotted in Figure 5.18.

To sum up, by means of characteristic modes it has been demonstrated that for the case of oblique incidence the polarization purity of a square patch can be improved just by dividing the patch in two rectangular strips in the direction of the desired polarization. With this simple modification of the square patch, the bandwidth performance of the fundamental mode is preserved, while the crosspolar component is very much reduced. 


\subsubsection{Coupling between planar structures.}

From section 5.2.4, it comes up that characteristic modes can not only be obtained for an isolated radiator, but also for an array formed by several of them. In particular, the structure analyzed in previous section was a very simple one that consisted in two conducting strips placed in close proximity. As a result of the coupling between the strips, when the structure was considered as a whole, a new collection of modes appeared. These new modes could be understood as a combination of the modes of the single rectangular strip flowing with the same phase, or with opposite phase, in both strips.

Generally, modes in coupled resonators can be classified in antenna, or transmission-line modes, depending on the kind of coupling they exhibit. Antenna modes can be identified by its currents flowing in phase in the coupled resonators. Conversely, in transmission-line modes the currents flow with a phase difference of $180^{\circ}$ between the elements. As it will be demonstrated next, antenna modes are efficient radiators, whereas transmission-line modes are characterized by poor radiating performance.

Then, it seems that a good understanding of the coupling phenomena that occur between multiple resonators could be very helpful when designing wideband structures. Consequently, this section is going to be focused on analyzing the coupling phenomena between coplanar and parallel planar resonators, in order to determine how to use coupling to increase the antenna impedance bandwidth.

\subsubsection{Coupling phenomena in coplanar plates}

The characteristic modes of a rectangular plate in free space have already been studied in section 5.2.1. Now, Figure 5.22 illustrates how characteristic modes behave when two rectangular plates are placed side-by-side. The plates, which are in free space, are identical, with length $L_{l}=2.8 \mathrm{~cm}$ and width $W_{1}=4$ $\mathrm{cm}$. The edge-to-edge separation between them is $0.4 \mathrm{~cm}$. It is observed that now, there are two horizontal current modes, $J_{1}$ and $J_{1}$, , and two vertical current modes, $J_{2}$ and $J_{2}$ '. This duplication of modes is due to the different coupling that occurs at the edge-to-edge separation. Drawing an analogy with the modes of a folded dipole, modes $J_{1}$ and $J_{2}$ in which current flows in phase in both plates, are called antenna modes. Following the same sort of criterion, modes $J_{l}$ ' and $J_{2}$ ', in which the current flows with $180^{\circ}$ phase difference in the two plates, are called transmission-line modes. It is expected that when the two plates are coupled in a transmission-line way, the total radiation will diminish because of the cancellation of the currents. This cancellation increases when currents are intense, and flow parallel to the edge-to-edge separation, like in vertical mode $J_{2}^{\prime}$. 
Figure 5.23 represents the variation with frequency of the characteristic angles associated to the previously described current modes. The observation of the slope of characteristic angle curves confirms that antenna modes, $J_{I}$ and $J_{2}$, present good radiating behaviour and broad bandwidth. The horizontal transmission-line mode, $J_{1}$ ' still presents quite an acceptable radiating bandwidth, while the vertical transmission-line mode $J_{2}$ ', in which the current cancellation is higher, presents poorer radiating characteristics than the rest of modes.

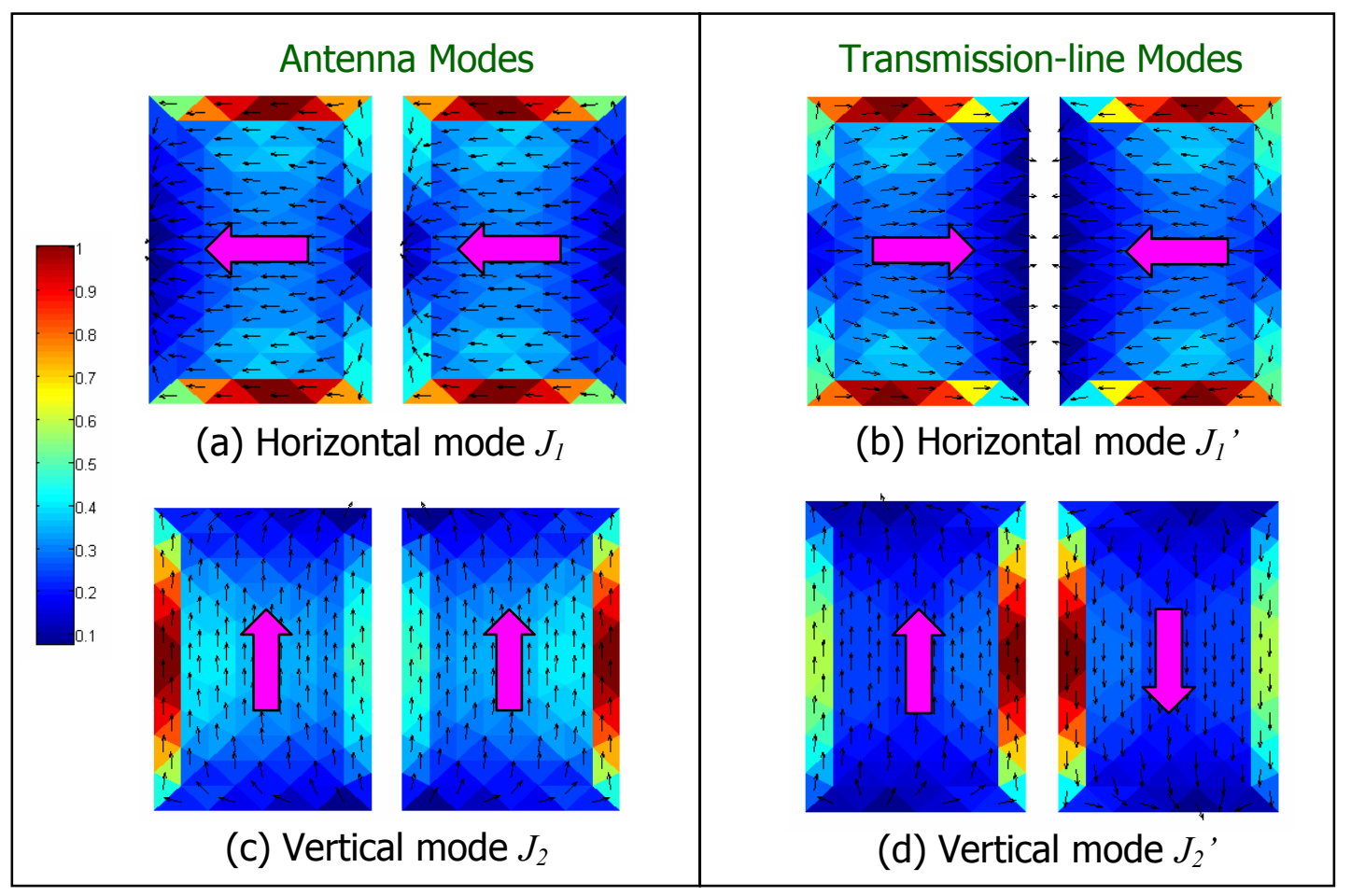

Figure 5.22 Normalized current distribution at $2.4 \mathrm{GHz}$ of the horizontal and vertical current modes of two identical plates placed close together.

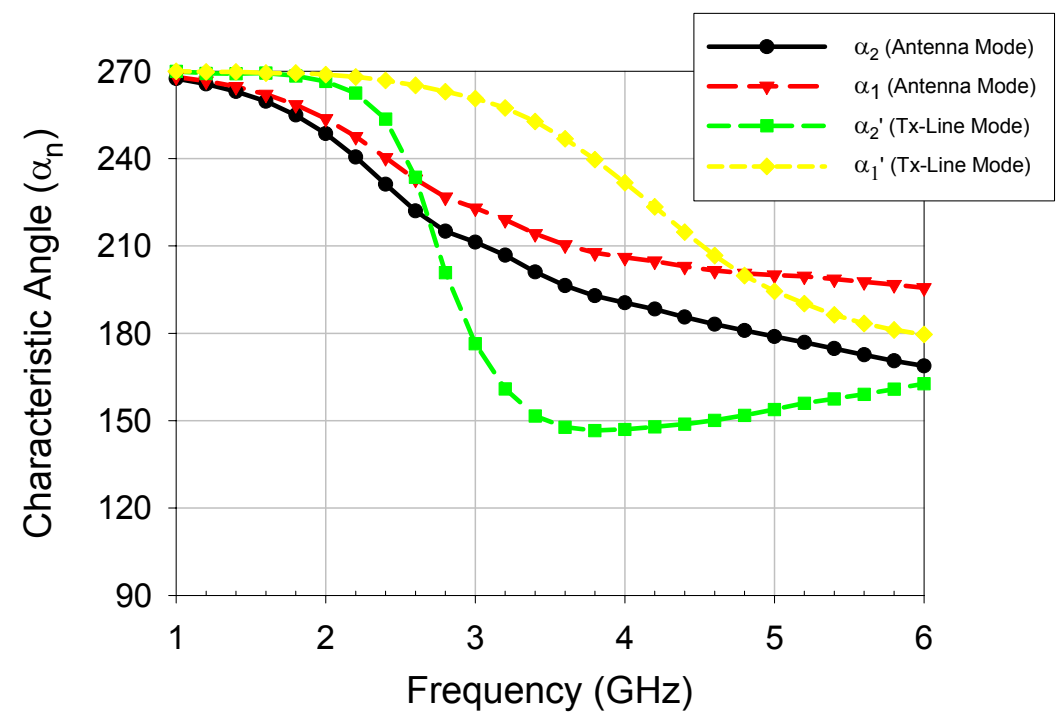

Figure 5.23 Characteristic angle variation with frequency for the current modes of Figure 5.21. 
Using different approaches, other authors have arrived at similar conclusions regarding the existence of different modes in coupled resonators, and its radiating properties. For example, in [109] the identification of the modes of two-coupled strip resonators is done through the distribution of voltages on the interface between the strips, and the input impedance. At first resonance, they infer that an even mode (antenna mode) is excited, since the phase difference between the voltages on the strips is nearly zero. Conversely, the mode at second resonance is identified as an odd mode (transmission-line mode), because of nearly $180^{\circ}$ phase difference between the strips. In [109] it is also stated that the radiated power is expected to be bigger for the even mode because of the in-phase excitation of the strips.

From these conclusions, it may be noted that when analyzing antennas based on the use of multiple resonators, the existence of antenna and transmissionline modes, should be taken into consideration. Classical applications are the use of parasitic elements placed next to a planar radiating element, or the design of planar reflectarrays. As a general rule, to obtain wide impedance bandwidth in coupled structures the excitation of antenna modes should be enhanced, while the excitation of transmission-line modes should be avoided. Sometimes this can be accomplished by choosing a proper feeding arrangement capable of preventing the creation of transmission-line currents.

Nevertheless, as the number of coupled elements increases also does the complexity of the modal analysis. For instance, Figure 5.24 shows the current distribution of the collection of horizontal current modes that appear in four coupled rectangular plates. As observed, now the number of horizontal modes multiplies by four. There is only one antenna mode characterized by currents flowing in-phase in the four plates. The rest of modes can be considered transmission-line modes, in which there is a different amount of current cancellation depending on the intensity and location of the transmission-line currents. For example, the first transmission-line mode experiments a strong coupling in the gap between the upper and lower plates, so it would suffer from strong current cancellation. In the second transmission-line mode the coupling occurs at the vertical gap between the left and right plates, and although currents flow with opposite phases, they do not cancel as much as in the first mode. Finally, in the third transmission-line mode, there are coupling effects at every gap between plates, so the mode is expected to be quite an inefficient radiator.

Figure 5.24 just constitutes a particular example of how the number of horizontal modes multiplies by four when four coupled plates are analyzed. However, it could be demonstrated that, all the modes of the rectangular plate presented in Figure 5.1 experiment similar coupling phenomena when the rectangular plate is placed close to other radiating elements. 


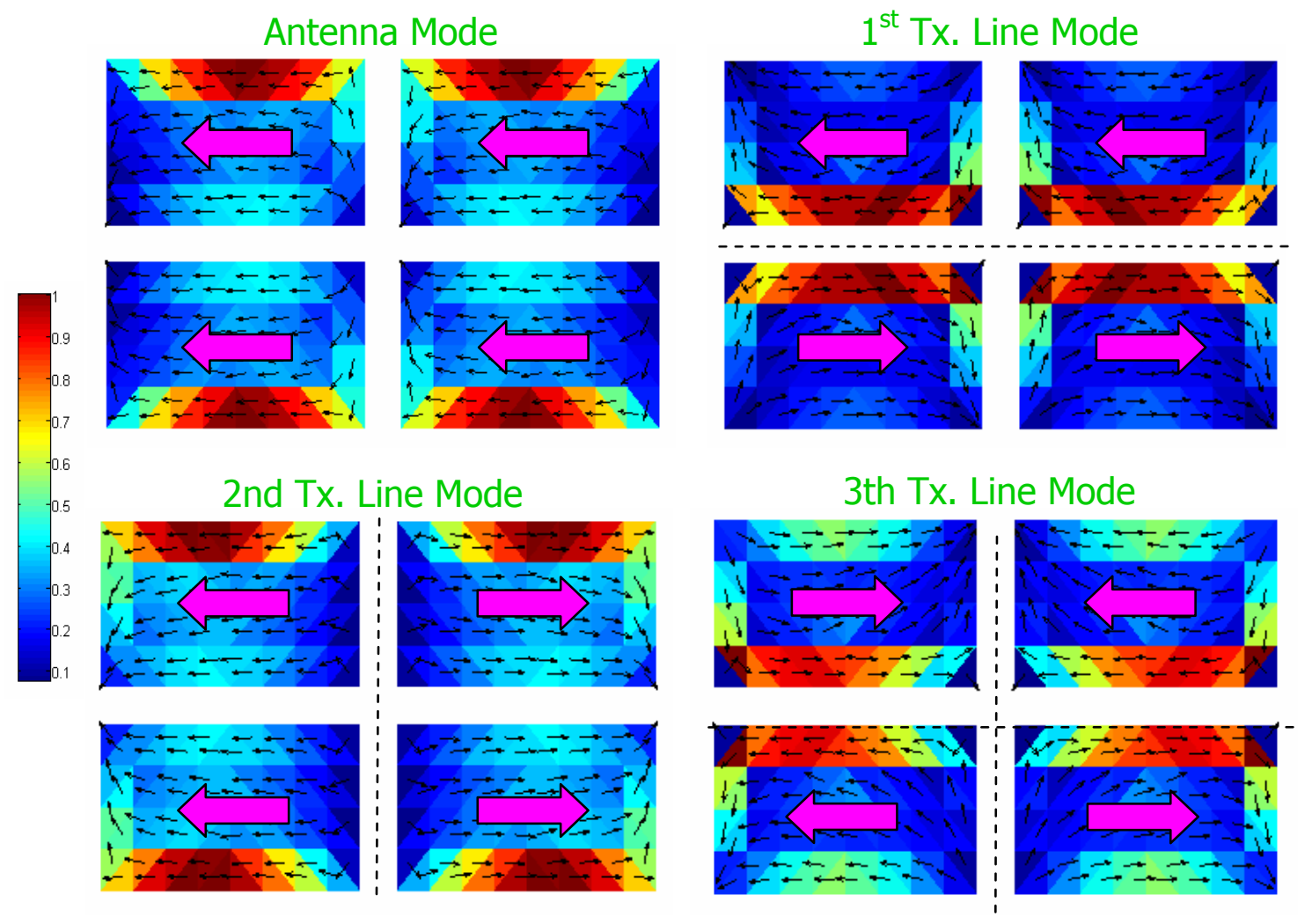

Figure 5.24 Current distribution of the horizontal current modes that appear as a result of the coupling phenomena between four rectangular plates.

Typically, structures formed by multiple resonators are used to obtain multiple frequency operation, or dual polarization. As a way of example, Figure 5.25 presents a wideband planar antenna formed by four coupled circular plates, which employs multiple feeding to provide multiple polarizations [118]. The antenna can also be considered as two planar circular dipoles. To avoid the excitation of transmission-lines modes, a correct choice of the polarity of voltage generators must be made. Figure 5.26 presents the normalized current distribution at $2 \mathrm{GHz}$ for the horizontal and vertical antenna modes of the structure. Horizontal current mode $J_{I}$ will be excited yielding horizontal polarization, if the antenna is symmetrically fed by voltage generators $V_{3}$ and $V_{4}$. Likewise, if voltage generators $V_{1}$ and $V_{2}$ are used, the excited mode will be the vertical current mode $J_{2}$, which will provide vertical polarization. Finally, feeding simultaneously with the four voltage generators, linear polarization at $\pm 45^{\circ}$ can be achieved, as a result of the combined excitation of degenerated modes $J_{1}$ and $J_{2}$.

At last, the wideband performance of the double dipole antenna can be checked in Figure 5.27, which shows the VSWR for $50 \Omega$ either when the antenna is fed simultaneously by voltage generators $V_{1}$ and $V_{2}$, or by $V_{3}$ and $V_{4}$. 


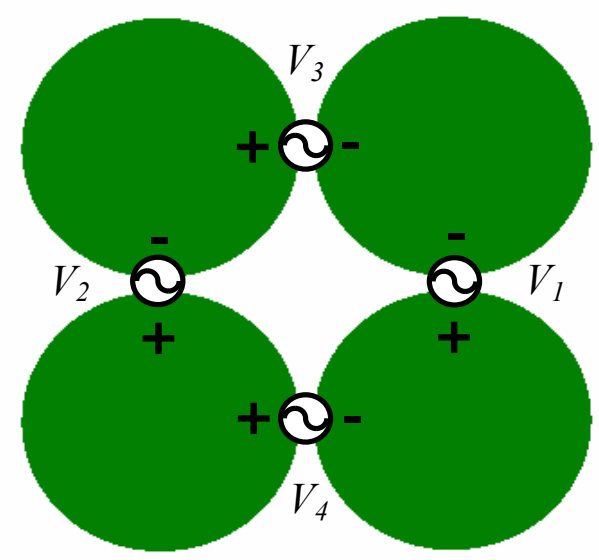

Figure 5.25 Wideband planar antenna formed by four coupled circular plates, which employs multiple feeding to provide multiple polarizations.

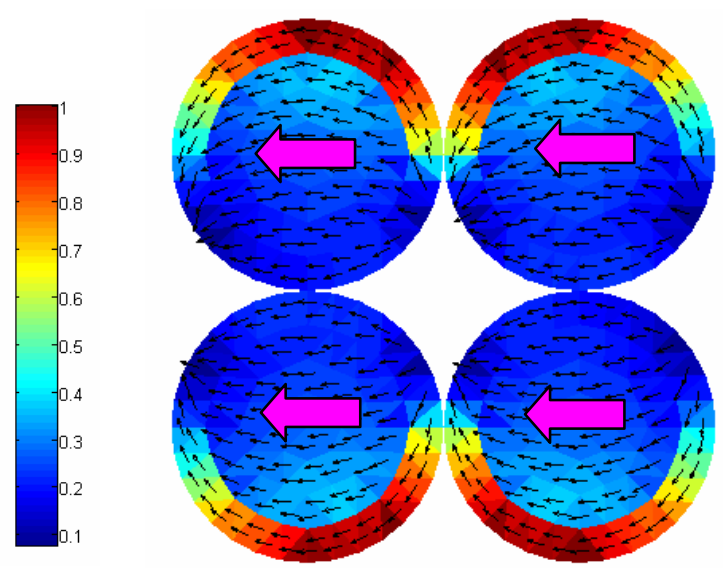

Horizontal antenna mode $J_{l}$

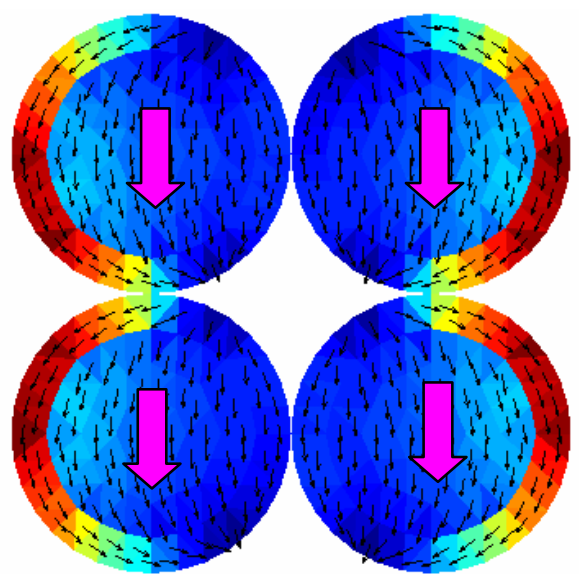

Vertical antenna mode $J_{2}$

Figure 5.26 Normalized current distribution at $2 \mathrm{GHz}$ of the horizontal and vertical antenna modes.
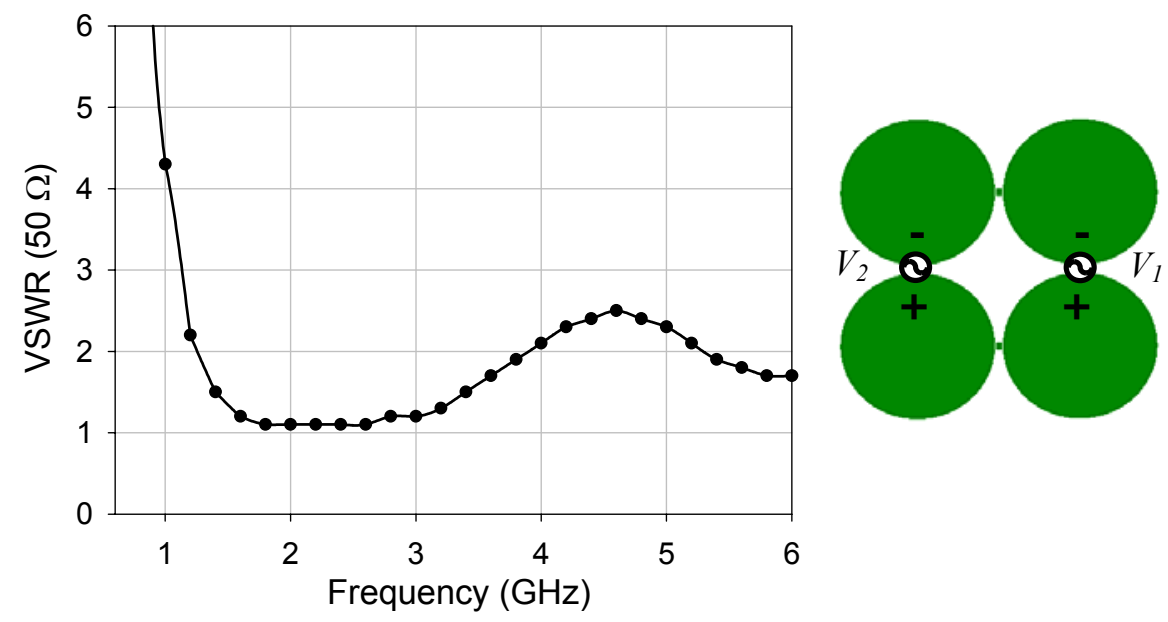

Figure 5.27 VSWR for $50 \Omega$ when the antenna is fed in parallel by voltage generators $V_{1}$ and $V_{2}$. 


\subsubsection{Coupling phenomena in parallel plates}

The coupling phenomena already described for coplanar plates are also present at parallel plates, so modes existing in coupled parallel structures can be once more classified in antenna modes and transmission-line modes. Figure 5.28 depicts the current distribution for horizontal and vertical current modes of a set of two parallel rectangular plates at $1 \mathrm{GHz}$. The dimensions of each plate are $W=4 \mathrm{~cm}$ and $L=6 \mathrm{~cm}$. The distance between plates is $h=1.5 \mathrm{~cm}$. The characteristic angle curves shown in Figure 5.29 can be used to determine the radiating properties of each mode. As expected, antenna modes present softer slope and hence, better radiation properties and wider bandwidth than transmission-line modes. Note however that due to the strong capacitive coupling between both plates, characteristic angle curves are shifted upwards, so antenna modes exhibit capacitive contribution $\left(\alpha_{n}>180^{\circ}\right)$ at every frequency. Actually, due to this shifting, the vertical antenna mode $J_{2}$ does not resonate in the studied frequency band.

In order to illustrate that mode duplication happens with every mode, Figure 5.30 shows de current distribution at $1 \mathrm{GHz}$ for the first antenna and transmission-line higher order modes of the set of two parallel rectangular plates. As observed, both higher order modes exist in its antenna and transmission-line version, $J_{3}$ and $J_{4}$, and, $J_{3}$ ' and $J_{4}{ }^{\prime}$, respectively.

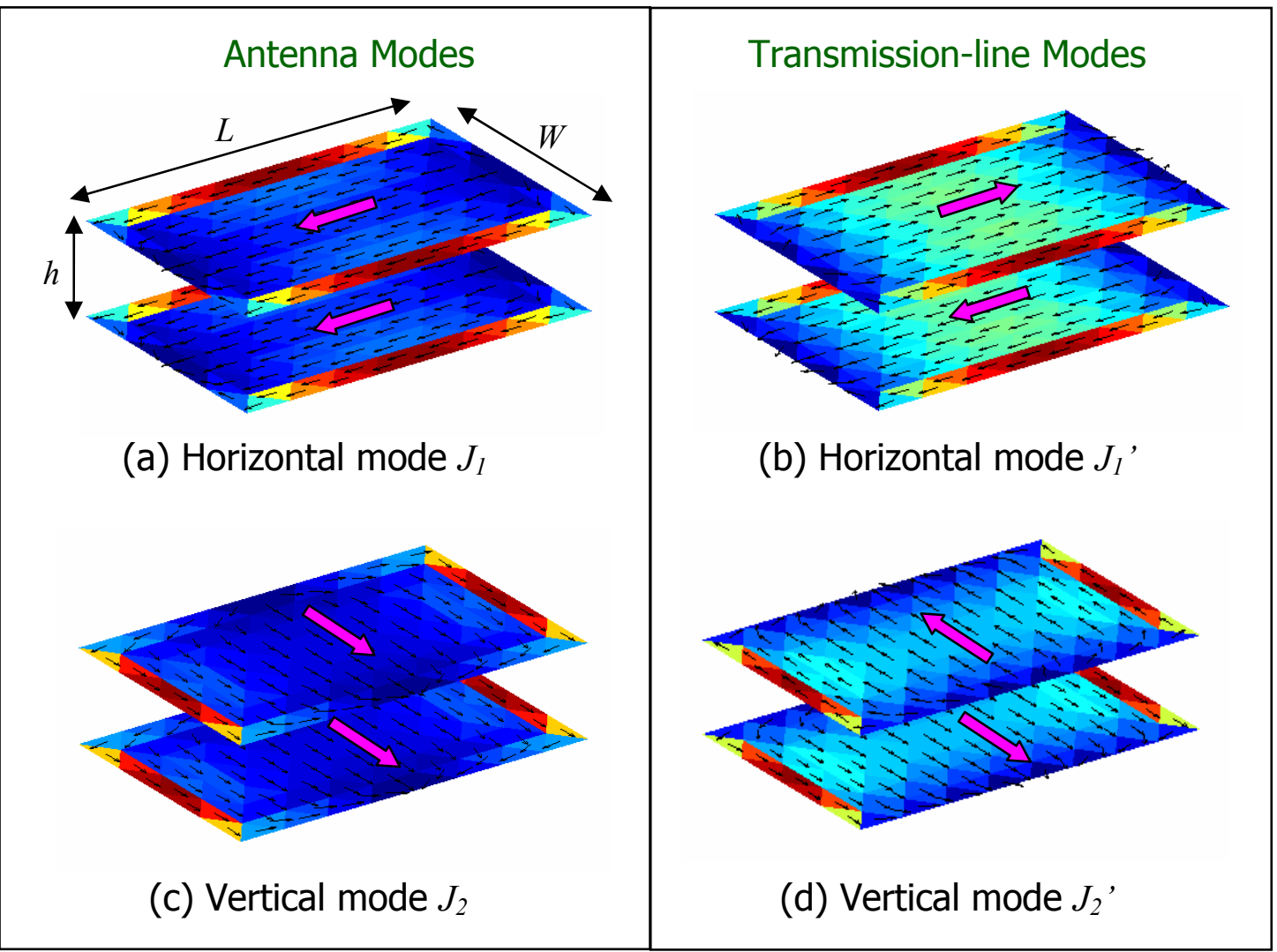

Figure 5.28 Normalized current distribution at $1 \mathrm{GHz}$ for the horizontal and vertical current modes $J_{1}$ and $J_{2}$ of a set of two parallel rectangular plates. 


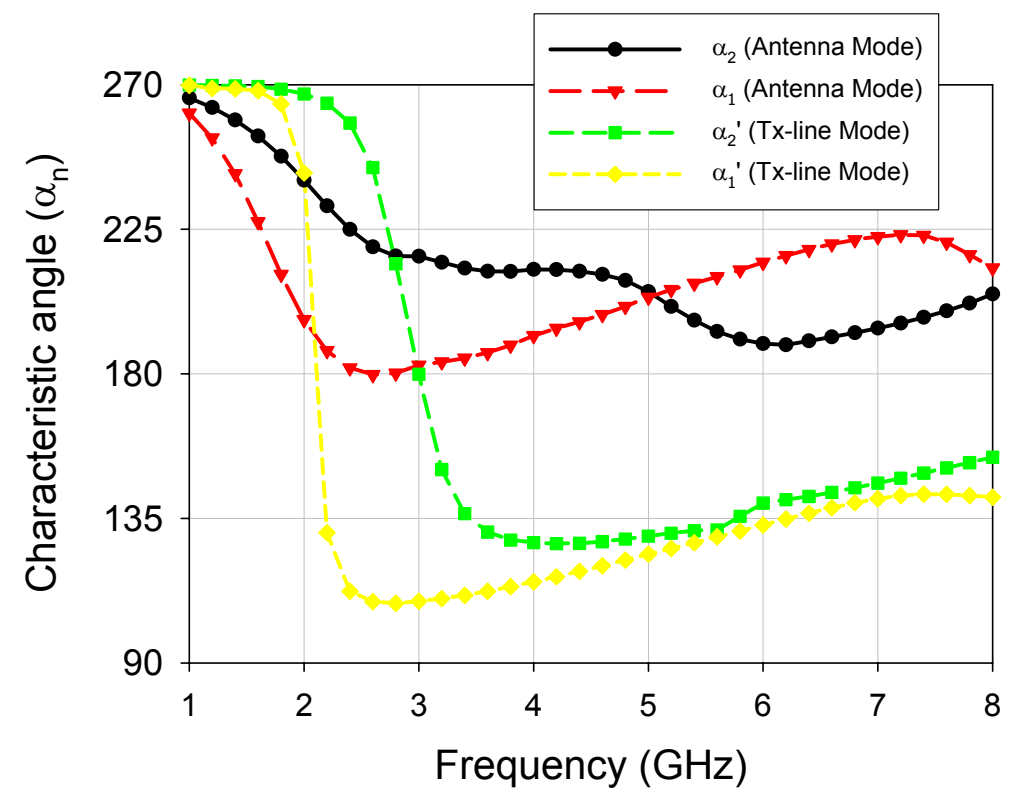

Figure 5.29 Characteristic angle variation with frequency for the current modes of Figure 5.27.

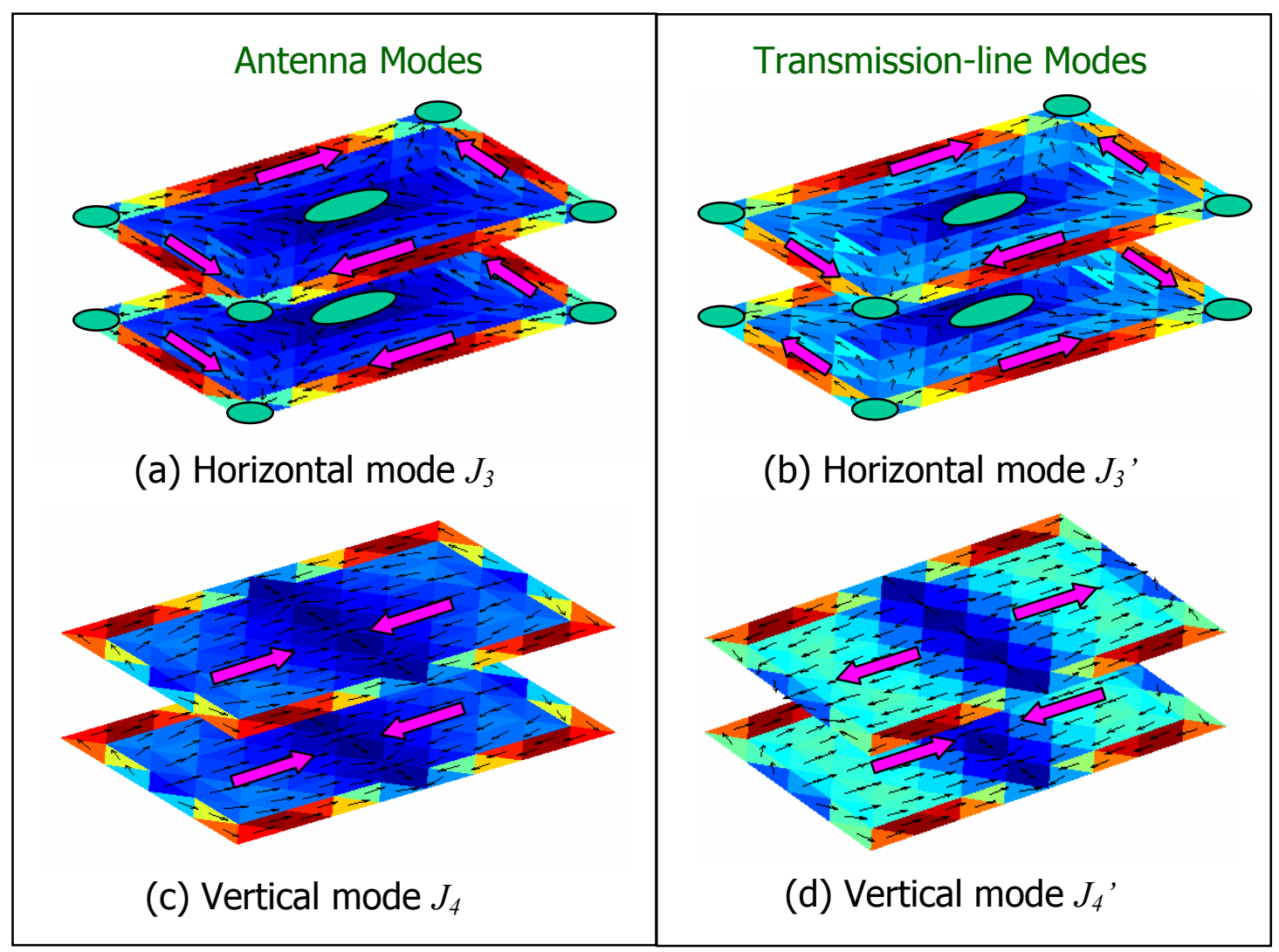

Figure 5.30 Normalized current distribution at $1 \mathrm{GHz}$ for higher order current modes $J_{3}$ and $J_{4}$ of a set of two parallel rectangular plates. 
Antenna and transmission-line modes should be taken into account when designing antennas formed by parallel radiators, like stacked patches, or patches mounted above a finite ground plane. Figure 5.31 presents an example of the current distribution at $2 \mathrm{GHz}$ for the first modes of a rectangular patch of dimension $2 \mathrm{~cm} \times 4 \mathrm{~cm}$, mounted $0.75 \mathrm{~cm}$ above a finite ground plane $(10 \mathrm{~cm} \times$ $10 \mathrm{~cm}$ ), with air dielectric. As it can be observed, although the patch and the plane are connected with a strip, the current distribution of modes is very similar to that obtained for the parallel plates analyzed above. This means that all the results previously presented can be applied to the analysis of radiating patches over finite ground planes.
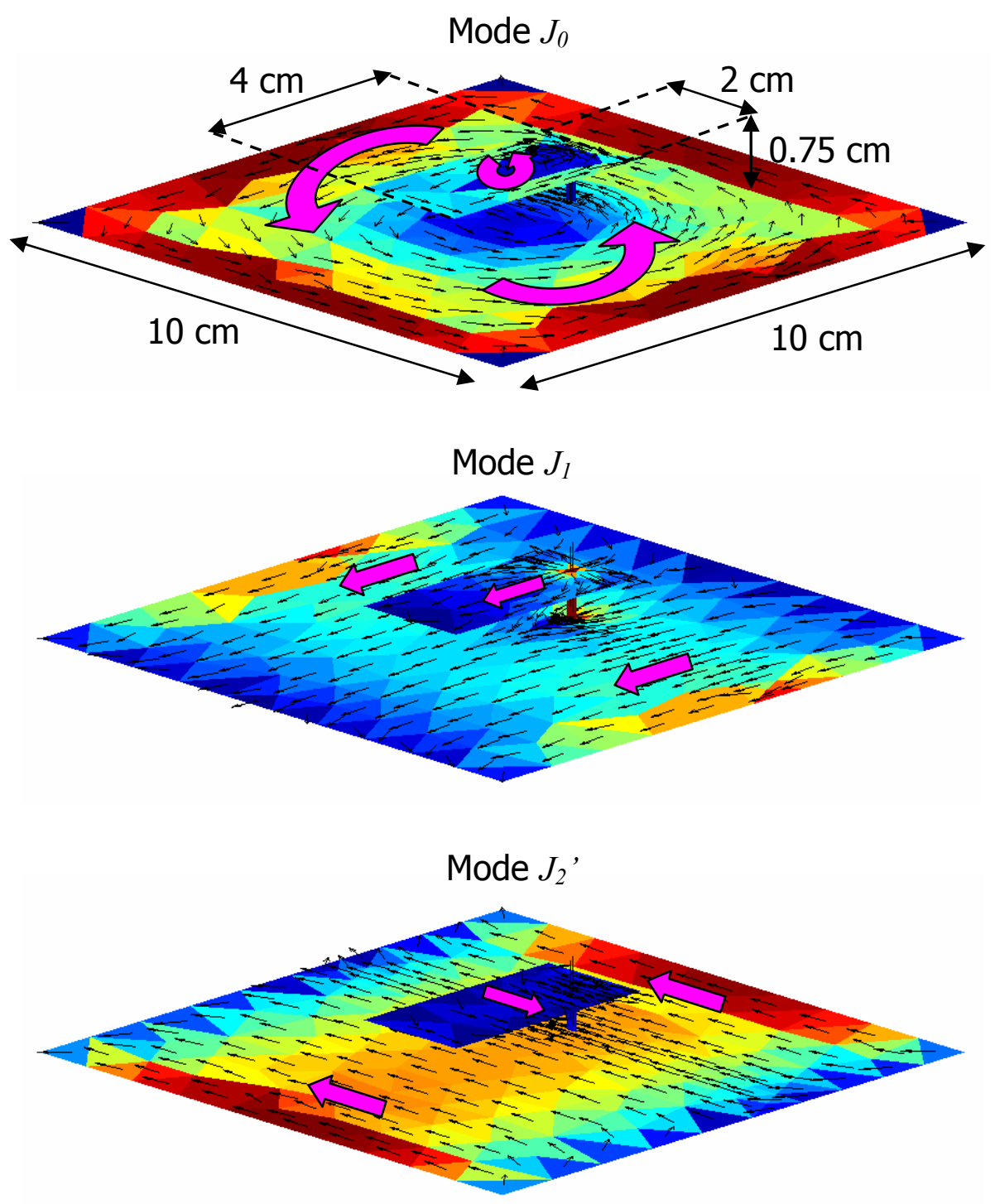

Figure 5.31 Normalized current distribution of the first modes of a rectangular patch over finite ground. 


\subsection{Modal analysis of planar monopoles}

Planar monopoles are very well-known antennas that have long been used in mobile communications due to their remarkable properties such as wide impedance bandwidth, omnidirectional radiation pattern, simple structure, small size, and low cost. Because of the broad bandwidth they provide, planar monopole antennas are extremely attractive to be used in emerging ultrawideband applications [104]. Moreover, they are also considered excellent candidates to face up the increasing demand for wireless communication services, which require multi-band or broadband antennas capable of operating at different standards.

In last years, a lot of work has been focused on the determination of the planar monopole shape which provides the wider impedance bandwidth. As a result, a great number of different planar monopole geometries have been characterized experimentally [119]-[123], and automatic design methods have been developed to achieve the optimum planar shape [124]. Moreover, other strategies to improve the impedance bandwidth which do not involve a modification of the geometry of the planar element have been investigated. Basically, these strategies consist of adding a shorting pin to the structure [125]-[127] or using multiple feeding points to excite the antenna [128].

Therefore, a great variety of experiments related to planar monopole geometries and feeding configurations have been carried out, but due to the lack of an analytical model, very little analysis on the physical understanding of the operating behaviour of monopoles has been considered up to now.

The main purpose of this section is to perform a modal analysis of various planar monopole geometries in order to acquire a clear knowledge of their operating principle. In the first instance, it will be demonstrated that the shape of the monopole in the feeding gap zone is very critical, as it determines the bandwidth performance of the monopole. In general, the impedance bandwidth of a monopole can be increased by bevelling its base appropriately. On the contrary, the upper part of the monopole is not so important, as it only acts as a load, and it does not affect very much the matching of the antenna.

Next, using characteristic modes it will be proved that feeding arrangements that preserve the symmetry of the monopole, avoid the excitation of modes with transverse currents, and reduce the cross polarization levels. Conversely, nonsymmetrical feedings enhance the excitation of transverse modes, and yield wider bandwidth than centre-feedings when all modes contributing to radiation present similar resistance values. Finally, the effect of the insertion of a shorting pin, and the improvement obtained when using multiple feeding points, will also be explained in terms of characteristic modes. 


\subsubsection{Influence of the shape of planar monopoles in the impedance bandwidth performance.}

The most commonly used planar monopole shapes are square, rectangular, triangular, circular, elliptical, and pentagonal. Figure 5.31 shows some of these monopole geometries. Among them, the circular monopole and the elliptical monopole fed along the major axis, have been reported to yield maximum bandwidth [102]. However, it has been demonstrated that although the square monopole provides smaller bandwidth than the circular monopole, its radiation pattern suffers less degradation within the impedance bandwidth [120]. As will be shown next, the different impedance bandwidth of the circular and the square monopole can been explained using characteristic modes.
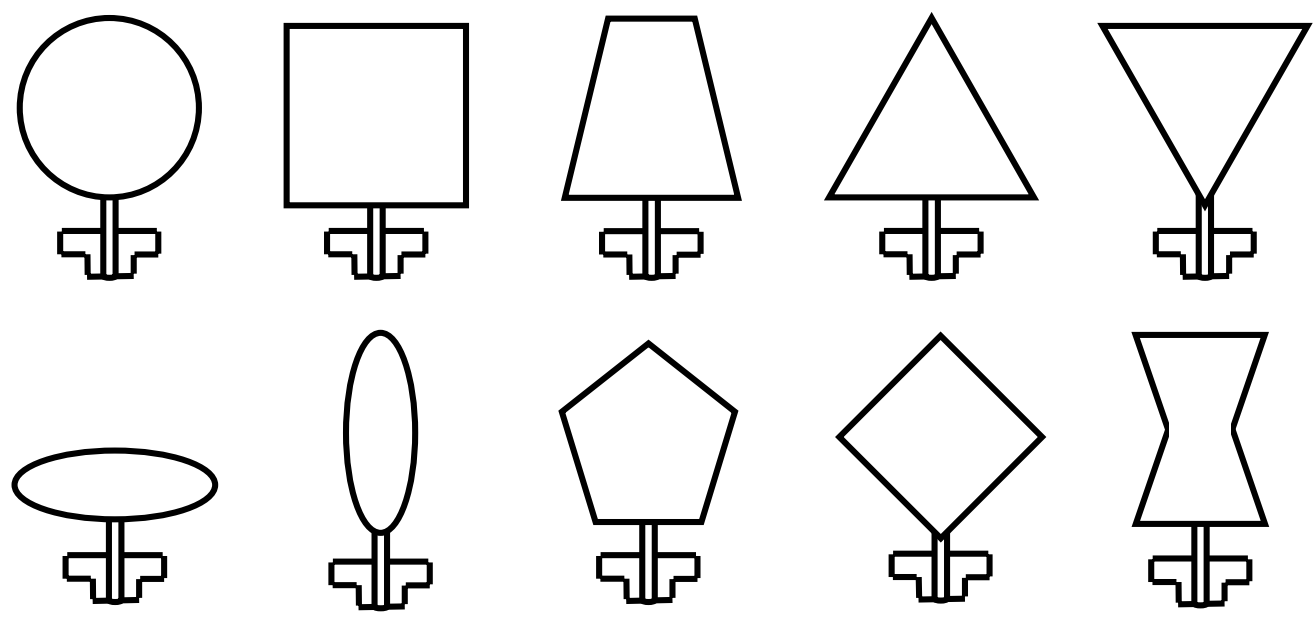

Figure 5.32 Most commonly used planar monopole shapes.

In order to find out how the shape of planar monopoles affects its impedance bandwidth performance, a modal analysis of four typical monopole geometries is going to be performed. The selected geometries, which are shown in Figure 5.33, are square, bowtie, reverse bowtie, and circular. For the shake of comparison, the height of all monopoles is $L=4 \mathrm{~cm}$, the feed gap distance is $h=2.5 \mathrm{~mm}$, and the ground plane presents infinite dimension, in all cases.

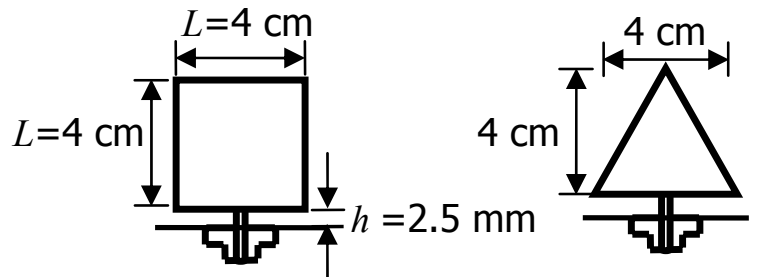

a) Square

b) Reverse bowtie

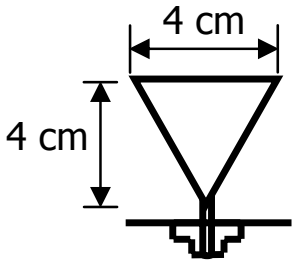

c) Bowtie

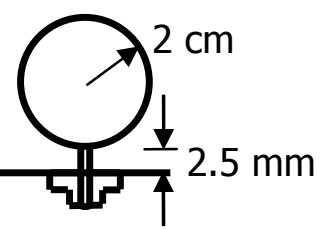

d) Circular

Figure 5.33 Planar monopole geometries to be compared. 
Figure 5.34 shows the normalized current distribution for the first characteristic modes of these planar monopoles. Mode $J_{0}$ is a special non resonant inductive mode whose currents form close loops. Mode $J_{1}$ presents vertical current flow along the monopoles, with intense currents at the feeding strip. In contrast, mode $J_{2}$ can be identified by horizontal currents flowing parallel to the ground plane. Mode $J_{3}$ is a higher order mode that, as mode $J_{l,}$, exhibits vertical currents. The difference between these two modes is that $J_{3}$ has a current null near the base of the monopoles, and intense currents on the side edges of the structures. An analysis of the variation with frequency of characteristic currents would reveal that this current null of mode $J_{3}$ moves forward the top of the monopole as the frequency increases. Finally, $J_{4}$ is a mixed current mode with vertical and horizontal currents. As observed, the current distribution of mode $J_{4}$ changes quite a lot with the monopole geometry.

As observed in Figure 5.34 modes $J_{0}, J_{2}$ and $J_{4}$, present low current intensity (blue colour) at the feeding strip in some monopole geometries. This means that this location of the feeding would not be optimum for these modes, which would be weakly coupled to a vertical feeding port placed at this strip. It should be remarked that for the case of the circular monopole, all modes exhibit quite a high current intensity at the feeding strip. This happens because the base of the circular monopole acts as a soft transition between the feeding strip and the monopole, so it improves the matching of modes with transverse current components.

Next, the radiating behaviour of the modes for the different monopoles is going to be compared by means of characteristic angle curves. Figure 5.35 presents the characteristic angle variation with frequency for the first four radiating modes of the monopoles. As observed, mode $J_{l}$ exhibits similar radiating behaviour and resonant frequency in all the monopoles. This happens because the resonance of mode $J_{1}$ depends on the height of the monopoles, which is the same in all cases. In contrast, the resonant frequency and radiating properties of modes $J_{2}, J_{3}$, and $J_{4}$, differ quite a lot from one shape to the other.

Table 5.2 summarizes the resonant frequencies for the first three radiating modes of the monopoles. Mode $J_{4}$ has not been included in the table since it presents a resonant frequency higher than $8 \mathrm{GHz}$, in all the monopoles except in the square one. Table 5.3 compares the modal quality factors computed using (2.29), and Table 5.4 presents the modal radiating bandwidths that have been obtained by inversion of the modal quality factors. For the case of mode $J_{3}$ of the square monopole, and modes $J_{2}$ and $J_{3}$ of the circular monopole, the modal quality factor is very small, so the approximation $B W_{n}=1 / Q_{n}$ fails. On the base of the information yield by the previous tables, it can be concluded that the circular monopole seems to be the most efficient radiating structure. However, the final contribution of each mode to the total radiation of the antenna will depend on the antenna feeding. 
MODE $J_{0}(1 \mathrm{GHz})$
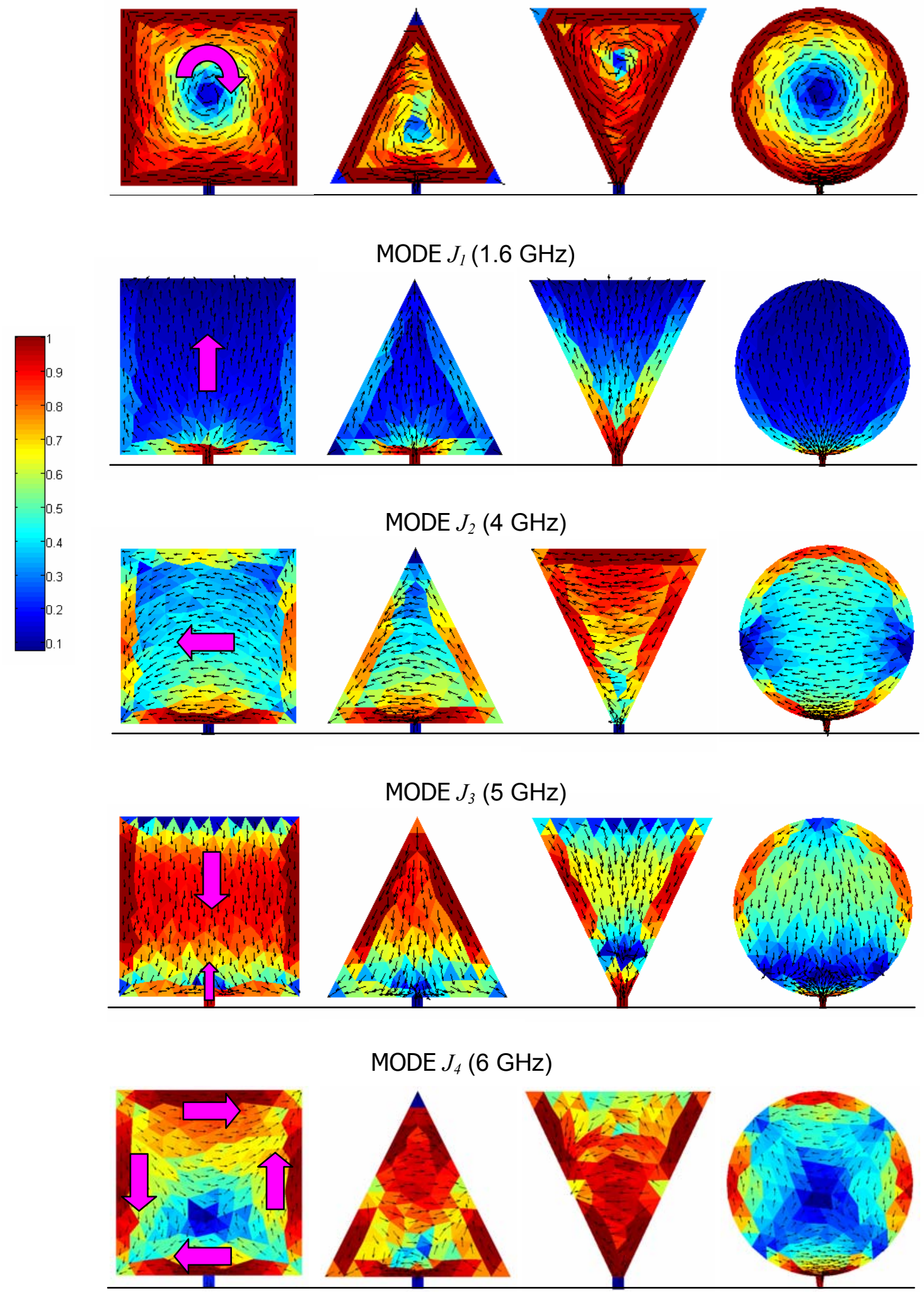

Figure 5.34 Normalized current distribution for the first five modes of different planar monopole geometries. 


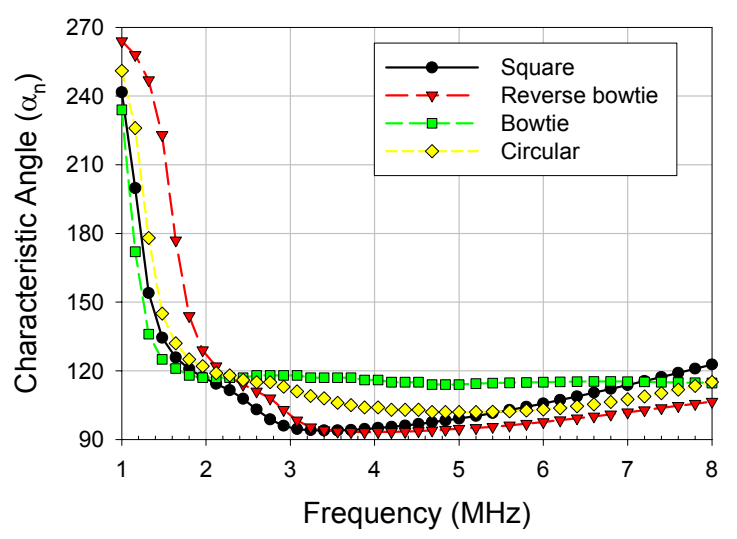

(a) Mode $J_{1}$

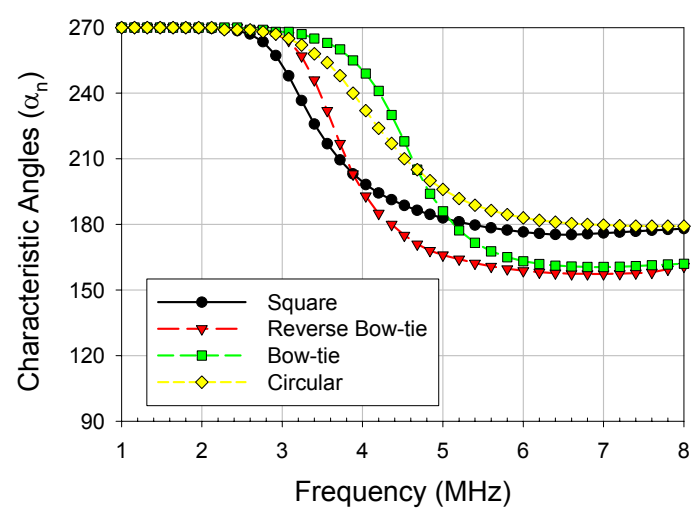

(c) Mode $J_{3}$

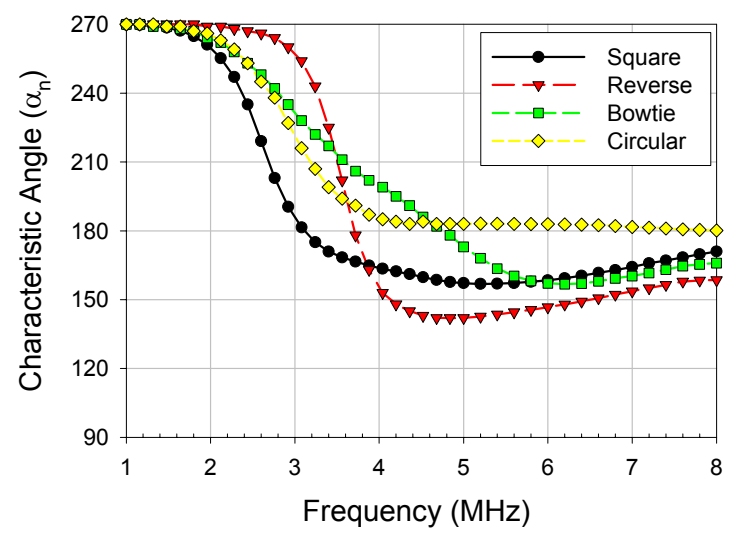

(b) Mode $J_{2}$

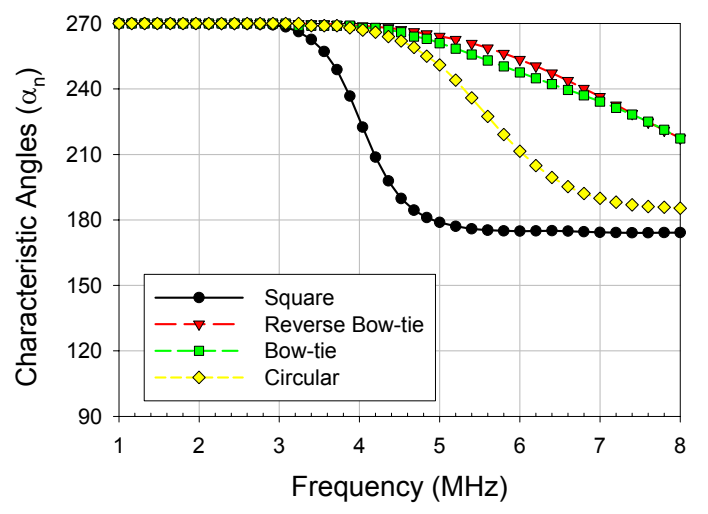

(d) Mode $J_{4}$

Figure 5.35 Characteristic angle variation with frequency for the first three radiating modes of a square, reverse bowtie, bowtie and circular monopole.

Resonant frequency: $f_{\text {res }}$

\begin{tabular}{|c|c|c|c|}
\cline { 2 - 4 } \multicolumn{1}{c|}{} & Mode $J_{1}$ & Mode $J_{2}$ & Mode $J_{3}$ \\
\hline Square & $1.20 \mathrm{GHz}$ & $3.10 \mathrm{GHz}$ & $5.30 \mathrm{GHz}$ \\
\hline Reverse bowtie & $1.60 \mathrm{GHz}$ & $3.70 \mathrm{GHz}$ & $4.35 \mathrm{GHz}$ \\
\hline Bowtie & $1.10 \mathrm{GHz}$ & $4.75 \mathrm{GHz}$ & $5.15 \mathrm{GHz}$ \\
\hline Circular & $1.30 \mathrm{GHz}$ & $8.00 \mathrm{GHz}$ & $6.75 \mathrm{GHz}$ \\
\hline
\end{tabular}

Table 5.2. Resonant frequencies for the first three modes of different planar monopole geometries.

Modal quality factor: $Q_{n}$

\begin{tabular}{|c|c|c|c|}
\cline { 2 - 4 } \multicolumn{1}{c|}{} & Mode $J_{1}$ & Mode $J_{2}$ & Mode $J_{3}$ \\
\hline Square & 3.17 & 1.09 & 0.34 \\
\hline Reverse bowtie & 4.14 & 5.10 & 1.19 \\
\hline Bowtie & 5.20 & 1.04 & 1.97 \\
\hline Circular & 3.52 & 0.13 & 0.12 \\
\hline
\end{tabular}

Table 5.3. Modal quality factor for the first three modes of different planar monopole geometries. 
Modal radiating bandwidth: $B W_{n}$

\begin{tabular}{|c|c|c|c|}
\cline { 2 - 4 } \multicolumn{1}{c|}{} & Mode $J_{1}$ & Mode $J_{2}$ & Mode $J_{3}$ \\
\hline Square & $31.54 \%$ & $91.74 \%$ & $>100 \%$ \\
\hline Reverse bowtie & $24.15 \%$ & $19.60 \%$ & $84.10 \%$ \\
\hline Bowtie & $19.23 \%$ & $96.15 \%$ & $50.76 \%$ \\
\hline Circular & $28.40 \%$ & $>100 \%$ & $>100 \%$ \\
\hline
\end{tabular}

Table 5.4. Modal radiating bandwidth for the first three modes of different planar monopole geometries.

Now, monopoles are going to be fed with a voltage generator placed at the vertical strip. As it was demonstrated in (2.32), the total input admittance of an antenna $Y_{\text {in }}$ can be expanded in terms of complex modal admittances $Y_{n}=G_{n}+j B_{n}$. The visualization of the variation with frequency of modal admittances helps to identify which modes are excited.

Figure 5.36 depicts the contribution of modal admittances to the total input admittance of the different planar monopoles. As observed, the voltage difference created at the vertical strip favours only the excitation of vertical current modes, $J_{1}$ and $J_{3}$. In all geometries, the fundamental mode $J_{1}$ is dominant at lowest frequencies, and forces the first resonance. Conversely, the resonance of mode $J_{3}$ is only present in the bowtie monopole. Two different behaviours are observed regarding the interaction of modes. On one hand, at the square, reverse bowtie, and circular monopole, the antiresonance that results from the combination of modes $J_{1}$ and $J_{3}$, is very soft. On the other hand, in the bowtie monopole, the interaction of the inductive mode $J_{1}$ and the capacitive mode $J_{3}$, creates a sharp antiresonance at $2.45 \mathrm{GHz}$.

Alternatively, with the aim of determining if the modes are well matched to the feeding, modal voltage standing wave ratios $\left(V S W R_{n}\right)$ can be computed from the modal admittances $Y_{n}$. In Figure 5.37 the total $V S W R$ of every monopole has been plotted together with the modal $V S W R_{n}$.

From Figure 5.37 it is extracted that the square, and the reverse bowtie monopole present a single matched band, with an impedance bandwidth for $V S W R<2.0$ of $83 \%$ and $57 \%$, respectively. In both cases, mode $J_{1}$ imposes the lowest matched frequency of the band, while mode $J_{3}$ determines the highest matched frequency. A cursory examination of the modal admittances in Figure 5.36 reveals that for both the square and the reverse bowtie monopoles, the conductances $G_{1}$ and $G_{3}$ of modes $J_{1}$ and $J_{3}$, are close to $20 \mathrm{~m}^{-1}$ in a wide range of frequencies. The inversion of these modal conductances will provide equal well matched modal resistances that will yield a wide matched band. The similar admittance behaviour of the square and reverse bowtie monopole can be attributed to the identical not bevelled feeding gap configuration of both geometries. 
On the contrary, the bowtie monopole, which can be considered the planar form of the conical antenna, presents a continuous bevelling from the base to the top of the monopole, and exhibits two separate weakly matched bands. The first band is due to mode $J_{l}$, while the second band is a consequence of the interaction of modes $J_{1}$ and $J_{3}$. Note that although the influence of mode $J_{1}$ extends up to $8 \mathrm{GHz}$, the mode is not very well matched, since its modal conductance $G_{1}$ is very low at all frequencies except from $1.2 \mathrm{GHz}$ to $1.6 \mathrm{GHz}$. The matching of mode $J_{1}$ could be improved by reducing the bevelling angle, which would increase the modal conductance values.

Further discussion about the dependence of input impedance matching of the bowtie and the reverse bowtie monopoles, with the height and the bevelling angle can be found in [129].

With regard to the circular monopole, the smooth curvature of its base can be considered as a progressive bevelling. This soft bevelling acts as a broadband transition between the feeding strip and the monopole, so it improves the matching. In this case, the circular monopole provides the widest impedance bandwidth (93\% for $V S W R<2.0$ ). The good matching from $1.4 \mathrm{GHz}$ to $4.6 \mathrm{GHz}$ is mainly due to the contribution of mode $J_{1}$ to the total $V S W R$, while mode $J_{3}$, which is not well matched by its own, cooperates to improve the matching only at $3.8 \mathrm{GHz}$.

Note that the progressive bevelling of the circular monopole improves the matching of mode $J_{1}$, leading to a well matched mode in a broad frequency band. Nevertheless, mode $J_{3}$ is not altered by the bevelling as it presents a current null near the base of the monopoles, which creates a very low current at the feeding point.

To sum up, the feeding gap configuration at the base of the monopoles is crucial, as it affects the matching of modes, and in particular of mode $J_{l}$. By bevelling the base of the monopoles, mode $J_{1}$ transforms in a broadband matched mode, whose influence extends up to high frequencies. The bowtie monopole and the circular monopole are examples of monopoles with a bevelled base. For the case of the bowtie, the excessive bevelling results in two separate ill matched bands. The circular monopole presents a progressive bevelling that creates a soft transition from the feeding strip to the radiating element. This effect favours the radiation and improves the coupling between the radiating element and the feeding strip.

As will be demonstrated in next sections, any alteration in the symmetry of the monopoles, like a non-centred feeding strip, or the introduction of a shorting pin in one of the corners, would result in the excitation of transverse modes, like mode $J_{2}$. The excitation of these modes may increase the impedance bandwidth at the cost of ruining the polarization purity of the antenna. But, before going to next sections, let us present another example in order to illustrate the role played by the upper part of planar monopoles. 
Square monopole
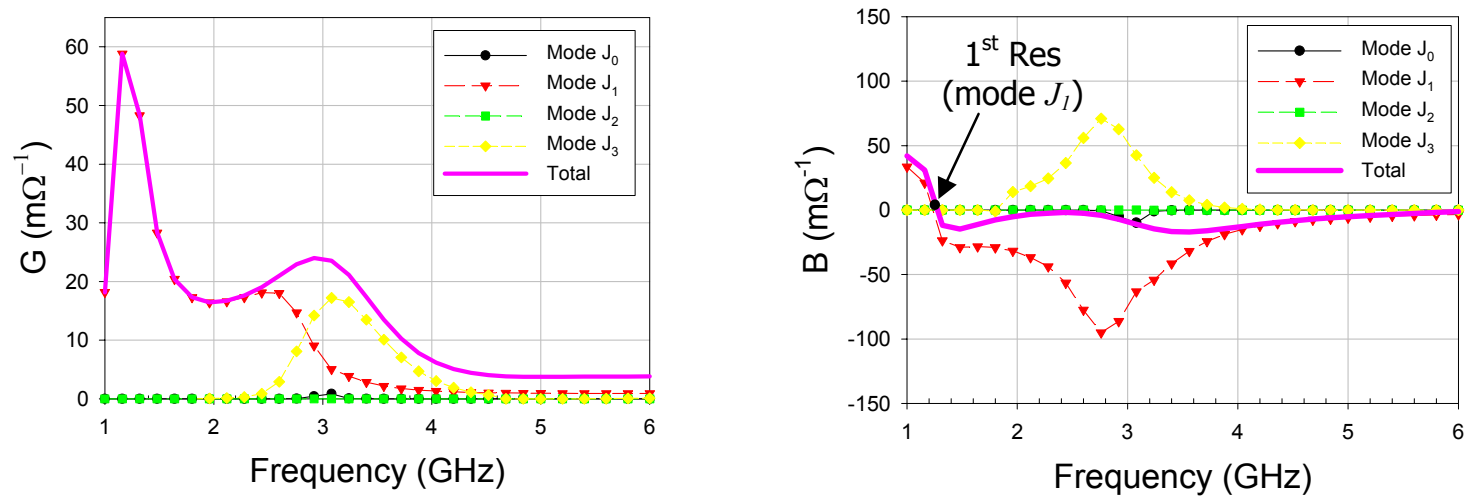

Reverse bowtie monopole
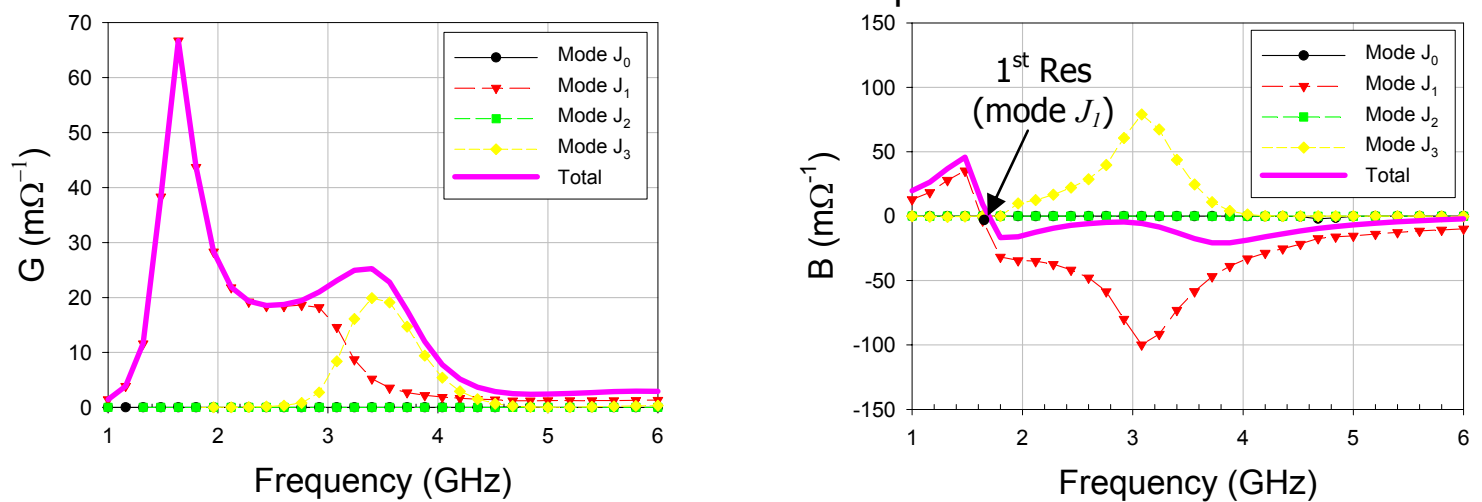

Bowtie monopole
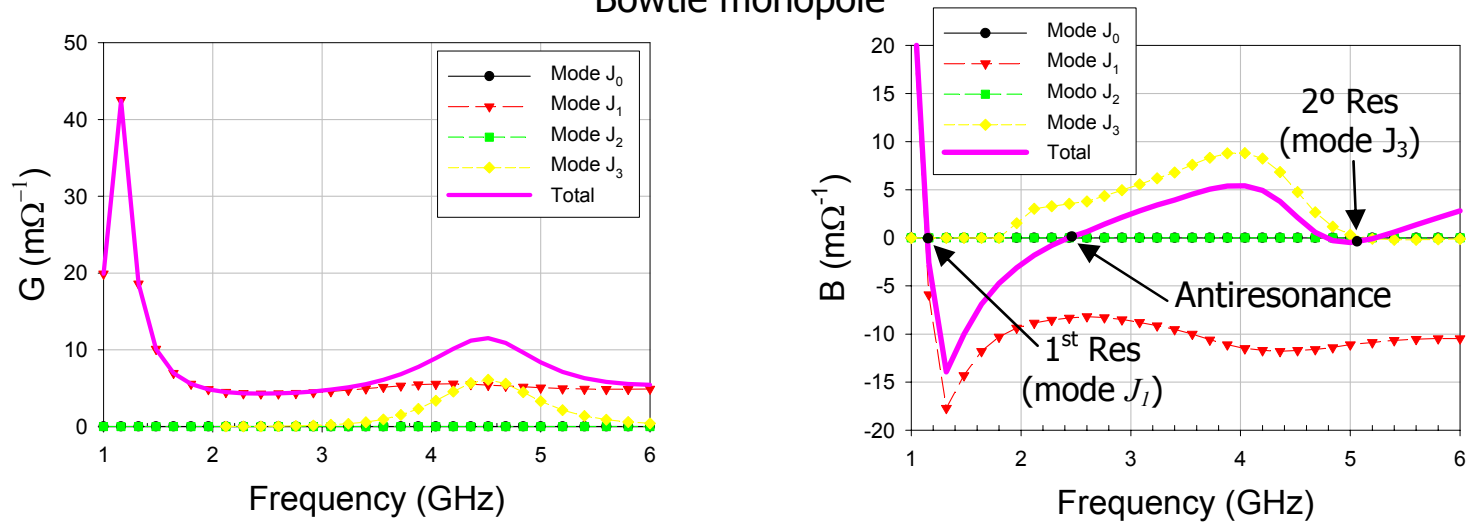

Circular monopole
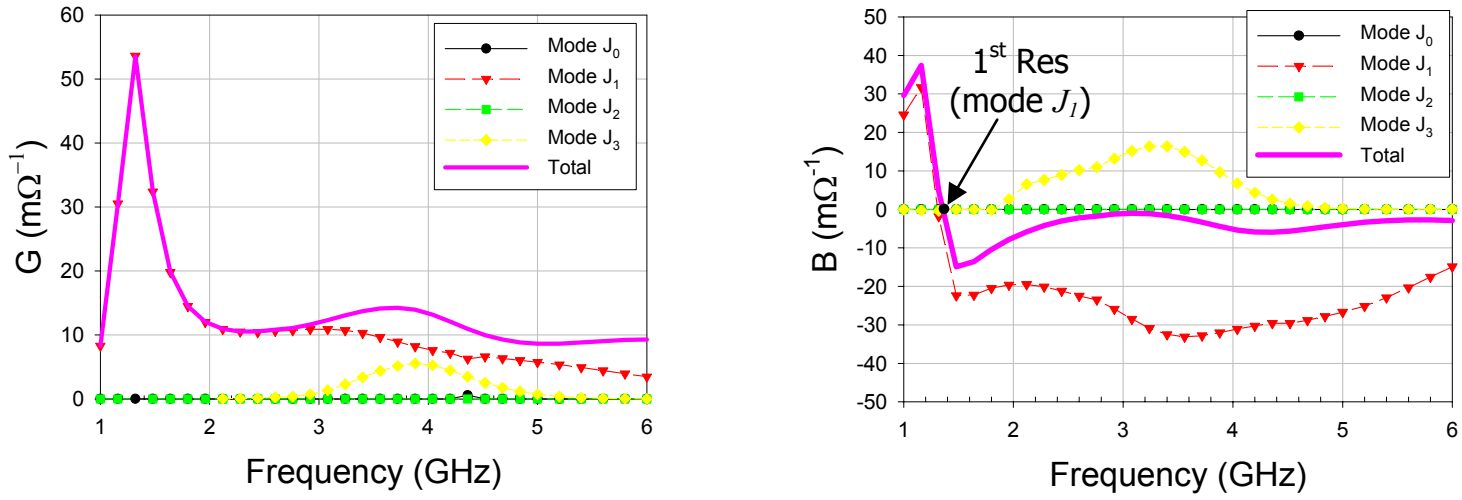

Figure 5.36 Contribution of modal admittances $Y_{n}$ to the total input admittance $Y_{\text {in }}$ of different planar monopole geometries. 

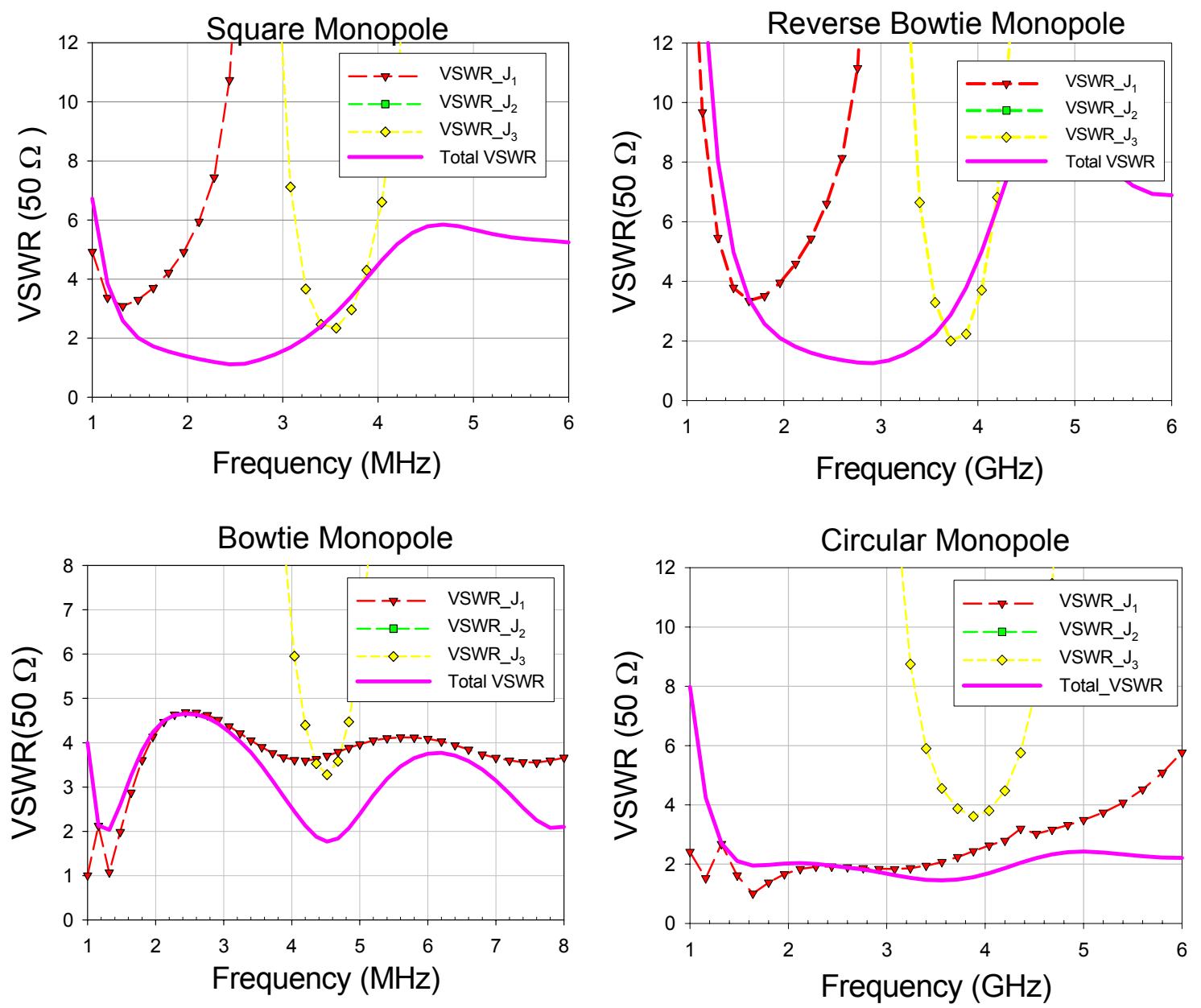

Figure 5.37 Contribution of the VSWR of modes to the total VSWR of different planar monopoles fed with a centred vertical strip.

With the aim of analyzing the effect of the upper part of a monopole, the square and the circular monopoles are going to be compared with a square monopole with semi-circular base. Figure 5.38 shows the normalized current distribution for the first modes of the square monopole with semi-circular base. These modes are very similar to those depicted in Figure 5.34 for other planar monopoles.

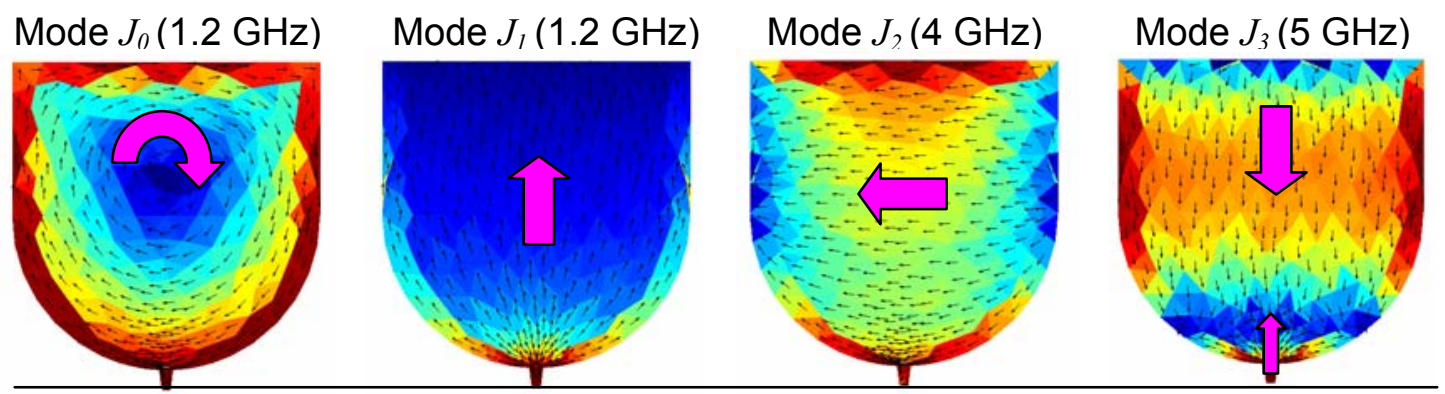

Figure 5.38 Normalized current distribution for the first modes of a square monopole with semi-circular base. 
The characteristic angle curves associated to the current modes of the square monopole with semi-circular base are illustrated in Figure 5.39. The information provided by characteristic angles regarding the resonance frequency, quality factor, and radiating bandwidth of modes is gathered in Table 5.5. It is noticeable that now mode $J_{1}$ presents wider radiating bandwidth than in the square and the circular monopole. It can also be seen that, like in the circular monopole, modes $J_{2}$ and $J_{3}$ are broad band radiators with an associated radiating bandwidth bigger than $100 \%$.

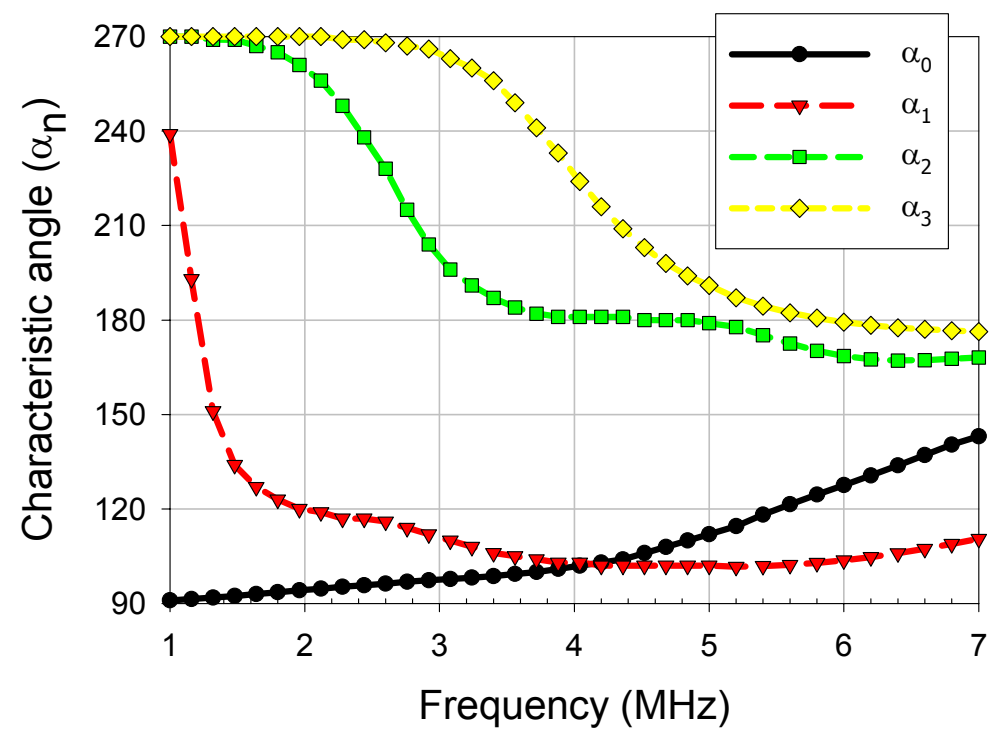

Figure 5.39 Characteristic angle variation with frequency for the current modes depicted in Figure 5.38

\begin{tabular}{|c|c|c|c|}
\cline { 2 - 4 } \multicolumn{1}{c|}{} & $f_{\text {res }}$ & $Q_{n, \text { Harrington }}$ & $B W_{n, \text { Harrington }}(\%)$ \\
\hline Mode $J_{1}$ & $1.2 \mathrm{GHz}$ & 2.95 & $33.90 \%$ \\
\hline Mode $J_{2}$ & $4.9 \mathrm{GHz}$ & 0.032 & $>100 \%$ \\
\hline Mode $J_{3}$ & $5.9 \mathrm{GHz}$ & 0.083 & $>100 \%$ \\
\hline
\end{tabular}

Table 5.5. Resonant frequencies, modal quality factors computed using (2.29), and modal radiating bandwidths for the first radiating modes of a square monopole with semicircular base.

The contribution of the VSWR of the excited modes to the total VSWR of the square monopole with semicircular base is presented in Figure 5.40. A quick inspection of these results reveals that the total VSWR of this monopole is very similar to that of the circular monopole, while it does not resemble very much the total VSWR of the square monopole. As in the circular monopole, the progressive bevelling in the base improves the matching between the excitation and the vertical mode $J_{l}$, so a broad matched band is obtained due to the contribution of modes $J_{1}$ and $J_{3}$. 


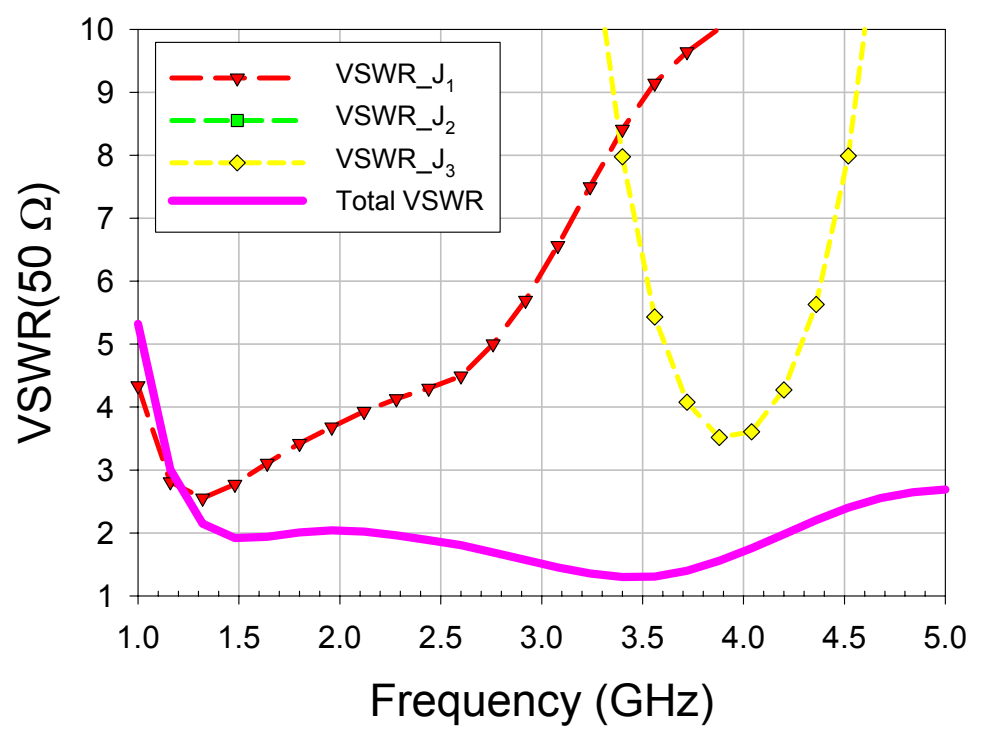

Figure 5.40 Contribution of the VSWR of modes to the total VSWR of a square monopole with semicircular base.

Hence, from these results it can be concluded that the overall performance of a square monopole with semi-circular base resembles more that of the circular monopole, than that of the square one. The explanation is that the feeding gap configuration determines the impedance bandwidth of the monopole, while the effect of the shape of its upper part over the bandwidth is not so relevant.

Note that the square monopole with semicircular base had already been reported to provide wide impedance bandwidth [130], yet no modal analysis of this monopole, previous to the one presented here, has been found in the available literature. Lastly, more examples of wideband monopoles with circular base and modified shape in the upper part can be found in [131].

\subsubsection{Modal analysis of a non-centred fed monopole.}

Now, let us examine the behaviour of a non-centred fed square monopole [132]. Figure 5.41 presents the geometry of this monopole. The size of the square monopole, and the feeding gap distance, are the same as in the square monopole analyzed in section 5.3.1.

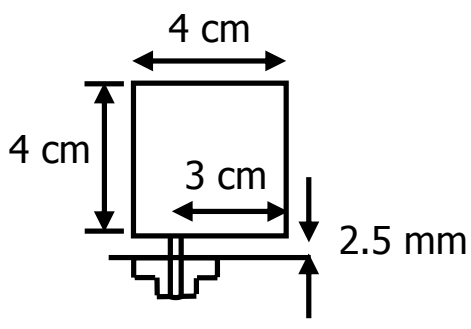

Figure 5.41 Non-centred fed square planar monopole 
Figure 5.42 shows the normalized current distribution for the first five modes of this non-symmetric square monopole. As observed, currents are distorted by the presence of an asymmetry in the structure. Special mode $J_{0}$ is not really changed, however, currents in mode $J_{l}$ are not as vertical as in the symmetric monopole, and currents in mode $J_{2}$ are not parallel to the ground plane, but flowing in diagonal direction. In mode $J_{3}$, the current null in the base of the monopole does not extend from one end to the other, but concentrates in the left part. In mode $J_{4}$, the current null in the lower left part is not exactly at the corner but a bit above, however the current distribution of the mode resembles considerably that of a symmetrical square monopole.

But, does a variation in the position of the feeding strip alter the radiating properties of modes? Figure 5.43 plots characteristic angle variation with frequency for the modes of Figure 5.42. Table 5.6 presents the resonant frequency, quality factor and radiating bandwidth of the first three radiating modes of the monopole. The fourth radiating mode $J_{4}$ has not been included, since it resonates out of the studied frequency band.

Comparing results in Table 5.6 with those obtained for the symmetrical square monopole analyzed in previous section, it can be noticed that the asymmetry created by the feeding strip has no noticeable effect on the fundamental mode $J_{l}$, while it enhances the radiating performance of the transverse current mode $J_{2}$, and degrades the efficiency of the higher order vertical current mode $J_{3}$.

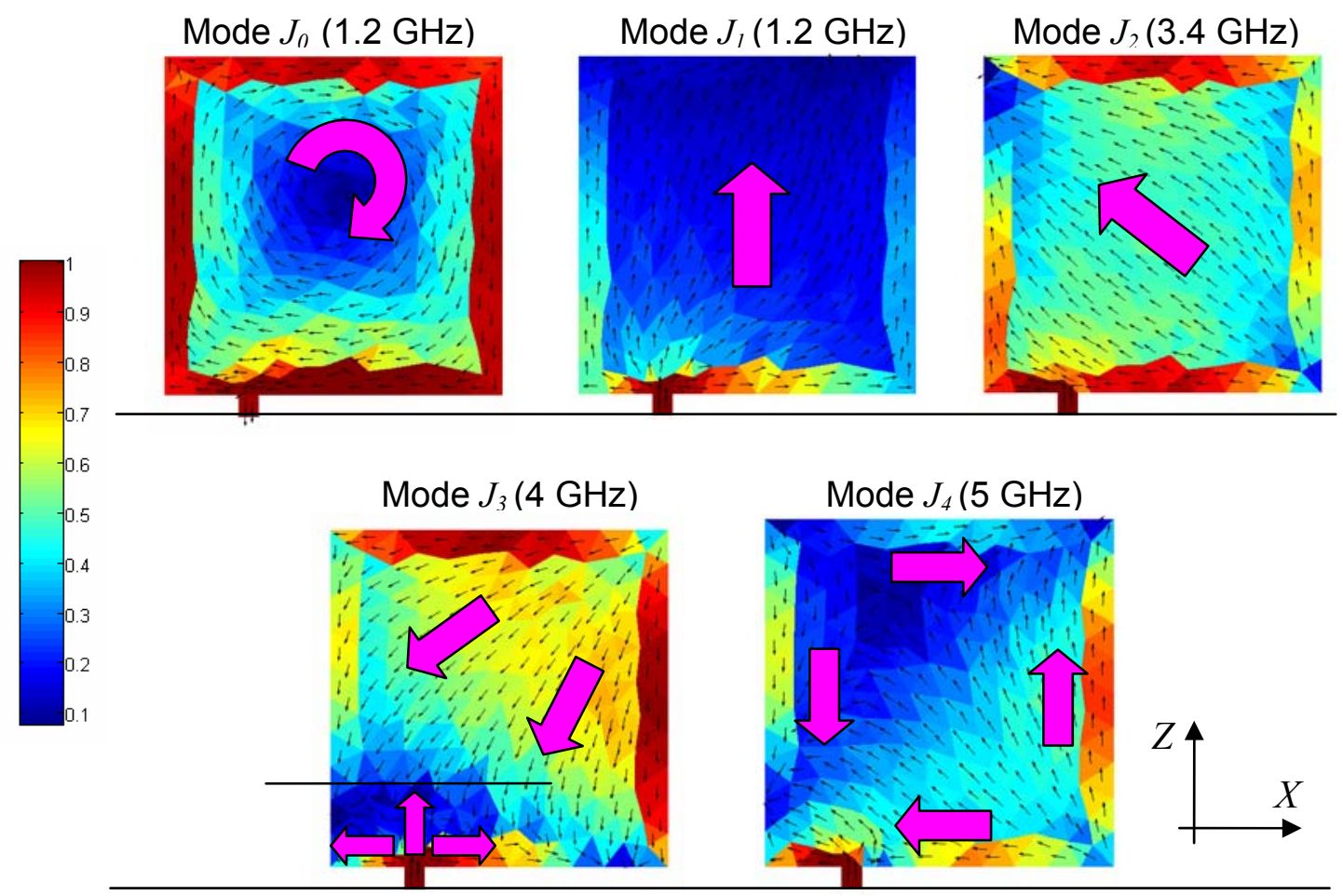

Figure 5.42 Normalized current distribution of the first five modes of a square monopole with a non-centred feeding strip. 


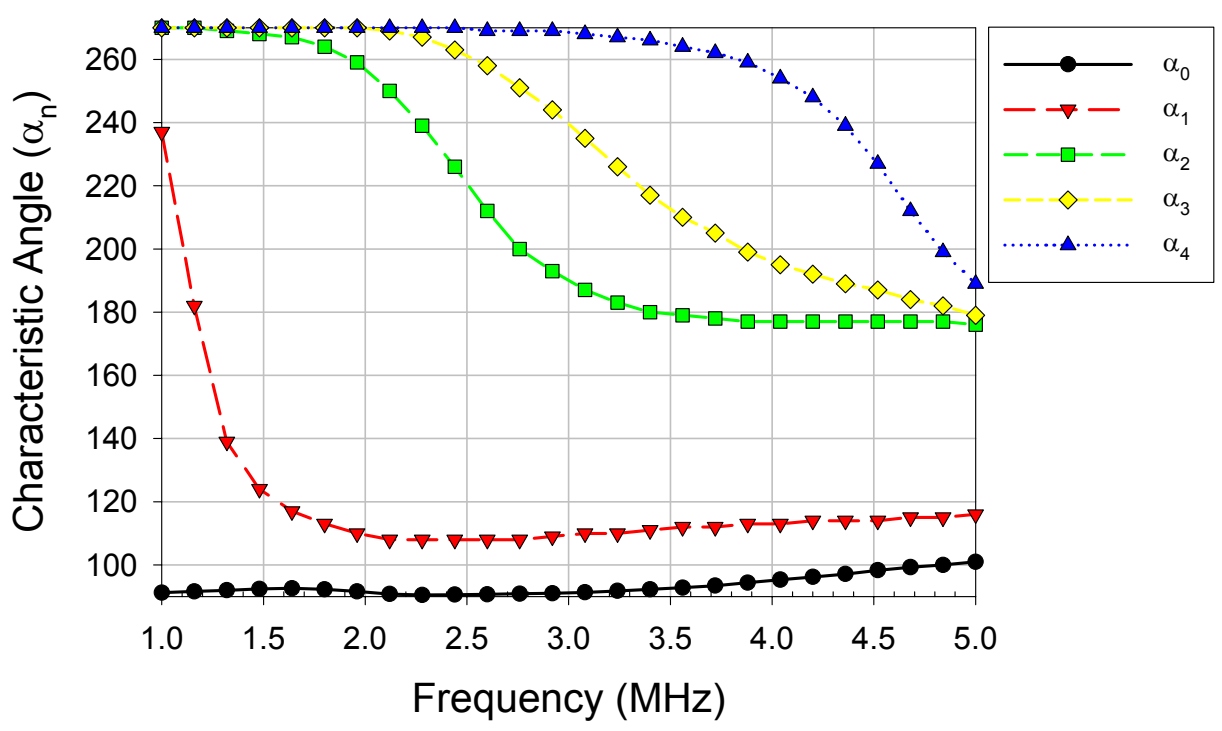

Figure 5.43 Characteristic angle variation with frequency for the current modes depicted in Figure 5.42

\begin{tabular}{|c|c|c|c|}
\cline { 2 - 4 } \multicolumn{1}{c|}{} & $f_{\text {res }}$ & $Q_{n, \text { Harrington }}$ & $B W_{n, \text { Harrington }}(\%)$ \\
\hline Mode $J_{1}$ & $1.2 \mathrm{GHz}$ & 3.35 & $29.85 \%$ \\
\hline Mode $J_{2}$ & $3.4 \mathrm{GHz}$ & 0.37 & $>100 \%$ \\
\hline Mode $J_{3}$ & $4.9 \mathrm{GHz}$ & 1.47 & $67.80 \%$ \\
\hline
\end{tabular}

Table 5.6. Resonant frequencies, modal quality factors, and modal radiating bandwidths for the first radiating modes of a non-symmetrical square monopole.

The radiation enhancement experimented by mode $J_{2}$ can be justified by the image theory. When currents of mode $J_{2}$ are pure horizontal, they are strongly cancelled by their corresponding images, especially close to the base of the monopole. For the case of the non-symmetrical monopole, mode $J_{2}$ presents diagonal currents that result in a vertical current component that is not cancelled by its image, increasing the radiating efficiency of the mode. Similarly, currents in mode $J_{3}$ are not pure vertical, so they present a horizontal component that cancels with its image, worsening the radiating behaviour of the mode. Later, it will be demonstrated that the presence of diagonal currents in the current distribution of modes $J_{1}, J_{2}$ and $J_{3}$, degrades the polarization purity of the antenna.

Nevertheless, the main difference between the symmetric and the nonsymmetric square monopole resides in the number of excited modes when a voltage generator is placed at the vertical feeding strip. As demonstrated in previous section for different planar monopole geometries, feeding configurations that preserve the symmetry of the monopole avoid the excitation of transverse and special inductive modes. 
Figure 5.44 proves that when a non-symmetric feeding is used, not only vertical current modes are excited, but also the rest of modes. Nevertheless, only modes $J_{1}, J_{3}$ and $J_{4}$ contribute to the real part of the input admittance, and hence to radiation. The rest of modes, $J_{0}$ and $J_{2}$, yet excited, do not radiate, but store energy. An examination of the imaginary part of the input admittance shows that the first resonance is due to the fundamental mode $J_{l}$. The transverse mode $J_{2}$ is weakly coupled to the feeding, and although it resonates at $3.4 \mathrm{GHz}$, this resonance does not reflect in the total susceptance, as it is hidden by the excitation of the rest of modes. The inductive behaviour of modes $J_{1}$ and $J_{0}$, compensates the capacitive behaviour of mode $J_{3}$ between $1.5 \mathrm{GHz}$ and $3.5 \mathrm{GHz}$. The result is a flat profile for the imaginary part of the input admittance in this frequency range. Similarly, the total susceptance is also flat from $3.5 \mathrm{GHz}$ to $5 \mathrm{GHz}$ as a result of the combination of the inductive modes $J_{I}$ and $J_{0}$, and the capacitive modes $J_{3}$ and $J_{4}$.
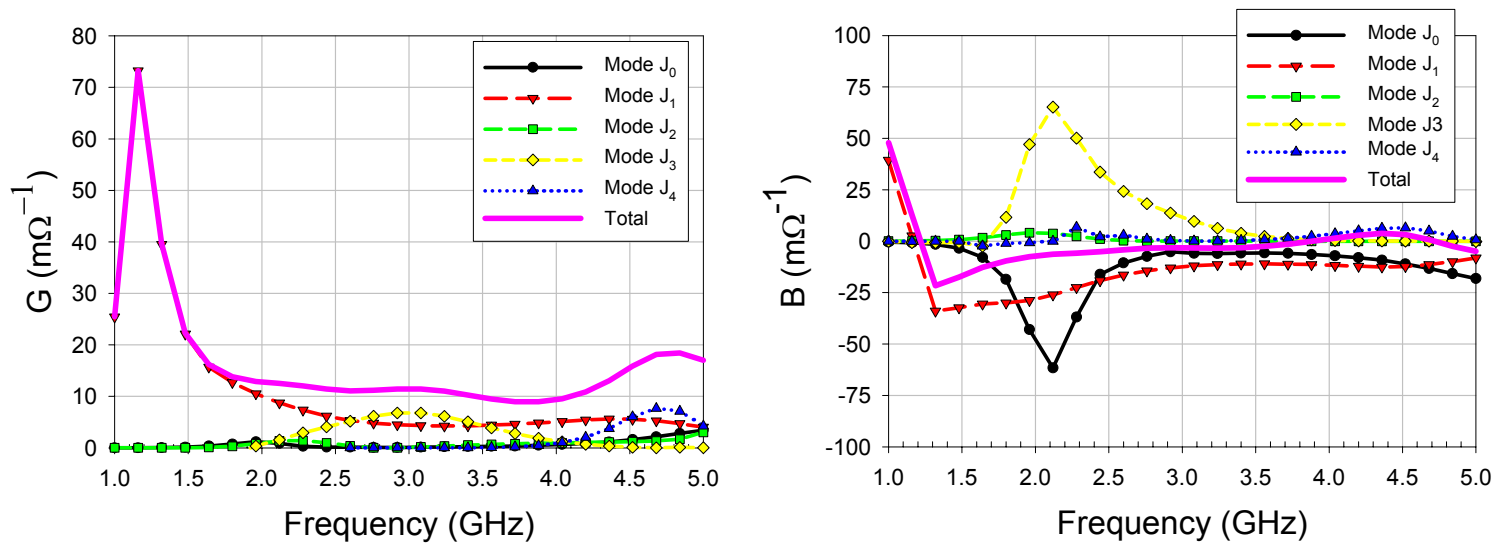

Figure 5.44 Contribution of modal admittances to the total modal admittance of the non-centred fed square monopole.

Figure 5.45 shows that only radiating modes $J_{1}, J_{3}$, and $J_{4}$, are responsible for the final VSWR of the monopole with offset feed. As observed in Figure 5.44, these three modes present similar conductance values, so the transition from one mode to the following is made in a soft way, reducing the undesirable effect of the antiresonances. Because of these soft transitions between modes the monopole presents wide impedance bandwidth. Note that a total VSWR lower than two could be easily accomplished in the whole studied frequency band, just by slightly varying the feeding gap distance, or the position of the feeding strip.

However, despite this example, in general the impedance bandwidth of an antenna does not increase with the number of excited modes. More often than not, it is difficult to achieve a soft transition between the modes of the antenna, so the impedance bandwidth does not result in that of a broadband antenna, but in that of a multi-band one. 


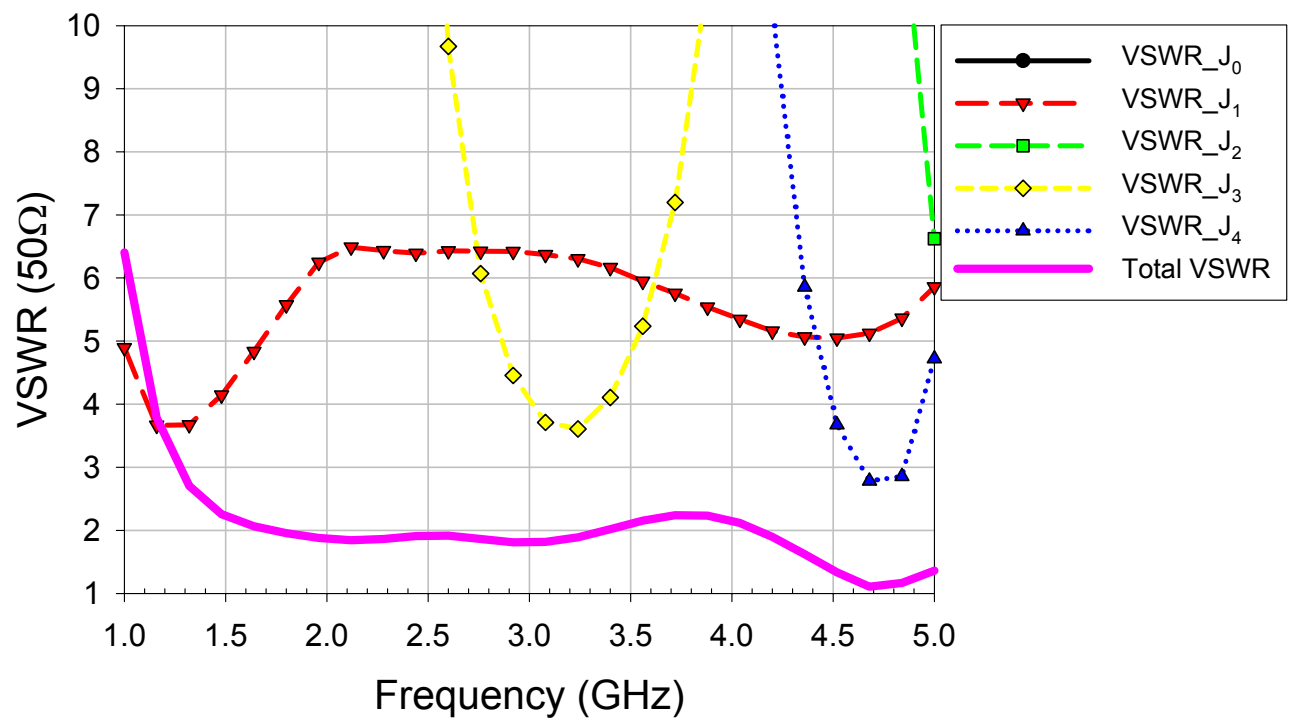

Figure 5.45 Contribution of the VSWR of modes to the total VSWR of a noncentred fed square monopole.

Let us verify now, how the polarization of the modes of the monopole is ruined by the presence of an asymmetry in the geometry. Figure 5.46 compares the elevation radiation patterns $\left(\phi=90^{\circ}\right)$ for the modal electric field produced by the first four modes of the symmetric square monopole and the non-symmetric square monopole. These modal electric fields have been computed from the modal currents, so no excitation has been considered yet. As it can be seen, all the modal radiation patterns in the $Y Z$ plane are symmetrical.

As explained before, the presence of an asymmetry in the monopole, forces the current of radiating modes to flow in diagonal direction. Thus, these modes present a horizontal and a vertical current component. It can be assumed that currents flowing in vertical direction are responsible for the $E_{\theta}$ component of the field, while currents flowing in horizontal direction generate the $E_{\phi}$ component. Hence, if polarization purity is to be achieved in planar monopoles, the excitation of horizontal currents may be avoided. The more horizontal currents are excited, the more the cross polarization level increases.

As observed in Figure 5.46 the radiation pattern of mode $J_{0}$ is very similar in both monopoles. However, the cross-polar component of the field $\left(E_{\phi}\right)$ for mode $J_{1}$, increases considerably for the non-symmetric monopole. In the symmetric monopole, the dominant component of the electric field created by mode $J_{2}$ is $E_{\theta}$, while in the non-symmetric monopole the dominant component for mode $J_{2}$ is $E_{\phi}$. With respect to the radiation pattern of mode $J_{3}$, in both monopoles the most important field component is $E_{\phi}$, while the increase in the number of radiating lobes is due to the current null at the base of the monopoles. 


\section{Symmetric square monopole}
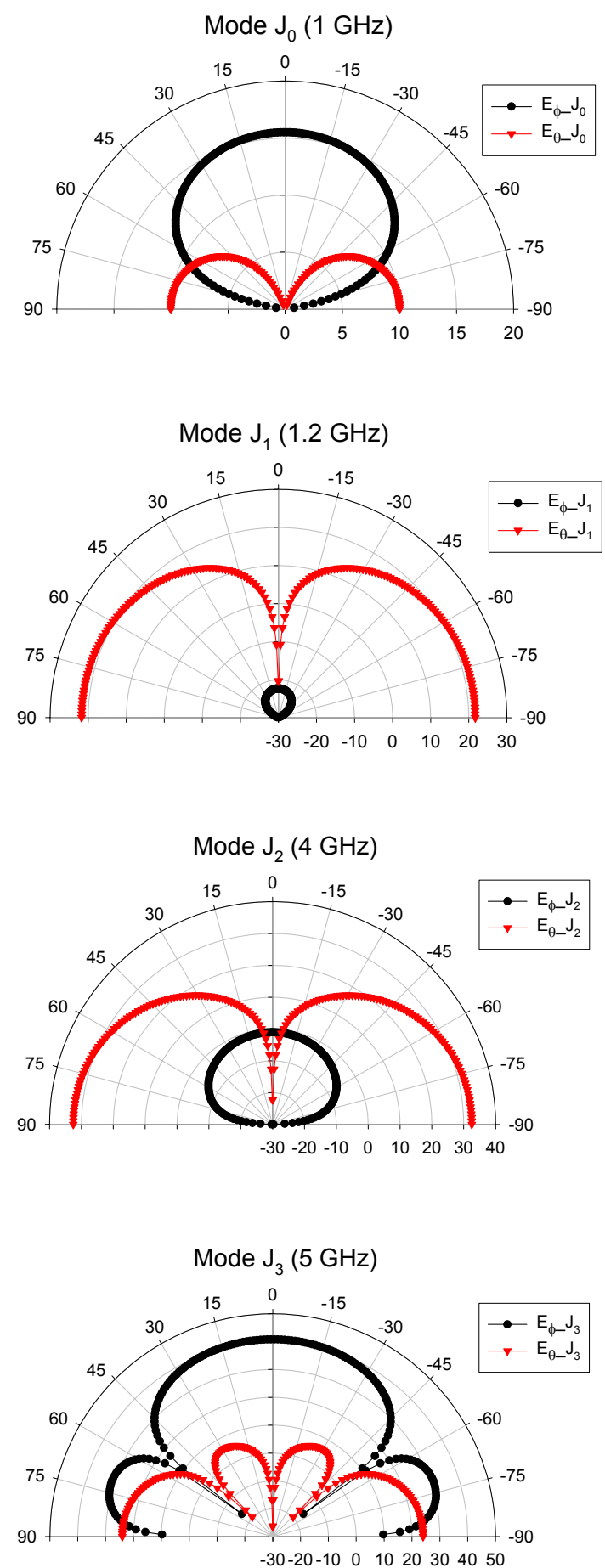

Non-symmetric square monopole

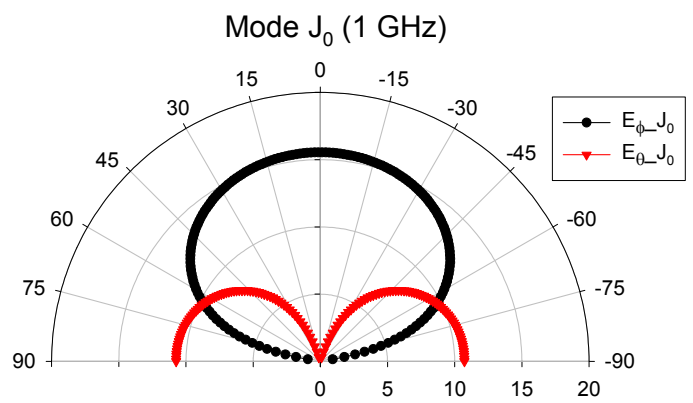

Mode $\mathrm{J}_{1}(1.2 \mathrm{GHz})$

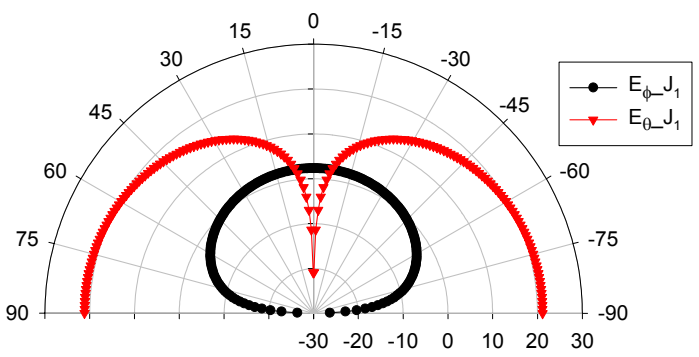

Mode $\mathrm{J}_{2}(4 \mathrm{GHz})$

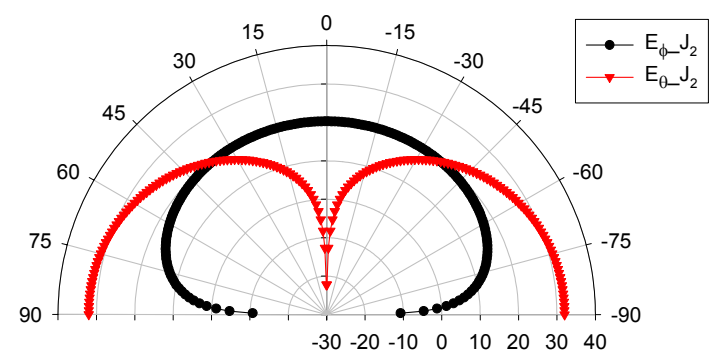

Mode $\mathrm{J}_{3}(5 \mathrm{GHz})$

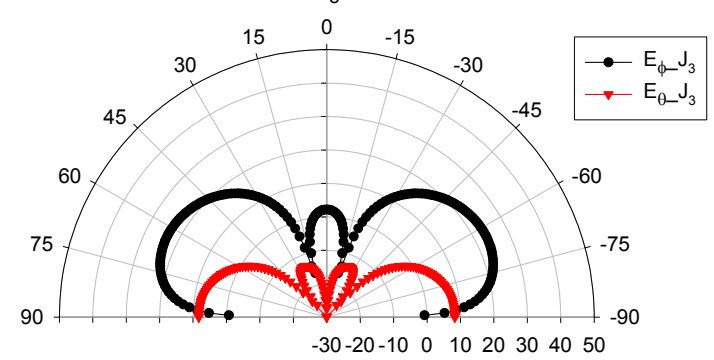

Figure 5.46 Elevation radiation patterns $\left(\phi=90^{\circ}\right)$ of the modal electric field produced by the first four modes of the centre-fed square monopole and the non-centred fed square monopole 
To validate the polarization degradation experimented by the non-centred fed square monopole when excited at the vertical feeding strip, the total current for both the centre fed and the non-centred fed monopole, as well as its associated radiation patterns, are going to be compared.

Figure 5.47 shows the real and imaginary parts of the total current for the centred fed square monopole at $1.2 \mathrm{GHz}$ and $4 \mathrm{GHz}$. Figure 5.48 presents the same results for the non-centred fed square monopole. Let us begin analyzing the centre fed monopole. Both the real and imaginary parts of the total current at $1.2 \mathrm{GHz}$ correspond with the current distribution of the fundamental mode $J_{1}$. The same happens with the components of the total current at $4 \mathrm{GHz}$, which exhibit the current distribution of the higher order vertical current mode $J_{3}$. Nevertheless, the behaviour of the components of the total current for the noncentred fed monopole is a bit different, since the real part is due to the excitation of one mode, while the imaginary part is due to the excitation of a different one. Observe that in Figure 5.48, the current distribution of the real part is that of the fundamental mode $J_{1}$ at $1.2 \mathrm{GHz}$, and that of mode $J_{3}$ at 4 $\mathrm{GHz}$. Conversely, the imaginary part of the total current is caused by the excitation of transverse mode $J_{2}$ at $1.2 \mathrm{GHz}$, and by the excitation of mode $J_{4}$, which also presents a transverse component, at $4 \mathrm{GHz}$.

Lastly, Figure 5.49 compares the elevation radiation patterns at two different planes, generated by the total currents considered above. As expected the cross polarization level is higher for the non-centred fed monopole than for the centred feed one. Moreover, the effect of the asymmetry introduced by the feeding can be observed at the $X Z$ plane at $4 \mathrm{GHz}$.

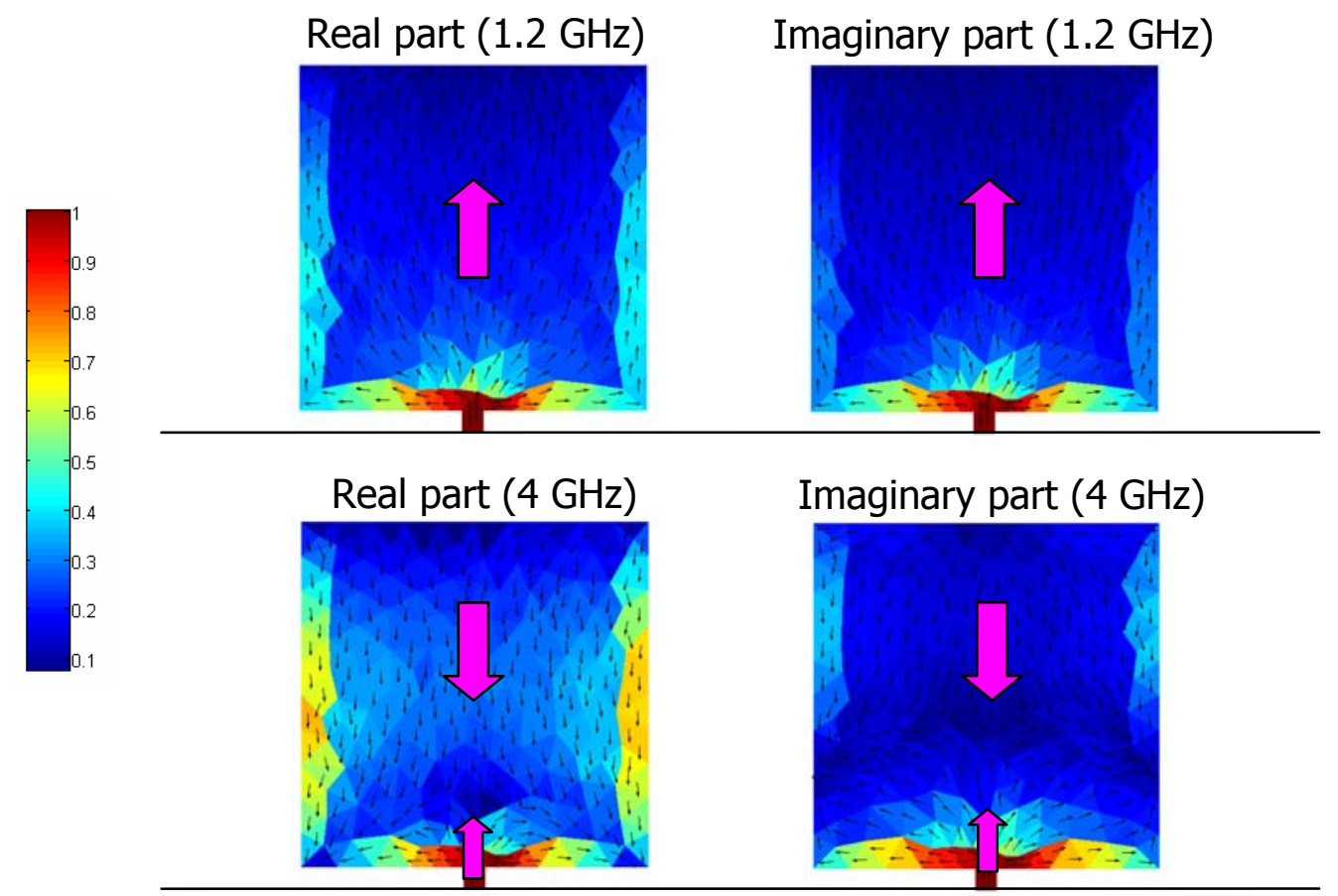

Figure 5.47 Real and imaginary parts of the total current for a centred fed square monopole at $1.2 \mathrm{GHz}$ and $4 \mathrm{GHz}$. 


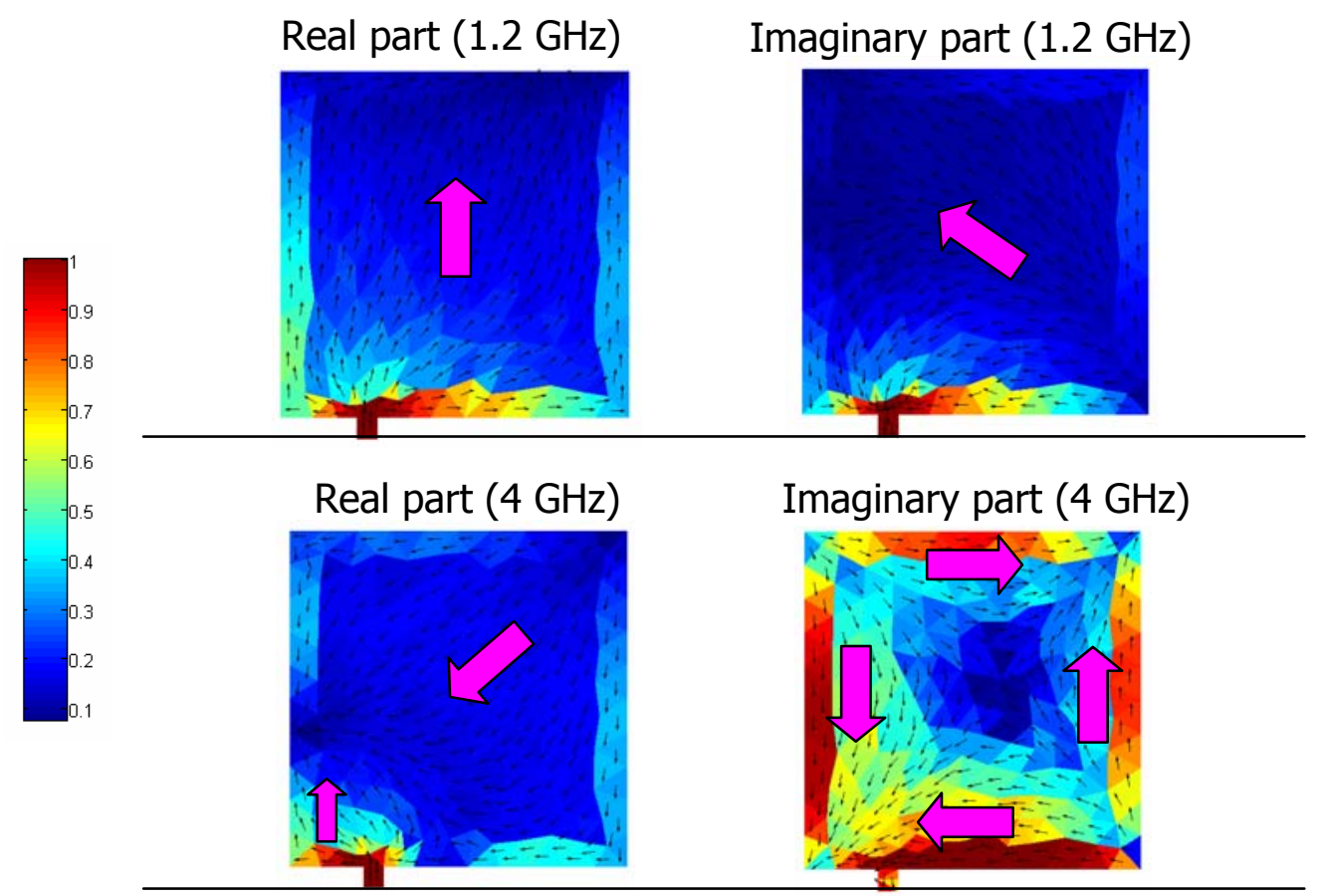

Figure 5.48 Real and imaginary parts of the total current for a non-centred fed square monopole at $1.2 \mathrm{GHz}$ and $4 \mathrm{GHz}$.

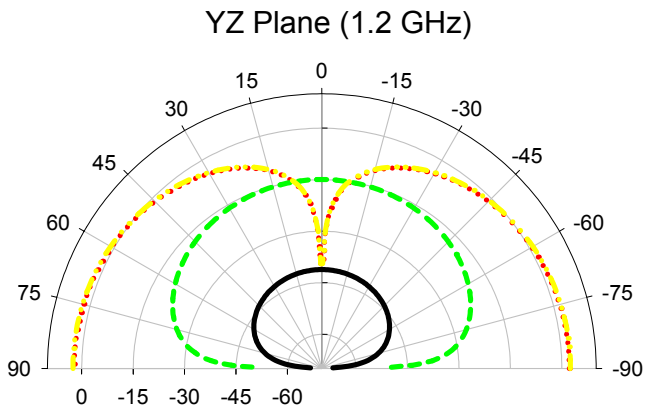

YZ Plane $(4 \mathrm{GHz})$

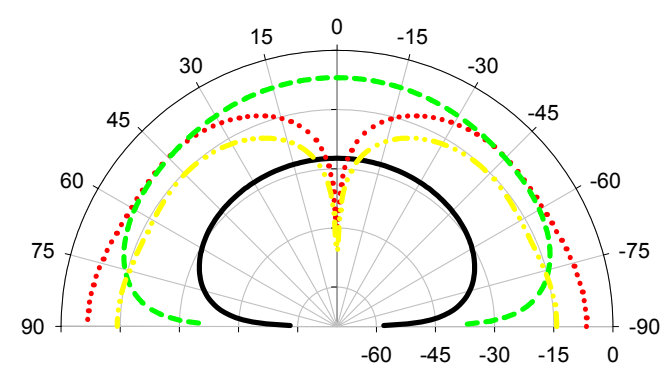

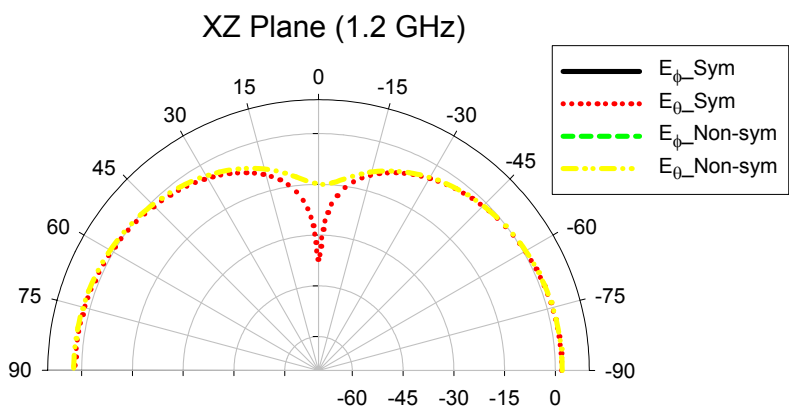

XZ Plane $(4 \mathrm{GHz})$

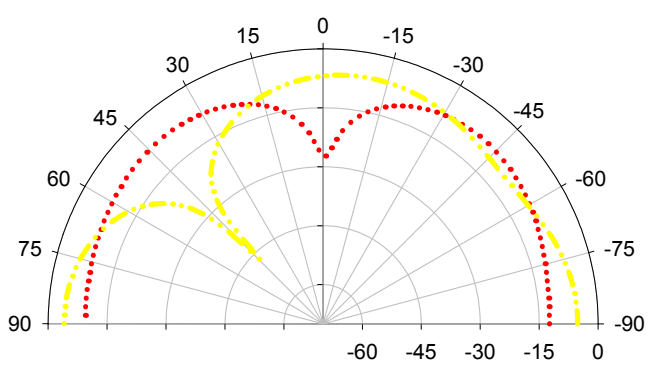

Figure 5.49 Radiation patterns at $1.2 \mathrm{GHz}$ and $4 \mathrm{GHz}$ for the centred fed square monopole and the non-centred fed square monopole.

Note that for the brevity, the azimuthal radiation patterns due to the total currents have not been included since all of them are omnidirectional, and they do not supply important information. 


\subsubsection{Effect of the addition of a shorting pin on the monopole base.}

Another well-known technique to increase the impedance bandwidth of planar monopoles consists in adding a shorting pin to one of the ends on the base of the monopole [125]-[127]. The insertion of this shorting pin creates an asymmetry on the monopole that allows the excitation of transverse and special inductive modes.

But, has the asymmetry created by the shorting pin the same effect over the impedance bandwidth than the asymmetry due to a non-centred feeding strip? Which solution is more respectful with the polarization of the monopole? The purpose of this section is to answer these questions with the information yield by characteristic modes. Figure 5.50 shows the geometry of the shorted square monopole that is going to be analyzed as example. Its dimensions are identical to those of the square monopoles already studied in previous sections.

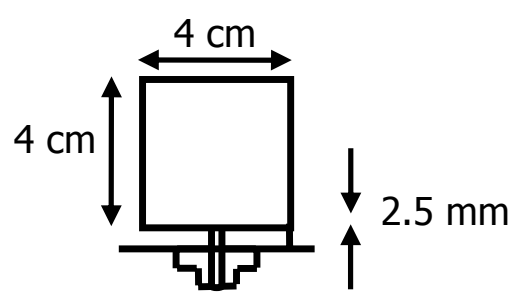

Figure 5.50 Square monopole with a shorting pin.

As usual, to begin the study, Figure 5.51 presents the normalized current distribution for the first modes of the shorted square monopole. The current of these modes behaves more or less like in other planar monopoles. There is a special inductive mode $\left(J_{0}\right)$, a vertical current mode $\left(J_{1}\right)$, a horizontal current mode $\left(J_{2}\right)$, a higher order vertical current mode $\left(J_{3}\right)$, and a mode with both vertical and horizontal currents $\left(J_{4}\right)$. In all modes, the current flowing in the feeding strip is very intense, so all this modes are prone to be excited by a voltage generator placed at this vertical strip. Likewise, currents in the vertical shorting pin are also intense. As observed, the asymmetry introduced by the pin shifts the position of current nulls with respect to its position in a common symmetrical non-shorted square monopole.

Figure 5.52 depicts the characteristic angle curves for these modes. Table 5.7 comprises the information for the resonant frequency, quality factor and radiating bandwidth of modes. The conclusions that can be extracted from these results are similar to the same ones that were obtained for the non-centred fed square monopole. The effect of the asymmetry does not affect very much modes $J_{1}$ and $J_{3}$, whereas it enhances the radiating bandwidth of mode $J_{2}$. Once more, these variations in the efficiency of modes can be attributed to the presence of diagonal currents. Like in the non-centred fed monopole, the presence of modal currents flowing in diagonal direction may increase the level of the cross-polar component of the electric field. 


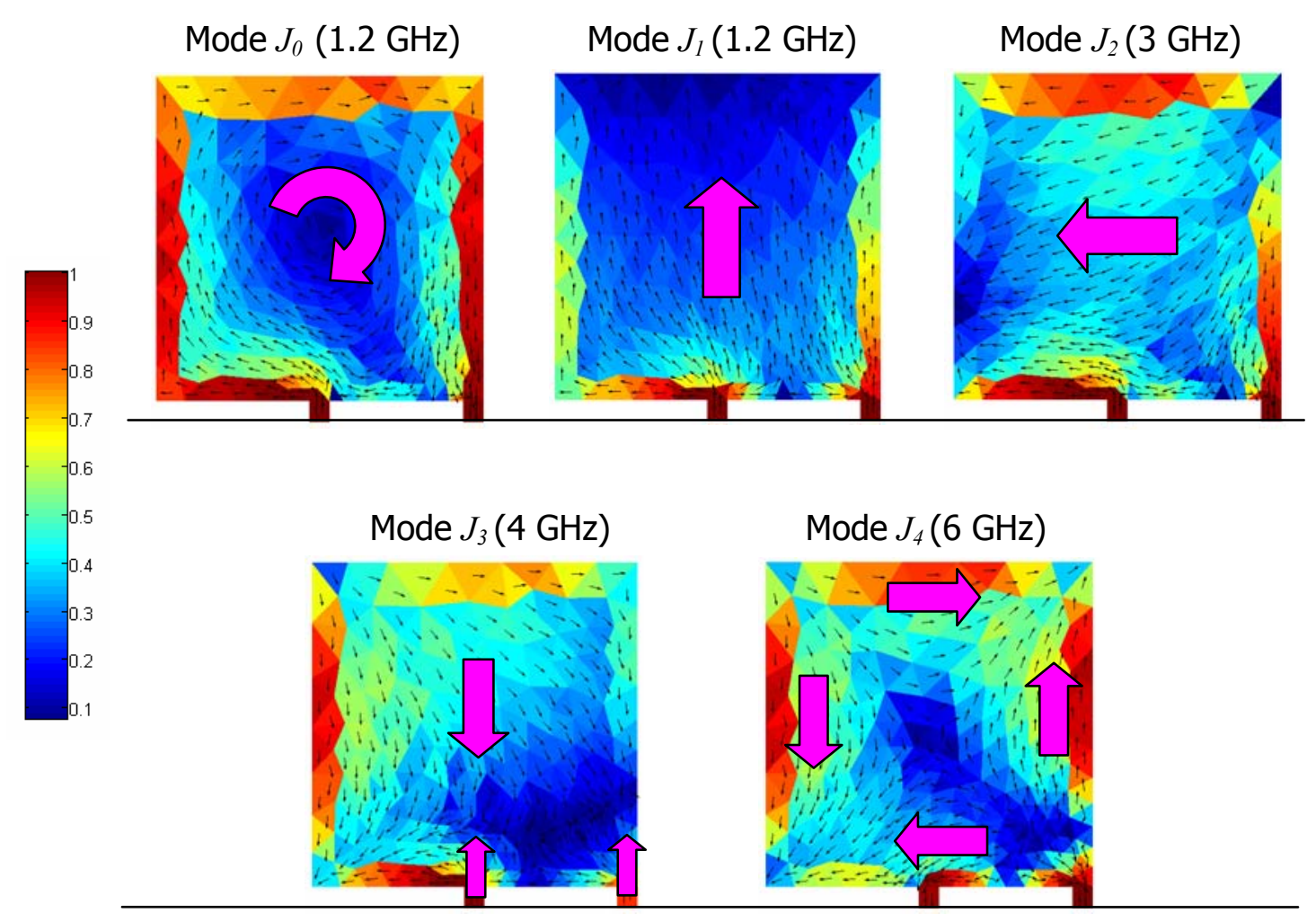

Figure 5.51 Normalized current distribution for the first modes of the shorted square monopole.

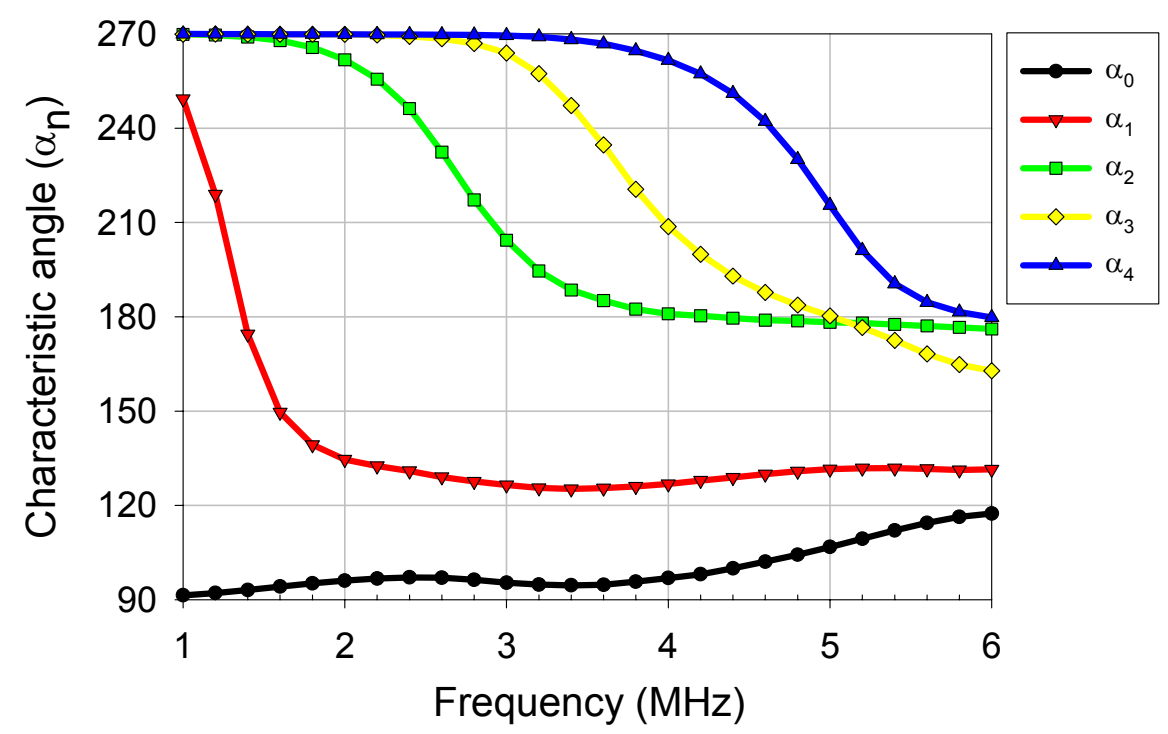

Figure 5.52 Characteristic angle variation with frequency for the firs modes of a shorted square monopole. 


\begin{tabular}{|c|c|c|c|}
\cline { 2 - 4 } \multicolumn{1}{c|}{} & $f_{\text {res }}$ & $Q_{n, \text { Harrington }}$ & $B W_{n, \text { Harrington }}(\%)$ \\
\hline Mode $J_{1}$ & $1.3 \mathrm{GHz}$ & 2.94 & $34 \%$ \\
\hline Mode $J_{2}$ & $4.3 \mathrm{GHz}$ & 0.15 & $>100 \%$ \\
\hline Mode $J_{3}$ & $5.1 \mathrm{GHz}$ & 0.82 & $>100 \%$ \\
\hline Mode $J_{4}$ & $5.9 \mathrm{GHz}$ & 0.43 & $>100 \%$ \\
\hline
\end{tabular}

Table 5.5. Resonant frequencies, modal quality factors computed using (2.29), and modal radiating bandwidths for the first radiating modes of a shorted square monopole.

However, which modes contribute to the radiation of the monopole when it is excited at the vertical strip? Figure 5.53 sketches how the modal admittances contribute to the total admittance of the shorted square monopole. Modes $J_{1}$ and $J_{3}$ are the most relevant modes for the real part of the admittance. Nevertheless, the rest of modes are also excited, and they also help to give shape to the real part of the total input admittance. The real part of the admittance of mode $J_{2}$ takes significant values just before its resonance, from 2 $\mathrm{GHz}$ to $4 \mathrm{GHz}$. Surprisingly, the special mode $J_{0}$ also contributes considerably to radiation from $2 \mathrm{GHz}$ to $4 \mathrm{GHz}$. In Figure 5.51 it can be appreciated that not all currents in mode $J_{0}$ form closed loops. The more intense currents of this mode flow uninterrupted from the vertical feeding strip to the shorting pin, and probably these currents are the reason for the increase of radiation. Finally, mode $J_{4}$, yet excited, only contributes slightly to the total conductance from 5 $\mathrm{GHz}$ to $6 \mathrm{GHz}$.

On the other hand, the imaginary part of the total admittance results from the combination of the modal admittances of all the five modes. The only identifiable resonance happens at $1.3 \mathrm{GHz}$ and it is forced by mode $J_{l}$. At the rest of frequencies, the imaginary part of the total admittance keeps quite a flat profile, as a consequence of the compensation between capacitive and inductive modes.

If the admittances of Figure 5.53 are compared with the admittances of Figure 5.44, it arises that for the case of the non-centred fed square monopole, the excitation of modes $J_{0}$ and $J_{2}$ was only perceptible at the imaginary part of the admittance. By contrast, for the case of the shorted square monopole the excitation of modes $J_{0}$ and $J_{2}$ is reflected in both the real and imaginary parts of the input admittance.

Figure 5.54 reveals that the final shape of the total $V S W R$ is basically determined by the modal VSWR of mode $J_{1}$. Mode $J_{3}$ helps to improve the total matching from $3.4 \mathrm{GHz}$ to $4 \mathrm{GHz}$. Modes $J_{2}$ and $J_{0}$ and are not very well matched, but they aid to improve the matching of the total $V S W R$, from $2.5 \mathrm{GHz}$ to $3 \mathrm{GHz}$, for the case of mode $J_{2}$, and for frequencies higher than $5.5 \mathrm{GHz}$ for mode $J_{0}$. 

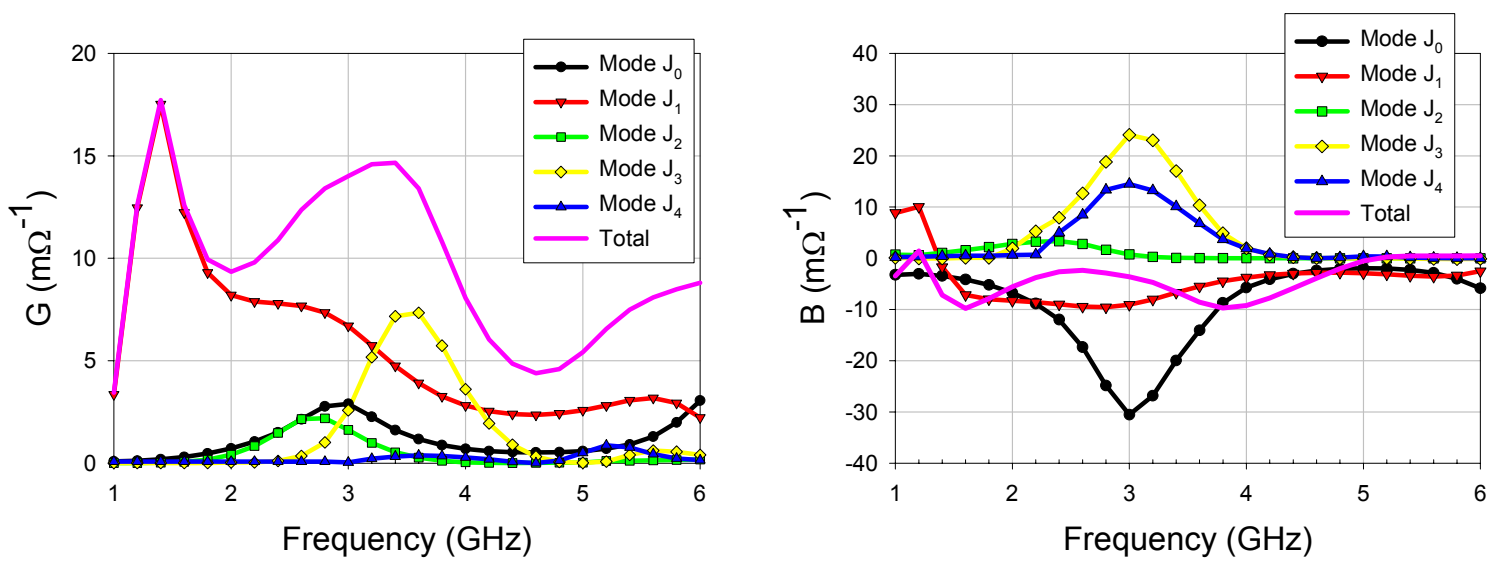

Figure 5.53 Contribution of modal admittances $Y_{n}$ to the total input admittance $Y_{\text {in }}$ of different planar monopole geometries.

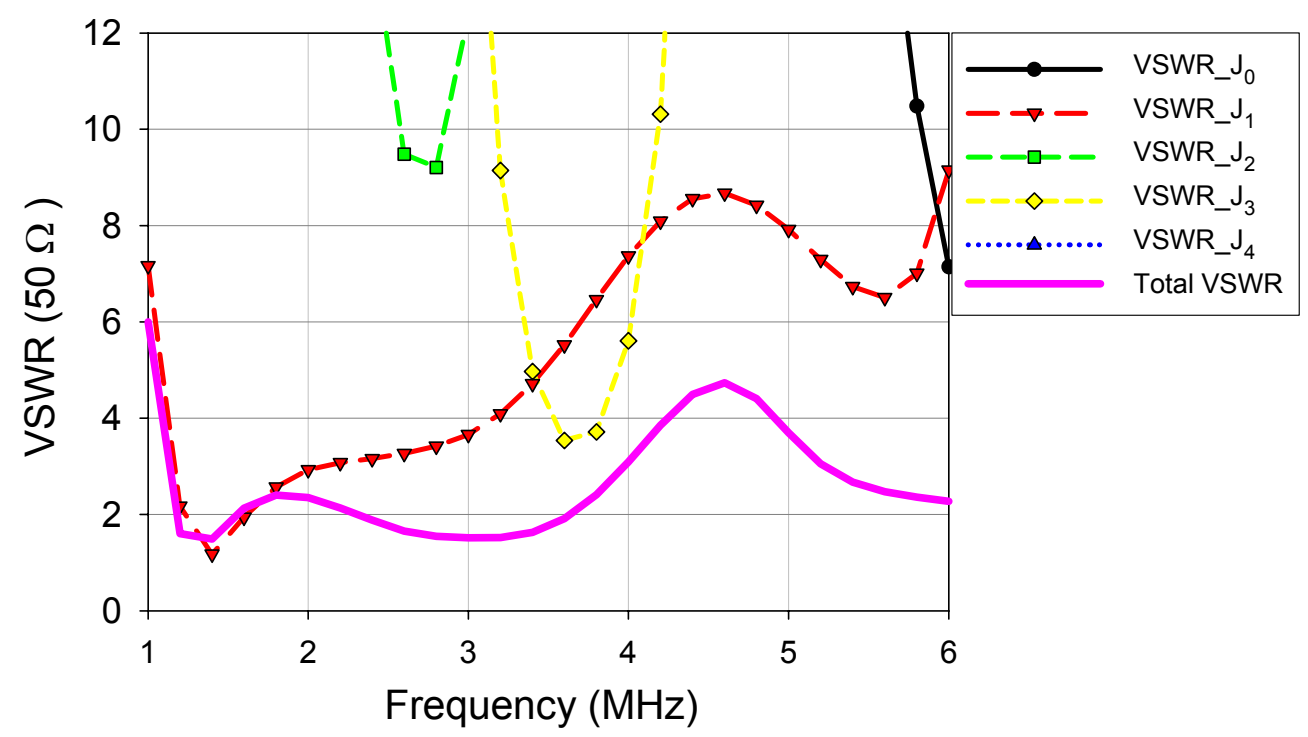

Figure 5.54 Contribution of the VSWR of modes to the total VSWR of a the shorted square monopole.

But now it raises the question, does the shorted square monopole present lower cross-polarization levels than the non-centred fed monopole? Figure 5.55 depicts the real and imaginary parts of the total current for the shorted square monopole at $1.2 \mathrm{GHz}$ and $4 \mathrm{GHz}$. At first glance, it seems that the flow of transverse currents is lower in this monopole than in the non-centred fed one. However, these results are not conclusive.

To verify that the shorted square monopole presents lower cross polarization levels than the non-centre fed square monopole, the elevation radiation patterns generated by the total electric field of these two monopoles are going to be analyzed at $1.2 \mathrm{GHz}$ and $4 \mathrm{GHz}$. 


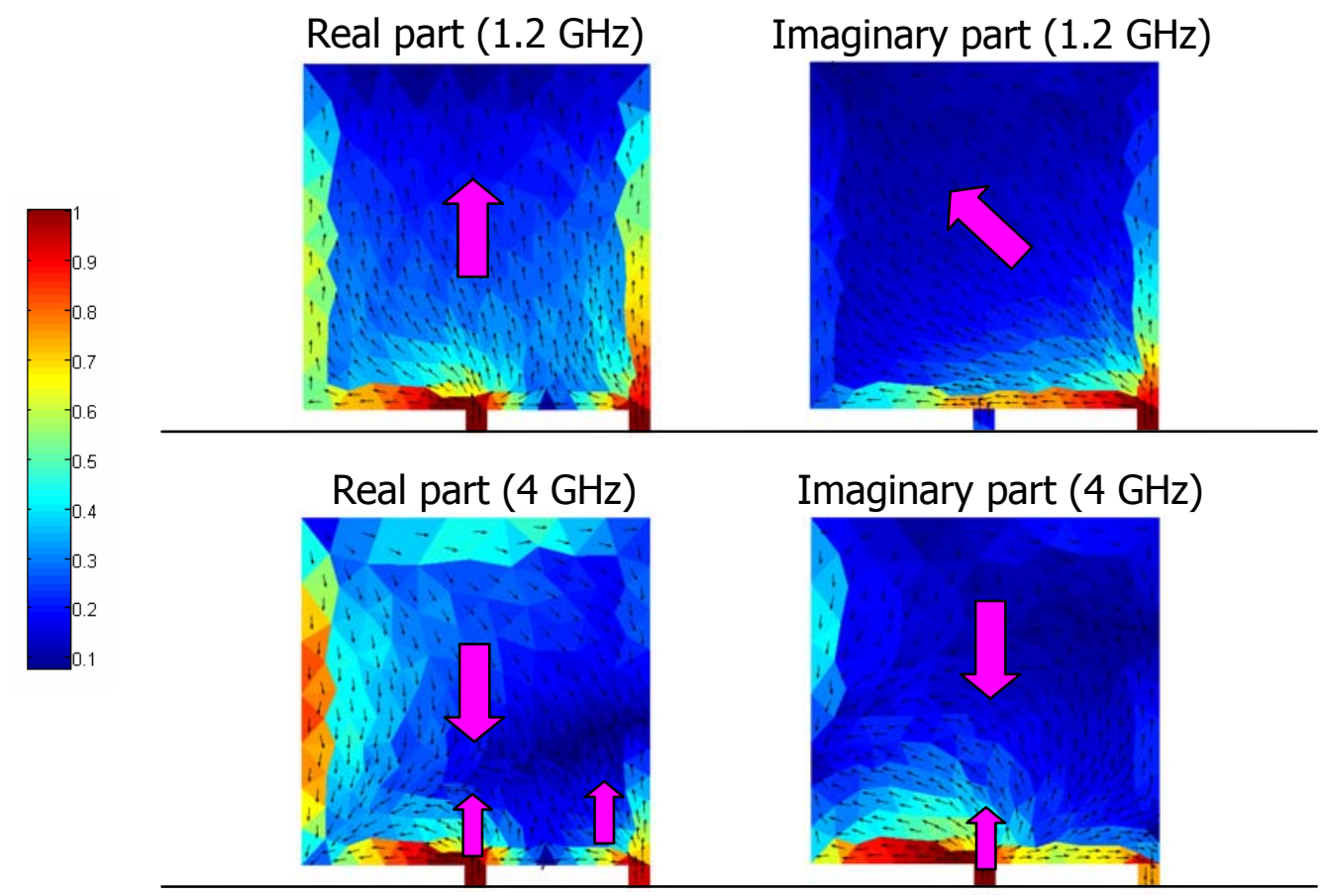

Figure 5.55 Real and imaginary parts of the total current for a non-centred fed square monopole at $1.2 \mathrm{GHz}$ and $4 \mathrm{GHz}$.

Figure 5.56 compares the above mentioned radiation patterns. As expected, the $E_{\phi}$ component of the field in the $Y Z$ plane is smaller for the shorted square than for the non-centred fed square at both $1.2 \mathrm{GHz}$ and $4 \mathrm{GHz}$, although there is no much difference between them. However, it should be remarked that the asymmetry created by the shorting pin is not as noticeable in the $X Z$ plane as it is the asymmetry due to the non-centred feeding, in special at highest frequencies. Observe that at $4 \mathrm{GHz}$, the shorted square monopole does not present the radiation null at $45^{\circ}$ exhibited by the non-centred fed monopole.

For the sake of comparison, Figure 5.57 shows the total VSWR computed for all the square monopoles already analyzed. As observed, the non-centred fed square monopole presents the widest impedance bandwidth for $V S W R<2.5$. Nevertheless, this monopole is the one which exhibits the highest cross polarization level, as well as radiating nulls, due to the non-centred feeding. Just the opposite, the symmetric square monopole presents the narrowest matched band for $V S W R<2.5$, but the purest polarization, and the most symmetric radiation patterns. That is why this monopole may be the perfect choice when the polarization purity is important.

Hence, it seems that the shorted square monopole is an intermediate solution, as it presents a trade off between impedance bandwidth and radiation pattern bandwidth. Moreover, this monopole yields the lowest matched frequency, so it constitutes the most compact solution. 

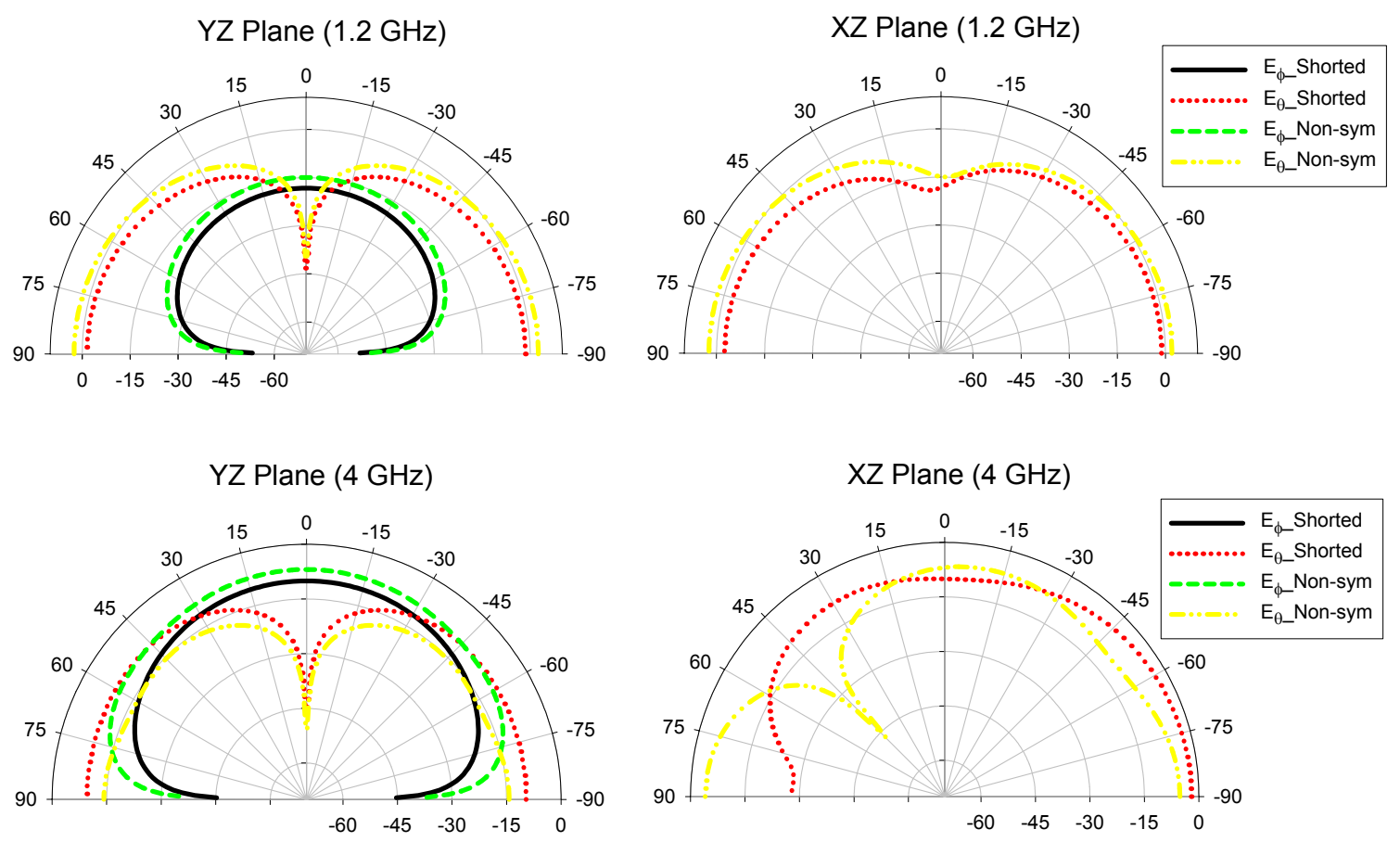

Figure 5.56 Radiation patterns at $1.2 \mathrm{GHz}$ and $4 \mathrm{GHz}$ for the centred fed square monopole and the non-centred fed square monopole.

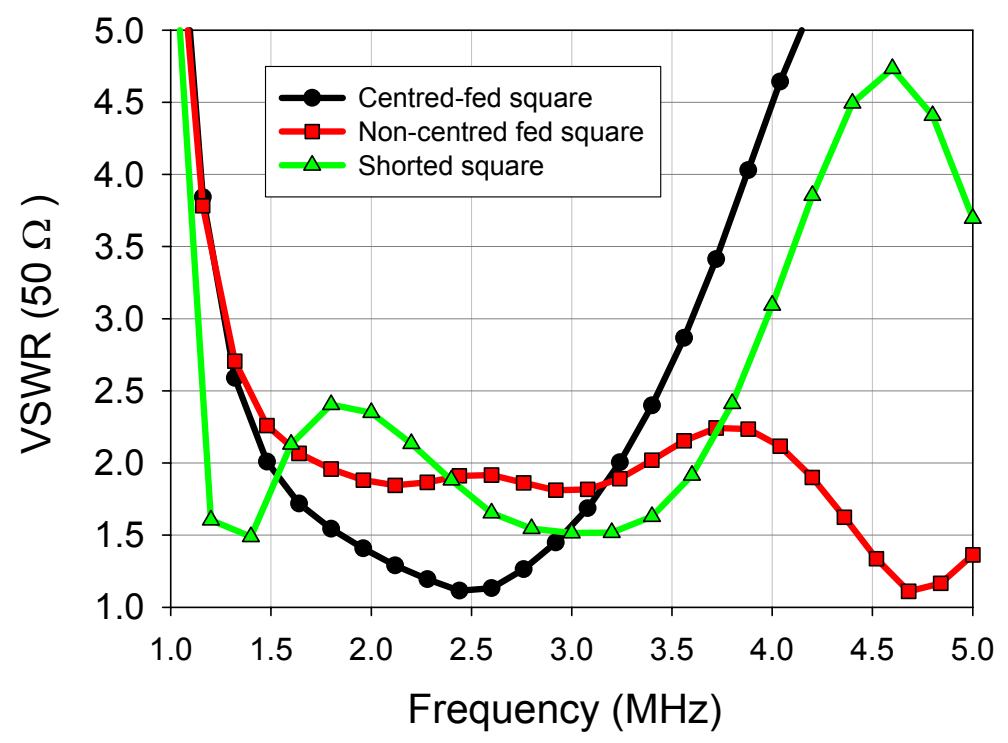

Figure 5.57 Comparison of the total VSWR for the centred-fed square monopole, the non-centred fed square monopole and the shorted square monopole.

Summing up, although the impedance bandwidth of the shorted square monopole is not as wide as the bandwidth of the non-centred fed monopole, sometimes this monopole may be preferred, since its radiation patterns are reasonably symmetric, and its lowest matched frequency is smaller than that provided by the non-centred fed monopole. 
As explained in section 5.3.1 monopoles with a proper bevelled base, provide wider impedance bandwidth than its non-bevelled counterparts. Thus, if the impedance bandwidth yielded by the shorted square monopole were not wide enough, the base of the monopole could be properly bevelled in order to increase the matched band [133].

Since the square monopole has already been thoroughly examined, let us validate this last statement using a reverse bow-tie monopole. As commented in section 5.3.1, the behaviour of a square monopole and a reverse bowtie monopole is very similar, as they both present the same non-bevelled feeding configuration.

Figure 5.58 shows the different monopoles derived from the reverse bow-tie monopole that are going to be studied: (a) Reverse bowtie monopole, (b) Shorted reverse bowtie monopole, (c) Bevelled reverse bowtie monopole, henceforth called arrowhead monopole, and (d) Shorted arrowhead monopole. All the monopoles are placed over an infinite perfect ground plane, and are fed at the middle of its base. In all cases, the height is $H=4 \mathrm{~cm}$, and the feeding gap distance is $h=2.5 \mathrm{~mm}$. For the arrowhead monopole the distance $h_{2}$ is 8.5 $\mathrm{mm}$. Figure 5.59 compares the VSWR obtained for these monopoles using the electromagnetic simulator WIPL-D. As observed, because of the bevelling, the arrowhead presents wider bandwidth than the reverse bow-tie. Moreover, the shorted versions of both these two monopoles provide broader matched bands than the non-shorted ones.

Thus, the shorted arrowhead provides the widest impedance bandwidth due to the combination a shorting pin with a bevelled base. Yet, an even broader impedance bandwidth could be achieved, if the dimensions of this shorted arrowhead monopole were correctly optimized.

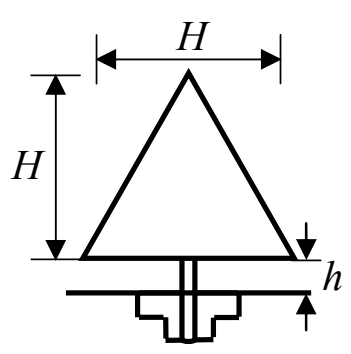

(a)

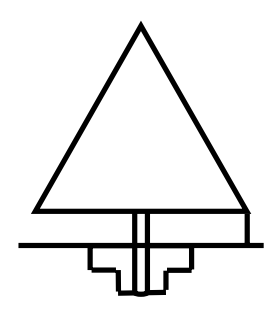

(b)

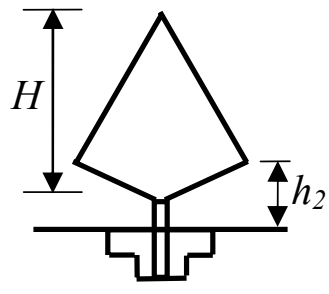

(c)

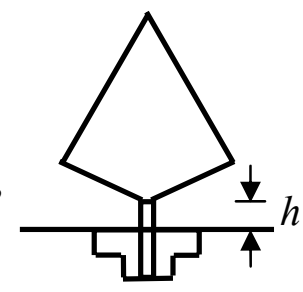

(d)

Figure 5.58 Different planar monopoles derived from a reverse bowtie monopole: (a) Reverse bowtie monopole, (b) Shorted reverse bowtie monopole,

(c) Arrowhead monopole, and (d) Shorted arrowhead monopole. 


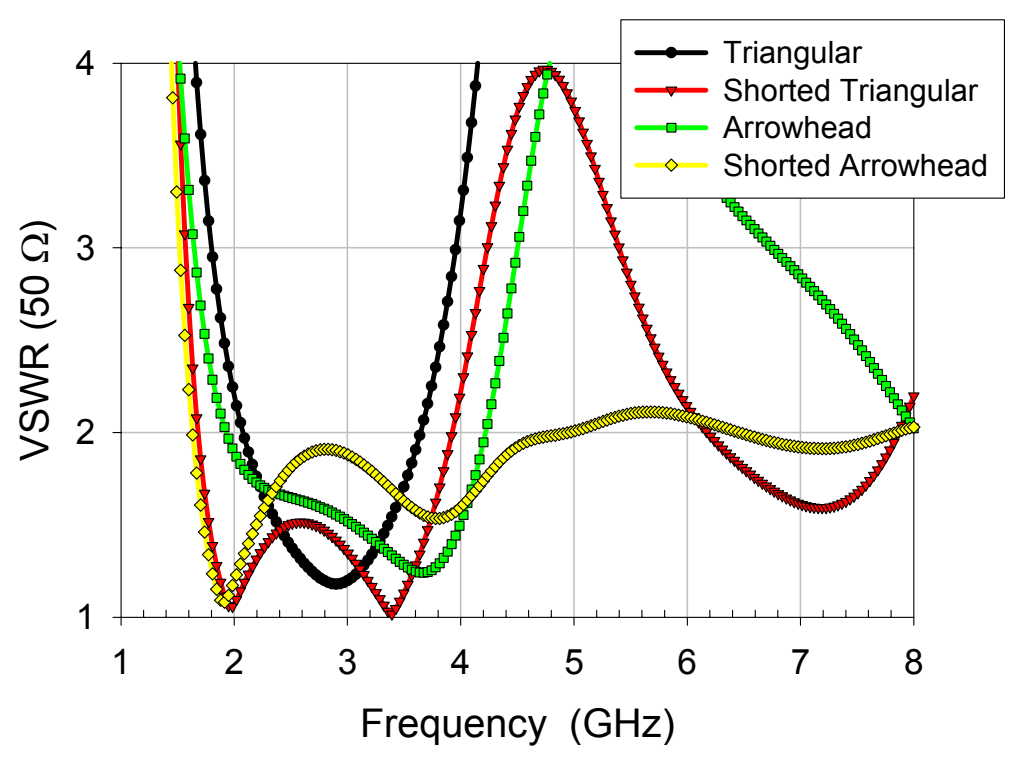

Figure 5.59 VSWR referred to $50 \Omega$ computed for the monopoles of Figure 5.58 using WIPL-D.

After using a genetic algorithm in combination with WIPL-D, the optimum dimensions of the shorted arrowhead monopole, to obtain the widest impedance bandwidth have been found to be: $H=46 \mathrm{~mm}, h=1 \mathrm{~mm}$, and $h_{2}=7 \mathrm{~mm}$. The final design, published in [134], is sketched in Figure 5.60 (a). Note the dimensions of the monopole may be scaled to reduce the low frequency of the matched band if necessary.

Figure 5.60 (b) shows a photograph of a prototype of the antenna fabricated at the laboratories of the GRE (Electromagnetic Radiation Group) in UPV (Technical University of Valencia). This prototype includes a $20 \mathrm{~cm}$ square aluminium ground plane, and it is fed via a standard SMA connector.

Figure 5.61 compares the VSWR measured for the prototype with the VSWR simulated with different numerical codes: WIPL-D, IE3D and our own code based on the method of moments. As observed, IE3D is the code that predicts in a more close way the values of the measured $V S W R$, which are a bit higher than 2 from $3.8 \mathrm{GHz}$ to $4.5 \mathrm{GHz}$. This small mismatch could be avoided just by modifying slightly the feed gap separation $h$, which has proved to be a very critical parameter.

Results obtained with the code based on the method of moments are also in close agreement with measured results. In contrast, the most imprecise simulator has been discovered to be WIPL-D. For the case of WIPL-D, results are very sensitive to the feeding gap distance $h$, so the curve obtained for the $V S W R$ is a bit shifted in frequency. Anyway, the impedance bandwidth measured for the shorted arrowhead monopole is larger than $100 \%$ for $V S W R<2.1$. 


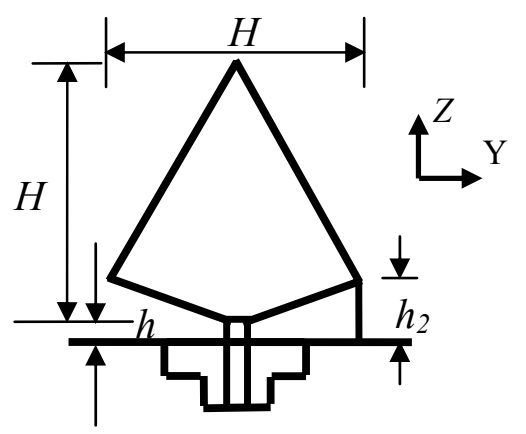

(a)

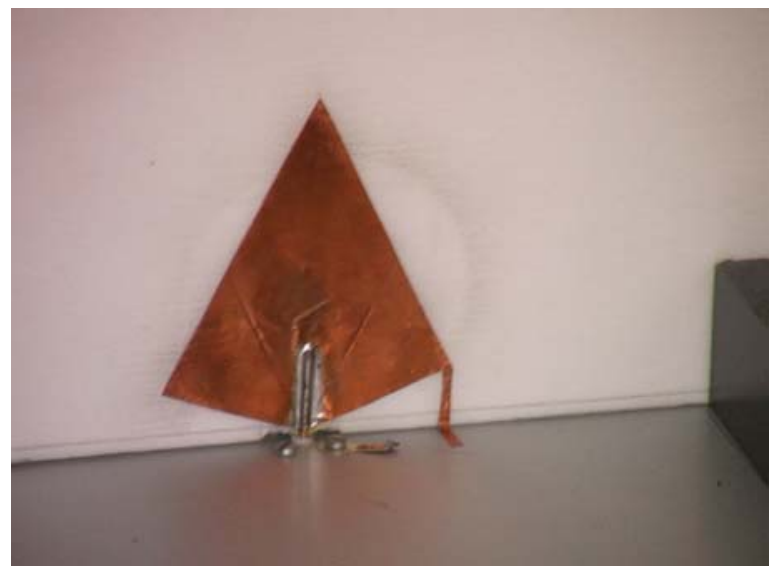

(b)

Figure 5.60 (a) Optimum dimensions obtained for the shorted arrow tip monopole. (b) Photograph of a prototype of the optimized antenna.

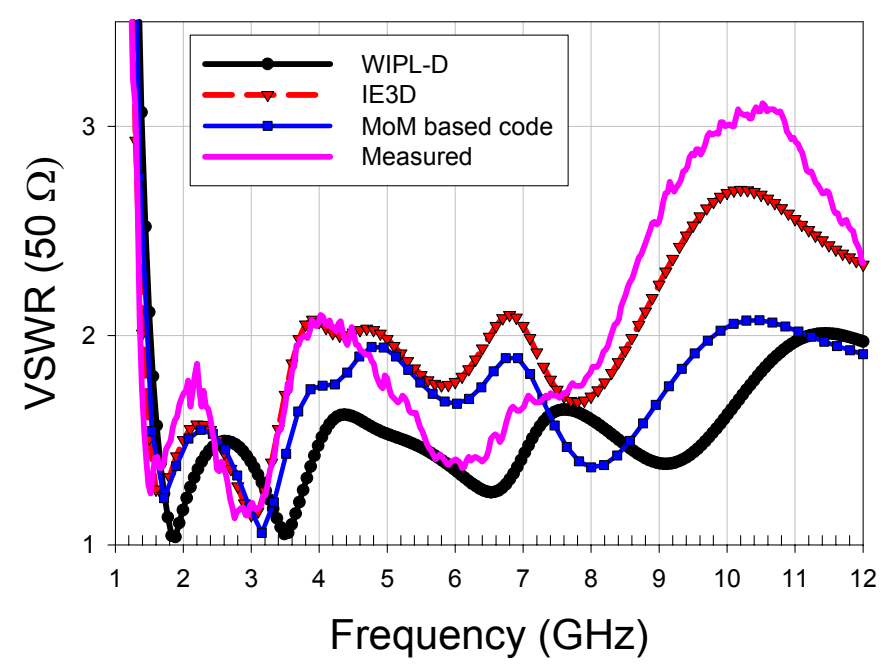

Figure 5.61 Simulated and measured VSWR for the optimised arrowhead monopole.

Figure 5.62 illustrates the normalised radiation patterns in the azimuthal plane ( $X Y$ plane) and in two elevation planes ( $Y Z$ and $X Z$ planes) for the shorted arrowhead monopole at three different frequencies. The asymmetry suffered by the radiation pattern is due to the asymmetry introduced by the shorting pin. Anyhow, the azimuthal radiation pattern is more or less omnidirectional in the whole band. As usual, the cross polarisation level rises as the frequency increases. This can be explained by the increase of the transverse current component in the surface of the monopole. 

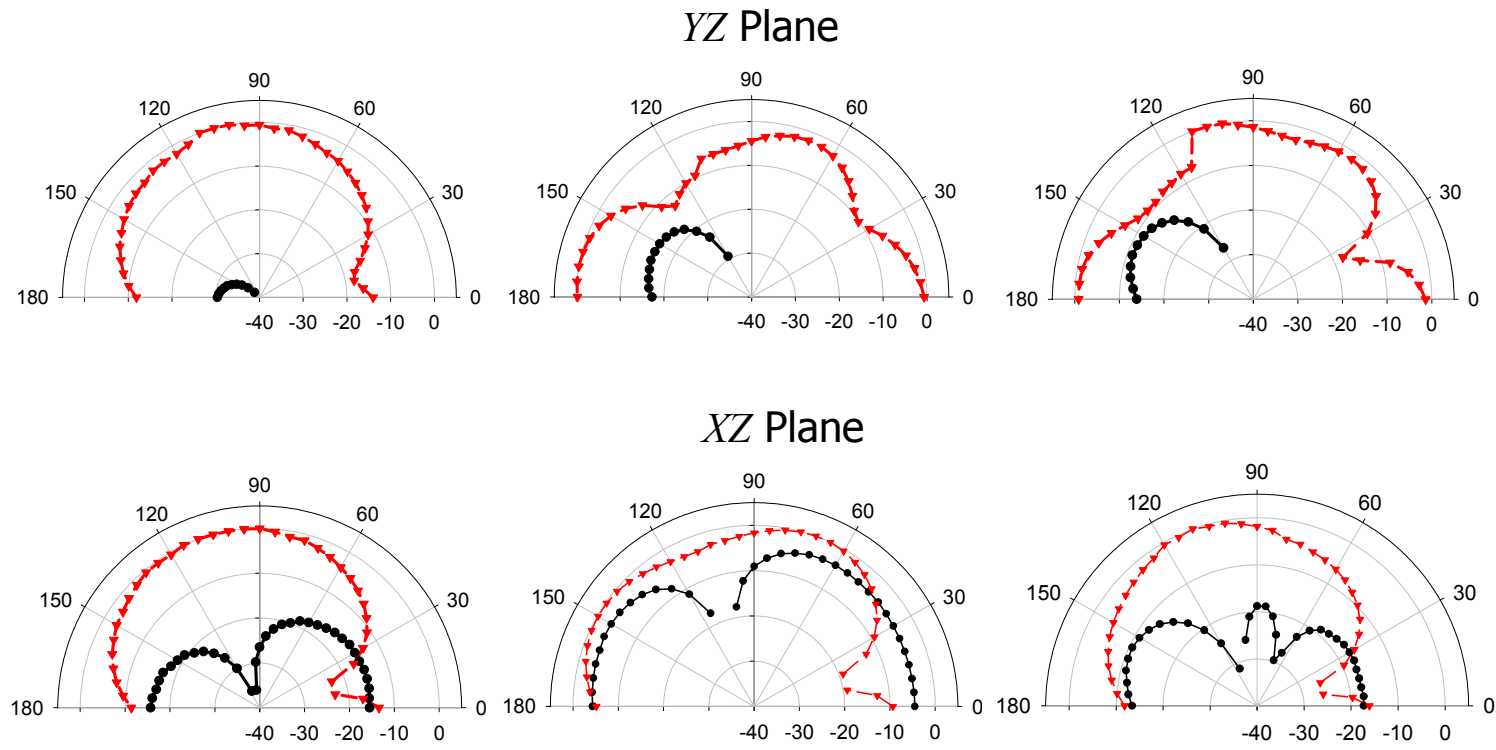

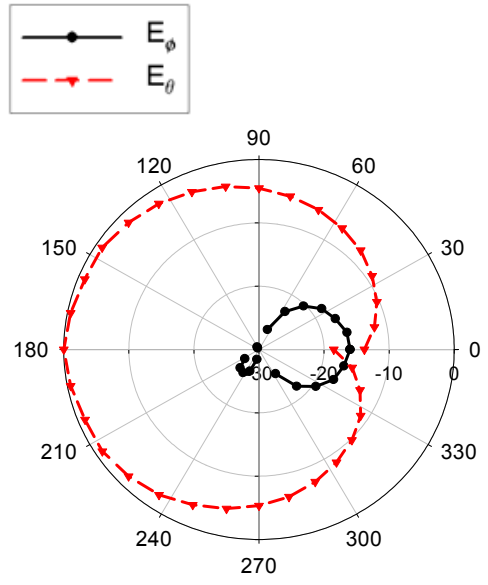

(a) $f=1.8 \mathrm{GHz}$

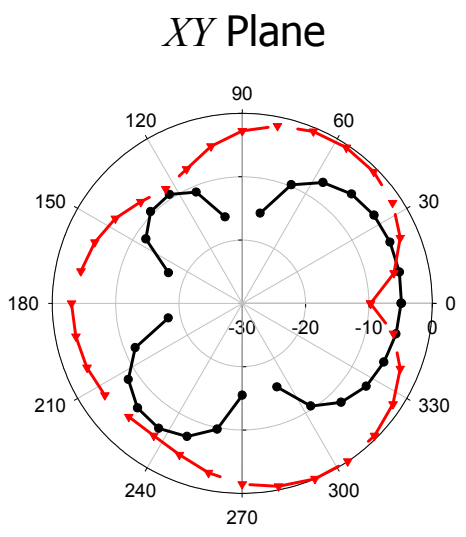

(b) $f=5 \mathrm{GHz}$

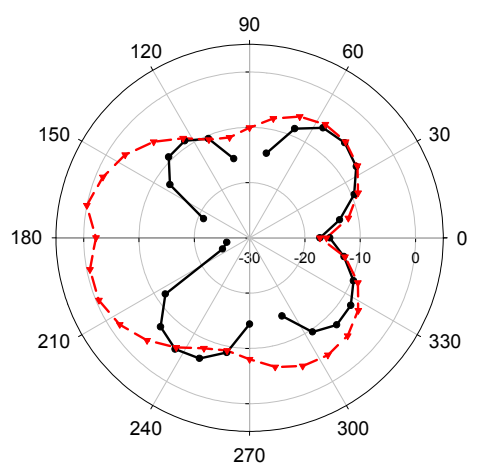

(c) $f=8 \mathrm{GHz}$

Figure 5.62 Normalised radiation patterns in the $Y Z$ plane, $X Z$ plane, and $X Y$ plane, for the arrowhead monopole at three frequencies: (a) $1.8 \mathrm{GHz}$, (b) $5 \mathrm{GHz}$, and (c) $8 \mathrm{GHz}$

In conclusion, after an optimization process, the shorted arrowhead monopole has been demonstrated to be a really compact and wideband planar antenna. This attractive behaviour is based on the combination of different techniques to increase the impedance bandwidth, such as the insertion of a shorting pin, and the bevelling of the base. Due to its compact size the antenna is suitable for handsets and base stations, for wireless systems. Moreover, its broadband behaviour allows multi-service operation, since the antenna covers DCS1800, DECT, PCS1900, UMTS, WLAN, and Bluetooth bands, experimenting little degradation of its radiation patterns. 


\subsubsection{Use of a double feeding configuration to improve the input bandwidth of planar monopoles.}

To explain how the use of a double excitation can improve the impedance bandwidth of a monopole, let us consider a square planar monopole analyzed from image theory point of view. As shown in Figure 5.63 (a) the monopole can be modelled as a planar plate with two narrow slits that account for the feeding gap. In patch antenna design, the insertion of narrow slits at the patch's nonradiating edges is a commonly used technique to obtain compact antennas [7]. The slits force the current to meander, so the resonant frequency decreases. The main problem of this technique is that the current meandering results in a horizontal component of the current that degrades the polarization and bandwidth of the antenna. With the aim of verifying this assessment, Figure 5.63 illustrates the current distribution at resonance for the vertical current mode of a rectangular plate of dimensions $8.5 \mathrm{~cm} \times 4 \mathrm{~cm}$, with and without slits. The vertical current mode of the slitted structure resonates at $1.3 \mathrm{GHz}$, and presents horizontal current flow near the slits, while the vertical current mode of the complete rectangular plate resonates at a higher frequency, $1.6 \mathrm{GHz}$, and displays pure vertical currents. Moreover, currents in the rectangular plate are much more intense than in the one with slits, for the same colour scale.

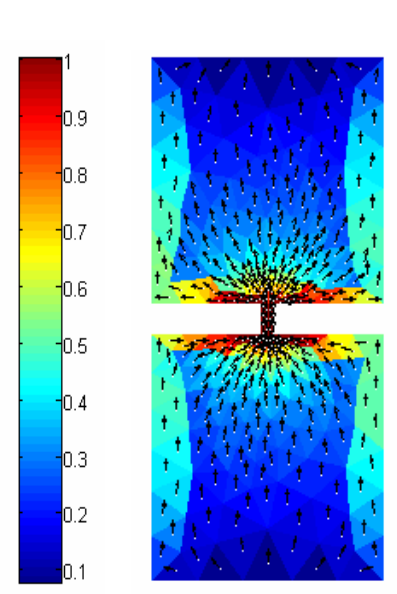

(a) $f=1.3 \mathrm{GHz}$

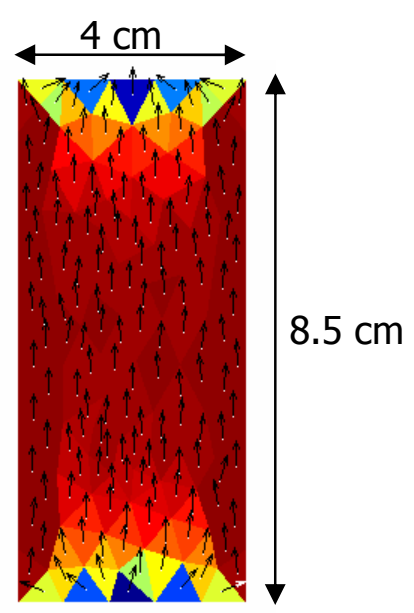

(b) $f=1.6 \mathrm{GHz}$

Figure 5.63 Current distribution at resonance for the vertical current mode of a rectangular plate of dimensions $8.5 \mathrm{~cm} \times 4 \mathrm{~cm}$ : (a) With slits. (b) Without slits.

Let us continue studying the characteristic angle versus frequency curves depicted in Figure 5.64 These curves demonstrate that the vertical current mode of the rectangular plate offers broader radiating bandwidth than the one with slits, since its associated characteristic angle stands near $180^{\circ}$ in a wider frequency range. 
From previous results, it arises that the bandwidth performance of the square monopole would improve if only the existence of vertical currents were allowed. This can be accomplished using the double feeding configuration proposed in [128], which is shown in Figure 5.65 The feeding structure consists in a splitting network connected to two symmetrical ports at the base of the monopole. The symmetry of the ports prevents the excitation of horizontal currents and assures that only the dominant vertical current mode is present in the structure. The square dimension of the monopole is $4 \mathrm{~cm}$, and the dimensions of the splitting network and the position of the ports, have been optimized by E. Antonino for her Phd. Full detail of the dimensions of the splitting network can be found in [128]

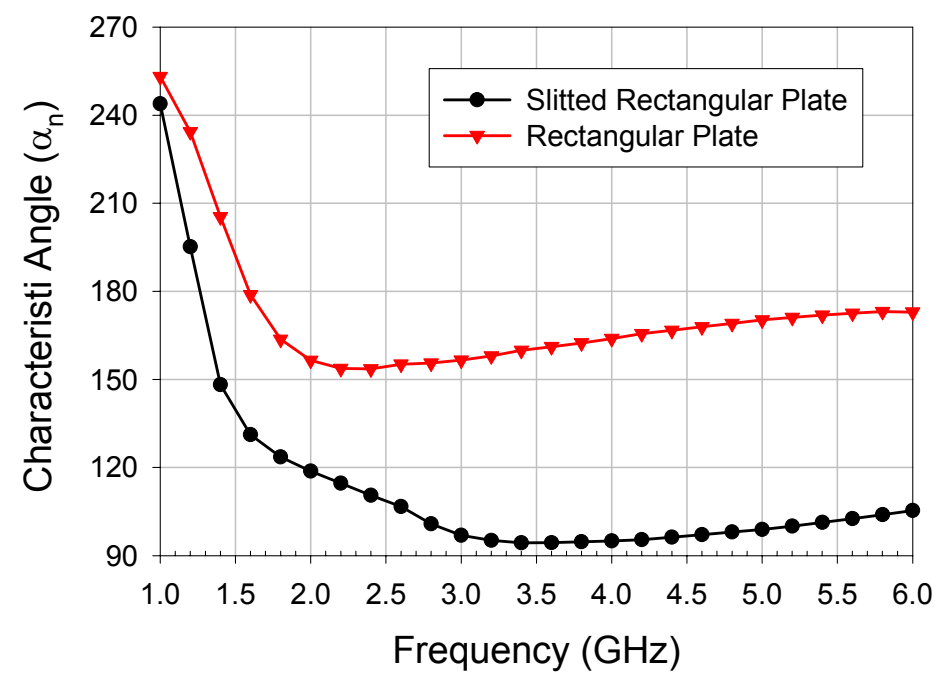

Figure 5.64 Characteristic angle versus frequency curves obtained for the rectangular plates presented in Figure 5.63

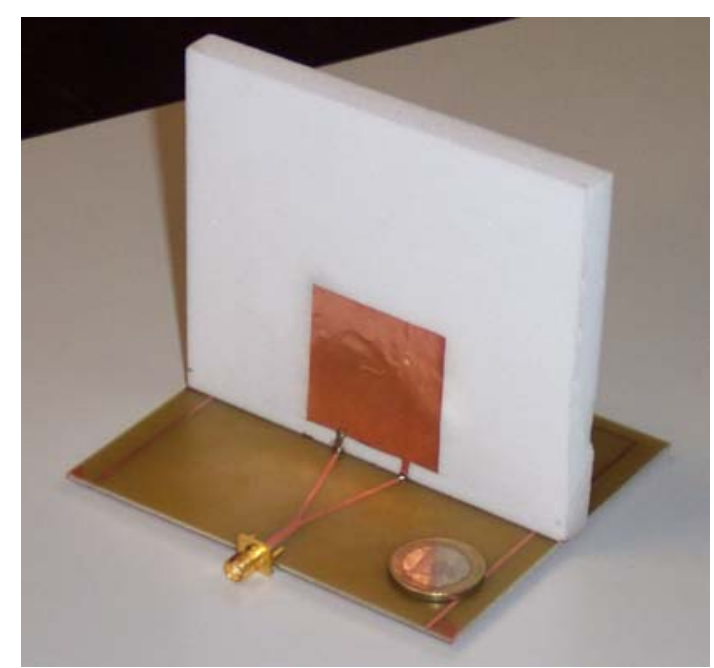

Figure 5.65 Prototype of the square monopole with double feeding configuration. 
To conclude, Figure 5.66 compares the VSWR of the double-fed square monopole to that of a single-fed square monopole of the same dimension. These results for the VSWR have been obtained using the commercial electromagnetic software Zeland IE3D. As it was expected from the previous discussion, when using a double-feed configuration the impedance bandwidth of the square monopole is greatly improved. Figure 5.67 reveals that simulated and measured results for the return loss of the prototype are in good agreement. For brevity, demonstration of the reduction of the cross-polar component of the radiation pattern for the double-fed square monopole has not been included, yet it can be found in [128].

Before finishing this section it should be highlighted that the double fed square monopole presented in [128] has inspired other authors that later have proposed other monopoles based on the same design concept [135]-[136].

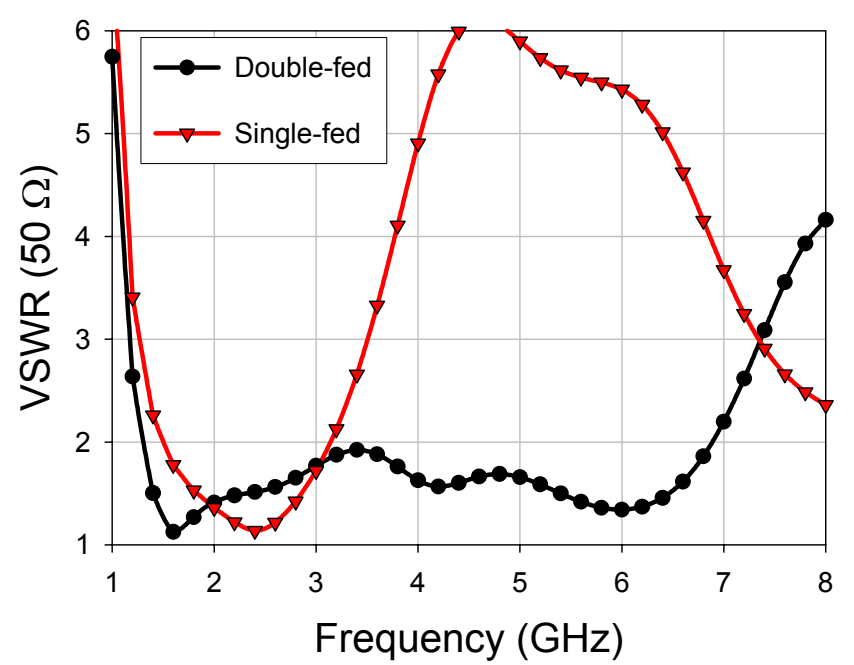

Figure 5.66 Comparison of the VSWR for a double-fed square monopole and a single-fed square monopole of the same dimension.

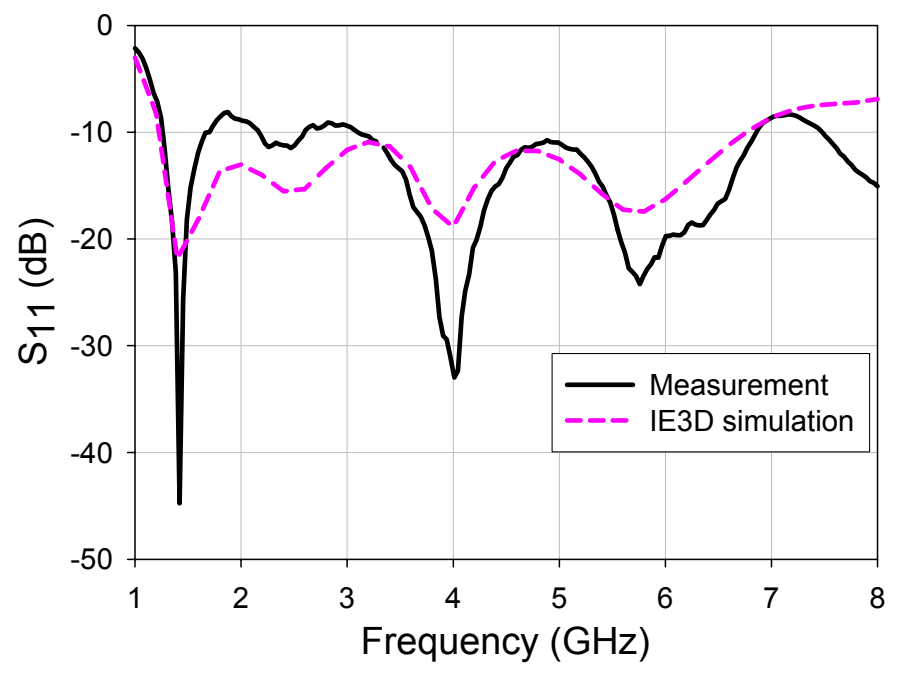

Figure 5.67 Comparison of simulated and measured results for the return loss referred to $50 \Omega$, for the antenna prototype in Figure 5.65 


\subsubsection{Application of planar monopole antennas to MIMO systems.}

Multiple Input Multiple Output (MIMO) systems are a very up-to-date solution to face the growing capacity demand for new wireless communication systems. By using multiple inputs and multiple outputs significant improvement in the system capacity is achieved. Generally, MIMO systems employ multiple antennas at both ends of a wireless link. If the signals received by different antennas are uncorrelated, very high data rates may be reached [137]. The problem is that the volume occupied by multiple antennas is often prohibited, especially for modern compact handsets.

Recent studies have shown that multiple antennas can be replaced by a single multimode antenna, like the archimedian four-arm spiral proposed in [138]. A different approach to reduce system size consists in using compact integrated diversity antennas, such as the one described in [139]. This antenna that is based on a Y-patch, incorporates two antennas into one, and it uses two isolated feed ports to provide diversity signals.

In previous section, a square planar monopole with two symmetrical feeding ports, suitable for UWB systems, has been presented. The thing is that, if the correlation between the two ports of this antenna were small, the ports could be fed independently providing diversity, the same way as the Y-patch proposed in [139]. With this consideration in mind, a long slit cut along the symmetry axis of the antenna was found to be the solution to get the required isolation between the ports. Figure 5.68 shows the geometry of the definitive design. The dimension of the antenna has been optimized to operate at $2.5 \mathrm{GHz}$, and the location and size of both ports have been adjusted to match the connectors' impedance of $50 \Omega$. The dimension of the square radiating element, which is in $X Z$ plane, is $55 \times 55 \mathrm{~mm}^{2}$. The feeding ports are two $2 \times 4 \mathrm{~mm}^{2}$ strips placed symmetrically at $9 \mathrm{~mm}$, on both sides of the slit, whose size is $2 \times 32 \mathrm{~mm}^{2}$. A prototype of the definitive monopole, fabricated at the laboratories of the GRE in UPV, can be seen in Figure 5.69 .

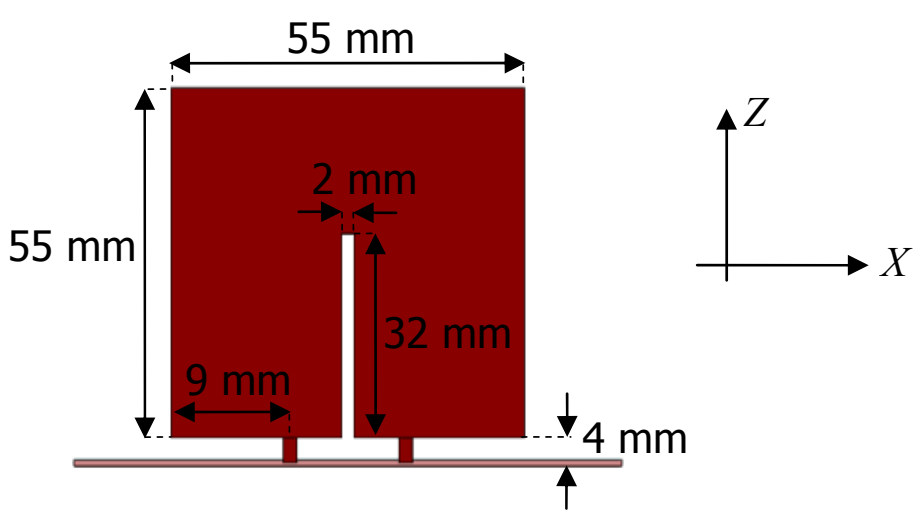

Figure 5.68 Geometry of the definitive design for MIMO applications. 
Figure 5.70 shows the simulated and measured $S$ parameters against frequency for the antenna prototype. Simulations have been performed using IE3D. Simulated and measured results are in fairly good agreement. The structure presents a $S_{11}$ parameter of less than $-10 \mathrm{~dB}$ in a range of frequencies of about $1 \mathrm{GHz}$. Besides, the $S_{12}$ parameter stays below $-20 \mathrm{~dB}$ in most part of this frequency range, taking values below $-35 \mathrm{~dB}$ at the design frequency.

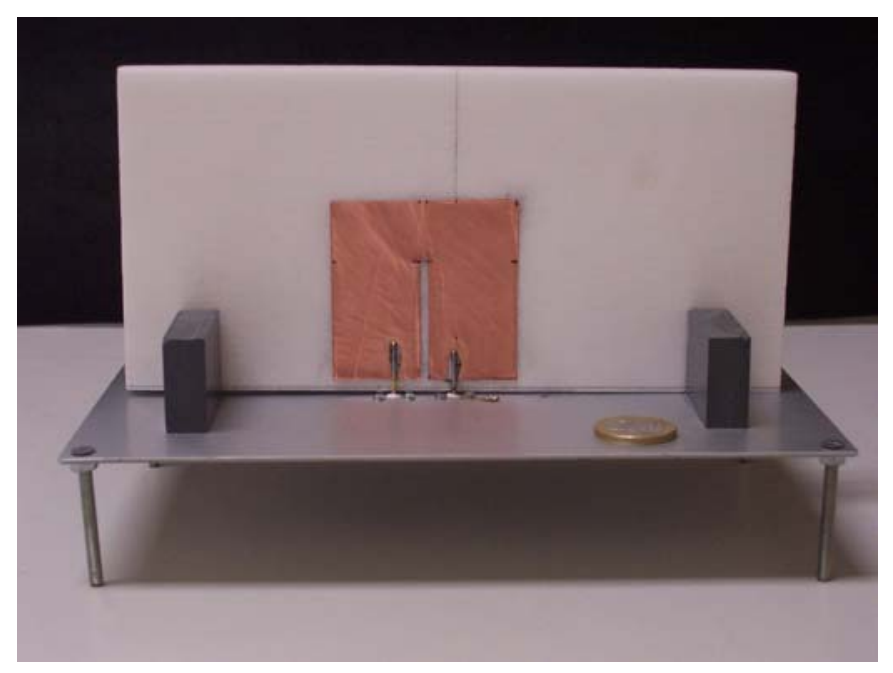

Figure 5.69 Prototype of the square monopole with isolated ports.

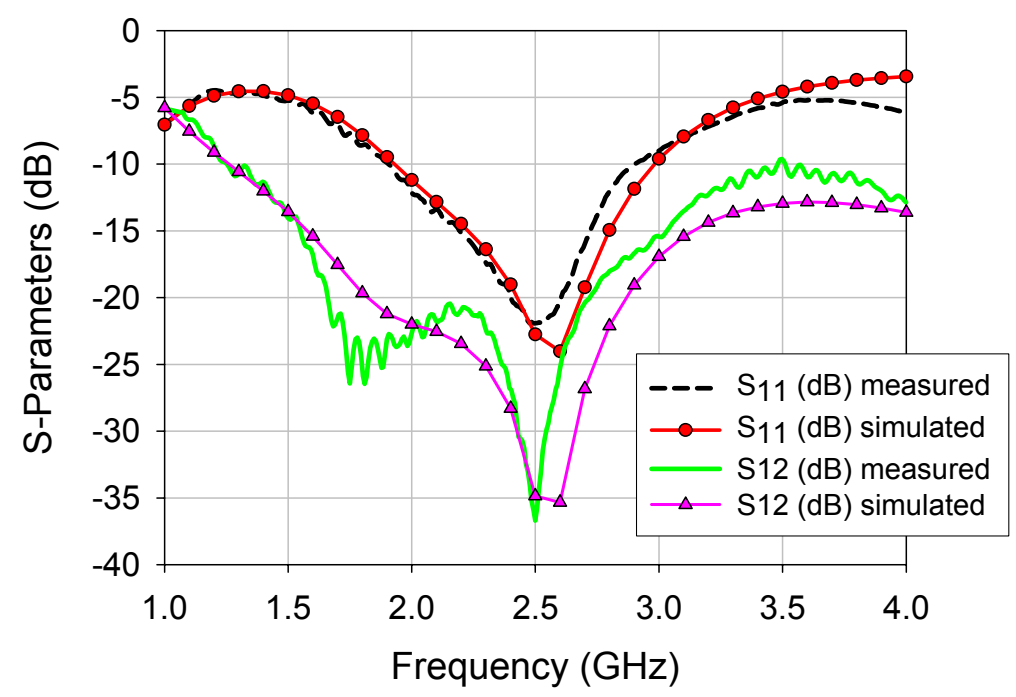

Figure 5.70 Simulated and measured $S$ parameters for the antenna prototype in Figure 5.69

To characterize port isolation in a more precise way, the envelope correlation is calculated from S parameters, as described in [140]. As can be seen in Figure 5.71 the prototype provides correlation values that stay below 0.02 in all the bandwidth of interest. 
Therefore, it seems that this antenna meets the requirements for achieving diversity, and hence to increase the system's capacity. At the same time the antenna presents compact size and wide impedance bandwidth.

Finally, Figure 5.72 shows the gain radiation patterns, computed at $2.5 \mathrm{GHz}$ for this structure using IE3D. These patterns are obtained by exciting port 1 , while terminating port 2 with a $50 \Omega$ load. Due to the symmetry of the system, the patterns are the identical for both ports.

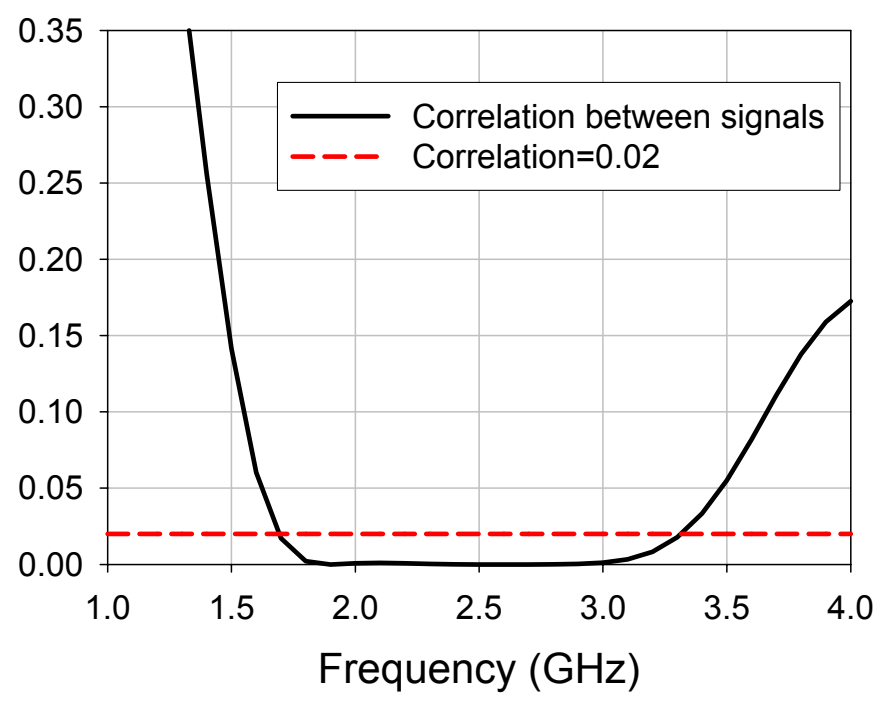

Figure 5.71 Correlation values obtained from the prototype.

(a) $X Y$ Plane

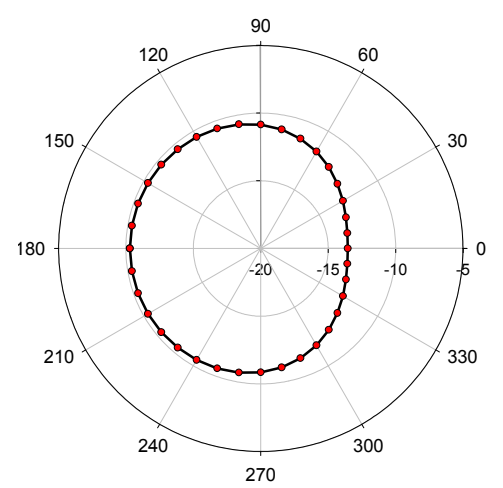

(b) XZ Plane

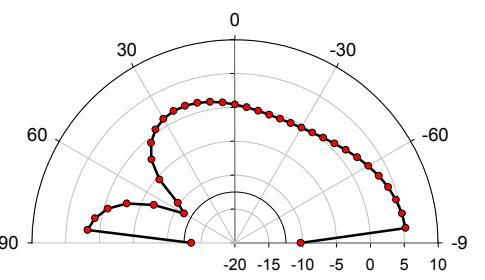

(c) YZ Plane

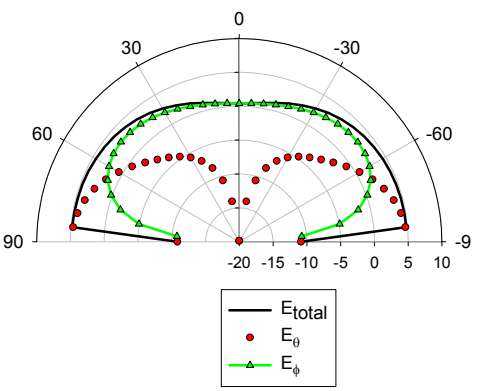

Figure 5.72 Gain radiation patterns at $2.5 \mathrm{GHz}$ computed with IE3D: (a) $X Y$ plane. (b) $X Z$ plane. (c) $Y Z$ plane. The reference angle $0^{\circ}$ corresponds with the $Z$-axis. 


\section{CHAPTER 6. Modal Analysis of Radiating Ground Planes}

\subsection{Introduction}

The fast development of wireless communications and mobile systems in the last decade has stimulated the market demand for novel antenna designs for mobile terminals. However, antennas for actual mobile terminals are subjected to more stringent requirements every time. On one hand, antennas are demanded to operate at different mobile standards which work at different frequency bands, so they may exhibit multi-band or broadband behaviour. On the other hand, due to the rapid advance of integrated circuit technology, mobile handsets are becoming smaller and smaller, so the space available for the antenna is every time more reduced.

Consequently, a lot of investigation has been focused on small antennas, resulting in a great variety of compact wideband, and multiband designs [3]. In general, the actual trend is to implement the antennas internally to the phone, so they are more protected, and less visible than the classical external helixantennas used in the 90's. Among internal compact antennas, the Planar Inverted-F Antenna (PIFA) is the most commonly employed for GSM900/1800 cellular phone handsets [7].

A PIFA antenna is a quarter-wavelength resonator that can be considered as probe-fed shorted patch over an infinite ground plane. Double band [141][142], and triple band operation [143], can be achieved by inserting slits at the radiating path of the PIFA. However, PIFA antennas present an important drawback. As microstrip patches, they are inherently narrow bandwidth antennas.

As a matter of fact, the size of an antenna can not be decreased arbitrarily without affecting other important properties, such as the impedance bandwidth or the efficiency [4]. In general, the performance of compact antennas is limited by the well-known small antennas fundamental limits [5].

Recently, new design strategies have been explored in order to increase the radiation efficiency, and the bandwidth of handset antennas [6]. An example of innovative PIFA design is one that considers the Printed Circuit Board (PCB) of the mobile as part of the antenna [65]. Since the mobile PCB, which acts as the antenna ground plane, presents resonant dimension at mobile frequencies, its shape and size affect the antenna performance in a significant way. In fact, at lowest operating frequencies, the PCB is the main radiator, while the antenna only works as a probe to excite the current modes of the PCB. 
Obviously, to design an antenna from this new perspective, an in-depth knowledge of the current modes of the structure is needed. To that purpose, the Theory of Characteristic Modes, may be very helpful. Thus, the present chapter is going to be focused on the application of characteristic modes to the analysis of different types of radiating ground planes.

The first part of the chapter will be devoted to the study of radiating structures that consist in a folded radiating ground plane. From the current distribution of characteristic modes, it will be demonstrated that the impedance bandwidth of these antennas, can be increased by replacing the classical coaxial probe feeding by a small planar monopole. Moreover, the impedance matching will be improved by inserting slots close to the feeding point. The insertion of these slots forces the current of longitudinal modes to flow through a smaller surface area, so the input resistance is increased.

In the second part of the chapter, the behaviour of notched radiating ground planes will be investigated. The filtering effect due to the interaction between the resonances of the notch and the ground plane will be explained with the information provided by characteristic currents. It will also be demonstrated that, using a radiating ground plane with two notches and two excitation ports, the undesired filtering effect is reduced, and the antenna matching is improved.

\subsection{Design of a folded radiating ground plane antenna for mobile terminals.}

This section describes the procedure carried out to design a handset antenna, based on the PCB resonance design concept, using characteristic modes. It will showed that, characteristic modes not only bring information about the resonance frequency and radiation properties of the current modes of the structure, but also help to select an appropriate location for the probe, to excite the desired current modes flowing on the folded PCB.

\subsubsection{Modal analysis of the basic folded radiating ground plane.}

Figure 6.1 shows the normalized current distribution at first resonance (1.1 $\mathrm{GHz}$ ) for the first six characteristic modes of the basic antenna. The basic antenna can be considered either as a PIFA over a finite ground plane, or as a folded radiating ground plane. Arrows have been plotted together with characteristic currents for a better understanding of the current flow. The dimensions of the antenna are: $L=100 \mathrm{~mm}, W=40 \mathrm{~mm}, W s=35 \mathrm{~mm}, h=10$ $\mathrm{mm}$ and $L_{I}=49.15 \mathrm{~mm}$. Note dimensions $L$ and $W$ coincide approximately with the length and width of the PCB of a common mobile telephone. 
As depicted in Figure 6.1, there are two modes, $J_{01}$ and $J_{02}$, with currents forming closed loops. It will be verified later with the information given by characteristic angles that modes $J_{01}$ and $J_{02}$ are special non-resonant modes. Other modes, such as $J_{1}, J_{3}$ and $J_{4}$, exhibit longitudinal currents along the structure. Mode $J_{l}$ is the fundamental mode, and it flows uninterrupted from the open end on the upper plate to the open end in the lower plate. This fundamental mode resonates when the current path is approximately half a wavelength. Modes $J_{3}$ and $J_{4}$, are higher order longitudinal modes that present one current null, and two current nulls, respectively, along the structure. Finally, mode $J_{2}$ is the only transverse current mode.

The resonance frequency and radiating bandwidth of the above described current modes can be obtained from the characteristic angle curves illustrated in Figure 6.2. It is observed that modes $J_{01}$ and $J_{02}$ do not resonate, and present inductive contribution at every frequency. Longitudinal current modes, $J_{1}, J_{3}$ and $J_{4}$, resonate at $1.1 \mathrm{GHz}, 1.7 \mathrm{GHz}$ and $3.25 \mathrm{GHz}$, respectively, while the transverse mode $J_{2}$ resonates at $3.35 \mathrm{GHz}$.

Table 6.1 comprises the resonant frequencies, the modal quality factors computed using (2.29), and the modal radiating bandwidths obtained by direct inversion of the modal quality factors, for the first resonant modes of the folded radiating ground plane. As expected, the poorest radiating mode is $J_{1}$ since it exhibits the characteristic angle curve with steepest slope at $180^{\circ}$. Modes $J_{2}$ and $J_{3}$ are quite good radiators, while mode $J_{4}$ presents the widest radiating bandwidth.

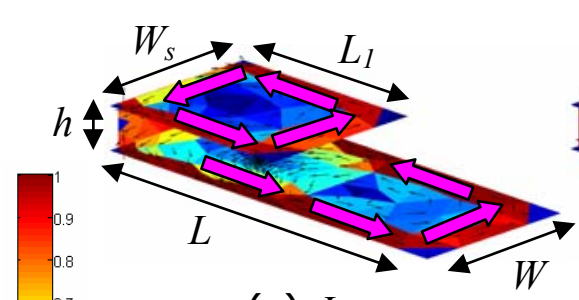

(a) $J_{01}$

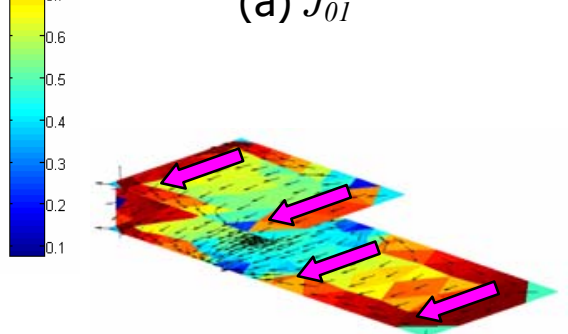

(d) $J_{2}$

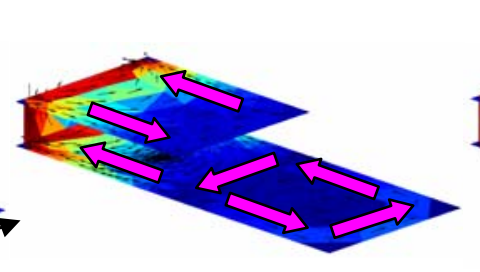

(b) $J_{02}$

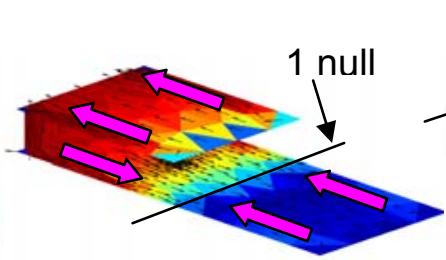

(e) $J_{3}$

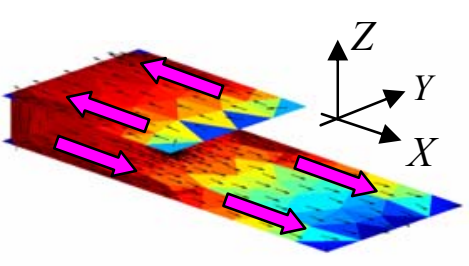

(c) $J_{1}$

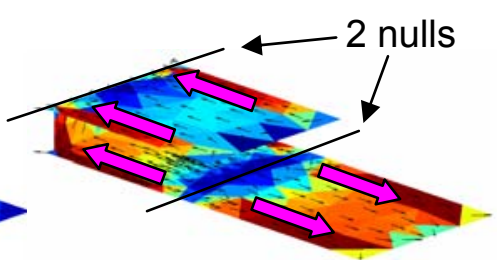

(f) $J_{4}$

Figure 6.1 Normalized current distribution at first resonance $(f=1.1 \mathrm{GHz})$ for the first six characteristic modes of the basic folded radiating ground plane. 


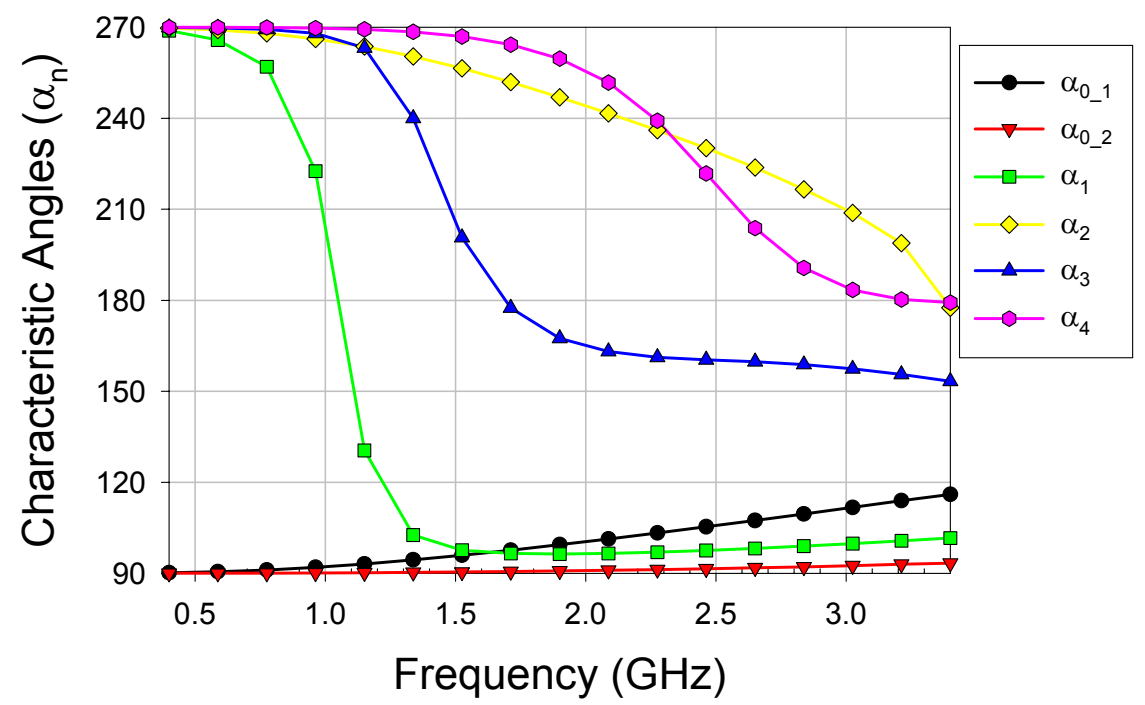

Figure 6.2 Characteristic angle variation with frequency for the first six characteristic modes of the basic folded radiating ground plane.

\begin{tabular}{|c|c|c|c|}
\cline { 2 - 4 } \multicolumn{1}{c|}{} & $f_{\text {res }}$ & $Q_{n, \text { Harrington }}$ & $B W_{n, \text { Harrington }}(\%)$ \\
\hline Mode $J_{1}$ & $1.10 \mathrm{GHz}$ & 6.13 & $16.31 \%$ \\
\hline Mode $J_{2}$ & $3.35 \mathrm{GHz}$ & 3.43 & $29.15 \%$ \\
\hline Mode $J_{3}$ & $1.70 \mathrm{GHz}$ & 1.91 & $52.35 \%$ \\
\hline Mode $J_{4}$ & $3.25 \mathrm{GHz}$ & 0.15 & $>100 \%$ \\
\hline
\end{tabular}

Table 6.1. Resonant frequencies, modal quality factors computed using (2.29), and modal radiating bandwidths, for the first radiating modes of the folded radiating ground plane.

Results presented in Table 6.1 for the modal radiating bandwidth of longitudinal current modes can be more or less explained by simple inspection of the modal current distributions shown in Figure 6.1. As observed, currents in the parallel plate region flow with opposite phase at the lower and upper plates for modes $J_{1}$ and $J_{3}$. This means that there exists some cancellation between these currents flowing like in a transmission line. Conversely, the longitudinal current mode $J_{4}$ presents the broadest radiating bandwidth because its currents flow in phase in the parallel plate region, so its radiation reinforces.

Once the modal analysis of the basic folded structure has been performed, the next step is to select an optimum feeding arrangement to properly excite the desired modes. For the case of a handset antenna for a cellular phone, longitudinal current modes seem to be the most convenient modes to excite, as they resonate close to GSM900, DCS1800, PCS1900 and UMTS operating bands. At the same time these modes present reasonably good radiating bandwidth, in special mode $J_{3}$, that is supposed to provide a matched band wide enough to cover DCS1800, PCS1900, and UMTS services. 


\subsubsection{Determination of the optimum feeding arrangement.}

The optimum feeding configuration should produce a voltage difference in the structure that may favour the appearance of the current distribution of longitudinal modes. The use of a classical coaxial probe feeding has been discarded as it creates a discrete voltage difference between the two points in the lower and upper part of the structure that it connects. This kind of excitation yields narrowband matching, and degrades the current distribution of longitudinal modes, as it increases its transverse current component.

A small planar monopole seems to be a better choice than a coaxial probe, since it creates a distributed voltage difference between the lower and upper plates [144]. Additionally, as it will be shown next, this feeding monopole behaves as a wideband impedance transformer between the feeding port and the upper plate, providing better performance than the classical coaxial probe.

Consequently, as a first instance, a planar rectangular monopole has been chosen to excite the folded radiating ground plane. Figure 6.3 presents the definitive structure and the dimensions of the feeding rectangular monopole that after an optimization process have resulted in: $h=10 \mathrm{~mm}, h_{0}=0.5 \mathrm{~mm}, w_{0}=1.2$ $\mathrm{mm}, w=27 \mathrm{~mm}$ and $L_{f}=72.5 \mathrm{~mm}$. Further details about the antenna design are given in [145].

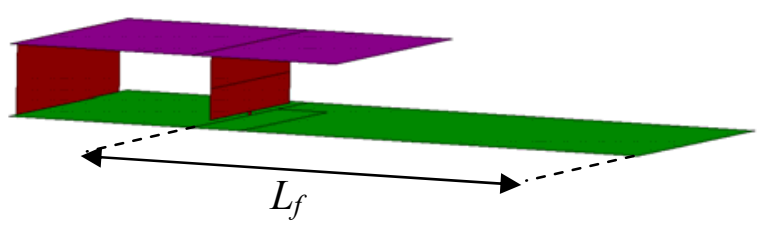

(a)

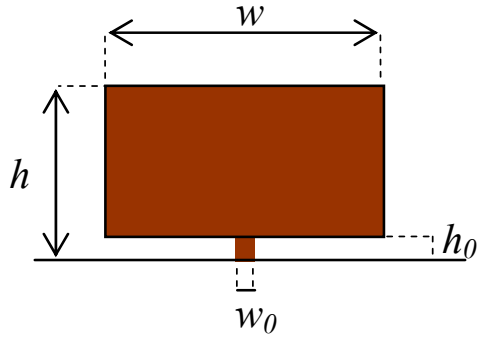

(b)

Figure 6.3 (a) Geometry of the definitive structure. (b) Dimensions of the rectangular feeding monopole.

Nevertheless, when the rectangular feeding monopole is added to the basic structure, it acts as an inductive loading. Hence, the presence of the feeding monopole may alter the resonance frequency and the radiating behaviour of modes. To verify this assessment, characteristic angles of the basic antenna, have been computed again including in the structure the feeding monopole.

Figure 6.4 shows that due to the inclusion of the feeding monopole, the resonant frequencies of our modes of interest, $J_{I}$ and $J_{3}$, are shifted to $1.25 \mathrm{GHz}$ and $2.3 \mathrm{GHz}$, respectively. 
Table 6.2 provides the same information as Table 6.1 , but now including the effect of the feeding monopole. Mode $J_{2}$ has not been included as now, it resonates out of the studied frequency band. Comparing the results in both tables, it is extracted that the radiating behaviour of modes $J_{1}$ and $J_{3}$ improves when the feeding monopole is included. The radiating bandwidth of mode $J_{I}$ is only a bit better, while the one of mode $J_{3}$ duplicates. This is because the highly capacitive nature that modes $J_{1}$ and $J_{3}$ present in the parallel plate region is compensated by the inductive feeding monopole.

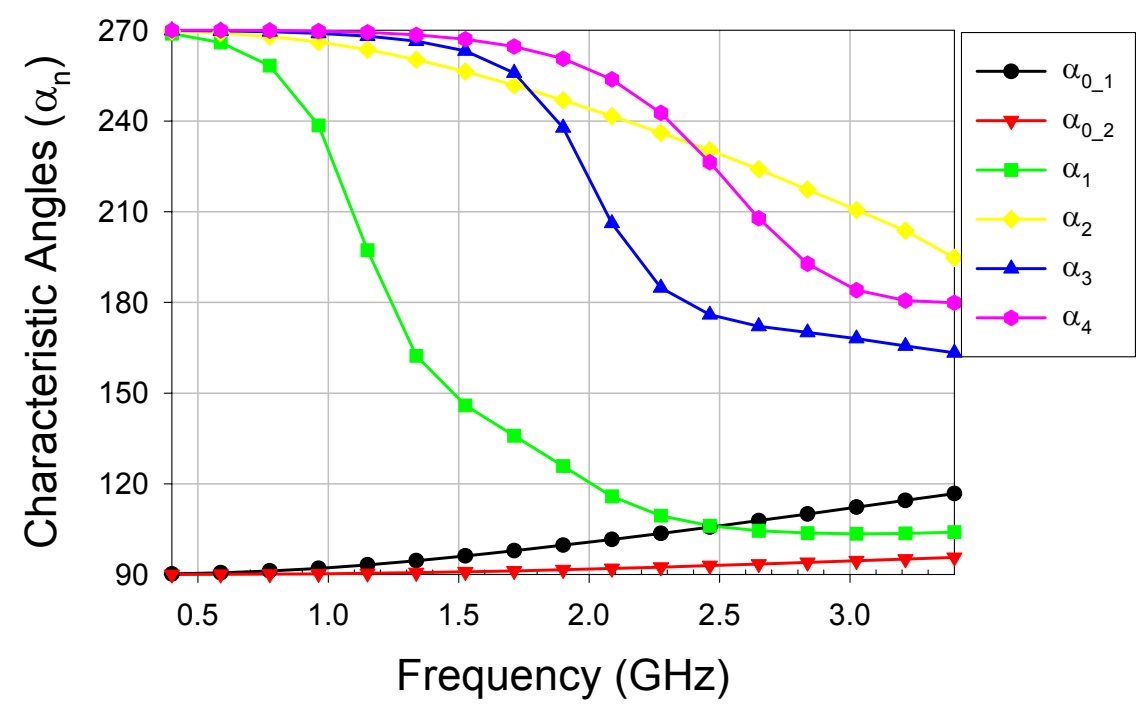

Figure 6.4 Characteristic angle variation with frequency for the first six characteristic modes of the folded radiating ground plane including the effect of the rectangular feeding monopole.

\begin{tabular}{|c|c|c|c|}
\cline { 2 - 4 } \multicolumn{1}{c|}{} & $f_{\text {res }}$ & $Q_{n, \text { Harrington }}$ & $B W_{n, \text { Harrington }}(\%)$ \\
\hline Mode $J_{1}$ & $1.25 \mathrm{GHz}$ & 5.50 & $18.18 \%$ \\
\hline Mode $J_{3}$ & $2.30 \mathrm{GHz}$ & 0.95 & $>100 \%$ \\
\hline Mode $J_{4}$ & $3.35 \mathrm{GHz}$ & 0.22 & $>100 \%$ \\
\hline
\end{tabular}

Table 6.2. Resonant frequencies, modal quality factors computed using (2.29), and modal radiating bandwidths, for the first radiating modes of the folded radiating ground plane including the effect of the rectangular feeding monopole.

Next, in order to find out which modes are excited, the power radiated by the modes $\left(R P_{J_{n}}\right)$ is going to be studied. Figure 6.5 analyzes the contribution of modes to the total radiated power of the antenna $\left(R P_{\text {total }}\right)$. It should be noted that only longitudinal current modes $J_{1}, J_{3}$, and $J_{4}$ are excited. Surprisingly, the maximum radiation of mode $J_{1}$ does not happen at its resonance frequency, $1.25 \mathrm{GHz}$, but at $1.8 \mathrm{GHz}$. 


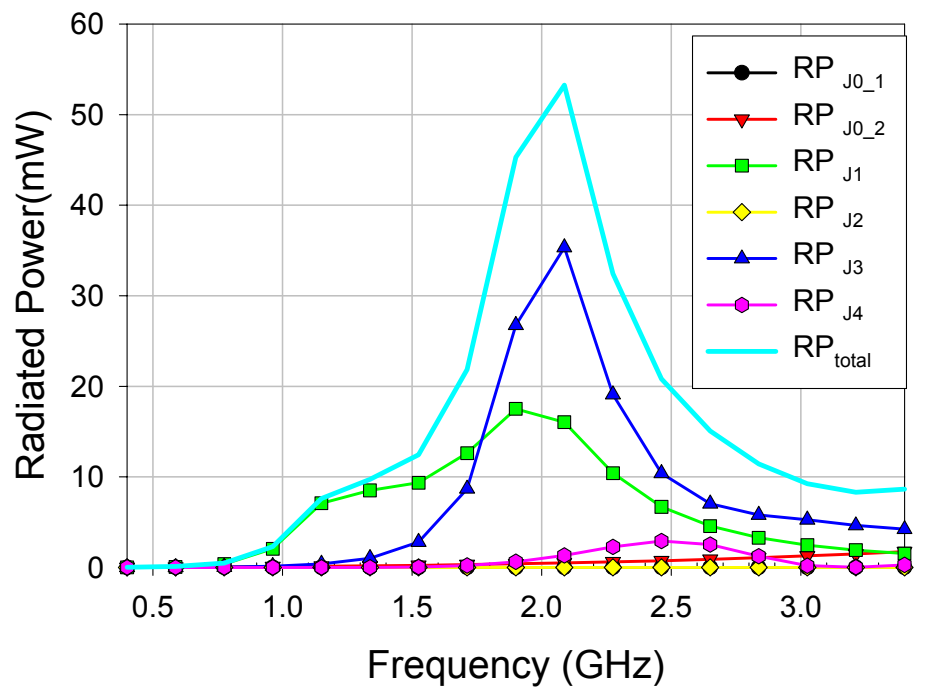

Figure 6.5 Contribution of the different modes to the total power radiated by the folded radiating ground plane excited with a rectangular monopole.

Finally, Figure 6.6 compares the return loss obtained for the antenna using a coaxial feeding probe, and using the rectangular feeding monopole. Both feeding elements have been placed at exactly the same position $L_{f}=72.5 \mathrm{~mm}$. As observed, the rectangular feeding monopole provides wider impedance bandwidth than the coaxial probe, yielding a return loss less than $-6 \mathrm{~dB}$ from $1.15 \mathrm{GHz}$ to more than $4.5 \mathrm{GHz}$.

Classical coaxial probe
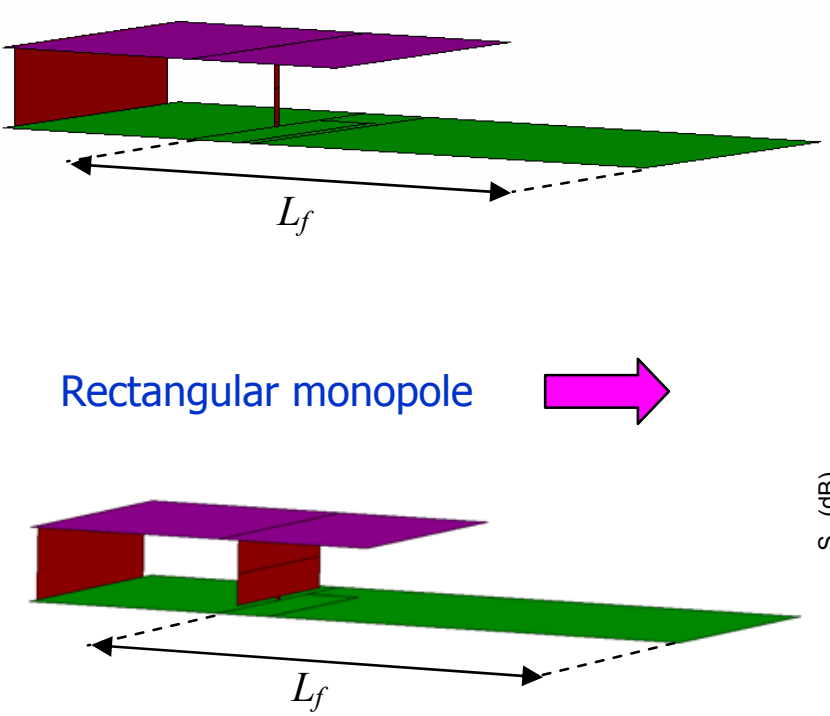
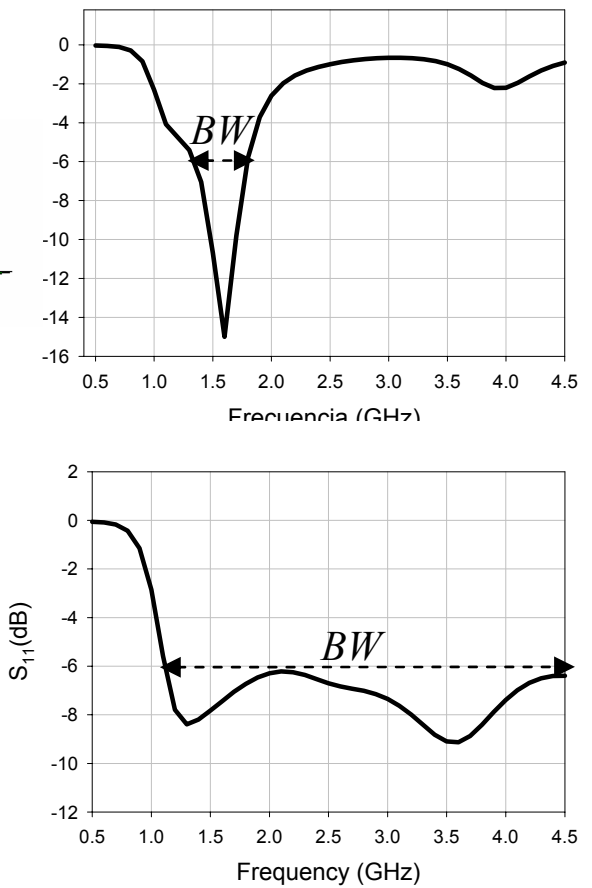

Figure 6.6 Return loss for the folded radiating ground plane when excited with a coaxial probe and with a rectangular monopole. 


\subsubsection{Effect of the insertion of a slot.}

Results in Figure 6.6 confirm that the previously analyzed antenna provides wide-band performance for return loss less than $-6 \mathrm{~dB}$. Note that $-6 \mathrm{~dB}$ is the typical reference value considered in mobile handsets. However, the antenna impedance matching can be improved by inserting a slot in the lower plate of the structure as shown in Figure 6.7. This slot not only produces a meandering effect that reduces the resonant frequencies of longitudinal modes, but also changes the current distribution of these modes near the source, favouring the coupling between them and the feeding monopole.

Figure 6.8 shows the meandering effect experimented by longitudinal current modes due to the insertion of the slot. For brevity, the special non-resonant modes $J_{01}$ and $J_{02}$, which do not contribute to radiation, has not been included here. Mode $J_{5}$, which had not been represented before, is a mixed mode with longitudinal and transverse currents. Note that modes $J_{1}, J_{3}, J_{4}$ and $J_{5}$, present transverse currents flowing with opposite phase in the upper and lower plates, due to the current meandering. In the rest of the structure, the current distributions of modes are very similar to those depicted in Figure 6.1 for the basic folded radiating ground plane.

The reduction in resonance frequency of characteristic modes caused by the current meandering is confirmed by Figure 6.9, which presents the characteristic angles curves associated to the first seven modes of the folded slotted ground plane.

Table 6.3 summarizes the resonant frequencies, modal quality factors, and modal radiating bandwidths, for the resonant modes of the folded slotted radiating ground plane that resonate before $3.5 \mathrm{GHz}$. Observe that the slot increases slightly the radiating bandwidth of modes $J_{1}$ and $J_{3}$, whereas it worsens the radiating behaviour of mode $J_{4}$.

Figure 6.10 analyzes the contribution of each mode to the total power radiated by the antenna $\left(R P_{\text {total }}\right)$. It is observed that the first power maximum, approximately at $1.12 \mathrm{GHz}$, is caused by mode $J_{1}$, the second maximum at 1.8 $\mathrm{GHz}$ is due to the excitation of mode $J_{3}$, and the third maximum at $3 \mathrm{GHz}$ results from the contribution of longitudinal modes $J_{3}$ and $J_{4}$, and mode $J_{5}$. Note also that although transverse mode $J_{2}$ is weakly coupled to the excitation, it contributes to the total power at the highest frequencies.

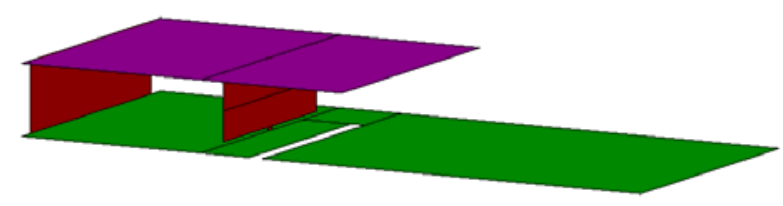

Figure 6.7 Folded slotted ground plane excited with a rectangular monopole. 


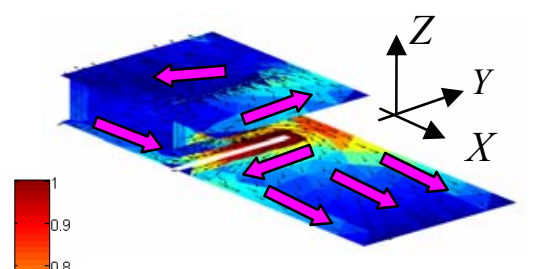

(a) $J_{1}$

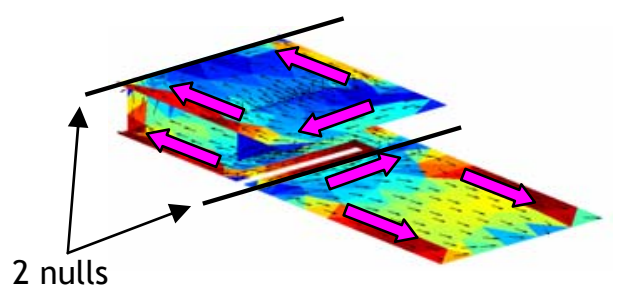

(d) $J_{4}$

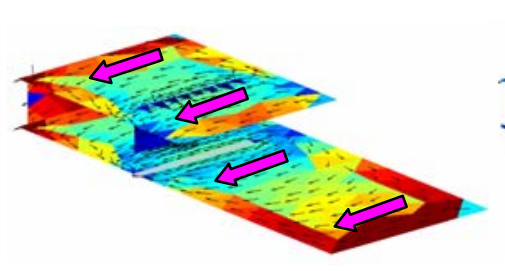

(b) $J_{2}$

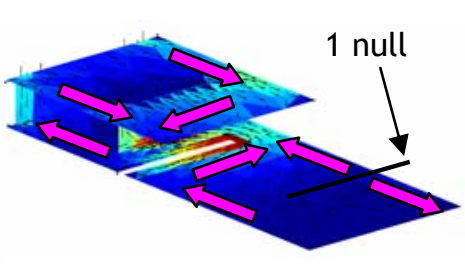

(c) $J_{3}$

Figure 6.8 Normalized current distribution at first resonance $(f=1.1 \mathrm{GHz})$ for the first six characteristic modes of the basic folded radiating ground plane.

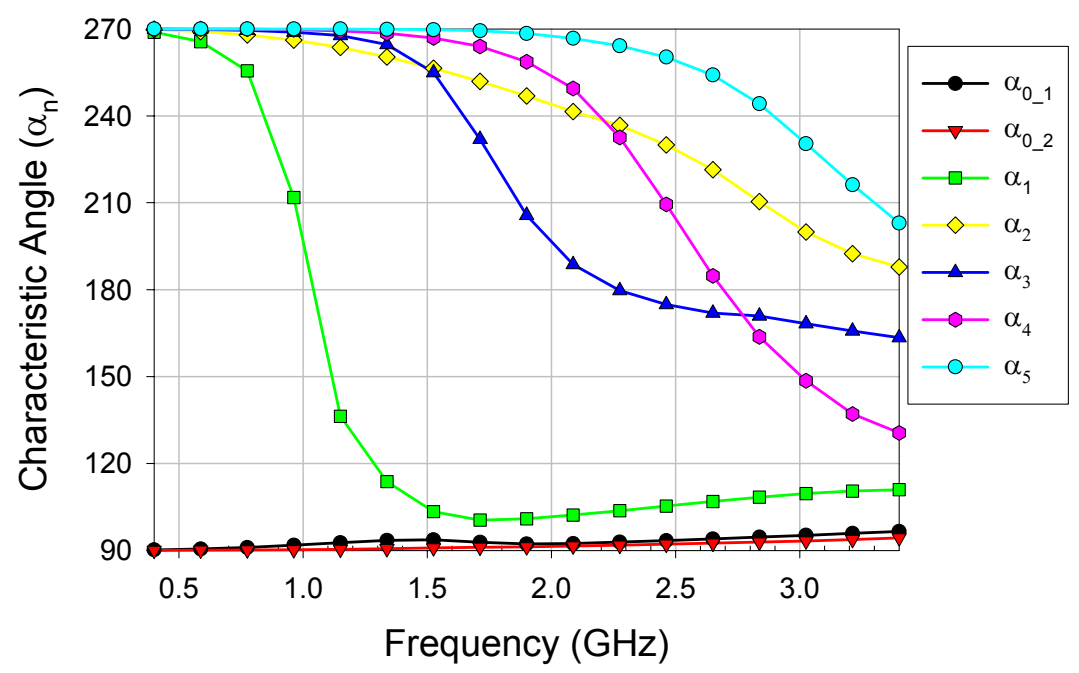

Figure 6.9 Characteristic angle curves for the first six characteristic modes of the folded slotted radiating ground plane fed with a rectangular monopole.

\begin{tabular}{|c|c|c|c|}
\cline { 2 - 4 } \multicolumn{1}{c|}{} & $f_{\text {res }}$ & $Q_{n, \text { Harrington }}$ & $B W_{n, \text { Harrington }}(\%)$ \\
\hline Mode $J_{1}$ & $1 \mathrm{GHz}$ & 4.20 & $23.80 \%$ \\
\hline Mode $J_{3}$ & $2.2 \mathrm{GHz}$ & 0.92 & $>100 \%$ \\
\hline Mode $J_{4}$ & $2.7 \mathrm{GHz}$ & 2.75 & $36.36 \%$ \\
\hline
\end{tabular}

Table 6.3. Resonant frequencies, modal quality factors, and modal radiating bandwidths, for the first radiating modes of the folded slotted radiating ground plane. 
Figure 6.11 sketches the return loss achieved by the folded radiated ground plane fed with the rectangular monopole, with the slot and without the slot. The return loss of the slotted structure presents a clear improvement on the nonslotted one. The insertion of the slot also reduces the lowest limit of the first matched band, at the same time as it improves the matching in the whole frequency band that ranges from $0.9 \mathrm{GHz}$ to $5 \mathrm{GHz}$. As observed, the three discernible matched bands for the slotted antenna correspond with the power maxima at Figure 6.10.

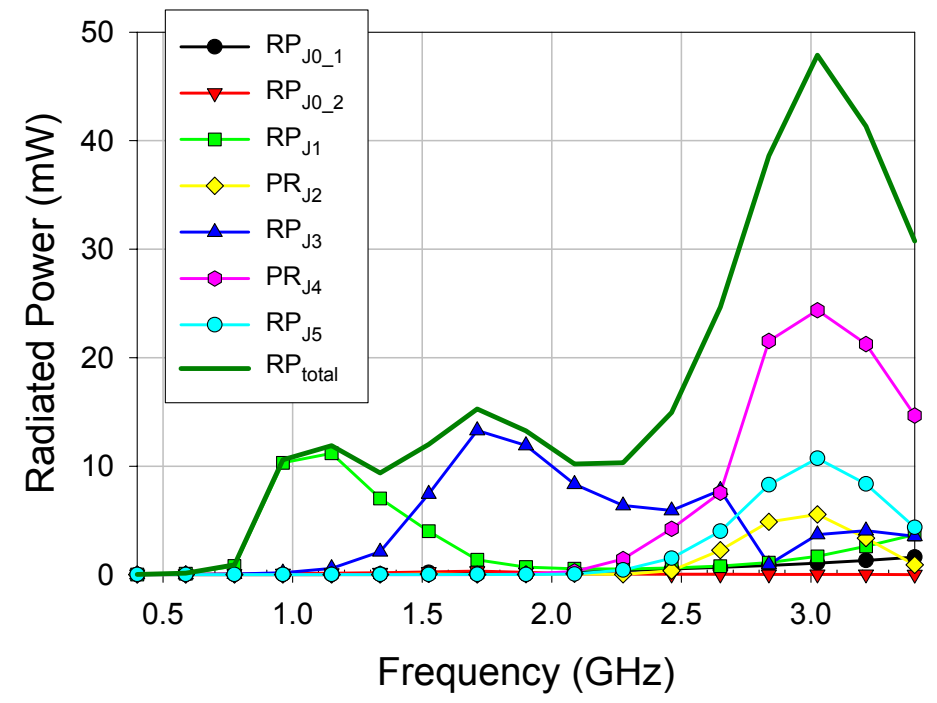

Figure 6.10 Contribution of the different modes to the total power radiated by the folded slotted radiating ground plane fed with a rectangular monopole.

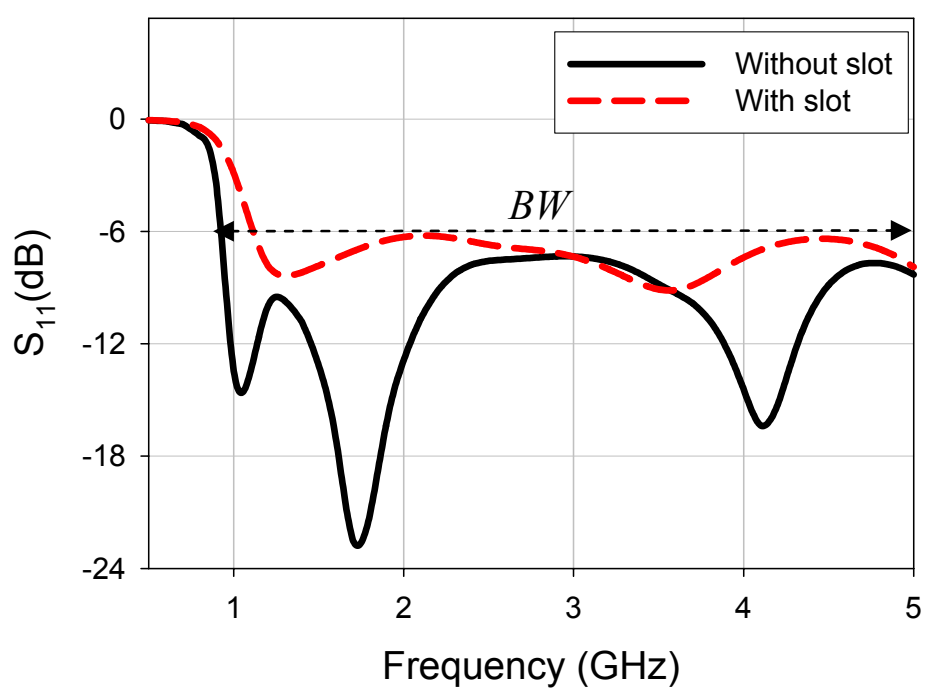

Figure 6.11 Return loss achieved by the folded radiated ground plane fed with the rectangular monopole, with the slot and without the slot 
To validate the simulated results, a prototype of the antenna has been fabricated at the laboratories of the GRE in UPV. A photograph of this prototype can be seen in Figure 6.12. Figure 6.13 illustrates that the return loss simulated with IE3D and measured for the antenna prototype are in good agreement at lowest frequencies. As observed, the antenna is well matched at GSM900, DCS1800, PCS1900, and UMTS operating bands.

Figure 6.14 shows the radiation patterns in the $Z Y$ and $X Y$ planes at $900 \mathrm{MHz}$, and $1800 \mathrm{MHz}$. The omnidirectional behaviour observed at both bands, together with the excellent wideband performance, makes the antenna a good candidate for mobile handsets.

Finally, to evaluate if the matching of the proposed antenna will be altered by the presence of the battery of the mobile terminal, a commercial mobile battery has been added to the antenna prototype as shown in the setup of Figure 6.15 (a). Figure 6.15 (b) shows that the return loss of the antenna has not changed very much because of the addition of the battery. For a return loss of less than $-6 \mathrm{~dB}$, the upper limit of the matched band has been reduced. However, in general the matching levels of the band have improved due to the effect of the battery.

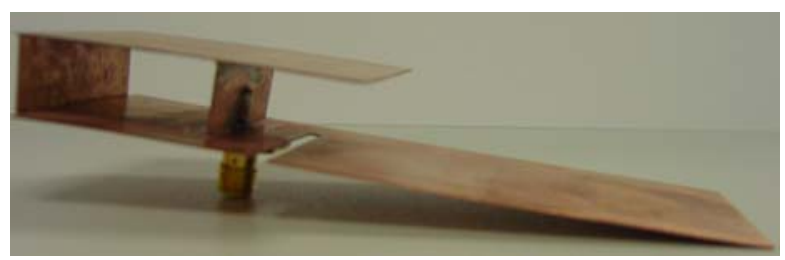

Figure 6.12 Prototype of the folded slotted radiating ground plane fed with a rectangular monopole.

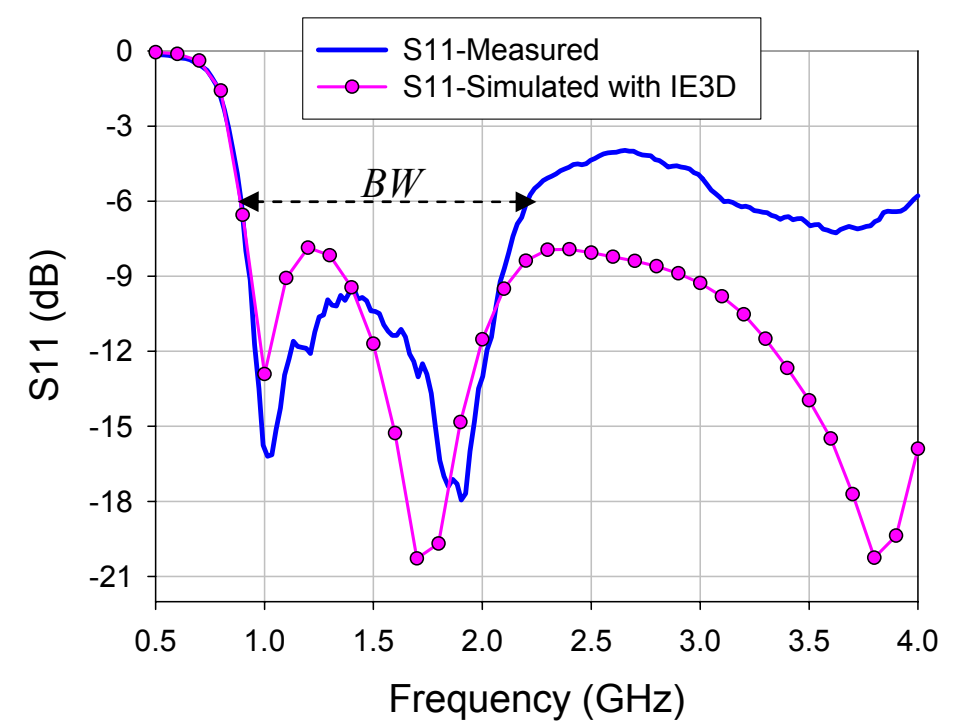

Figure 6.13 Simulated and measured return loss for the antenna prototype. 

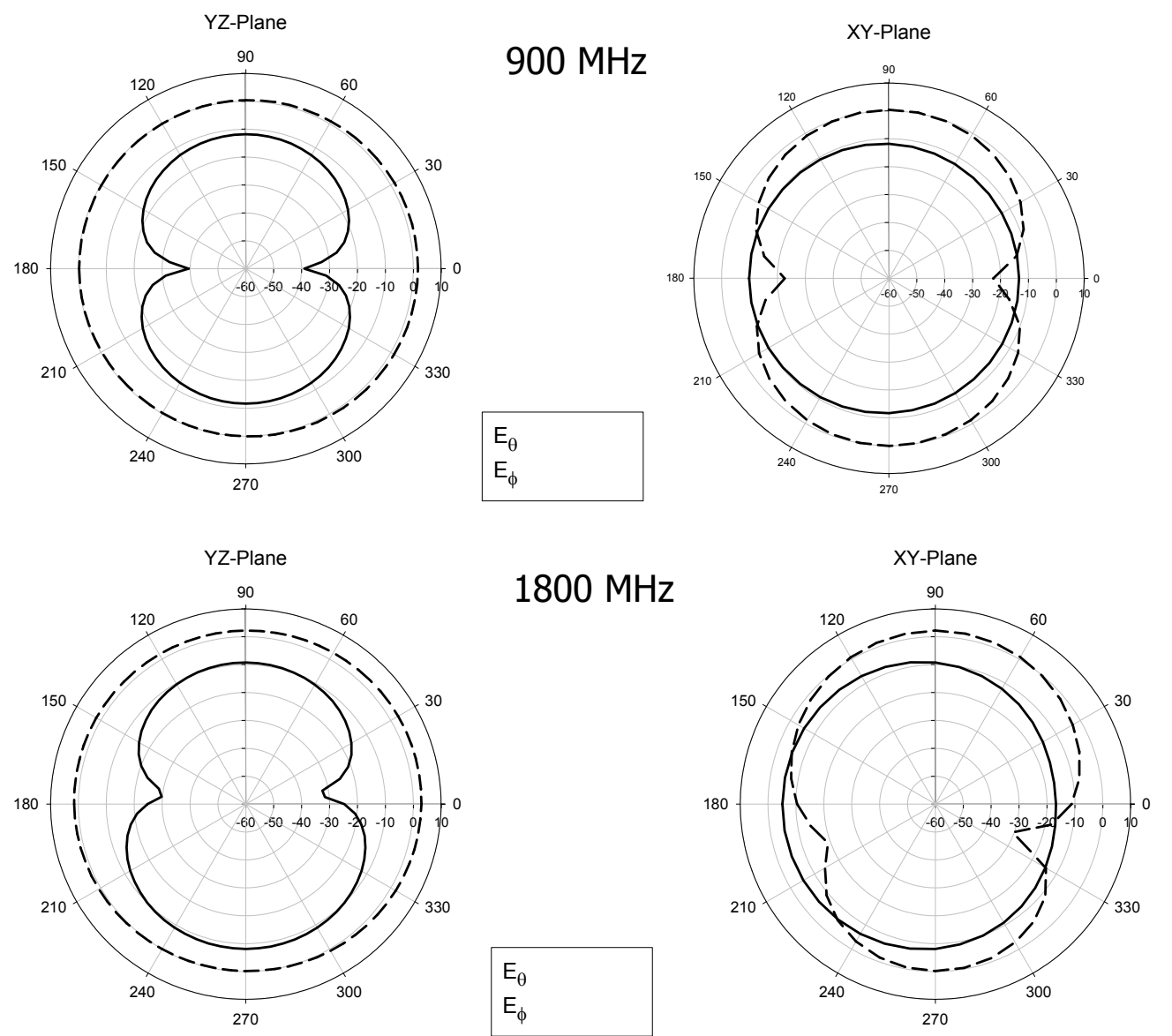

Figure 6.14 Radiation patterns in the $Y Z$ and $X Y$ planes for the folded slotted radiating ground plane at $900 \mathrm{MHz}$ and $1800 \mathrm{MHz}$.

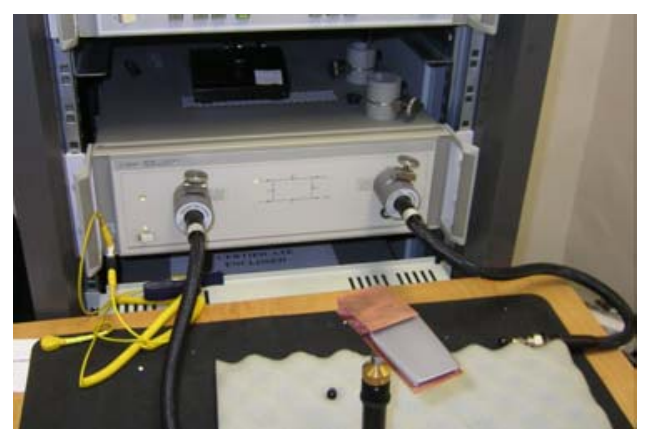

(a)

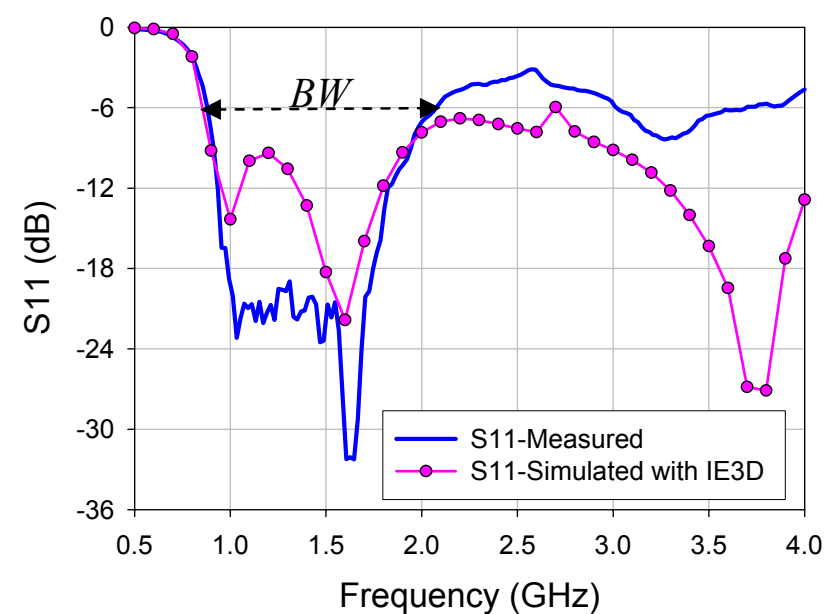

(b)

Figure 6.15 (a) Setup to measure the effect of the battery on the antenna. (b) Simulated and measured return loss for the antenna prototype including the battery. 


\subsubsection{Use of a bow-tie shaped feeding monopole.}

In section 6.2.2 a rectangular monopole has been proposed for feeding the folded radiating ground plane. The choice of this monopole shape was based on its simplicity. However, any other monopole shape could have been used.

In this section, a bowtie shaped monopole has been selected to excite the folded radiating ground plane. The advantage of the bowtie monopole is that it has more parameters to adjust than the square or the rectangular monopole, and therefore, it is easier to achieve maximum matching using this shape.

Figure 6.16 presents the optimized dimensions for the feeding bowtie monopole, which have resulted in: $h=10 \mathrm{~mm}, h_{l}=h_{2}=4.75 \mathrm{~mm}, w_{p}=1 \mathrm{~mm}$, $w_{1}=16 \mathrm{~mm}$ and $w_{2}=32 \mathrm{~mm}$. Like with the rectangular monopole, the addition of the feeding bowtie monopole, shifts the resonant frequency of longitudinal modes $J_{I}$ and $J_{3}$ to higher frequencies, and hence far from GSM900, DCS1800 and UMTS operating bands. Thus, it is necessary to insert a slot to the antenna again, in order to reduce the resonant frequencies of longitudinal modes $J_{1}$ and $J_{3}$. In this case, as shown in Figure 6.17, two slots of width $2 \mathrm{~mm}$ and length 25 $\mathrm{mm}$ have been added to the structure to achieve the desired compactness. The rest of dimensions are $L_{f}=72.5 \mathrm{~mm}, R_{l}=48 \mathrm{~mm}$, and $R_{2}=65.25 \mathrm{~mm}$.

Figure 6.18 shows photographs of a prototype of the antenna, and 0 compares the return loss measured for the prototype with the return loss simulated with IE3D. Observe that measured and simulation agree very well at the lowest frequencies. The return loss is less than $-6 \mathrm{~dB}$, from $0.9 \mathrm{GHz}$ to 4.5 $\mathrm{GHz}$, so the antenna operates at GSM900, DCS1800, PCS1900, UMTS and 802.11.g bands.

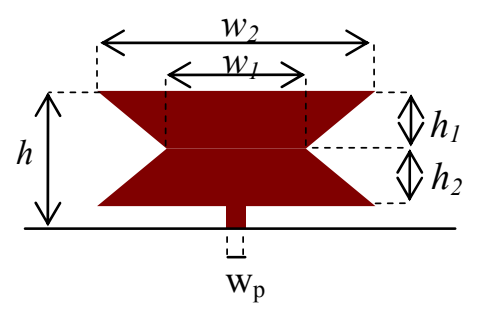

Figure 6.16 Dimensions of the feeding bowtie monopole.

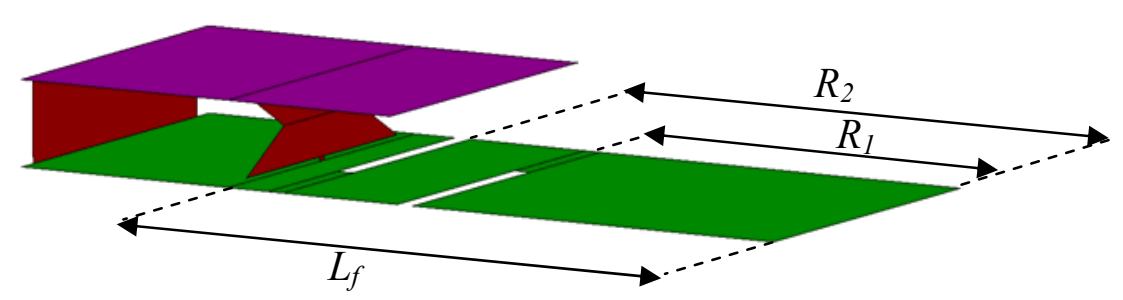

Figure 6.17 Geometry of the folded radiating ground plane with bowtie shaped feeding monopole. 

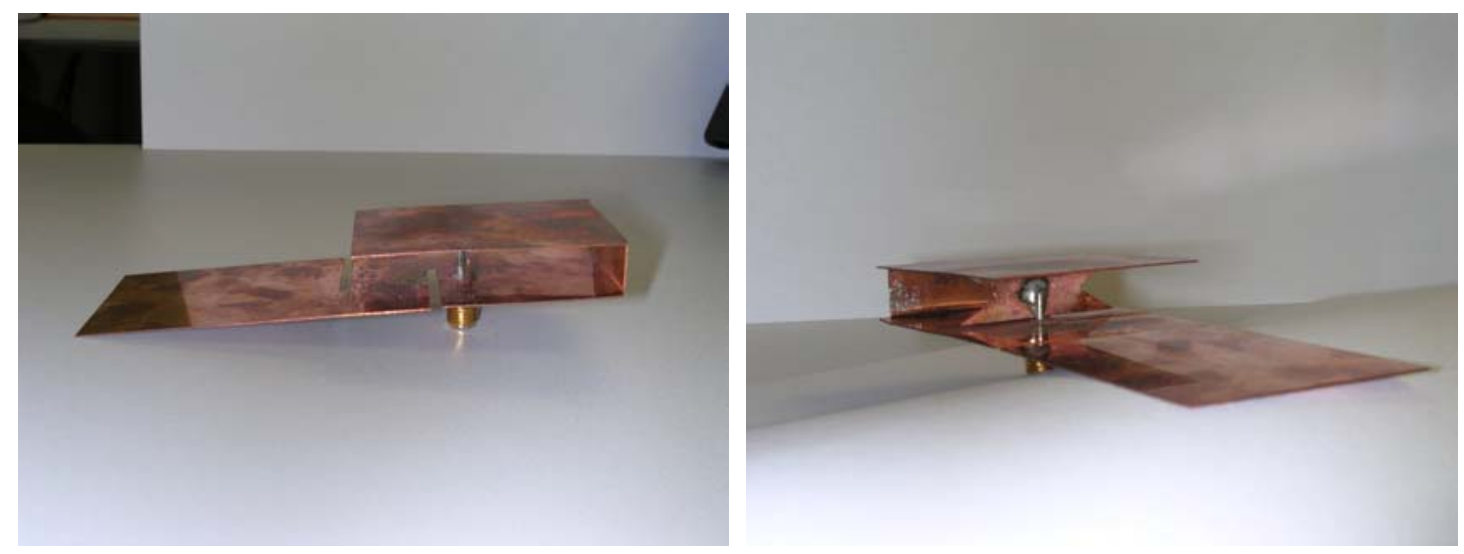

Figure 6.18 Photographs of the prototype of the slotted folded radiating ground plane fed with a bow-tie shaped monopole.

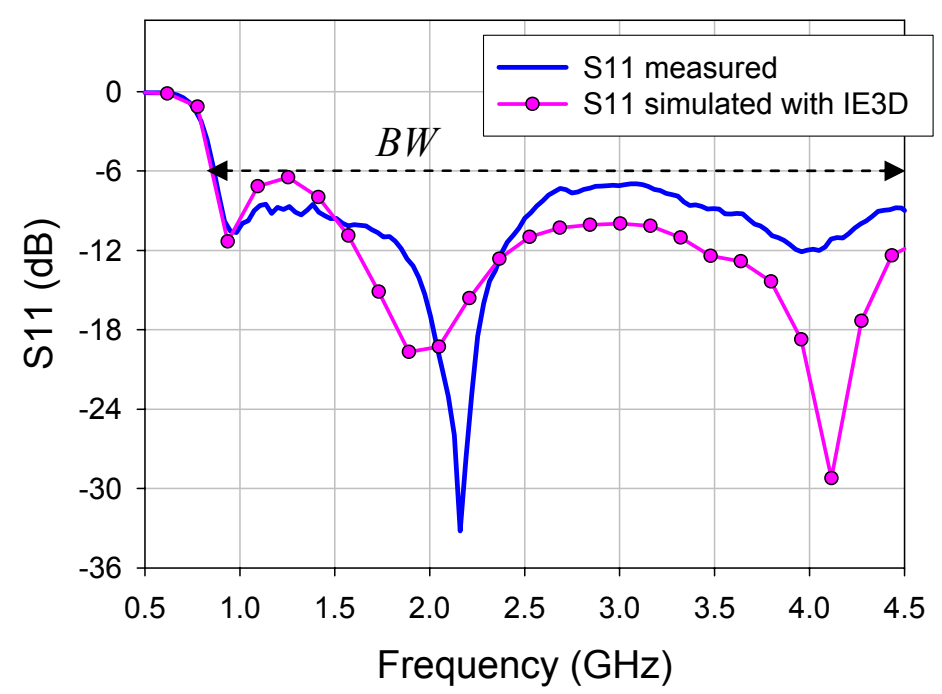

Figure 6.19 Simulated and measured return losses of the antenna prototype in Figure 6.18.

\subsubsection{Conclusions}

An example showing how to use the Theory of Characteristic Modes for controlled antenna design, based on the PCB resonant concept, has been presented with the aim of demonstrating, that characteristic modes are really helpful for the design and optimization of handset antennas. It has been shown that having in mind the current distribution of modes, the geometry of the antenna can be modified to accomplish the desired specifications, while an appropriate feeding configuration can be selected in order to excite the desired modes. 


\subsection{Design of notched radiating ground planes.}

Notch antennas have long been used for HF communications, VHF telemetry, and command on aircraft and missiles. However, self-resonant notch antennas fabricated using microstrip technology, are very compact structures that can be effectively used for wireless communications [146]-[148]. Typically, notch antennas consist in a quarter wavelength slot cut on the edge of a semi-infinite ground plane. They are commonly fed from a coaxial cable without the need of any balancing system, as depicted in Figure 6.20.

However, when a notch is cut on a finite ground plane of resonant dimension, the shape and size of the ground plane significantly affect the performance of the notch antenna. It can be demonstrated that sometimes the interaction between the resonances of the notch and the ground plane results in a filtering effect at some frequencies. Next, different configurations of notch antennas on finite ground plane are going to be analyzed using the theory of characteristic modes.

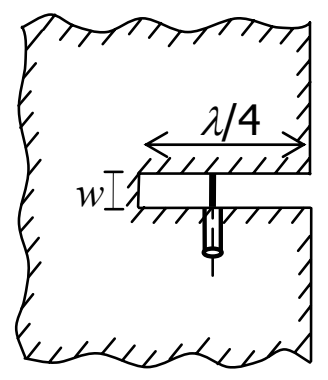

Figure 6.20 Typical quarter wavelength notch antenna cut on the edge of a semi-infinite ground plane, and fed from a coaxial cable.

\subsubsection{Radiating ground plane with a notch.}

Let us consider a notch of length $L_{1}=4.5 \mathrm{~cm}$, and width $W_{1}=1 \mathrm{~cm}$, cut on a metallic ground plane of dimensions $L=10 \mathrm{~cm}$, and $W=6.5 \mathrm{~cm}$. The size of the ground plane has been chosen that of a typical Personal Digital Assistant (PDA). Figure 6.21 sketches the normalized current distribution at $1.2 \mathrm{GHz}$ for the first six characteristic modes of this structure. Mode $J_{0}$ is a special non-resonant mode, with currents forming a closed loop around the perimeter of the ground plane. Mode $J_{l}$ is the fundamental mode, and its current flows from the upper right corner to the lower left corner. Mode $J_{2}$ is a horizontal current mode. Mode $J_{3}$ is a mixed mode with vertical and horizontal currents. Modes $J_{4}$ and $J_{5}$ are higher order vertical modes, with a current null at the centre of the ground plane, and two current nulls, respectively. 
As observed, modes $J_{0}, J_{1}, J_{3}$ and $J_{5}$ present intense currents flowing around the notch. Unfortunately, these intense currents do not contribute to radiation as they are in opposite phase, like in a transmission line, and they cancel each other out along the notch. In contrast, the notch has little effect on the current distribution of mode $J_{2}$, whose currents flow in phase along the whole ground plane, and on the current distribution of mode $J_{4}$, which presents a current null just at the notch.

Figure 6.22 presents the variation with frequency for the characteristic angles associated to the current modes of Figure 6.21. As it had been anticipated, mode $J_{0}$ is a special non-resonant inductive mode, whose characteristic angle stays below $180^{\circ}$ at every frequency. Fundamental mode $J_{l}$ exhibits the narrowest radiating bandwidth, while the rest of modes are all quite efficient radiators as its characteristic angles keep close to $180^{\circ}$ in a wide range of frequencies.

For the sake of completeness, Table 6.4 summarizes the resonant frequencies of all the resonant modes. It is not worth presenting the modal quality factors, nor the modal radiating bandwidths, since all modes except $J_{1}$ present quality factors of less than 1, and hence radiating bandwidths bigger than $100 \%$.

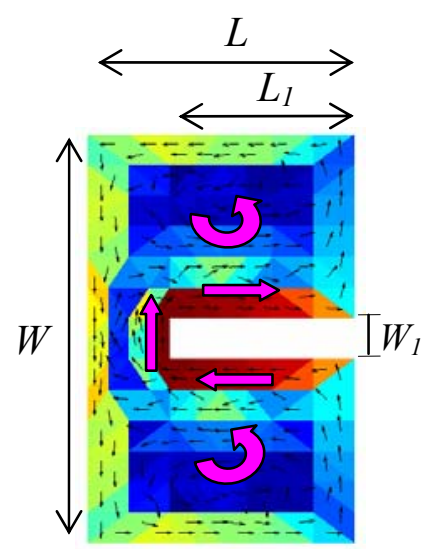

a) $J_{0}$

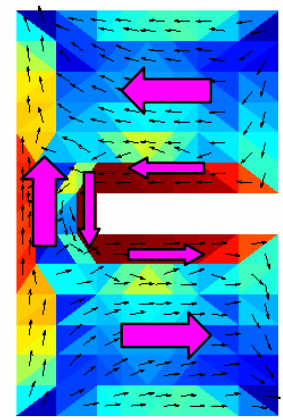

d) $J_{3}$

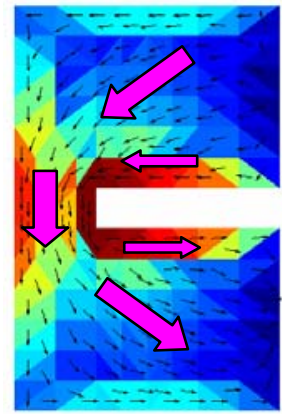

b) $J_{1}$

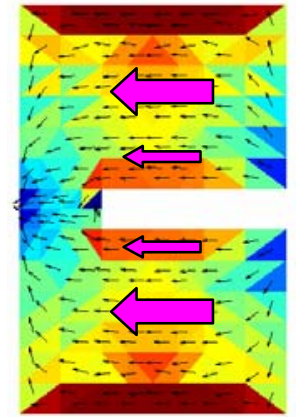

c) $J_{2}$

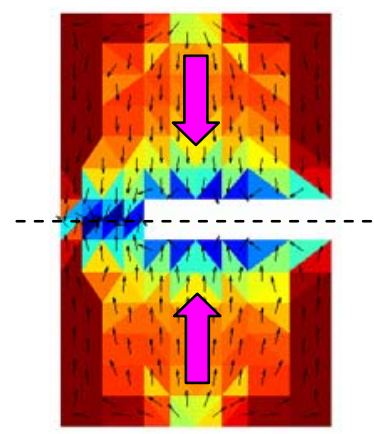

e) $J_{4}$

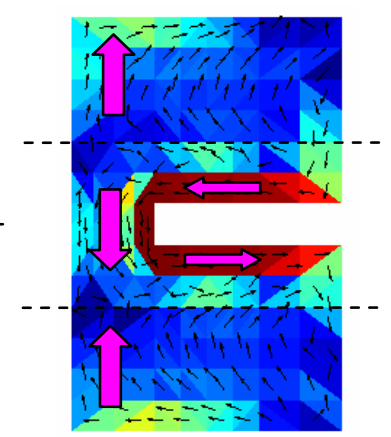

f) $J_{5}$

Figure 6.21 Normalized current distribution at $1.2 \mathrm{GHz}$ for the first six modes of a the radiating ground plane with a notch. 


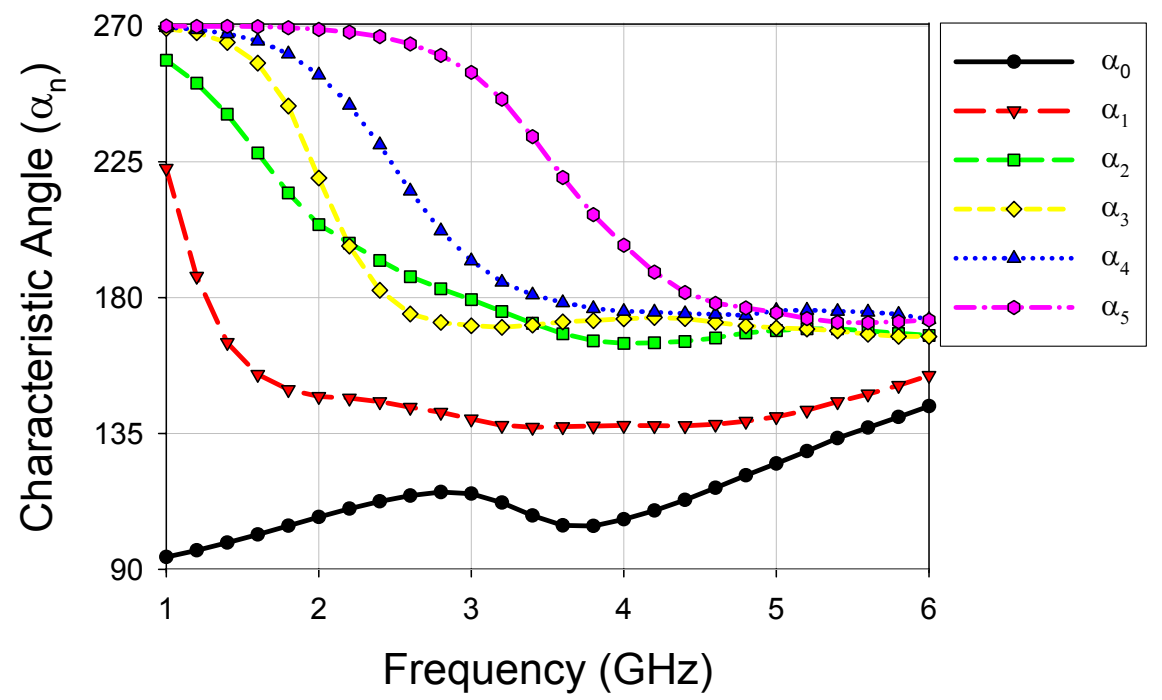

Figure 6.22 Variation with frequency of the characteristic angle associated to the current modes of Figure 6.21.

\begin{tabular}{|l|c|}
\cline { 2 - 2 } \multicolumn{1}{c|}{} & $f_{\text {res }}$ \\
\hline Mode $J_{1}$ & $1.20 \mathrm{GHz}$ \\
\hline Mode $J_{2}$ & $2.90 \mathrm{GHz}$ \\
\hline Mode $J_{3}$ & $2.50 \mathrm{GHz}$ \\
\hline Mode $J_{4}$ & $3.45 \mathrm{GHz}$ \\
\hline Mode $J_{5}$ & $4.45 \mathrm{GHz}$ \\
\hline
\end{tabular}

Table 6.4. Resonant frequencies for the radiating modes of the radiating ground plane with a notch.

Next thing to do is to feed the notch, and check which modes are excited. Figure 6.23 shows the return loss obtained when the notch is fed with a voltage gap generator placed at a narrow vertical strip at $3.75 \mathrm{~cm}$ from the shorted end of the notch. Unluckily, the antenna is not well matched, and it exhibits poor impedance bandwidth. But let us try to explain this behaviour with characteristic modes.

Understandably, the vertical voltage difference created by the feeding strip will favour the appearance of transmission-line currents flowing around the notch. This means that modes that present this kind of currents around the notch, like modes $J_{0}, J_{1}, J_{3}$ and $J_{5}$, will couple to the excitation and will be excited.

Figure 6.24 presents the total power radiated by the notched plane, together with the power radiated by the excited modes. These results confirm our previous deduction, since only modes $J_{0}, J_{1}, J_{3}$ and $J_{5}$, are excited. 
The total power radiated by the notch exhibits three maxima which correspond to the three minima of the return loss at $1.2 \mathrm{GHz}, 2,25 \mathrm{GHz}$, and $3.25 \mathrm{GHz}$. Mode $J_{I}$ is responsible for the first maximum of the total radiated power at $1.2 \mathrm{GHz}$. The second maximum at $2.25 \mathrm{GHz}$ is due to the superposition of the power radiated by modes $J_{0}$ and $J_{3}$, to the power radiated by mode $J_{1}$. Finally, the highest maximum at $3.25 \mathrm{GHz}$ is mainly due to mode $J_{0}$, but with the collaboration of modes $J_{1}$ and $J_{5}$.
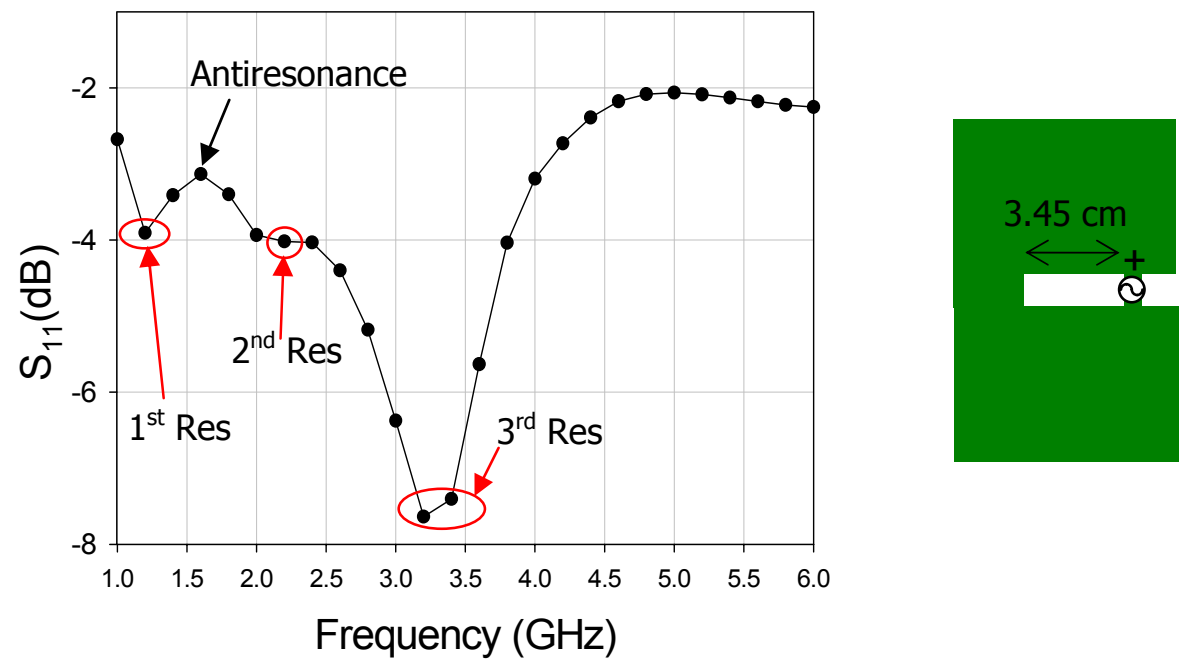

Figure 6.23 Return loss for $50 \Omega$, when the notch is fed with a voltage gap generator placed on a narrow metallic strip at $3.75 \mathrm{~cm}$ from the shorted end of the notch.

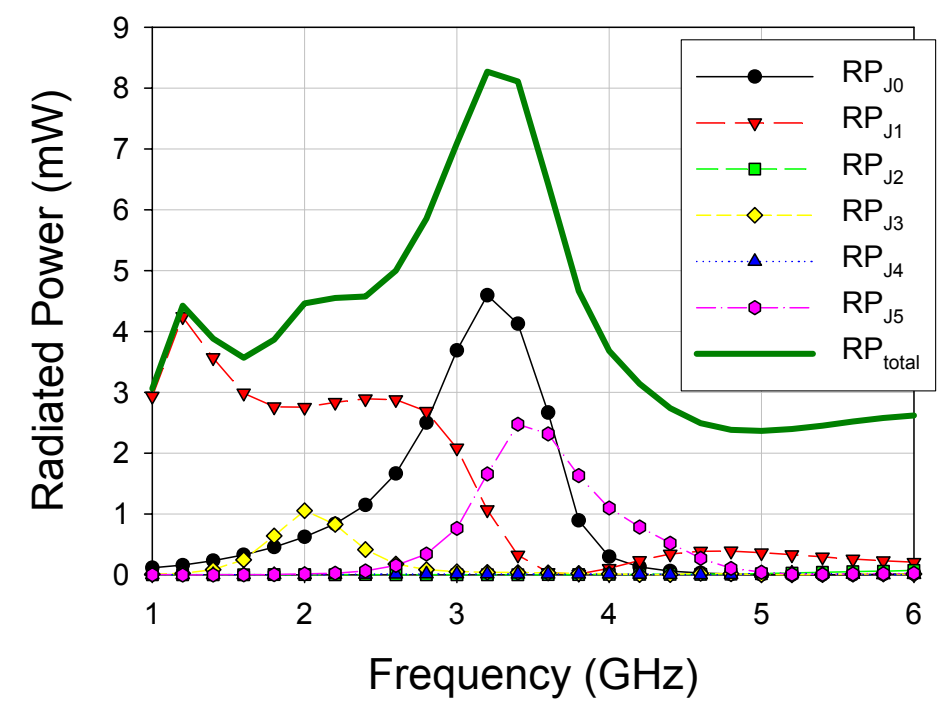

Figure 6.24 Contribution of the different modes to the total power radiated by the notched radiating ground plane. 
Now that all modes excited on the antenna are known, let us justify why the antenna is not well matched by studying the total current distribution at key frequencies.

Figure 6.25 presents the total current computed with IE3D at $1.2 \mathrm{GHz}$ (first resonance), $1.6 \mathrm{GHz}$ (antiresonance) and $3.25 \mathrm{GHz}$ (second resonance), when the notch is fed as described before. It can be observed that at $1.2 \mathrm{GHz}$ and 1.6 $\mathrm{GHz}$ there are intense currents forming a closed loop around the notch. Thus, it can be derived that when the notch is fed, the excitation of modes $J_{0,}, J_{1}, J_{3}$ and $J_{5}$, creates transmission-line currents around the notch that cause its resonance.

The notch resonates at $1.6 \mathrm{GHz}$, when its length $L_{1}$ is approximately $\lambda / 4$. This resonance of the notch interacts with the ground plane resonances at $1.2 \mathrm{GHz}$ and $2.4 \mathrm{GHz}$, associated to modes $J_{1}$ and $J_{3}$, respectively. The result is an antiresonance that causes the filtering effect at $1.6 \mathrm{GHz}$. On the other hand, the resonance at $3.25 \mathrm{GHz}$ is due to the excitation of the higher order vertical mode $J_{5}$, which does not interact with the notch. This is why the third minimum of the return loss presents better matching than the other two.

Next section proposes a doubly notched radiating ground plane with two excitations, to reduce the undesired filtering effect caused by the notch resonance.

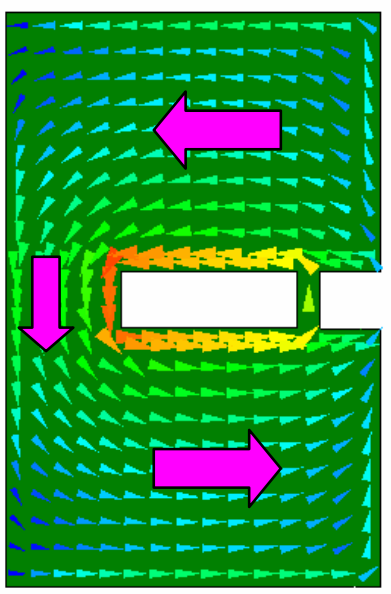

a) $1.2 \mathrm{GHz}$

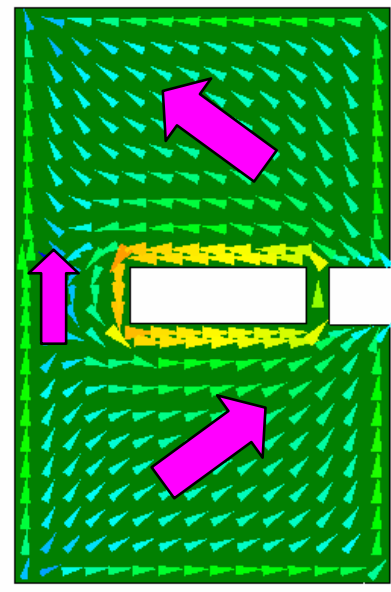

b) $1.6 \mathrm{GHz}$

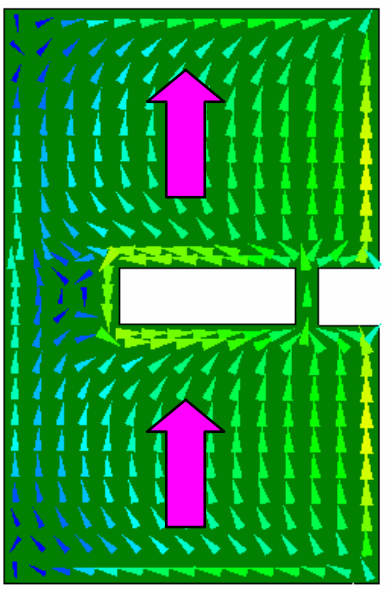

c) $3.25 \mathrm{GHz}$

Figure 6.25 Total current computed with IE3D at $1.2 \mathrm{GHz}, 1.6 \mathrm{GHz}$, and 3.2 $\mathrm{GHz}$ when the notch is fed as described in Figure 6.23. 


\subsubsection{Radiating ground plane with two notches.}

In order to improve the impedance matching of the radiating ground plane above described, a double-notch configuration is proposed. The ground plane is of exactly the same dimension of the one employed in section 6.3.1. The length of the notches is $L_{l}=4.7 \mathrm{~cm}$, and its width is $W_{l}=0.65 \mathrm{~cm}$.

Figure 6.26 illustrates the normalized current distribution at $1.1 \mathrm{GHz}$ for the first six characteristic modes of this ground plane with two notches. These modes resemble very much those obtained in Figure 6.21 for the ground plane with one notch, but now modes $J_{0,} J_{1}, J_{3}$ and $J_{4}$ are the modes that present transmission-line currents flowing along the notches. Observe that in modes $J_{2}$ and $J_{5}$ currents flow in phase in the upper and lower notches.

Figure 6.27 plots the variation with frequency of the characteristic angle associated to the modes of Figure 6.26. The bandwidth of these modes is not very different from that of the modes of the singly notched ground plane, with the exception of mode $J_{4}$, which now resonates at a lower frequency and presents poorer radiating bandwidth than in the singly notched ground plane.

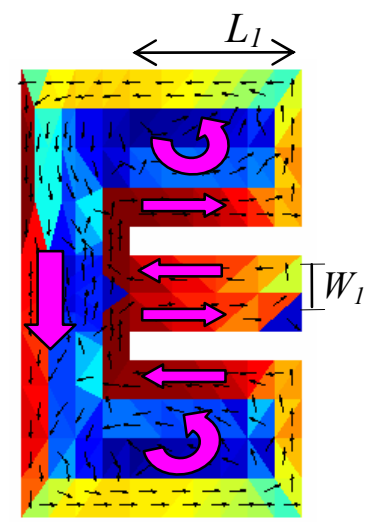

a) $J_{0}$

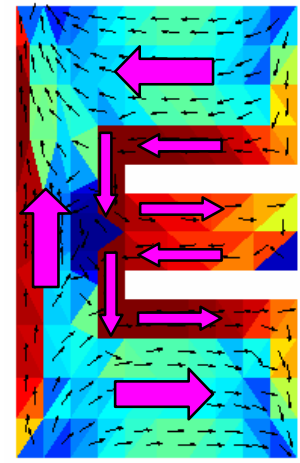

d) $J_{3}$

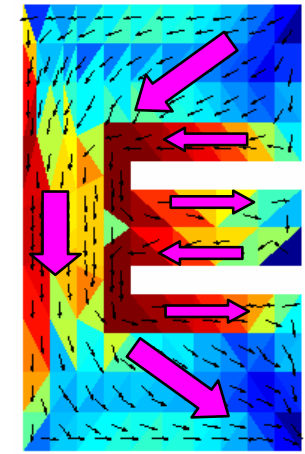

b) $J_{1}$

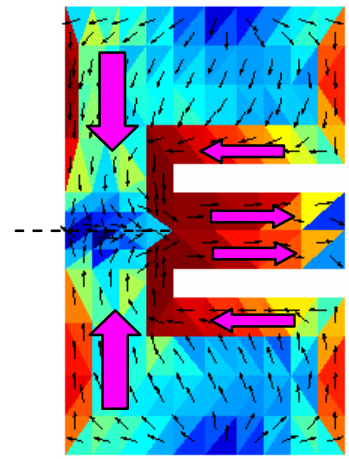

e) $J_{4}$

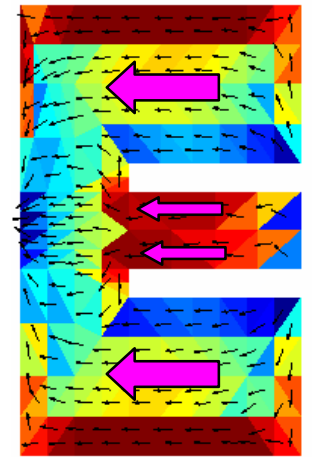

c) $J_{2}$

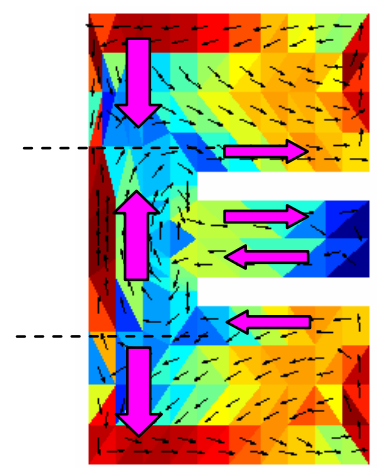

f) $J_{5}$

Figure 6.26 Normalized current distribution at $1.1 \mathrm{GHz}$ for the first six modes of the ground plane with two notches. 
The different behaviour of mode $J_{4}$ can be explained by examining its current distribution. In Figure 6.21 mode $J_{4}$ presents pure vertical currents and no current flow along the notch. In contrast, in Figure 6.26 mode $J_{4}$ has transverse current components that flow in a transmission line way along both the upper and lower notches. These current components are responsible for the reduction in the resonant frequency of the mode, and for the bandwidth degradation.

Table 6.5 summarizes the resonant frequencies of the radiating modes of the doubly notched ground plane. All these frequencies are smaller than those in Table 6.4 , because the two notches produce a longer current meandering than the single notch.

Regarding the impedance bandwidth that can be achieved from this structure, it is a well-known fact that when this E-shaped plane is mounted as a microstrip patch, and fed by a coaxial probe at its centre, it provides wideband performance [8]. Figure 6.28 depicts the return loss when an alternative feeding configuration is used. This new feeding consists in two voltage gap generators placed at $3.8 \mathrm{~cm}$ from the shorted ends of the notches.

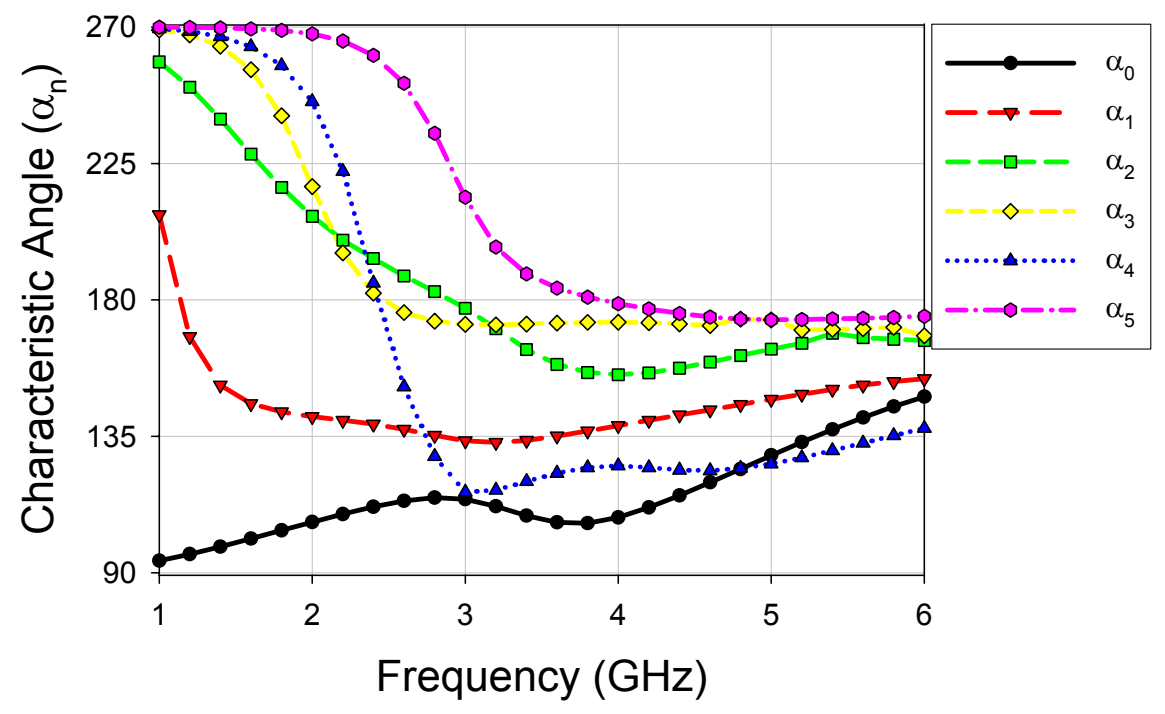

Figure 6.27 Variation with frequency of the characteristic angle associated to the current modes of Figure 6.26.

\begin{tabular}{|c|c|}
\cline { 2 - 2 } \multicolumn{1}{c|}{} & $f_{\text {res }}$ \\
\hline Mode $J_{1}$ & $1.10 \mathrm{GHz}$ \\
\hline Mode $J_{2}$ & $2.85 \mathrm{GHz}$ \\
\hline Mode $J_{3}$ & $2.45 \mathrm{GHz}$ \\
\hline Mode $J_{4}$ & $2.42 \mathrm{GHz}$ \\
\hline Mode $J_{5}$ & $3.9 \mathrm{GHz}$ \\
\hline
\end{tabular}

Table 6.5. Resonant frequencies for the radiating modes of the doubly notched radiating ground plane. 
The return loss of Figure 6.28 has been computed from the total input impedance obtained as the sum of the individual input impedances at each port, divided by two (parallel impedances). This return loss is much better than the one obtained for the singly notched ground plane. The second and third resonances are now at lowest frequencies, and the filtering effect at $1.6 \mathrm{GHz}$ has been attenuated.

Figure 6.29 shows the total current computed with IE3D at $1 \mathrm{GHz}, 2 \mathrm{GHz}$ and 3 $\mathrm{GHz}$, using this double excitation. On one hand, it is observed that the current distribution of mode $J_{l}$ is dominant at $1 \mathrm{GHz}$. Due to the feed symmetry, at $1 \mathrm{GHz}$ the current flowing on the centre of the plane is very weak. Moreover, because of the proximity of the two notches, there would be current cancellation between them. On the other hand, at $2 \mathrm{GHz}$ and $3 \mathrm{GHz}$ there is intense current flow on the feeding strips, so currents flowing on vertical direction are favoured, while transmission-line currents are minimized. Consequently, using two feed points the filtering effect due to the antiresonance is reduced, and the matching is improved.

Nevertheless, neither the feeding used for the single notch nor the double feeding just described, are realistic feedings. For the case of the doubly notched ground plane, a possible practical implementation of the feeding configuration could be that presented in Figure 6.30, where the two notches are fed by a microstrip power divider printed in the back side of the substrate. The substrate height is $7.6 \mathrm{~mm}$ and its permittivity 3.2. The length of the microstrip lines has been designed so that the two notches are fed in phase. Figure 6.31 shows two photographs of a prototype of this antenna fabricated at the laboratories of the GRE in UPV.

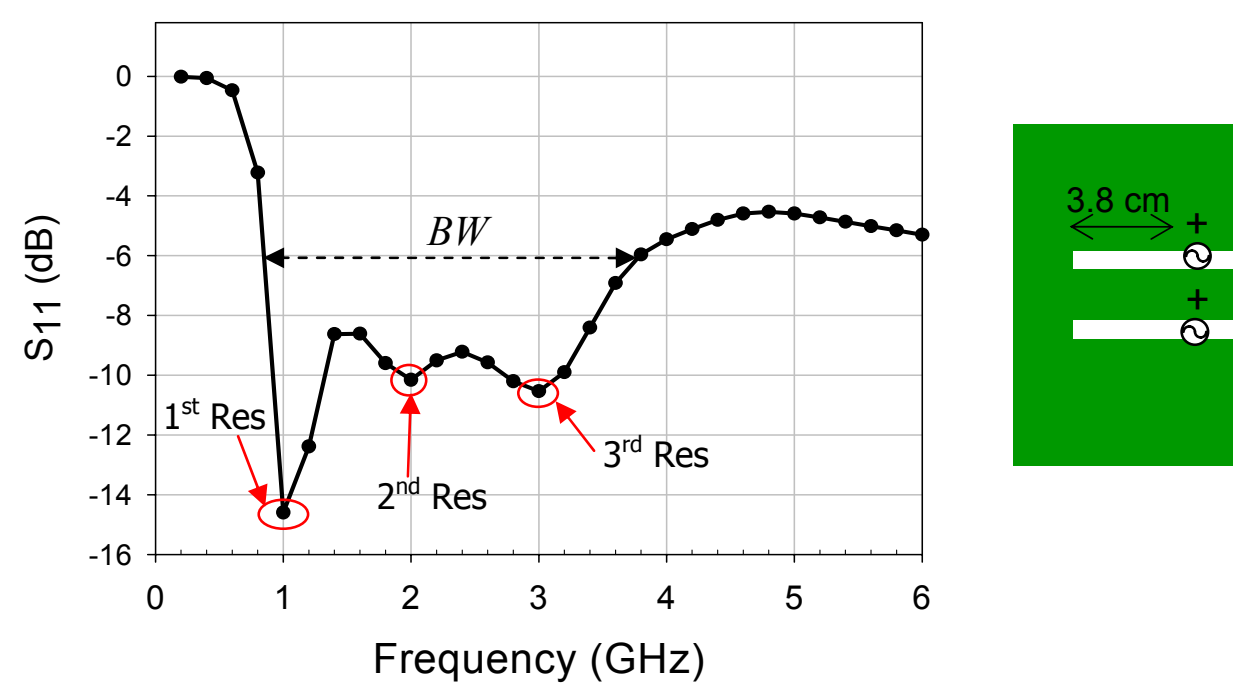

Figure 6.28 Return loss for $50 \Omega$, when the two notches are fed with voltage gap generators placed on narrow metallic strips at $3.8 \mathrm{~cm}$ from the shorted ends of the notches. 


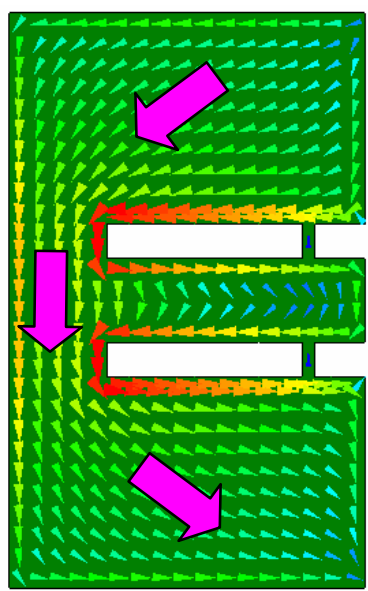

a) $1 \mathrm{GHz}$

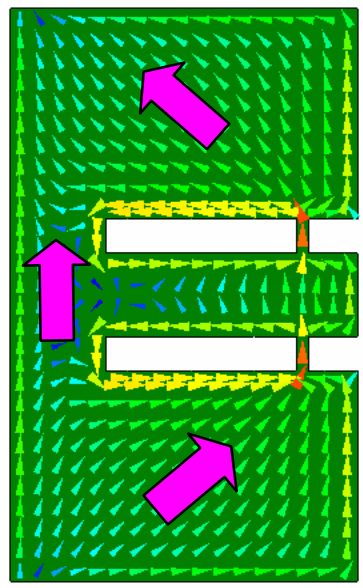

b) $2 \mathrm{GHz}$

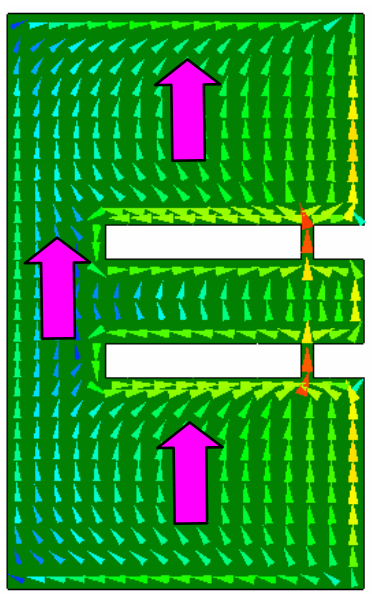

c) $3 \mathrm{GHz}$

Figure 6.29 Total current computed with IE3D at $1 \mathrm{GHz}, 2 \mathrm{GHz}$, and $3 \mathrm{GHz}$ when the notches are fed as described in Figure 6.28.

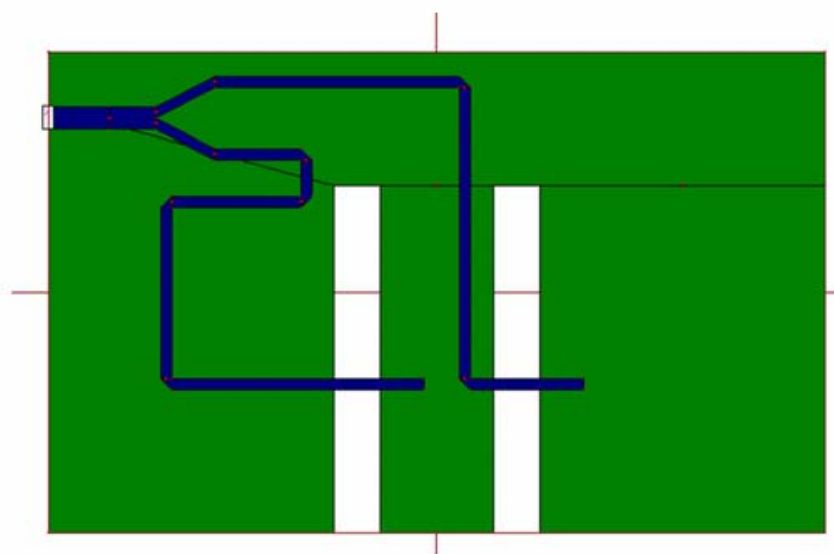

Figure 6.30 Possible feeding configuration for the doubly notched radiating ground plane.
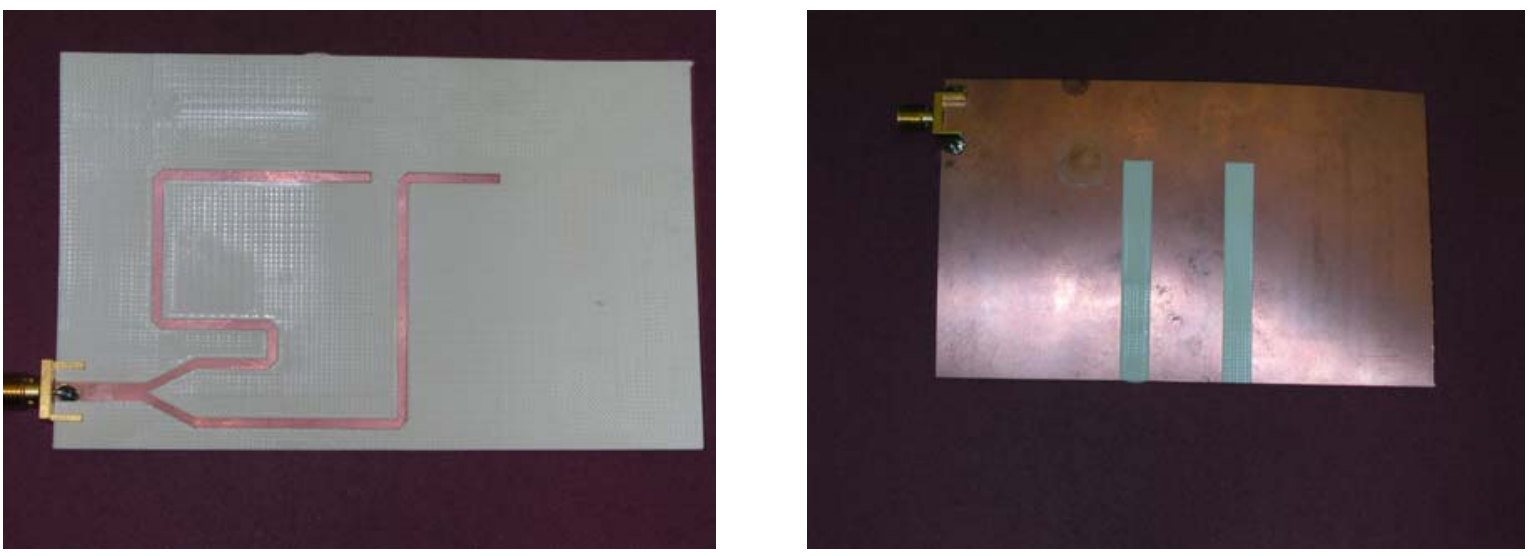

Figure 6.31 Photographs of the prototype for the doubly notched ground plane. 
Figure 6.32 depicts the return loss obtained from the IE3D simulations and the measurement. As observed, acceptable agreement exist between them, and the antenna presents a matched bandwidth for a return loss less than $-6 \mathrm{~dB}$ that extends from $1.6 \mathrm{GHz}$ to more than $5 \mathrm{GHz}$. A very wide bandwidth is hence obtained for this structure using this new feeding configuration.

Finally, it should be highlighted that, although the feeding configuration proposed in Figure 6.30 excites both notches simultaneously, it is not completely equivalent to that of the two voltage gap generators represented in Figure 6.28, because the two narrow metallic strips that support the feedings in the second case allow the current to flow though them, favouring in this way the excitation of vertical currents in the ground plane.

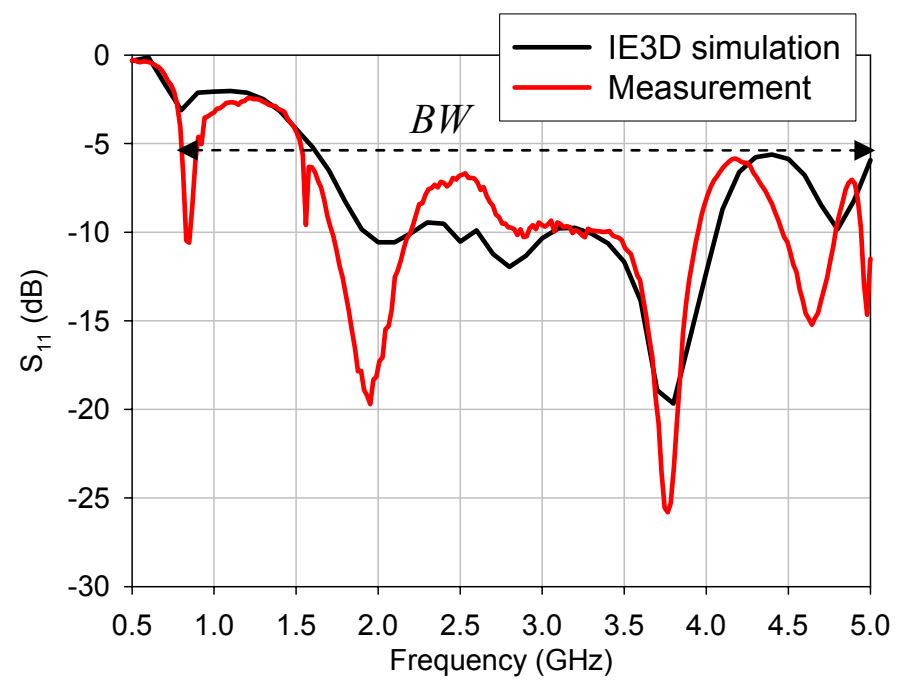

Figure 6.32 Simulated and measured return loss for the antenna prototype in Figure 6.31. 
CHAPTER 7. Conclusions and Future Work

\subsection{Conclusions}

\subsubsection{Suitability of characteristic modes for antenna design.}

The main goal of this thesis has been to demonstrate that the classical Theory of Characteristic Modes can be systematically applied to the design of wire and planar antennas.

Characteristic modes can be computed numerically for arbitrarily shaped conducting bodies. Since they form a set of orthogonal functions, they can be used to expand the total current on the surface of the body. However, what makes characteristic modes really attractive for antenna design is the physical insight they bring into the radiating phenomena taking place in the antenna.

As explained in Chapter 1, there exist alternative modal theories to analyze open radiating structures. However, the advantage of the Theory of Characteristic Modes with respect to other modal formalisms is the real nature of the eigenvectors and eigenvalues it supplies [30]. Especially important is the real nature of the eigenvalues that allows a physical interpretation of the radiating mechanisms of the antenna. As it has been demonstrated, information about the resonant frequency and radiating characteristics of modes can be obtained by studying the variation with frequency of the eigenvalues. Additionally, to compare the radiating behaviour of modes, a modal quality factor can be derived from the eigenvalues [38]. However, as it has been demonstrated in this work, this modal quality factor is only valid for narrow band radiators.

Moreover, the knowledge of the current distribution of the real eigenvectors is also very helpful for antenna design. A careful observation of the current distribution of the different modes brings information about its radiation efficiency and polarization purity. Also, by identifying the location of the current maxima, and taking into account the direction of the current flow, it is possible to find out an optimum feeding arrangement to excite the desired mode or modes. In general, modes in which currents flow in opposite phase, like in a transmission line, are not very efficient radiators, whereas modes with currents flowing in phase are good radiators in a wide range of frequencies.

Since characteristic modes are computed in the absence of any source or excitation, characteristic modes only depend on the shape and size of the radiating object. Thus, as demonstrated though numerous examples, antenna design using characteristic modes can be performed systematically in two steps. Firstly, the shape and size of the radiating element are optimized. Next, an optimum feeding configuration is chosen so that the desired modes may be excited. 
For modelling electrically small conducting bodies few modes are needed. Hence, small and intermediate size antennas can be fully characterized in a wide operating band just considering three or four characteristic modes.

An original contribution of this thesis is the determination of three different ways to identify which modes are excited for a particular excitation. First option consists in analyzing the contribution of modal input admittances to the total input admittance. Using this kind of representation it is possible to identify which modes are responsible for each resonance, and also which modes combine with each other to create an antiresonance. Nevertheless, modal admittances give no information about the matching of modes.

With the aim of determining which modes are well coupled to the source a modal VSWR can be computed from the modal input admittance. The main drawback of this representation is that the total VSWR can not be expressed as a linear expansion of the VSWR of the excited modes. However, it allows the identification of broadband matched modes, such as the modes that appear on monopoles with bevelled base.

The third and last option is the visualization of the modal radiated power. In this case, the total power of the antenna can be expanded very accurately in terms of power radiated by modes. With this representation it is possible to identify which modes produce the radiated power maxima at resonant frequencies.

It must be highlighted that many of the examples that have been presented in the first chapters, are not new designs, but well-known antennas whose behaviour is described in classical books of antenna theory. These examples have been chosen in order to initiate the reader into the Theory of Characteristic Modes by means of familiar antennas. For example, in Chapter 2 the basic formulation of the theory has been reviewed using a wire dipole as example, in order to facilitate the comprehension of the fundamentals of the theory. In Chapter 3 loop antennas of different geometry have been analyzed from a modal point of view. The objective of Chapter 3 was to make the reader realize that the Theory of Characteristic Modes complements classical antenna theory, bringing a physical explanation of the radiating mechanisms of the antenna. Actually, examples presented in the first chapters are perfect to provide novel antenna designers with a physical understanding of the radiation of antennas.

In the first part of Chapter 5, it has been demonstrated that characteristic modes constitute an alternative to analyze patch antennas when the cavity model fails. In contrast with the cavity model, characteristic modes present no limitation over the shape of the patch, the thickness, or the dielectric constant of the substrate. 
Moreover, also in Chapter 5 characteristic modes have been used to adjust the phase of the field reflected by the individual elements of a reflectarray antenna. An expression for the reflection phase of each mode has been derived from the eigenvalues, and it has been shown that for the case of oblique incidence, the polarization purity of a square patch can be improved just by dividing the patch in two rectangular strips along the direction of the desired polarization.

The second part of Chapter 5 has been intended to give some guidelines for the design of planar monopoles. This part shows how a clear knowledge of the basic operating principles of very simple monopoles has lead to the design of new antennas, such as the shorted arrowhead monopole, the double fed square monopole, and the slitted monopole for MIMO applications.

Chapter 6 describes the procedure carried out to design several handset antennas, based on the PCB resonance concept, using characteristic modes. In these designs, wideband impedance matching is achieved by a proper choice of the feeding configuration. For the case of the folded radiating ground plane, using characteristic modes it has been demonstrated that a planar feeding monopole provides better performance than a classical coaxial feeding probe. In contrast, for the case of the radiating ground plane with two notches, a double fed has been found to be the optimum choice.

\subsubsection{Suitability of characteristic modes for numerical analysis.}

Chapter 4 has been focused on analyzing the problematic derived from using characteristic modes as basis functions in which to expand the total current on the antenna. By means of different examples, it has been demonstrated that for antenna problems, the series of characteristic modes presents slow convergence when approximating the imaginary part of the input current. As a contribution of this thesis, the convergence problem of the series of modes has been overcome by including a new term in the modal expansion, called "source mode". This source mode exhibits evanescent behaviour and models the reactive behaviour introduced by the source. Guidelines have been given in order to systematically obtain the source mode for both the wire antenna class and planar antennas.

On the other hand, the dependency with frequency of characteristic modes has also been solved by using a Singular Value Decomposition (SVD) [47]. The Singular Value Decomposition provides a set of frequency independent modes that can be used as basis functions in the whole band of interest. If the source mode in included in the SVD, a set of frequency independent characteristic modes that fastens the convergence of the series in a wide range of frequencies is obtained. Note that although the SVD approach is not new [89], the inclusion of the source mode in the decomposition in order to solve the convergence problem of characteristic modes has been proposed in this thesis for the first time. 


\subsection{Future work}

One of the most appealing aspects of the Theory of Characteristic Modes is the possibility of using characteristic modes in conjunction with reactive loading for the control of radiation and scattering of conducting bodies [38]-[39]. As explained in Chapter 2, when reactive loading is combined with characteristic modes it gains a more practical perspective since total control of the electromagnetic behaviour of the antenna can be achieved. In fact, using reactive loading a mode can be made resonant at any desired frequency. In this work only the effect of reactive loading on wire antennas has been analyzed. However, further research could be made in order to include reactive loading in planar structures. This loading could be discrete or distributed. An example of distributed load could consist in a slot. The effect of the slot could be modelled by a series of discrete loads placed at different edge elements. Using a discrete model, slots of different length and at different positions, could be simulated without creating a new structure, just changing the value of the discrete loads.

Another option for future work could consist in making the design procedure even more systematic. This could be accomplished by combining the information yielded by characteristic modes with a genetic algorithm, or another automatic optimization technique, in order to find the optimum location for the feeding of the antenna. Note that although characteristic modes help to identify the current maxima of modes, these maxima may not be exactly the optimum feeding location. In practice, the optimum position of the feeding is obtained by "cut and try" methods. Hence, characteristic currents could be used to obtain a coarse approximation of the optimum location for the feeding, while a genetic algorithm would perform a refine search.

On the other hand, in Chapter 4 it has been commented that the SVD approach to obtain a set of frequency independent characteristic modes is worthwhile only when the antenna is to be analyzed is a wide range of frequencies, and also that this approach is especially suitable to reduce the computational cost when analyzing large arrays with identical wideband radiating elements. Nevertheless, no experiment has been performed in order to compare the time requirements to solve a large array problem using classical MoM formulation, and using characteristic modes as entire domain basis function. Thus, future work may verify that the use of characteristic modes in large array problems reduces computational time.

Finally, it should be mentioned that Eva Antonino is developing a thesis related with Characteristic Modes that complements the present work. Basically, this second thesis concentrates on analyzing the effect of using multiple feeding ports, and on modelling the feeding gap zone of square planar monopoles using a transmission line theory approach. 


\section{$\underline{\text { References }}$}

[1] K. Hirasawa, M. Haneishi, et al., "Analysis, Design and Measurement of Small and Low-profile Antennas," Boston, London: Artech House Antennas and Propagation Library, 1992.

[2] K. Fujimoto, et al., "Small Antennas," England, Research Studies Press, 1993.

[3] K. L. Wong, "Compact and Broadband Microstrip Antennas," Wiley-Interscience, John Wiley \& Sons, 2002.

[4] D. B. Miron, "Small Antenna Design," Communications Engineering Series, Newnes, April 2006.

[5] J. S. McLean, "A Re-Examination of the Fundamental Limits on the Radiation Q of Electrically Small Antennas," IEEE Transactions on Antennas and Propagation, AP-44, May 1996, pp. 672-676.

[6] H. Morishita, Y. Kim, and K. Fujimoto, "Design Concept of Antennas for Small Mobile Terminals and the Future Perspective," IEEE Trans. Antennas Propagat. Magazine, vol.44, no. 5, Oct. 2002, pp. 30-43.

[7] K. L. Wong, "Planar Antennas for Wireless Communications," Wiley-Interscience, John Wiley \& Sons, 2000.

[8] A. F. Peterson, S. L. Ray, and R. Mittra, "Computational Methods for Electromagnetics," IEEE Press Series on Electromagnetic Wave Theory, WileyIEEE Press, 1997.

[9] E. K. Miller, L. Medgyesi-Mitschang, and E. H. Newman, eds., "Computational Electromagnetics: Frequency-Domain Method of Moments", IEEE Press, 1992.

[10] W. C. Chew, J. Jin, E. Michielssen, J. Song, eds, "Fast and Efficient Algorithms in Computational Electromagnetics," Artech House 2001.

[11] "IE3D User's Manual," Release 10. Zeland Sofware Inc., June 2003.

[12] "FEKO User's Manual," Suite 5.1, EM Software \& Systems, South Africa, December 2005.

[13] "User Manual for the 3D EM Time Domain Simulator Empire," IMST GmbH, Germany, January 2004.

[14] "User Manual for HFSS," Ansoft Corporation, Pittsburg, PA, USA.

[15] E. K. P. Chong, S. H. Żak, "An Introduction to Optimization," Wiley-Interscience Series in Discrete Mathematics and Optimization. John Wiley \& Sons, New York, 2001.

[16] Y. Rahmat-Samii, E. Michielssen, "Electromagnetic Optimization by Genetic Algorithms," New York: John Wiley \& Sons, 1999.

[17] D. S. Linden, "Automated Design and Optimization of Wire Antennas Using Genetic Algorithms," Ph. D. Dissertation, Department of Electrical Engineering and Computer Science, Sept.1997

[18] C. Christodoulou, M. Georgiopoulos, C. Christopoulos, "Applications of Neural Networks in Electromagnetics," Artech House, 2001.

[19] C. G. Christodoulou, M. Georgioipoulos and A.H. El Zooghby, "Applications of Neural Networks in Smart Antennas for Mobile Communications," Applied Computational Intelligence, CRC Press, LLC, Jan. 2000.

[20] J. Robinson and Y. Rahmat-Samii, "Particle Swarm Optimization in Electromagnetics," IEEE Trans. Antennas Propagat., vol. 52, Iss. 2, Feb. 2004, pp. $397-407$.

[21] D. T. Pham, et al. "The Bees Algorithm: A Novel Tool for Complex Optimisation Problems," Proc. $2^{\text {nd }}$ Int. Virtual Conf. on Intelligent Production Machines and Systems (IPROMS 2006), Oxford, Elsevier, 2006. 
[22] R. E. Collin, "Field Theory of Guided Waves," McGraw Hill, New York, 1960.

[23] R. Mittra and S. W. Lee, "Analytical Techniques in the Theory of Guided Waves," Macmillan, New York, 1971.

[24] L. J. Chu, "Physical Limitations of Omnidirectional Antennas," J. Appl. Phys., vol. 19, pp. 1163-1175, December 1948.

[25] R. F. Harrington, "Effect of Antenna Size on Gain, Bandwidth and Efficiency," J. Res. Nat. Bur. Stand.-D, Radio Propagation, vol. 64D, pp. 1-12, Jan-Feb. 1960.

[26] R. G. Parkinson and M. M. Z. Kharadly, "Optimum Determination of Scatteredfield Coefficients from Near-field Measurements," Proceedings of the IEEE, vol. 58, no. 9, pp. 1396-1397, Sept. 1970.

[27] D. Wilton and R. Mittra, "A New Numerical Approach to the Calculation of Electromagnetic Scattering Properties of Two-dimensional Bodies of Arbitrary Cross Section," IEEE Trans. Antennas Propagat., vol. 20, no. 2, pp. 310-317, May 1972.

[28] R. J. Garbacz, "A Generalized Expansion for Radiated and Scattered Fields," Ph.

D. dissertation, Ohio State University, Columbus, 1968.

[29] R. J. Garbacz and R. H. Turpin, "A Generalized Expansion for Radiated and Scattered Fields," IEEE Trans. Antennas Propagat., vol. AP-19, May 1971, pp. 348-358.

[30] R. F. Harrington and J. R. Mautz, "Theory of Characteristic Modes for Conducting Bodies," IEEE Trans. Antennas Propagat., vol. AP-19, no. 5, pp. 622628, Sept. 1971.

[31] R. F. Harrington and J. R. Mautz, "Computation of Characteristic Modes for Conducting Bodies," IEEE Trans. Antennas Propagat., vol. AP-19, no. 5, pp. 629639, Sept. 1971.

[32] R. F. Harrington, J. R. Mautz, and Y. Chang, "Characteristic Modes for Dielectric and Magnetic Bodies," IEEE Trans. Antennas Propagat., vol. AP-20, pp. 194-198, March 1972.

[33] N. Inagaki, "Eigenfunctions of Hermitian Iterated Operator and its Application to Numerical Analysis," Dig. Int. Symp. Antennas Propagat., Japan, pp. 295-298, Sept. 1978.

[34] N. Inagaki and R. J. Garbacz, "Eigenfunctions of Composite Hermitian Operators with Application to Discrete and Continuous Radiating Systems," IEEE Trans. Antennas Propagat., vol. AP-30, pp.571-575, July 1982.

[35] D. M. Pozar, "Antenna Synthesis and Optimization Using Weighted Inagaki Modes," IEEE Trans. Antennas Propagat., vol. AP-32, no.2, pp. 159-165, Feb. 1984.

[36] D. Liu, R. J. Garbacz and D. M. Pozar, "Antenna Synthesis and Optimization Using Generalized Characteristic Modes," IEEE Trans. Antennas Propagat., vol. 38, no.6, pp. 862-868, June 1990.

[37] R. J. Garbacz and D. M. Pozar, "Antenna Shape Synthesis Using Characteristic Modes," IEEE Trans. Antennas Propagat., vol. 30, no. 3, pp. 340-350, May 1982.

[38] R. F. Harrington and J. R. Mautz, "Control of Radar Scattering by Reactive Loading," IEEE Trans. Antennas Propagat., vol. AP-20, no. 4, pp. 446-454, July 1972.

[39] R. F. Harrington and J. R. Mautz, "Pattern Synthesis for Loaded N-port Scatterers," IEEE Trans. Antennas Propagat., vol. AP-22, no. 2, pp. 184-190, March 1974.

[40] J. R. Mautz and R. F. Harrington, "Radiation and Scattering from Bodies of Revolution," Appl. Sci. Res., vol.20, pp.405-435, June 1969. 
[41] B. A. Austin and K. P. Murray, "The Application of Characteristic-Mode Techniques to Vehicle-Mounted NVIS Antennas," IEEE Trans. Antennas Propagat., vol. 40, no. 1, pp. 7-21, Feb. 1998.

[42] R. F. Harrington, "Field Computation by Moment Method," Macmillan, New York, 1968.

[43] "Using Matlab" The Math Works Inc., 1999.

[44] S. M. Rao, D. R. Wilton and A. W. Glisson, "Electromagnetic Scattering by Surfaces of Arbitrary Shape," IEEE Trans. Antennas and Propagat. AP-30, 1982, pp. 409-418.

[45] S. N. Makarov, "Antenna and EM Modeling with Matlab," Wiley-Interscience, John Wiley \& Sons, New York, July 2002.

[46] E. H. Newman, "Generation of Wide-band Data from the Method of Moments by Interpolating the Impedance Matrix," IEEE Trans. Antennas Propagat., vol. 36, no. 12 , pp. 1820-1824, Dec. 1998.

[47] Z. Bai, J. Demmel, J. Dongarra, A. Ruhe and H. Van Der Vorst, eds., "Templates for the Solution of Algebraic Eigenvalue Problems: A Practical Guide," SIAM (Society for Industrial \& Applied Math), Philadelphia, 2000.

[48] E. Suter, and J. R. Mosig, "A Subdomain Multilevel Approach for the Efficient MoM Analysis of Large Planar Antennas," Microwave and Optical Technology Letters, vol. 26, no. 4, August 2000, pp. 270-277.

[49] W. Geyi, P. Jarmuszewski, and Y. Qi, "The Foster Reactance Theorem for Antennas and Radiation Q," IEEE Trans. on Antennas and Propagation, vol. 3, no. 3, pp. 401-408, March 2000.

[50] S. R. Best, "The Foster Reactance Theorem and Quality Factor for Antennas," IEEE Antennas and Wireless Propagation Letters, vol. 3, pp. 306-309, 2004.

[51] E. H. Newman, "Small antenna Location Synthesis Using Characteristic Modes," IEEE Trans. Antennas Propagat., vol. AP-21, no. 6, pp. 868-871, Nov. 1973.

[52] D. Seth, and Y. Chow, "On Linear Parasitic Array of Dipoles with Reactive Loading," IEEE Trans. on Antennas and Propagation, vol. 21, no. 3, pp. 286292, May 1973.

[53] R. L. Li, V. F. Fusco, and R. Cahill, "Pattern Shaping Using a Reactively Loaded Wire Loop Antenna," IEE Proc.- Microw. Antennas Propagat., vol. 148, no.3, June 2001.

[54] H. Scott, and V. F. Fusco, "Electronically Beam Tilting Using a Single Reactively Loaded Circular Wire Loop Antenna," IEE Proc. Microwaves, Antennas and Propagation, vol. 149, no. 56, pp. 271-274, Oct.-Nov. 2002.

[55] W. Richards, S. Davidson, and S. Long, "Dual-band Reactivaly Loaded Microstrip Antenna," IEEE Trans. on Antennas and Propagation, vol. 33, no. 5, pp. 556561, May 1985.

[56] A. Ali-Khan, W. F. Richards, and S. A. Long, "Impedance Control of Microstrip Antennas Using Reactive Loading," IEEE Trans. on Antennas and Propagation, vol. 37, no. 2, pp. 247-251, Feb. 1989.

[57] S. Maci, G. Biffi Gentili, P. Piazzesi, and C. Salvador, "Dual-band Slot-loaded Patch Antennas," IEE Proc. H. Microwave Antennas Propagat., 142, pp. 225232, 1995.

[58] K. Yegin, and A. Q. Martin, "Very Broadband Loaded Monopole Antennas," Antennas and Propagat. Society Int. Symposium, vol. 1, pp. 232-235, July 1997.

[59] S. Seong-Youp, W. L. Stutzman, and W. A. Davis, "Multi-broadband Monopole Disc Antennas," Antennas and Propagat. Society Int. Symposium, vol. 3, pp. 616-619, June 2003.

[60] R. F. Harrington, "Theory of Loaded Scatterers," Proc. IEE, 111, pp. 617-623, 1964. 
[61] R. F. Harrington, and J. L. Ryerson, "Electromagnetic Scattering by Loaded Wire Loops," Radio Sci., 1, pp. 347-352, 1966.

[62] R. F. Harrington, and J. R. Mautz, "Straight Wires with Arbitrary Excitation and Loading," IEEE Trans. on Antennas and Propagat., vol. AP-15, pp. 502-515, 1967.

[63] K. R. Carver, and W. M. James, "Microstrip Antenna Technology," IEEE Trans. on Antennas and Propagation, vol. AP-29, no. 1, pp. 2-22, January 1981.

[64] G. K. H. Liu, and R. D. Murch, "Compact Dual-Frequency PIFA Design Using LC Resonators," IEEE Trans. on Antennas and Propagation, vol. 50, no. 10, pp. 1433-1444, Oct. 2002.

[65] P. Vainikainen, J. Ollikainen, O. Kivekäs and I. Kelander, "Resonator-Based Analysis of the Combination of Mobile Handset Antenna and Chassis," IEEE Trans. Antennas Propagation., vol.50, no. 10, pp. 1433-1444, Oct. 2002.

[66] Shivnaravan, and B. R. Visvakarma, "Analysis of Dual-band Patch Antenna for Mobile Communications," Microwave and Opt. Tech. Letters, vol.47, no. 6, pp. 558-564, Dec. 2005

[67] G. W. Streable, and L. W. Pearson, "A Numerical Study on Realizable BroadBand and Equivalent Admittances for Dipole and Loop Antennas," IEEE Trans. on Antennas and Propagation, Vol. AP-29, No.5, September 1981.

[68] T. G. Tang, Q. M. Tieng, and M. W. Gunn, "Equivalent Circuit of a Dipole Antenna Using Frequency-Independent Lumped Elements," IEEE Trans. on Antennas and Propagation, vol. 41, no.1, pp. 100-103, January 1993.

[69] M. Hamid and R. Hamid, "Equivalent Circuit of Dipole Antenna of Arbitrary Length," IEEE Trans. on Antennas and Propagation, vol. 45, no.11, pp. 16951696, November 1997.

[70] B. R. Long, P. L. Werner, D. H. Werner, "Genetic-algorithm Optimization of Dipole Equivalent Circuit Models," Microwave and Optical Technology Letters, vol. 27, no. 4, pp. 259-261, Sept 2000.

[71] S. A. Schelkunoff, "Representation of Impedance Functions in Terms of Resonant Frequencies," Proc. IRE, vol. 32, pp. 83-90, Feb. 1944.

[72] C. E. Baum, "The Singularity Expansion Method," Transient Electromagnetic Fields, edited by L. B. Felsen, Springer-Verlag, Berlin, 1976.

[73] K. A. Michalski, and L. W. Pearson, "Equivalent Circuit Synthesis for a Loop Antenna Based on the Singularity Expansion Method," IEEE Trans. on Antennas and Propagation, vol. AP-32, no.5, pp. 433-441, May 1984.

[74] Y. Kim, H. Ling, "Equivalent Circuit Modelling of Broadband Antennas Using a Rational Function Approximation," Microwave and Optical Technology Letters, vol. 48, no. 5, pp. 950-953, March 2006.

[75] E. J. Rothwell, and N. Gharsallah, "Determination of the Natural Frequencies of a Thin Wire Elliptical Loop," IEEE Trans. Antennas and Propagat., vol. AP-35, pp. 1319-1324, 1987.

[76] T. H. Shumpert, L. S. Riggs, and J. M. Lindsey, "Singularity Expansion Method Analysis of Regular Poygonal Loops," IEEE Trans. Antennas Propag., vol. 38, no. 8, August 1990.

[77] D. Foster, "Loop Antenna with Uniform Current," Proc. IRE, vol. 32, pp. 603607; October 1944.

[78] J. E. Storer, "Impedance of Thin-Wire Loop Antennas," AIEE Trans., vol. 75, pp. 606-619, Nov. 1956.

[79] J. E. Lindsay, Jr. "A Circular Loop Antenna with Non-Uniform Current Distribution," IRE Trans. on Antennas and Propag., vol. 8, pp. 439-441, July 1960. 
[80] L. W. Li, C.P. Lim and M. S. Leong, "Method of Moments Analysis of Electrically Large Circular Loop Antennas: Non-uniform Currents," IEE ProceedingsMicrowaves, Antennas and Propagat., vol. 146, no. 6, pp. 416-420, Dec. 1999.

[81] RL Li, V. Fusco, and H. Nakano, "Circularly Polarized Open-Loop Antenna," IEEE Trans. Antennas Propag., vol. 51, no. 9, pp. 2475-2477, Sep. 2003.

[82] R. L. Li, N. A. Bushyager, J. Laskar, and M. M. Tentzeris, "Determination of Reactance Loading for Circularly Polarized Circular Loop Antennas with a Uniform Traveling-Wave Current Distribution" IEEE Trans. Antennas Propag., vol. 53, no. 12, Dec. 2005.

[83] T. Tsukiji, and S. Tou, "On Polygonal Loop Antennas," IEEE Trans. Antennas Propag., vol. AP-28, no. 4, July 1980.

[84] W. Choe, and J. K. Lee, "Analysis of Higher Order Regular Polygonal Loop Antennas," IEEE Trans. Antennas Propag., vol. 38, no. 7, July 1990.

[85] R. King, "Theory of the Corner-Driven Loop Antenna," IRE Trans. Antennas and Propagat., vol. AP-4, p.393, July 1956.

[86] C. A. Balanis, "Antenna Theory: Analysis and Design," John Wiley \& Sons, Nueva York, 1997.5

[87] C. E. Smith, C. M. Butler, and K. R. Umashankar, "Characteristics of a Wire Biconical Antenna," Microwave Journal, vol. 22, no. 8, pp. 37-40, Sept. 1979.

[88] G. Amendola, G. Angiulli, and G. Di Massa, "Numerical and Analytical Characteristic Modes for Conducting Elliptic Cylinders," Microwave and Opt. Tech. Letters, vol. 16, no. 4, Nov. 1997.

[89] V. V. S. Prakash, "RCS Computation over a Frequency Band using the Characteristic Basis and Model Order Reduction Method", Antennas and Propagat. Society Int. Symposium, vol. 4, pp. 89-92, June 2003.

[90] V. V. S. Prakash, and R. Mittra, "Characteristic Basis Function Method: A new Technique for Efficient Solution of Method of Moments Matrix Equations," Microwave and Opt. Technology Letters, vol. 36, no. 2, pp. 95-100, Jan. 2003.

[91] G. Vecchi, P. Pirinoli, L. Matekovits, and M. Orefice, "Use of Dynamic Modes in the Analysis of Printed Antennas and Arrays," IEEE Antennas and Propagat. Society Int. Symposium, vol.4, pp. 1834-1837, June 1998.

[92] M. Djordjevic and B. M. Notaros, "Three Types of Higher-order MoM Basis Functions Automatically Satisfying Current Continuity Conditions," Antennas and Propagat. Society Int. Symposium, vol. 4, pp. 610-613, 2002.

[93] J. M. Bornholdt, and L. N. Medgyesi-Mitschang, "Mixed-domain Galerkin Expansions in Scattering Problems," IEEE Trans. Antennas Propag., vol. 36, no. 2, pp. 216-227, Feb. 1988.

[94] G. Angiulli, and G. Di Massa, "Mutual Coupling Evaluation in Microstrip Arrays by Characteristic Modes," Electrotechnical Conference, 1996, MELECON 96, vol. 1, pp. 580-583, May 1996.

[95] G. Angiulli, and G. Di Massa, "Scattering from Arbitrarily Shaped Microstrip Patch Antennas Using the Theory of Characteristic Modes," Antennas and Propagat. Society Int. Symposium, vol. 4, pp. 1830-1833, June 1998.

[96] A. O. Yee, and R.J. Garbacz, "Self- and Mutual-admittances of Wire Antennas in Terms of Characteristic Modes," IEEE Trans. Antennas Propagat., vol. AP-21, no. 6, pp. 868-871, Nov. 1973.

[97] D. G. Dudley, "Mathematical Foundations for Electromagnetic Theory," IEEE Press Series on Electromagnetic Waves, 1994.

[98] Y. Hua, and T. K. Sarkar, "Generalized Pencil-of-Function Method for Extracting Poles of an EM System from its Transient Response," IEEE Trans. Antennas Propagat, vol.37, no. 2, pp.229-234, Feb. 1989. 
[99] J. R. James and P.S. Hall, "Handbook of Microstrip Antennas," IEE electromagnetic waves series. Peter Peregrinus Ltd., London, 1989.

[100] D. M. Pozar, and Daniel H. Schaubert, "Microstrip Antennas: The Analysis and Design of Microstrip Antennas and Arrays," New York, 1995.

[101] R. Garg, P. Bhartia, I. Bahl, and A. Ittipiboon, "Microstrip Antenna Design Handbook," Artech House antennas and propagation library, Artech House, 2001.

[102] N.P. Agrawall, G. Kumar, and K.P. Ray, "Wideband Planar Monopole Antennas," IEEE Trans. Antennas Propag., vol. 46, pp.294-295, February 1998.

[103] M. J. Ammann and Z. N. Chen, "Wideband Monopole Antennas for Multi-band Wireless Systems," IEEE Trans. Antennas Propagat. Magazine, vol.45, no. 2, pp. 146-150, April. 2003.

[104] H. Schantz, "The Art and Science of Ultrawideband Antennas," Artech House 2005.

[105] R. B. Waterhouse, "Stacked Patches Using High and Low Dielectric Constant Material Combinations," IEEE Trans, on Antennas and Propagat., vol. 47, no. 12, pp. 1767 - 1771, Dec. 1999.

[106] B. Ooi, S. Qin, and M. Leong, "Novel Design of broadband stacked patch antenna," IEEE Trans. Antennas Propagat, vol. 50, no. 10, pp. 1391-1395, Oct. 2002.

[107] W. S. T. Rowe, and R. B. Waterhouse, "Investigation into the Performance of Proximity Coupled Stacked Patches," IEEE Trans. Antennas Propagat, vol. 54, no. 6, pp. 1693-1698, June 2006.

[108] G. Kumar, and K. C. Gupta, "Directly Coupled Multiple Resonator Wide-band Microstrip Antennas," IEEE Trans. Antennas Propagat. Vol. 33, no. 6, pp. 588593, June 1985.

[109] R. Garg, and V. S. Reddy, "A Broad-band Coupled-strips Microstrip Antenna," IEEE Trans. on Antennas and Propagat., vol. 49, no. 9, Sept. 2001.

[110] S. V. Hum, J. Chu, R. H. Johnston, and M. Okoniewski, "Improving the Bandwidth of Microstrip Patch Antennas Using Resistive Loading," IEEE Antennas and Propagat. Society Int. Symposium, vol. 2, pp. 276-279, June 2003.

[111] S. V. Hum, J. Z. Chu, R. H. Johnston, and M. Okoniewski, "Efficiency of a Resistively Loaded Microstrip Patch antenna," Antennas and Wireless Propagation Letters, vol. 2, no. 1, pp. 22-25, 2003.

[112] Y. Suzuki, N. Miyano, T. Chiba, "Circularly Polarized Radiation from Singly fed Equilateral-triangular Microstrip Antenna," in Microstrip Antenna Design, K. C. Gumpta and A. Benalla (Eds.), Artech House, Norwood, MA, 1988, pp. 338-342.

[113] R. E. Munson, H. Haddad, and J. Hanlen, "Microstrip Reflectarray Antenna for Satellite Communication and RCS Enhancement or Reduction," U. S. Patent No. $4684952,1987$.

[114] T. A. Metzler, "Design and Analysis of a Microstrip Reflectarray," Ph. D. Dissertation, University of Massachusetts, Amherst, February 1993.

[115] TDA Progress Report 42-120, "Analysis of a microstrip reflectarray antenna for microspacecraft applications," February 1995.

[116] J. A. Encinar, J. A. Zornoza, "Broadband Design of Three-layer Printed Reflectarrays," IEEE Trans. Antennas Propagat. Vol. 51, no. 7, pp. 1662-1664, July 2003.

[117] M. Bozzi, S. Germani, L. Perregrini, "Performance Comparison of Different Element Shapes Used in Printed Reflectarrays," Antennas and Wireless Propagation Letters, vol. 2, no. 14, 2003, pp. $219-222$. 
[118] E. Antonino-Daviu, M. Cabedo-Fabrés, M. Ferrando-Bataller, and A. ValeroNogueira, "Design of Very Wide-band Antennas," JINA 2004 : 13th international symposium on antennas, Nice, France, Nov. 2004.

[119] S. Honda, M. Ito, H. Seki, and Y. Jingo, "A Disc Monopole Antenna with 1:8 Impedance Bandwidth and Omnidirectional Radiation Pattern," Proc. Int. Symp. Antennas Propagat., Sapporo, pp.1145-1148, Japan, Sept.1992.

[120] M. J. Ammann, "Impedance Bandwidth of the Square Planar Monopole", Microwave and Optical Tech. Letters, vol. 24, no. 3, February 2000.

[121] J. A. Evans, and M. J. Ammann, "Planar Trapezoidal and Pentagonal Monopoles with Impedance Bandwidths in Excess of 10:1", IEEE Antennas and Propagation Society International Symposium, vol. 3, pp. 1558 - 1561, July 1999.

[122] M. J Ammann, "The Pentagonal Planar Monopole for Digital Mobile Terminals; Bandwidth Considerations and Modelling," 11th International Conference on Antennas and Propagation, Publ. no. 480, vol. 1, pp. 82-85, April 2001.

[123] Z. N. Chen, "Impedance Characteristics of Planar Bow-tie-like Monopole Antennas", Electronics Letters, vol. 36, no. 13, pp. 1100 - 1101, June 2000.

[124] A. J. Kerkhoff, R. L. Rogers, and H. Ling, "Design and Analysis of Planar Monopole Antennas Using a Genetic Algorithm Approach", IEEE Trans. Antennas Propagat., vol. 2 , pp. 1768 - 1771, June 2004.

[125] E. Lee, P.S. Hall, and P. Gardner, "Novel Compact Wideband or Multi-band Planar Monopole Antenna," Antennas Propag. Society International Symposium, 2000, vol. 2, pp.624-627, IEEE 2000.

[126] E. Lee, P. S. Hall, and P. Gardner, "Compact Wideband Planar Monopole Antenna," Electronic Letters, vol. 35, no.25, pp. 2157-2158, Dec. 1999.

[127] M. J. Ammann, "Wideband Antenna for Mobile Wireless Terminals", Microwave and Optical Tech. Letters, vol. 26, no.6, pp. 360-362, Sep. 2000.

[128] E. Antonino-Daviu, M. Cabedo-Fabres, M. Ferrando-Bataller, and A. ValeroNogueira, "Wideband Double-fed Planar Monopole Antennas". Electronics Letters, vol. 39, no. 23, pp. 1635-1636, November 2003.

[129] A. Kerkhoff, R. Rogers, and H. Ling, "The Use of the Genetic Algorithm Approach in the Design of Ultra-Wideband Antennas," IEEE Radio and Wireless Conference, pp. 93-96, 2001.

[130] P. V. Anob, K. P. Ray, and G. Kumar, "Wideband Orthogonal Square Monopole Antennas with Semi-circular Base," IEEE Antennas and Propagat. Society International Symposium, Vol. 3, pp. 294-297, July 2001.

[131] S. Y. Suh, W. L. Stutzman, and W. A. Davis, "A New Ultrawideband Printed Monopole Antenna: The Planar Inverted Cone Antenna (PICA)," IEEE Trans. Antennas and Propagat., vol. 52, no. 5, pp. 1361 - 1364, May 2004.

[132] M. J. Ammann, and Z. N. Chen, "An Asymmetrical Feed Arrangement for Improved Impedance Bandwidth of Planar Monopole Antennas," Microwave and Optical Technology Letters, vol. 40, no. 2, pp. 156-158, January 2004.

[133] M. J. Ammann, "Control of the Impedance Bandwidth of Wideband Planar Monopole Antennas Using a Beveling Technique", Microwave and Opt. Tech. Lett., vol. 30, no. 4, pp. 229-232, August 2001.

[134] M. Cabedo-Fabrés, A. Valero-Nogueira, and M. Ferrando-Bataller, "A Wideband Arrowhead Planar Monopole Antenna for Multi-service Mobile Systems," Microwave and Optical Tech. Letters, vol. 37, no.3, pp. 188-190, March 2003.

[135] K. L. Wong, C. H. Wu, and S. W. Su, "Ultrawide-band Square Planar Metal-plate Monopole Antenna with a Trident-shaped Feeding Strip," IEEE Trans. Antennas Propagat., vol. 53, no. 4, pp. 1262-1269, April 2005. 
[136] S. W. Su, K. L. Wong, and C. L. Tang, "Ultra-wideband Square Planar Monopole Antenna for IEEE 802.16a Operation in the 2-11-GHz Band," Microwave and Optical Tech. Letters, vol. 42, no.6, pp. 463-466, July 2004.

[137] J. H. Winters, "On the Capacity of Radio Communication Systems with Diversity in a Rayleigh Fading Environment," IEEE J. Select. Areas Commun., vol. 5, pp.871-877, May 1987.

[138] C. Waldschmidt, and W. Wiesbeck, "Compact Wide-Band Multimode Antennas for MIMO and Diversity," IEEE Trans. Antennas Propagat., vol. 52, no. 8, pp. 1963-1969, August 2004.

[139] C. K. Ko and R.D. Murch, "Compact Integrated Diversity Antenna for Wireless Communications," IEEE Transactions on Antennas and Propagation, vol. 49, no 6, pp. 954-960 June 2001.

[140] S. Blanch, J. Romeu and I. Corbella, "Exact Representation of Antenna System Diversity Performance from Input Parameter Description," Electronics Letters, vol. 39, no. 9, May 2003, pp. 705-707.

[141] Z. D. Liu, P. S. Hall, and D. Wake, "Dual Frequency Planar Inverted-F Antenna," IEEE Trans. Antennas and Propagat., no.45, pp.1451-1458, October 1997.

[142] C. Rowell, and R. Murch, "A Compact PIFA Suitable for Dual-frequency 900/1800 MHz Operation," IEEE Trans. Antennas Propagat., no. 4, pp. 596-598, April 1998.

[143] W. P. Dou, and Y. W. M. Chia, "Novel Meandered Planar Inverted-F Antennas for Triple Frequency Operation," Microwave and Opt. Technol. Lett., no. 27, pp. 58-60, October 2000.

[144] Z. N. Chen, and M. Y. W. Chia, "A Feeding Scheme for Enhancing the Impedance Bandwidth of a Suspended Plate Antenna," Microwave Opt. Technol. Lett., vol. 38, no. 1, pp. 21-25, May 2003.

[145] E. Antonino-Daviu, C. A. Suarez-Fajardo, M. Cabedo-Fabrés, and M. FerrandoBataller, "Wideband Antenna for Mobile Terminals Based on the Handset PCB Resonance," Microwave Opt. Technol. Lett., vol. 48, no. 7, pp. 1408-1411, April 2006.

[146] S. K. Sharma, L. Shafai and N. Jacob, "Investigation of Wide-band Microstrip Slot Antenna," IEEE Trans. Antennas Propagat., vol. 52, no. 3, March 2004.

[147] F. Yang, X. Zhang, X. Ye and Y. Rahmat-Samii, "Wide-band E-shaped Patch Antennas for Wireless Communications," IEEE Trans. Antennas Propagat., vol. 49, no. 7, July 2004.

[148] Y. Ge, K.P. Esselle, and T.S. Bird, "E-Shaped Patch Antennas for High-Speed Wireless Networks", IEEE Transactions on Antennas and Propagation, vol.52, no 12, pp. 3213-3219, December 2004.

[149] A. W. Glisson, "On the Development of Numerical Techniques for Treating Arbitrarily-shaped Surfaces," Ph. D. Dissertation, University of Mississippi, 1978.

[150] D. R. Wilton, S. S. M. Rao, and A. W. Glisson, "Electromagnetic Scattering by Arbitrary Surfaces," Rome Air Development Center, Griffis AFB, NY, Tech. Rep. RADC-TR-79-325, March 1980.

[151] J. L. Volakis, "Quadrature Rules for Numerical Integration over Triangles and Tetraedra," IEEE Trans. on Antennas and Propagat., vol. 38, no. 3, June 1996.

[152] S. Rao, "Electromagnetic Scattering and Radiation of Arbitrarily Shaped Surfaces by Triangular Patch Modeling," Ph. D. Thesis, Univ. of Mississippi, 1980.

[153] S. Caorsi, D. Moreno, and F. Sidoti, "Theoretical and Numerical Treatment of Surface Integrals Involving the Free-Space Green's Function," IEEE Trans. on Ant. and Propagat., vol. 41, no. 9, pp. 1296-1301, September 1993. 


\section{Appendix 1. Computation of Eigenvectors and Eigenvalues}

\section{A. Definition of eigenvectors and eigenvalues}

An $N \times N$ matrix $A$ is said to have an eigenvector $x$ and corresponding eigenvalue $\lambda$ if

$$
A \cdot x=\lambda x
$$

Obviously, any multiple of an eigenvector $x$ will also be an eigenvector. The zero vector is not considered to be an eigenvector at all.

Evidently, (A1.1) can hold only if

$$
\operatorname{det}|A-\lambda 1|=0
$$

which if expanded out, is an $N^{\text {th }}$ degree polynomial in $\lambda$ whose roots are the eigenvalues. This proves that there are always $N$ (not necessarily distinct) eigenvalues. Equal eigenvalues coming from multiple roots are called degenerate.

\section{B. Different types of matrices and its associated eigenvalues}

A matrix is called symmetric if it is equal to its transpose

$$
A=A^{T} \quad \text { or } \quad a_{i j}=a_{j i}
$$

It is called Hermitian of self-adjoint if it equals the complex-conjugate of its transpose (its Hermitian conjugate denoted by $\dagger$ )

$$
A=A^{\dagger} \text { or } a_{i j}=a_{j i}{ }^{*}
$$

It is termed orthogona/ if its transpose equals its inverse

$$
A^{T} \cdot A=A \cdot A^{T}=1
$$

and unitary if its Hermitian conjugate equals its inverse.

A matrix is called norma/ if it commutes with its Hermitian conjugate

$$
A^{\dagger} \cdot A=A \cdot A^{\dagger}
$$

For real matrices, Hermitian means the same as symmetric and unitary means the same as orthogonal. 
The eigenvalues of a Hermitian matrix are all real. In particular, the eigenvalues of a real symmetric matrix are all real. Contrariwise, the eigenvalues of a real non-symmetric matrix may include real values, but may also include pairs of complex conjugate values. Finally, eigenvalues of a complex matrix that is not Hermitian will in general be complex.

On the other hand, the eigenvectors of a normal matrix with non-degenerate eigenvalues are complete and orthogonal, spanning the $N$-dimensional vector space. For a normal matrix with degenerate eigenvalues, we have the additional freedom of replacing eigenvectors corresponding to degenerate eigenvalues by linear combinations of themselves.

When a matrix is not normal, in general, we can not find any orthogonal set of eigenvectors, nor even any pairs of eigenvectors that are orthogonal. While the $N$ non-orthogonal eigenvectors will "usually" span de $N$-dimensional vector space, they do not always do so. That is, the eigenvectors are not always complete. These matrices that do not have eigenvalue decomposition are said to be defective or non-diagonalizable.

For defective matrices Schur decomposition can be used instead of eigenvalue decomposition. Using Schur decomposition a defective matrix $A$ can be expressed as

$$
A=U \cdot S \cdot U^{T}
$$

where the columns of $U$ provide a basis with much better numerical properties than a set of eigenvectors.

\section{Generalized eigenvalue problems}

In Chapter 2 is was explained that the eigenvectors and eigenvalues of an antenna can be obtained by solving the following eigenvalue problem

$$
[X] \vec{J}_{n}=\lambda_{n}[R] \vec{J}_{n}
$$

This kind of problem is called generalized eigenvalue problem, and it present the general form

$$
A \cdot x=\lambda B \cdot x
$$

Often $A$ and $B$ are symmetric, and $B$ is positive definite. In this case, the generalized eigenvalues of $A$ and $B$ can be computed using the Cholesky factorization . 
Cholesky factorization expresses the symmetric matrix $B$ as the product of a triangular matrix and its transpose

$$
B=L \cdot L^{T}
$$

where $L$ is an upper triangular matrix.

However, not all symmetric matrices can be factored in this way. The matrices that have a Cholesky factorization are said to be positive definite. This implies that all the diagonal elements of $B$ are positive and that the off-diagonal elements are not too big.

If $B$ is non-singular the problem can be solved by reducing it to an equivalent standard eigenvalue problem

$$
\left(B^{-1} \cdot A\right) \cdot x=\lambda x
$$

If $B$ is a singular matrix, the generalized eigenvalue problem can not be directly transformed into a standard eigenvalue problem, so an alternative algorithm called $Q Z$ factorization is necessary. $Q Z$ factorization is also valid for non-symmetric (non-Hermitian) $A$ and $B$ matrices.

In order to solve the generalized eigenvalue problem in (A1.8), it can be reduced to its standard eigenvalue problem

$$
\left(R^{-1} \cdot X\right) \cdot J_{n}=\lambda_{n} J_{n}
$$

This can be done because $R$ and $X$ matrices correspond with the real and imaginary parts of the generalized impedance matrix of the antenna $Z$, which is a symmetric matrix, since it has been obtained from a Galerkin formulation. Therefore, its hermitian parts $R$ and $X$ are real and symmetric matrices.

Moreover, matrix $R$ is positive semi-defined since the power radiated by a current $J_{n}$ on the surface of a conducting body, is $\left\langle\vec{J}_{n}{ }^{*},[R] \overrightarrow{J_{n}}\right\rangle \geq 0$.

However, in practice, due to roundoff errors, $R$ can be a non-definite matrix, with very small negative eigenvalues. In this case, a Singular Value Decomposition (SVD), already described in section 4.5 of Chapter 4, can be used to solve the problem. 


\section{Computation of Characteristic Modes using Matlab.}

The eigenvalues and eigenvectors of any antenna can be obtained in quite a straight forward way, by solving (A1.8) with Matlab's command eig

$$
[J, D]=\operatorname{eig}(X, R)
$$

where $D$ is the canonical form of $\left(B^{-1} A\right)$, that is, a diagonal matrix with the eigenvalues on the main diagonal, and $J$ is a matrix whose columns are the eigenvectors.

Matlab uses LAPACK routines to compute eigenvalues and eigenvectors, so it selects the most suitable decomposition algorithm depending on the mathematical properties of matrices $R$ and $X$.

Note Matlab's command eig returns the eigenvectors and eigenvalues, only if $R$ and $X$ are square matrices. If the dimension of these matrices is $M \times M$, Matlab will return $M$ eigenvectors and $M$ associated eigenvalues.

It must also be highlighted that eigenvectors yield by the Matlab instruction eig are normalized to have Euclidean length, $\operatorname{norm}(v, 2)$, equal no one. Then, eigenvectors provided by Matlab are not normalized to radiate unit power.

If unnormalized current modes are used, the factor $\left\langle J_{n}, R J_{n}\right\rangle$ must be properly introduced in the theory. As a result in Chapter 2, if unnormalized current modes are considered, orthogonality properties of modes transform in

$$
\begin{gathered}
\left\langle\vec{J}_{m}, R\left(\vec{J}_{n}\right)\right\rangle=P_{n} \cdot \delta_{m n} \\
\left\langle\vec{J}_{m}, X\left(\vec{J}_{n}\right)\right\rangle=P_{n} \cdot \lambda_{n} \cdot \delta_{m n} \\
\left\langle\vec{J}_{m}, Z\left(\vec{J}_{n}\right)\right\rangle=P_{n}\left(1+j \lambda_{n}\right) \delta_{m n}
\end{gathered}
$$

Moreover, when working with unnormalized current modes, equations (2.21) and (2.32) in Chapter 2 must be divided by $P_{n}$.

The information presented in this appendix has been extracted from "Numerical Recipes in C: The art of scientific computing" (www.nr.com) and from Matlab's help. 


\section{Appendix 2. The Mixed Potential Integral Equation}

\section{A. Formulation of the problem}

In Chapter 4 it has been explained that the generalized impedance matrix from which characteristic modes are obtained, is computed using MoM in combination with RWG edge elements. In this appendix the general formulation used to compute this generalized impedance matrix is going to be reviewed.

Consider, as shown in Figure A2.1, a perfectly conducting body which is placed in a homogenous medium $(\mu, \varepsilon)$, and whose surface is denoted by $S$ with unit normal vector $\hat{n}$. The body is illuminated by an incident or impressed field $\left(\vec{E}^{i}, \vec{H}^{i}\right)$, defined to be that which would exist in space if the body were not present. This incident field induces a surface current on the conductor, and this current in turn radiates a scattered field $\left(\vec{E}^{s}, \vec{H}^{s}\right)$. The total field exterior to the body is a superposition of the incident field and the scattered field $\vec{E}=\vec{E}^{i}+\vec{E}^{s}, \vec{H}=\vec{H}^{i}+\vec{H}^{s}$. The induced surface current is unknown, but it is related to the surface transverse magnetic field as $\vec{J}=\hat{n} \times \vec{H}$.

The general idea is to combine Maxwell Equations with boundary conditions in order to derive an integral equation, known as Electric Field Integral Equation (EFIE), which relates the incident electric field with the induced surface current. Then, if the integral equation can be solved for this current, all other electromagnetic quantities may be determined from it.

However, sometimes it is preferred to work with a different formulation of the EFIE that is known as Mixed Potential Integral Equation (MPIE).

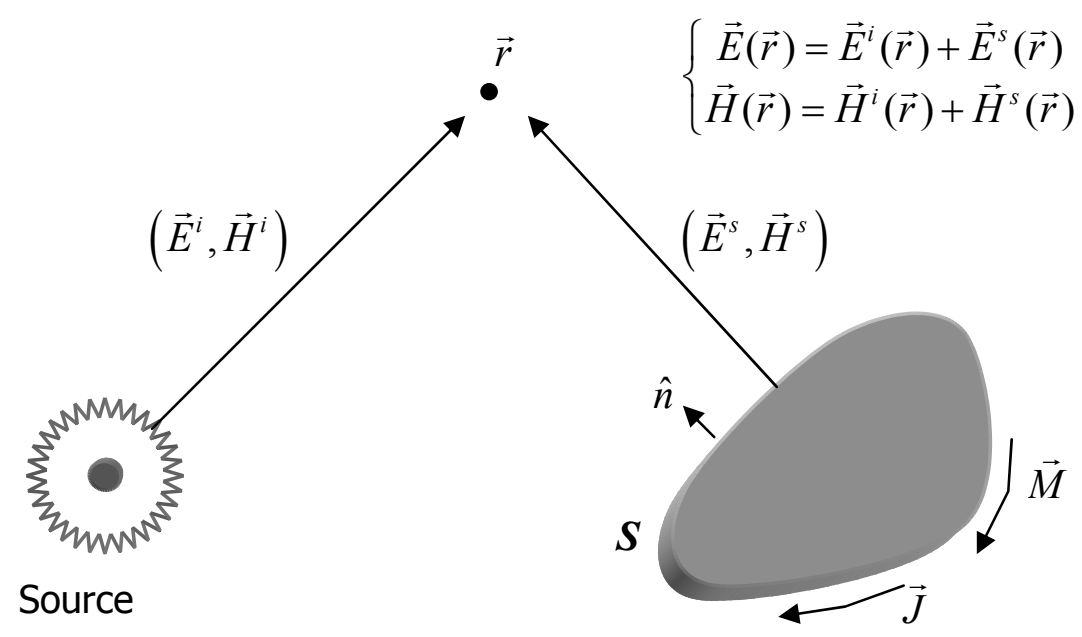

Conducting Object

Figure A2.1. Typical scenario for the problem. 
The MPIE is a particularization of the EFIE, and it relates the surface currents with the vector potential and the gradient of the scalar potential.

The equation for the charge density $\sigma$ and current $J$ on a conducting body $S$ in a known impressed field $\vec{E}^{i}$ is obtained as follows.

As explained in [44], the scattered field $\vec{E}^{s}$ produced by $\sigma$ and $J$, can be expressed in terms of retarded potential integrals as

$$
\vec{E}^{s}(\vec{r})=-j \omega \vec{A}-\nabla \phi
$$

with the magnetic vector potential defined as

$$
\vec{A}(\vec{r})=\frac{\mu}{4 \pi} \iint_{S} \vec{J}_{S}\left(\vec{r}^{\prime}\right) \frac{e^{-j k R}}{R} d S^{\prime}
$$

and the scalar potential as

$$
\phi(\vec{r})=\frac{1}{4 \pi \varepsilon} \int_{S} \sigma \frac{e^{-j k R}}{R} d S^{\prime}
$$

A harmonic time dependence $\exp (j \omega t)$ is assumed and suppressed, and $R=|\vec{r}-\vec{r}|$ is the distance between an arbitrarily located observation point $\vec{r}$ and a source point $\vec{r}^{\prime}$ on $\mathrm{S}$.

The equation of continuity relates the charge density $\sigma$ with the surface divergence of $J$

$$
\nabla_{S} \cdot \vec{J}=-j \omega \sigma
$$

Finally, the boundary condition $\hat{n} \times\left(\vec{E}^{i}+\vec{E}^{s}\right)=0$ on $S$ is applied to obtain

$$
\vec{E}_{\tan }^{i}(\vec{r})=(j \omega \vec{A}+\nabla \phi)_{\tan } \quad \vec{r} \text { on } S
$$

Equation (A2.5) together with (A2.2) and (A2.3) constitutes the MPIE.

But let us see how to use the MPIE in combination with the RWG basis functions and with MoM, with the aim of converting the integral equation into a matrix equation for numerical solution. 


\section{B. RWG basis functions}

In this work, RWG elements have been chosen as basis functions to represent the unknown current on planar structures, since they have been demonstrated to be very suitable for problems involving the EFIE and triangular patch modelling [149].

It is assumed that a suitable triangulation, defined in terms of an appropriate set of faces, edges, vertices, and boundary edges, has been found to approximate $S$. As shown in Figure A2.2 and Figure A2.3, each pair of triangles having a common edge constitutes the corresponding RWG edge element. This means that each basis function is associated to an interior edge of the patch model, and it vanishes everywhere on S except in the two triangles $T_{n}^{+}$and $T_{n}^{-}$ attached to the edge. Points in $T_{n}^{+}$are designated by the position vector $\rho_{n}^{+}$ defined with respect to the free vertex of $T_{n}^{+}$. Similar remarks apply to the position vector $\rho_{n}^{-}$except that it is directed toward the free vertex of $T_{n}^{-}$. The plus or minus design of the triangles is determined by the choice of a positive current reference direction for the $\mathrm{n}^{\text {th }}$ edge, the reference for which is assumed to be from $T_{n}^{+}$to $T_{n}^{-}$. The vector basis function associated with the $\mathrm{n}^{\text {th }}$ edge is defined as

$$
f_{n}(\vec{r})=\left\{\begin{array}{cc}
\frac{l_{n}}{2 A_{n}^{+}} \vec{\rho}_{n}^{+}, \quad \vec{r} \text { in } T_{n}^{+} \\
\frac{l_{n}}{2 A_{n}^{-}} \vec{\rho}_{n}^{-}, \quad \vec{r} \text { in } T_{n}^{-} \\
0, \quad \text { otherwise }
\end{array}\right.
$$

where $l_{n}$ is the length of the edge, and $A_{n}{ }^{ \pm}$is the area of the triangle $T_{n}{ }^{ \pm}$.

More detailed considerations concerning the mathematical representation and topological properties of triangular patch models may be found in [150].

The current on the surface $S$ can be approximated by a sum of the contributions over all the interior edge elements, with unknown coefficients $I_{n}$

$$
\vec{J} \cong \sum_{n=1}^{N} I_{n} \vec{f}_{n}
$$

where $N$ is the number of interior edges.

The unknown coefficients $I_{n}$ can be found from classical moment equations. 


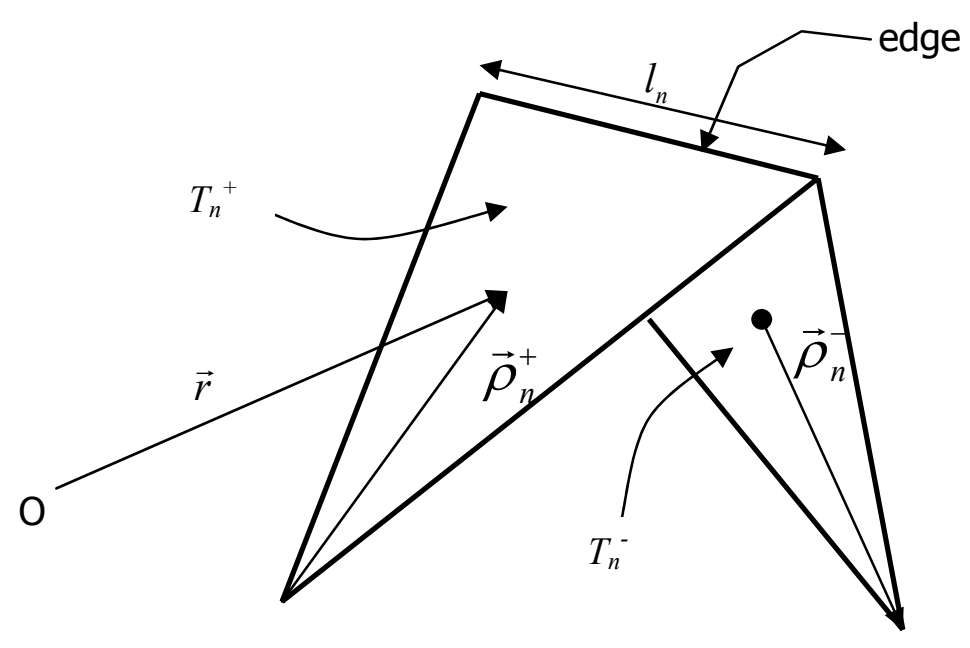

Figure A2.2. Geometrical parameters associated with the $\mathrm{n}^{\text {th }}$ interior edge.

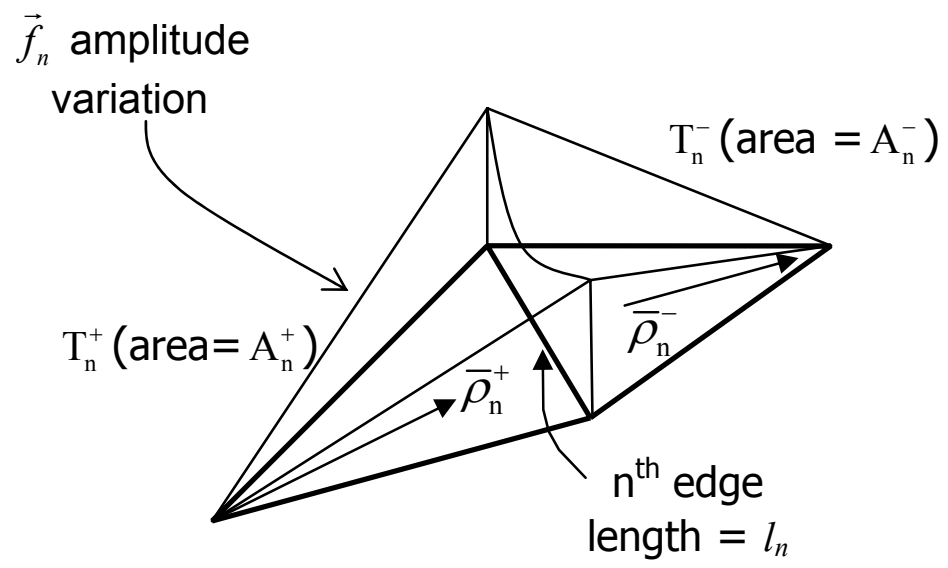

Figure A2.3. RWG basis function description for the $\mathrm{n}^{\text {th }}$ interior edge.

\section{Matrix equation derivation}

Since Galerkin formulation of MoM [42] is going to be applied, RWG basis functions $f_{n}$ are going to be used as expansion and testing functions.

A symmetric product is then defined as

$$
\langle\vec{f}, \vec{g}\rangle \equiv \iint_{S} \vec{f} \cdot \vec{g} d S
$$

Next, for each internal edge, equation A2.5 is tested with the corresponding $f_{m}$ yielding

$$
\left\langle\vec{E}^{i}, \vec{f}_{m}\right\rangle=j \omega\left\langle\vec{A}, \vec{f}_{m}\right\rangle+\left\langle\nabla \phi, \vec{f}_{m}\right\rangle
$$


When the symmetric product of (A2.8) is applied to (A2.9) it results in

$$
\iint_{T_{m}^{+} \cup T_{m}^{-}} \vec{f}_{m} \cdot \vec{E}^{i} d S=\iint_{T_{m}^{+} \cup T_{m}^{-}} j \omega \vec{A} \cdot \vec{f}_{m} d S+\iint_{T_{m}^{+} \cup T_{m}^{-}} \nabla \phi \cdot \vec{f}_{m} d S
$$

Using the distributive property of the $\nabla$ operator, last term in (A2.10) can be rewritten as

$$
\iint_{T_{m}^{+} \cup T_{m}^{-}} \nabla \phi \cdot \vec{f}_{m} d S=\iint_{T_{m}^{+} \cup T_{m}^{-}} \nabla \cdot\left(\phi \vec{f}_{m}\right) d S-\iint_{T_{m}^{+} \cup T_{m}^{-}} \phi \nabla \cdot \vec{f}_{m} d S
$$

By applying Gauss Theorem, and taking into account that the testing functions $f_{m}$ do not have a component normal to the boundary, it can be demonstrated that

$$
\iint_{T_{m}^{+} \cup T_{m}^{-}} \nabla \cdot\left(\phi \vec{f}_{m}\right)=0
$$

Thus, equation (A2.10) can be rewritten as

$$
\iint_{T_{m}^{+} \cup T_{m}^{-}} \vec{f}_{m} \cdot \vec{E}^{i} d S=\iint_{T_{m}^{+} \cup T_{m}^{-}} j \omega \vec{A} \cdot \vec{f}_{m} d S-\iint_{T_{m}^{+} \cup T_{m}^{-}} \phi \nabla \cdot \vec{f}_{m} d S
$$

The magnetic vector potential due to the $\mathrm{n}^{\text {th }}$ interior edge can be expressed as

$$
\vec{A}(\vec{r})=\iint_{S} \vec{J} \frac{e^{-j k R}}{R} d S^{\prime}=\sum_{n=1}^{N} I_{n} \frac{\mu}{4 \pi} \iint_{T_{n}^{+} \cup T_{n}^{-}} \vec{f}_{n}\left(\vec{r}^{\prime}\right) \frac{e^{-j k R}}{R} d S^{\prime}
$$

Similarly, considering (A2.4), the scalar potential can be rewritten as

$$
\begin{aligned}
& \phi(\vec{r})=\frac{1}{4 \pi \varepsilon} \iint_{S} \sigma \frac{e^{-j k R}}{R} d S^{\prime}=\frac{j}{4 \pi \omega \varepsilon} \iint_{S} \nabla^{\prime} \cdot \vec{J} \frac{e^{-j k R}}{R} d S^{\prime} \\
& \phi(\vec{r})=\sum_{n=1}^{N} I_{n}\left[\frac{j}{4 \pi \omega \varepsilon} \iint_{T_{n}^{+} \cup T_{n}^{-}} \nabla^{\prime} \cdot \vec{f}_{n}\left(\vec{r}^{\prime}\right) \frac{e^{-j k R}}{R} d S^{\prime}\right]
\end{aligned}
$$

where $R=|\vec{r}-\vec{r}|$.

Next, $\vec{f}_{n}(\vec{r})$ and $\nabla^{\prime} \vec{f}_{n}\left(\vec{r}^{\prime}\right)$ in (A2.14) and (A2.16) are replaced by its corresponding values giving

$$
\vec{A}_{n}^{ \pm}(\vec{r})=\frac{\mu}{4 \pi} \cdot \frac{l_{n}}{2 A^{q \pm}} \iint_{T^{q \pm}} \vec{\rho}_{n} \frac{e^{-j k\left|\vec{r}-\vec{r}^{\prime}\right|}}{\left|\vec{r}-\vec{r}^{\prime}\right|} d S^{\prime}
$$




$$
\phi_{n}^{ \pm}(\vec{r})= \pm \frac{j l_{n}}{4 \pi \omega \varepsilon} \cdot \frac{1}{A^{q \pm}} \iint_{T^{q \pm}} \frac{e^{-j k\left|\vec{r}-\vec{r}^{\prime}\right|}}{\left|\vec{r}-\vec{r}^{\prime}\right|} d S^{\prime}
$$

Note $\phi_{n}^{ \pm}(\vec{r})$ and $\vec{A}_{n}^{ \pm}(\vec{r})$ are the contributions of the $\mathrm{n}^{\text {th }}$ interior edge associated to source triangles $T^{q+}$ and $T^{q-}$, to the total scalar potential and vector potential, respectively.

Finally, the left part of equation (A2.13) can be transformed in

$$
\begin{aligned}
& \iint_{T_{m}^{+} \cup T_{m}^{+}} j \omega \vec{A} \cdot \vec{f}_{m} d S-\iint_{T_{m}^{+} \cup T_{m}} \phi \nabla \cdot \vec{f}_{m} d S= \\
& =\iint_{T_{m}^{+} \cup T_{m}^{+}} j \omega\left(\sum_{n=1}^{N} I_{n}\left(\vec{A}_{n}^{+}+\vec{A}_{n}^{-}\right)\right) \cdot \vec{f}_{m} d S-\iint_{T_{m}^{+} \cup T_{m}^{+}}\left(\sum_{n=1}^{N} I_{n}\left(\phi_{n}^{+}+\phi_{n}^{-}\right)\right) \nabla \cdot \vec{f}_{m} d S= \\
& =\sum_{n=1}^{N} I_{n}\left[\begin{array}{l}
j \omega l_{m}\left(\frac{1}{2 A_{m}^{+}} \iiint_{T_{m}^{+}}\left(\vec{A}_{n}^{+}+\vec{A}_{n}^{-}\right) \cdot \vec{\rho}_{m}^{+} d S+\frac{1}{2 A_{m}^{-}} \iiint_{T_{m}^{+}}\left(\vec{A}_{n}^{+}+\vec{A}_{n}^{-}\right) \cdot \vec{\rho}_{m}^{-} d S\right) \\
-l_{m}\left(\frac{1}{A_{m}^{+}} \iiint_{T_{m}^{+}}\left(\phi_{n}^{+}+\phi_{n}^{-}\right) d S-\frac{1}{A_{m}^{-}} \iint_{T_{m}^{-}}\left(\phi_{n}^{+}+\phi_{n}^{-}\right) d S\right.
\end{array}\right]
\end{aligned}
$$

and the right part of equation (A2.13) transforms in

$$
\iint_{T_{m}^{+} \cup T_{m}^{-}} \vec{f}_{m} \cdot \vec{E}^{i} d S=l_{m}\left[\frac{1}{2 A_{m}^{+}} \iint_{T_{m}^{+}} \vec{E}^{i} \cdot \vec{\rho}_{m}^{+} d S+\frac{1}{2 A_{m}^{-}} \iint_{T_{m}^{+}} \vec{E}^{i} \cdot \vec{\rho}_{m}^{-} d S\right]
$$

The current density over a perfectly conducting body can be obtained by solving the following system

$$
[Z][I]=[V]
$$

The column vector $[I]$ in (A2.21) is the current density vector, and its elements are the coefficients $I_{n}$ of (A2.7).

The column vector $[V]$ in (A2.21) is called the excitation vector, and its elements are defined as

$$
V_{m}=l_{m}\left[\frac{1}{2 A_{m}^{+}} \iint_{T_{m}^{+}} \vec{E}^{i} \cdot \vec{\rho}_{m}^{+} d S+\frac{1}{2 A_{m}^{-}} \iint_{T_{m}^{+}} \vec{E}^{i} \cdot \vec{\rho}_{m}^{-} d S\right] \quad m=1, \ldots, N
$$


Matriz [ $Z$ ] is an $N \times N$ matrix known as generalized impedance matrix, and its elements $Z_{m n}$ model the coupling between the currents associated to interior edges $m$ and $n$ of the meshing surface

$$
\begin{aligned}
& Z_{m n}=j \omega l_{m}\left(\frac{1}{2 A_{m}^{+}} \iint_{T_{m}^{+}}\left(\vec{A}_{n}^{+}+\vec{A}_{n}^{-}\right) \cdot \vec{\rho}_{m}^{+} d S+\frac{1}{2 A_{m}^{-}} \iint_{T_{m}^{-}}\left(\vec{A}_{n}^{+}+\vec{A}_{n}^{-}\right) \cdot \vec{\rho}_{m}^{-} d S\right) \\
& -l_{m}\left(\frac{1}{A_{m}^{+}} \iint_{T_{m}^{+}}\left(\phi_{n}^{+}+\phi_{n}^{-}\right) d S-\frac{1}{A_{m}^{-}} \iint_{T_{m}^{-}}\left(\phi_{n}^{+}+\phi_{n}^{-}\right) d S\right) \quad m, n=1, \ldots, N
\end{aligned}
$$

where $\vec{A}_{n}^{ \pm}$and $\phi_{n}^{ \pm}$have already been defined in (A2.17) and (A2.18), respectively.

Evidently, $[Z]$ is a square matrix because of the use of Galerkin formulation. Moreover, on the base of the reciprocity theorem $[Z]$ is symmetric. This symmetry of the impedance matrix makes its computation easier.

\section{Efficient numerical evaluation of matrix elements}

To obtain the elements of matrix $[Z], \phi_{n}^{ \pm}(\vec{r})$ and $\vec{A}_{n}^{ \pm}(\vec{r})$ are going to be evaluated at the $\mathrm{m}^{\text {th }}$ edge associated to the basis function $f_{m}$, with $T^{q+}$ and $T^{q-}$ being the triangles attached to the $\mathrm{m}^{\text {th }}$ internal edge. This way, equations (A2.17) and (A2.18) can be rewritten as

$$
\begin{aligned}
& \vec{A}_{n}^{ \pm}\left(\vec{r}_{j}^{p}\right)=\frac{\mu}{4 \pi} \cdot \frac{l_{n}}{2 A^{q \pm}} \iint_{T^{q \pm}} \vec{\rho}_{n} \frac{e^{-j k\left|\vec{r}_{j}^{p}-\vec{r}^{\prime}\right|}}{\left|\vec{r}_{j}^{p}-\vec{r}^{\prime}\right|} d S^{\prime} \\
& \phi_{n}^{ \pm}\left(\vec{r}_{j}^{p}\right)= \pm \frac{j l_{n}}{4 \pi \omega \varepsilon} \cdot \frac{1}{A^{q \pm}} \iint_{T^{q \pm}} \frac{e^{-j k\left|\vec{r}_{j}^{p}-\vec{r}^{\prime}\right|}}{\left|\vec{r}_{j}^{p}-\vec{r}^{\prime}\right|} d S^{\prime}
\end{aligned}
$$

Integrals (A2.24) and (A2.25) are most conveniently evaluated by transforming from the global coordinate system to a local system of coordinates defined within $T^{q}$. To define these coordinates, note that the vectors $\rho_{i}$ in Figure A2.4 divide $T^{q}$ into three subtriangles of areas $A_{1}, A_{2}$, and $A_{3}$, with $l_{1}, l_{2}$ and $l_{3}$, respectively, as one of their sides. The areas are not independent, however, they must satisfy $A_{1}+A_{2}+A_{3}=A^{q}$. 


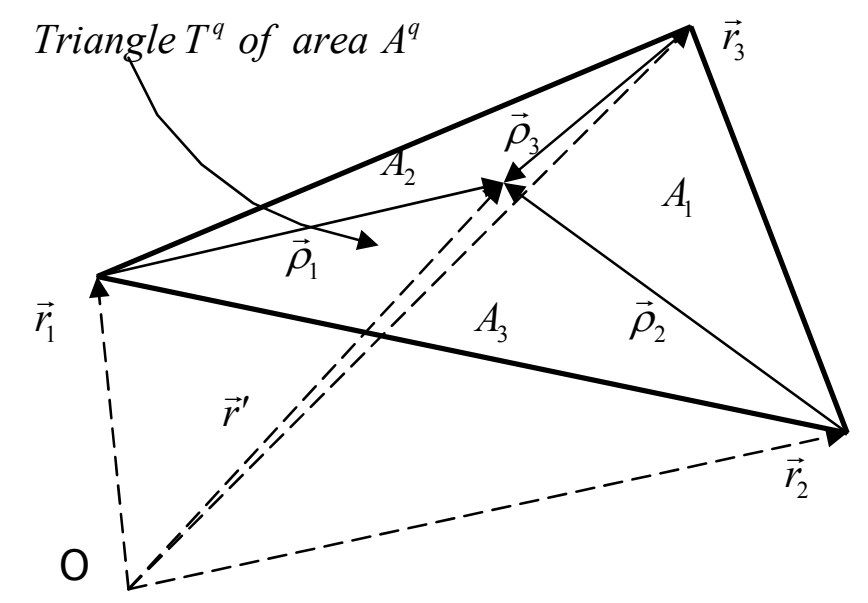

Figure A2.4. Local coordinates and axis for the source triangle $T^{q}$

Now, the normalized area coordinates can be defined as

$$
\begin{aligned}
\xi & =\frac{A_{1}}{A^{q}} \\
\eta & =\frac{A_{2}}{A^{q}} \\
\tau & =\frac{A_{3}}{A^{q}}
\end{aligned}
$$

Because of the area constraint these coordinates must satisfy

$$
\xi+\eta+\tau=1
$$

Note that all three coordinates vary between zero and unity in $T^{q}$ and that at the triangle corners $\vec{r}_{1}, \vec{r}_{2}$ y $\vec{r}_{3}$, the triplet $(\xi, \eta, \tau)$ takes on the values $(1,0,0)$, $(0,1,0)$ and $(0,0,1)$, respectively. The transformation from Cartesian to normalized area coordinates may be written in vector form as

$$
\begin{gathered}
\vec{r}^{\prime}=\xi \vec{r}_{1}+\eta \vec{r}_{2}+\tau \vec{r}_{3} \\
\vec{r}^{\prime}=\xi \vec{r}_{1}+\eta \vec{r}_{2}+(1-\xi-\eta) \vec{r}_{3} \\
\vec{\rho}_{n}^{ \pm}= \pm\left(\vec{r}^{\prime}-\vec{r}_{n}\right)= \pm\left[\left(\vec{r}_{1}-\vec{r}_{3}\right) \xi+\left(\vec{r}_{2}-\vec{r}_{3}\right) \eta+\left(\vec{r}_{3}-\vec{r}_{n}\right)\right] \quad n=1,2,3 \text { vertex }
\end{gathered}
$$


It can easily be demonstrated that surface integrals over $T^{q}$ transform as follow

$$
\iint_{T^{q}} g(\vec{r}) d S=2 A^{q} \int_{0}^{1} \int_{0}^{1-\eta} g\left(\xi \vec{r}_{1}+\eta \vec{r}_{2}+(1-\xi-\eta) \vec{r}_{3}\right) d \xi d \eta
$$

By applying (A2.33) and substituting (A2.32) in (A2.24), $\vec{A}_{n}^{ \pm}\left(\vec{r}_{j}^{p}\right)$ can be expressed as

$$
\vec{A}_{n}^{ \pm}\left(\vec{r}_{j}^{p}\right)= \pm \frac{\mu}{4 \pi} \frac{l_{n}}{2 A^{q \pm}} 2 A^{q \pm} \int_{0}^{1} \int_{0}^{1-\eta^{q}}\left[\left(\vec{r}_{1}^{q}-\vec{r}_{3}^{q}\right) \xi^{q}+\left(\vec{r}_{2}^{q}-\vec{r}_{3}^{q}\right) \eta^{q}+\left(\vec{r}_{3}^{q}-\vec{r}_{n}^{q}\right)\right] \frac{e^{-j k R_{j}^{p q}}}{R_{j}^{p q}} d \xi^{q} d \eta^{q}
$$

where $R_{j}^{p q}=\left|\vec{r}_{j}^{p}-\vec{r}^{q}\right|$.

The physical meaning of $R_{j}^{p q}$ is the distance between an observation point $j$ located at triangle $p$, and a source point located at triangle $q$.

A more compact expression for $\vec{A}_{n}^{ \pm}\left(\vec{r}_{j}^{p}\right)$ is

$$
\vec{A}_{n}^{ \pm}\left(\vec{r}_{j}^{p}\right)= \pm \frac{\mu l_{n}}{4 \pi}\left[\left(\vec{r}_{1}^{q}-\vec{r}_{3}^{q}\right) I_{\xi_{q}}^{p q}+\left(\vec{r}_{2}^{q}-\vec{r}_{3}^{q}\right) I_{\eta_{q}}^{p q}+\left(\vec{r}_{3}^{q}-\vec{r}_{n}^{q}\right) I^{p q}\right]
$$

with

$$
\begin{gathered}
I^{p q}\left(\vec{r}_{j}^{p}\right)=\int_{0}^{1} \int_{0}^{1-\eta^{q}} \frac{e^{-j k R_{j}^{p q}}}{R_{j}^{p q}} d \xi^{q} d \eta^{q} \\
I_{\xi}^{p q}\left(\vec{r}_{j}^{p}\right)=\int_{0}^{1} \int_{0}^{1-\eta^{q}} \xi^{q} \frac{e^{-j k R_{j}^{p q}}}{R_{j}^{p q}} d \xi^{q} d \eta^{q} \\
I_{\eta}^{p q}\left(\vec{r}_{j}^{p}\right)=\int_{0}^{1} \int_{0}^{1-\eta^{q}} \eta^{q} \frac{e^{-j k R_{j}^{p q}}}{R_{j}^{p q}} d \xi^{q} d \eta^{q}
\end{gathered}
$$

Likewise, an alternative expression can be derived for $\phi_{n}^{ \pm}\left(\vec{r}_{j}^{p}\right)$

$$
\phi_{n}^{ \pm}\left(\vec{r}_{j}^{p}\right)= \pm \frac{j l_{n}}{2 \pi \omega \varepsilon} I^{p q}\left(\vec{r}_{j}^{p}\right)
$$


Thus, only three independent integrals must be evaluated for each combination of triangles $p$ and $q$. Then, the elements $Z_{m n}$ of matrix [Z] can be expressed as a linear combination of integrals that are independent of the observation and source edges. Each triangle presents a maximum of three internal edges, so the advantage of using the previously described formulation is obvious, since all the contributions coming from the edges of triangles $\left(T^{q}, T^{p}\right)$ to the impedance matrix, can be derived just from the three integrals $I^{p q}, I_{\xi}^{p q}$ and $I_{\eta}^{p q}$. As a result, the total number of matrix elements requiring evaluation of the same potential integrals can be as large as nine.

When the source triangle and the observation triangle are far away from each other, integrals $I^{p q}, I_{\xi}^{p q}$ and $I_{\eta}^{p q}$, present a soft variation over the surface of $T^{q}$. In this case, the abovementioned integrals can be evaluated numerically. The numerical evaluation is based on the use of numerical quadrature techniques [151] specially developed for triangular domains

$$
I^{p q}\left(\vec{r}_{j}^{p}\right)=\int_{0}^{1} \int_{0}^{1-\eta^{q}} \frac{e^{-j k R_{j}^{p q}}}{R_{j}^{p q}} d \xi^{q} d \eta^{q}=\sum_{i=1}^{N_{i}} \alpha_{i} \frac{e^{-j k R_{j i}^{p q}}}{R_{j i}^{p q}}
$$

where:

$N_{i}$ : Number of points evaluated over the source triangle.

$\alpha_{i}$ : Weighting coefficient.

$R_{j i}^{p q}$ : Distance between an observation point located at triangle $p$, and a source point located at triangle $q$.

$$
R_{j i}^{p q}=\left|\vec{r}_{j}^{p}-\vec{r}_{i}^{q}\right|=\left|\left(\left(\vec{r}_{1}^{p}-\vec{r}_{3}^{p}\right) \xi_{j}^{p}+\left(\vec{r}_{2}^{p}-\vec{r}_{3}^{p}\right) \eta_{j}^{p}+\vec{r}_{3}^{p}\right)-\left(\left(\vec{r}_{1}^{q}-\vec{r}_{3}^{q}\right) \xi_{i}^{q}+\left(\vec{r}_{2}^{q}-\vec{r}_{3}^{q}\right) \eta_{i}^{q}+\vec{r}_{3}^{q}\right)\right|
$$

$\left(\xi_{i}^{q}, \eta_{i}^{q}\right)$ are the coordinates for an integration point $i$ over source triangle $q$. $\left(\xi_{j}^{p}, \eta_{j}^{p}\right)$ are the coordinates for an integration point $j$ over source triangle $p$.

However, for the terms in which $p=q$ the integrands are singular and for these cases, the singular portion of each integrand must be removed and integrated analytically [152]-[153]. 


\section{$\underline{\text { Related Publications }}$}

\section{International Magazines}

[1] M. Cabedo-Fabrés, A. Valero-Nogueira, and M. Ferrando-Bataller, "A Wideband Arrowhead Planar Monopole Antenna for Multi-Service Mobile Systems," Microwave and Optical Tech. Letters, vol. 37, no.3, pp. 188-190, March 2003.

[2] E. Antonino, M. Cabedo, M. Ferrando, A. Valero, "Novel Wide-band Double-fed Planar Monopole Antennas," Electronics Letters, vol. 39, November 2003, pp.16351636.

[3] E. Antonino-Daviu, C. A. Suarez-Fajardo , M. Cabedo-Fabrés, and M. FerrandoBataller, "Wideband Antenna for Mobile Terminals Based on the Handset PCB Resonance," Microwave Opt. Technol. Lett., vol. 48, no. 7, pp. 1408-1411, April 2006.

[4] M. Cabedo-Fabrés, E. Antonino-Daviu, A. Valero-Nogueira, and M. FerrandoBataller, "The Theory of Characteristic Modes Revisited: A Contribution to the Design of Antennas for Modern Applications," Accepted for publication in IEEE Trans. Antennas Propagat. Magazine. Expected publication date: October 2007.

[5] M. Cabedo-Fabrés, E. Antonino-Daviu, A. Valero-Nogueira, and M. FerrandoBataller, "Notched Radiating Ground Plane Analyzed from a Modal Perspective," Accepted for publication in Frequenz. Expected publication date: March 2007.

[6] N. Belmar-Moliner, A. Valero-Nogueira, M. Cabedo-Fabrés and E. Antonino-Daviu, "Simple design for cost-effective diversity antennas" Microwave Opt. Technol. Lett., vol. 49, no. 4, pp. 994-996, April 2007.

\section{International Conferences}

[1] M. Cabedo-Fabrés, M. Ferrando-Bataller, and A. Valero-Nogueira, "Systematic study of elliptical loop antennas using Characteristic Modes," Antennas and Propagation Society International Symposium, 2002. IEEE

Vol. 1, 16-21 June 2002, Page(s):156 - 159.

[2] M. Cabedo, M. Ferrando and A. Valero, "Innovative wide-band planar monopole antenna for multi-service mobile systems", Journées Internationales de Nice sur les Antennes - International Symposium on Antennas, Nice, November 2002.

[3] M. Cabedo-Fabrés, E. Antonino-Daviu, M. Ferrando-Bataller and A. ValeroNogueira, "On the use of Characteristic Modes to describe patch antenna performance," Antennas and Propagation Society International Symposium, 2003. IEEE Vol. 2, 22-27 June 2003, Page(s):712 - 715.

[4] M. Cabedo-Fabrés, E. Antonino-Daviu, A. Valero-Nogueira and M. FerrandoBataller, "Analysis of wide band planar monopole antennas using Characteristic Modes," Antennas and Propagation Society International Symposium, 2003. IEEE Vol. 3, 22-27 June 2003, Page(s):733 - 736.

[5] M. Ferrando-Bataller, A. Valero-Nogueira, M. Cabedo-Fabrés and E. AntoninoDaviu, "Design of ultra-wideband antennas using Characteristic Modes," INICA/COST 284 Workshop, Berlin, Germany, 2003. 
[6] M. Cabedo-Fabrés, E. Antonino-Daviu, A. Valero-Nogueira, and M. FerrandoBataller, "Controlled antenna design method based on the use of Characteristic Modes," PIERS 2004, Pisa, Italy, March 2004.

[7] E. Antonino-Daviu, M. Cabedo-Fabrés, M. Ferrando-Bataller, and J. I. Herranz, "Analysis of the coupled chassis-antenna modes in mobile handsets," Antennas and Propagation Society International Symposium, 2004. IEEE Vol. 3, 20-25 June 2004, Page(s):2751 - 2754.

[8] E. Antonino-Daviu, M. Cabedo-Fabrés, M. Ferrando-Bataller, and A. ValeroNogueira, "A discussion on the feed configuration of planar monopole antennas to obtain ultra wideband performance," Antennas and Propagation Society International Symposium, 2004. IEEE Vol. 2, 20-25 June 2004, Page(s):1867 1870.

[9] M. Cabedo-Fabrés, E. Antonino-Daviu, A. Valero-Nogueira, and M. FerrandoBataller, "Optimization of the polarization of reflectarrays using Characteristic Modes," Antennas and Propagation Society International Symposium, 2004. IEEE Vol. 1, 20-25 June 2004, Page(s):13 - 16.

[10] M. Cabedo-Fabrés, A. Valero-Nogueira, J.I. Herranz-Herruzo and M. FerrandoBataller, "A discussion on the Characteristic Mode Theory limitations and its improvement for the effective modelling of antennas and arrays," Antennas and Propagation Society International Symposium, 2004. IEEE Vol.1, 20-25 June 2004, Page(s):121 - 124.

[11] M. Cabedo-Fabrés, E. Antonino-Daviu, A. Valero-Nogueira, and M. FerrandoBataller, "Systematic antenna design using the Theory of Characteristic Modes," JINA 2004, International Symposium on Antennas, Nice, France, November 2004.

[12] E. Antonino-Daviu, M. Cabedo-Fabrés, A. Valero-Nogueira and M. FerrandoBataller, "Design of very wide-band linear polarized antennas," JINA 2004, International Symposium on Antennas, Nice, France, November 2004.

[13] E. Antonino-Daviu, M. Cabedo-Fabrés, A. Valero-Nogueira and M. FerrandoBataller, "Resonant modes in antenna handsets," INICA/COST 284 Workshop, Gothenburg, Sweeden, 2004.

[14] E. Antonino-Daviu, M. Cabedo-Fabrés, M. Ferrando-Bataller, A. Valero-Nogueira and Marta Martínez-Vazquez, "Novel antenna for mobile terminals based on the chassis-antenna coupling," Antennas and Propagation Society International Symposium, 2005. IEEE, July 2005.

[15] M. Cabedo-Fabrés, E. Antonino-Daviu, A. Valero-Nogueira, and M. FerrandoBataller, "Wideband Radiating Ground Plane with Notches," Antennas and Propagation Society International Symposium, 2005. IEEE, July 2005.

[16] M. Cabedo-Fabrés, A. Valero-Nogueira, E. Antonino-Daviu, and M. FerrandoBataller, "Modal analysis of a radiating slotted PCB for mobile handsets," European Conference on Antenna and Propagation (EUCAP), Nov. 2006.

[17] M. Ferrando-Bataller, M. Cabedo-Fabrés, E. Antonino-Daviu, and A. ValeroNogueira, "Overview of planar monopole antenas for UWB applications," European Conference on Antenna and Propagation (EUCAP), Nov. 2006. 


\section{National Conferences}

[1] M. Cabedo-Fabrés, M. Ferrando-Bataller y A. Valero-Nogueira, "Nuevo monopolo plano de banda ancha para comunicaciones móviles multiservicio," XVII Simposium Nacional de la Unión Científica Internacional de Radio URSI 2002, Alcalá de Henares.

[2] M. Cabedo-Fabrés, A. Valero-Nogueira y M. Ferrando-Bataller, "Estudio sistemático de antenas de hilo empleando modos característicos," XVII Simposium Nacional de la Unión Científica Internacional de Radio URSI 2002, Alcalá de Henares.

[3] M. Cabedo-Fabrés, A. Valero-Nogueira, M. Ferrando-Bataller, y J. I. HerranzHerruzo, "Aplicación de la teoría de modos característicos al modelado efectivo de antenas y arrays," XVIII Simposium Nacional de la Unión Científica Internacional de Radio URSI 2003, La Coruña.

[4] M. Cabedo-Fabrés, E. Antonino-Daviu, M. Ferrando-Bataller, y A. Valero-Nogueira, "Modos característicos de estructuras planas de forma arbitraria," XVIII Simposium Nacional de la Unión Científica Internacional de Radio URSI 2003, La Coruña.

[5] E. Antonino-Daviu, M. Cabedo-Fabrés, M. Ferrando-Bataller, y A. Valero-Nogueira "Diseño de monopolos planos con alimentación múltiple a partir de la teoría de los modos característicos," XVIII Simposium Nacional de la Unión Científica Internacional de Radio URSI 2003, La Coruña.

[6] E. Antonino-Daviu, M. Cabedo-Fabrés, M. Ferrando-Bataller, y A. Valero-Nogueira "Nueva antena de banda ancha y polarización dual," XVIII Simposium Nacional de la Unión Científica Internacional de Radio URSI 2003, La Coruña.

[7] M. Cabedo-Fabrés, E. Antonino-Daviu, A. Valero-Nogueira, y M. Ferrando-Bataller, "Diseño de antenas de hendidura en plano de masa finito," XIX Simposium Nacional de la Unión Científica Internacional de Radio URSI 2004, Barcelona.

[8] M. Cabedo-Fabrés, E. Antonino-Daviu, A. Valero-Nogueira, y M. Ferrando-Bataller, "Estudio modal de resonadores abiertos acoplados," XIX Simposium Nacional de la Unión Científica Internacional de Radio URSI 2004, Barcelona.

[9] E. Antonino-Daviu, M. Cabedo-Fabrés, M. Ferrando-Bataller, y A. Valero-Nogueira "Efecto de las discontinuidades en la radiación de estructuras planas," XIX Simposium Nacional de la Unión Científica Internacional de Radio URSI 2004, Barcelona.

[10] E. Antonino-Daviu, M. Cabedo-Fabrés, M. Ferrando-Bataller, y A. Valero-Nogueira "Acoplamiento de modos resonantes en terminales móviles," XIX Simposium Nacional de la Unión Científica Internacional de Radio URSI 2004, Barcelona.

[11] M. Cabedo-Fabrés, E. Antonino-Daviu, D. Sánchez-Escuderos, M. FerrandoBataller, "On the Influence of the Shape of Planar Monopole Antennas in the Impedance Bandwidth Performance," Sesión especial de ACE durante el XX Simposium Nacional de la Unión Científica Internacional de Radio URSI 2005, Gandía.

[12] E. Antonino-Daviu, M. Cabedo Fabres, F. Vico Bondia, C. Suarez Fajardo, "Nueva antena multibanda para terminales móviles basada en la generación de resonancias en el plano de masa del Terminal," XX Simposium Nacional de la Unión Científica Internacional de Radio URSI 2005, Gandía.

[13] A. Valero-Nogueira, M. Cabedo-Fabrés, E. Antonino-Daviu, "Diseño de una antena compacta para sistemas MIMO," XX Simposium Nacional de la Unión Científica Internacional de Radio URSI 2005, Gandía.

[14] M. Cabedo-Fabrés, E. Antonino-Daviu, y V. M. Rodrigo Peñarrocha, "Análisis modal de un plano de masa radiante doblado y con una ranura para terminales móviles," XXI Simp. Nacional de la Unión Científica Int. de Radio URSI 2006, Oviedo. 
